\title{
Unexpected Insertion of Nitrogen into a C-C Bond: Access to 2,3-Disubstituted Quinazolinone Scaffolds
}

Hui-Li Liu, Xiao-Tong Li, Heng-Zhi Tian, Xing-Wen Sun*

*Department of Chemistry, Fudan University, Shanghai 200433, China

E-mail: sunxingwen@fudan.edu.cn

\section{Table of Contents}

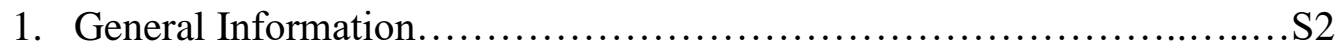

2. Characterization Data of the Known Start Materials.....................S2

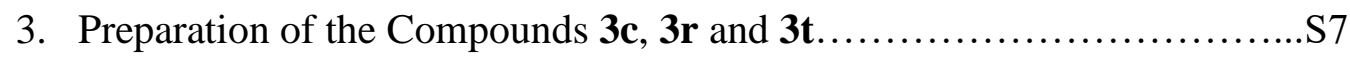

4. General Procedure for the synthesis of 5aa-5sa, 5ab-5az, 6ta............S11

5. Gram-Scale Synthesis of 5ar..................................... 26

6. Procedure for the Synthesis of 8ar and 9ar.......................S26

7. The Detected and Separated of the Intermediates...................................S28

8. Variation of the EWG at 2-position in 3-indolinone Scaffold....................S29

9. The Other Type Nitroso-Compound were Examined..................................S30

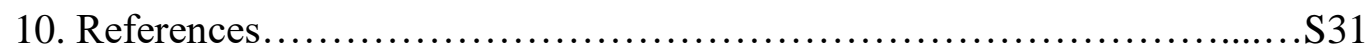

11. X-ray Data of the Products....................................... 33

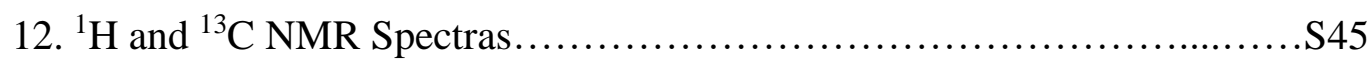




\section{General Information}

All of the reagents and solvents were purchased from commercial sources, and were used without further purification unless specified. Melting points were recorded with a micro melting point apparatus and are uncorrected. ${ }^{1} \mathrm{H}$ NMR and ${ }^{13} \mathrm{C}$ NMR spectra were recorded on commercial instruments Bruker Avance III HD (400 MHz). Chemical shifts are recorded in ppm relative to tetramethylsilane and with the solvent resonance as the internal standard. Data are reported as follows: chemical shift, multiplicity ( $\mathrm{s}=$ singlet, $\mathrm{d}=$ doublet, $\mathrm{t}=$ triplet, $\mathrm{q}=$ quartet, $\mathrm{dd}=$ doublet of doublets, $\mathrm{m}=$ multiplet) coupling constants $(\mathrm{Hz})$, integration. Chemical shifts are reported in ppm from the tetramethylsilane with the solvent resonance as internal standard. ESI were recorded on a Bruker ApeXIII 7.0 TESLA FTMS Mass Spectrum. The X-ray crystallographic analysis were obtained on a Bruker D8 Venture diffractometer. All experiments were monitored by analytical thin layer chromatography (TLC). All reactions were carried out in a sealed tube. The crude reaction mixture was purified by flash column chromatography on silica gel (300-400 mesh) and afforded the target product.

\section{Characterization Data of the Known Start Materials}

2.1 The compounds $\mathbf{3 a - 3 b}, \mathbf{3 d - 3 q}, \mathbf{3 s}$ were synthesized according to the known literature procedures. ${ }^{[1]}$
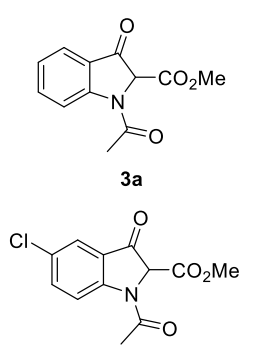

$3 \mathrm{~g}$

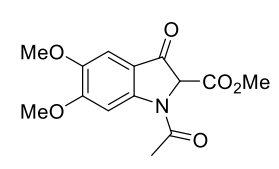

3 I

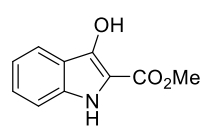

$3 q$

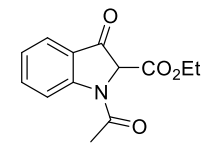

3b

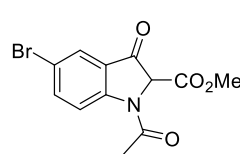

$3 \mathbf{h}$

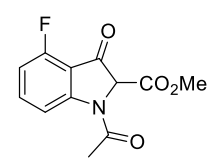

$3 \mathrm{~m}$

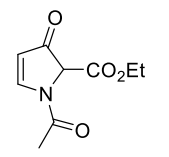

$3 s$

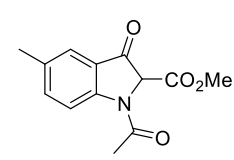

3d

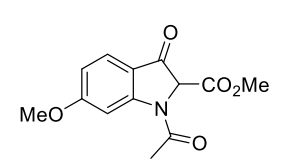

$3 i$

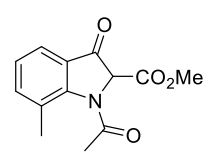

$3 n$

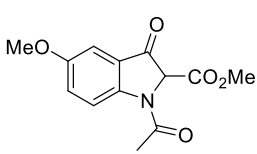

$3 e$

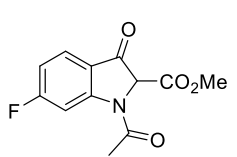

3j

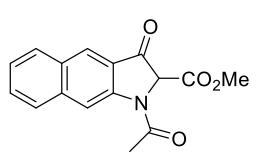

30
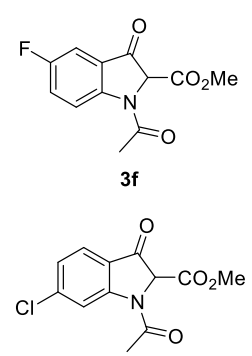

$3 k$

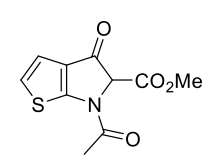

$3 p$ 

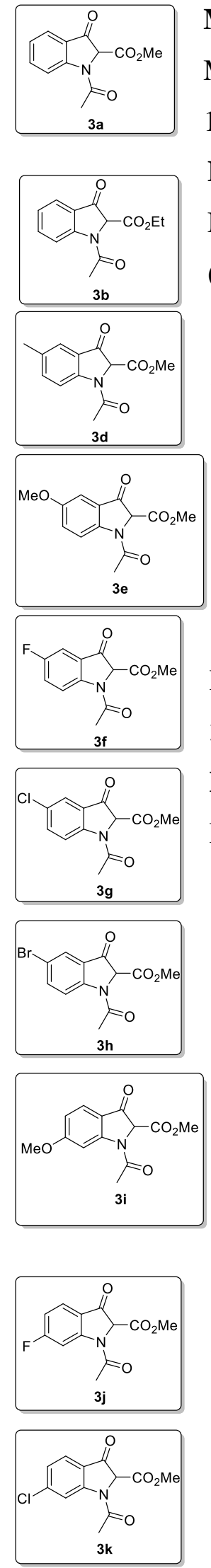

Methyl 1-acetyl-3-oxoindoline-2-carboxylate (3a). ${ }^{1} \mathrm{H}$ NMR (400 $\left.\mathrm{MHz}, \mathrm{CDCl}_{3}\right) \delta$ 8.76-7.40 (m, 3H), 7.25-7.13 (m, 1H), 5.18-4.86 (m, $1 \mathrm{H}), 3.86(\mathrm{~s}, 3 \mathrm{H}), 2.75-2.00(\mathrm{~m}, 3 \mathrm{H})$.

Ethyl 1-acetyl-3-oxoindoline-2-carboxylate (3b). ${ }^{1} \mathrm{H}$ NMR (400 $\left.\mathrm{MHz}, \mathrm{CDCl}_{3}\right) \delta 8.60-7.40(\mathrm{~m}, 3 \mathrm{H}), 7.17$ (t, $\left.J=7.6 \mathrm{~Hz}, 1 \mathrm{H}\right), 5.08-4.90$ (s, 1H), 4.34-4.19 (m, 2H), 2.75-2.05 (m, 3H), 1.27 (t, $J=7.2 \mathrm{~Hz}, 3 \mathrm{H})$. Methyl 1-acetyl-5-methyl-3-oxoindoline-2-carboxylate (3d). ${ }^{1} \mathrm{H}$ $\operatorname{NMR}\left(400 \mathrm{MHz}, \mathrm{CDCl}_{3}\right) \delta 8.50-7.34(\mathrm{~m}, 3 \mathrm{H}), 5.12-4.88(\mathrm{~m}, 1 \mathrm{H}), 3.82$ $(\mathrm{s}, 3 \mathrm{H}), 2.34(\mathrm{~s}, 3 \mathrm{H}), 2.60-2.15(\mathrm{~m}, 3 \mathrm{H})$.

Methyl 1-acetyl-5-methoxy-3-oxoindoline-2-carboxylate $(3 \mathrm{e}) .{ }^{1} \mathrm{H}$ NMR (400 MHz, $\left.\mathrm{CDCl}_{3}\right) \delta$ 8.52-7.18 (m, 2H), 7.16-6.96 (m, 1H), 5.12-4.90 (m, 1H), $3.81(\mathrm{~s}, 3 \mathrm{H}), 3.77(\mathrm{~s}, 3 \mathrm{H}), 2.55-2.08(\mathrm{~s}, 3 \mathrm{H})$.

Methyl 1-acetyl-5-fluoro-3-oxoindoline-2-carboxylate (3f). ${ }^{1} \mathrm{H}$ NMR (400 MHz, $\left.\mathrm{CDCl}_{3}\right) \delta$ 8.62-7.27 (m, 3H), $5.04(\mathrm{~s}, 1 \mathrm{H}), 3.82(\mathrm{~s}$, $3 \mathrm{H}), 2.70-2.00(\mathrm{~m}, 3 \mathrm{H})$.

Methyl 1-acetyl-5-chloro-3-oxoindoline-2-carboxylate $\quad(3 \mathrm{~g}) .{ }^{1} \mathrm{H}$ NMR (400 MHz, $\left.\mathrm{CDCl}_{3}\right) \delta 8.55(\mathrm{~s}, 1 \mathrm{H}), 7.76-7.49(\mathrm{~m}, 2 \mathrm{H}), 5.03(\mathrm{~s}$, $1 \mathrm{H}), 3.86(\mathrm{~s}, 3 \mathrm{H}), 2.65-2.05(\mathrm{~m}, 3 \mathrm{H})$.

Methyl 1-acetyl-5-bromo-3-oxoindoline-2-carboxylate $(3 \mathrm{~h}) .{ }^{1} \mathrm{H}$ NMR (400 MHz, $\left.\mathrm{CDCl}_{3}\right) \delta 8.49(\mathrm{~s}, 1 \mathrm{H}), 7.90-7.69(\mathrm{~m}, 2 \mathrm{H}), 5.02(\mathrm{~s}$, $1 \mathrm{H}), 3.86(\mathrm{~s}, 3 \mathrm{H}), 2.68-2.09(\mathrm{~m}, 3 \mathrm{H})$.

Methyl 1-acetyl-6-methoxy-3-oxoindoline-2-carboxylate (3i). ${ }^{1} \mathrm{H}$ NMR $\left(400 \mathrm{MHz}, \mathrm{CDCl}_{3}\right) \delta 8.05(\mathrm{~s}, 1 \mathrm{H}), 7.55(\mathrm{~d}, J=8.0 \mathrm{~Hz}, 1 \mathrm{H})$, $6.69(\mathrm{~d}, J=8.4 \mathrm{~Hz}, 1 \mathrm{H}), 4.96(\mathrm{~s}, 1 \mathrm{H}), 3.87(\mathrm{~s}, 3 \mathrm{H}), 3.81(\mathrm{~s}, 3 \mathrm{H})$, 2.58-2.05 (m, 3H).

Methyl 1-acetyl-6-fluoro-3-oxoindoline-2-carboxylate $\quad(3 \mathbf{j}) . \quad{ }^{1} \mathrm{H}$ $\operatorname{NMR}\left(400 \mathrm{MHz}, \mathrm{CDCl}_{3}\right) \delta 8.44-7.47(\mathrm{~m}, 2 \mathrm{H}), 6.88(\mathrm{t}, J=8.4 \mathrm{~Hz}, 1 \mathrm{H})$, $5.03(\mathrm{~s}, 1 \mathrm{H}), 3.81(\mathrm{~s}, 3 \mathrm{H}), 2.61-2.04(\mathrm{~m}, 3 \mathrm{H})$.

Methyl 1-acetyl-6-chloro-3-oxoindoline-2-carboxylate $\quad(3 k) .{ }^{1} \mathrm{H}$ NMR (400 MHz, $\left.\mathrm{CDCl}_{3}\right) \delta 8.64(\mathrm{~s}, 1 \mathrm{H}), 7.65(\mathrm{~d}, J=7.2 \mathrm{~Hz}, 1 \mathrm{H}), 7.20$ $(\mathrm{dd}, J=8.2,1.2 \mathrm{~Hz}, 1 \mathrm{H}), 5.02(\mathrm{~s}, 1 \mathrm{H}), 3.86(\mathrm{~s}, 3 \mathrm{H}), 2.72-2.10(\mathrm{~m}, 3 \mathrm{H})$. 


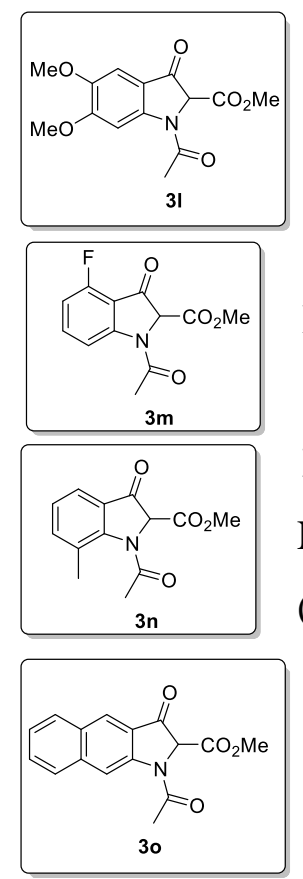

Methyl 1-acetyl-5,6-dimethoxy-3-oxoindoline-2-carboxylate (31).

${ }^{1} \mathrm{H}$ NMR (400 MHz, $\left.\mathrm{CDCl}_{3}\right) \delta 8.18(\mathrm{~s}, 1 \mathrm{H}), 7.07$ (s, 1H), 4.95 (s, $1 \mathrm{H}), 4.01(\mathrm{~s}, 3 \mathrm{H}), 3.87$ (s, 3H), $3.86(\mathrm{~s}, 3 \mathrm{H}), 2.18$ (s, 3H).

Methyl 1-acetyl-4-fluoro-3-oxoindoline-2-carboxylate $(3 \mathrm{~m}) .{ }^{1} \mathrm{H}$ NMR (400 MHz, $\left.\mathrm{CDCl}_{3}\right) \delta 8.50-7.51(\mathrm{~m}, 2 \mathrm{H}), 6.80(\mathrm{t}, J=8.4 \mathrm{~Hz}, 1 \mathrm{H})$, $5.01(\mathrm{~s}, 1 \mathrm{H}), 3.82(\mathrm{~s}, 3 \mathrm{H}), 2.72-1.97(\mathrm{~m}, 3 \mathrm{H})$.

Methyl 1-acetyl-7-methyl-3-oxoindoline-2-carboxylate $(3 n) .{ }^{1} \mathrm{H}$ NMR (400 MHz, $\left.\mathrm{CDCl}_{3}\right) \delta$ 7.62-7.45 (m, 2H), 7.25-7.16 (m, 1H), 5.01 (s, 1H), 3.88-3.76 (m, 3H), 2.41-2.34 (m, 3H), 2.27-2.19 (m, 3H).

\section{Methyl \\ 1-acetyl-3-oxo-2,3-dihydro-1H-benzo[f]indole-2-} carboxylate (3o). ${ }^{1} \mathrm{H} \mathrm{NMR}\left(400 \mathrm{MHz}, \mathrm{CDCl}_{3}\right) \delta 9.10-8.23(\mathrm{~m}, 2 \mathrm{H})$, 8.01-7.80 (m, 2H), 7.69-7.54 (m, 1H), 7.53-7.37 (m, 1H), 5.28-4.98 $(\mathrm{m}, 1 \mathrm{H}), 3.87(\mathrm{~s}, 3 \mathrm{H}), 2.89-2.15(\mathrm{~m}, 3 \mathrm{H})$.

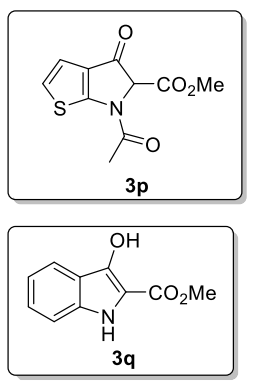

Methyl 6-acetyl-4-oxo-5,6-dihydro-4H-thieno[2,3-b]pyrrole-5carboxylate (3p). ${ }^{1} \mathrm{H}$ NMR $\left(400 \mathrm{MHz}, \mathrm{CDCl}_{3}\right) \delta 7.00(\mathrm{~d}, J=5.6 \mathrm{~Hz}$, $1 \mathrm{H}), 6.94(\mathrm{~d}, J=5.6 \mathrm{~Hz}, 1 \mathrm{H}), 5.25(\mathrm{~s}, 1 \mathrm{H}), 3.90$ (s, 3H), 2.20 (s, 3H). Methyl 3-oxoindoline-2-carboxylate (3q) ${ }^{1} \mathrm{H}$ NMR $(400 \mathrm{MHz}$, $\left.\mathrm{CDCl}_{3}\right) \delta 7.81(\mathrm{~s}, 1 \mathrm{H}), 7.75(\mathrm{~d}, J=8.0 \mathrm{~Hz}, 1 \mathrm{H}), 7.35(\mathrm{t}, J=8.0 \mathrm{~Hz}, 1 \mathrm{H})$, $7.27(\mathrm{~d}, J=7.6 \mathrm{~Hz}, 1 \mathrm{H}), 7.10(\mathrm{t}, J=7.6 \mathrm{~Hz}, 1 \mathrm{H}), 3.97(\mathrm{~s}, 3 \mathrm{H})$.

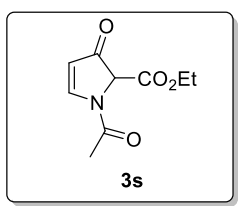

Ethyl 1-acetyl-3-oxo-2,3-dihydro-1H-pyrrole-2-carboxylate (3s). tautomeric mixture of $3 \mathrm{~s}$. the major ${ }^{1} \mathrm{H}$ NMR $\left(400 \mathrm{MHz}, \mathrm{CDCl}_{3}\right) \delta 8.98$ $(\mathrm{d}, J=2.8 \mathrm{~Hz}, 1 \mathrm{H}), 5.70(\mathrm{~d}, J=2.8 \mathrm{~Hz}, 1 \mathrm{H}), 4.93(\mathrm{~s}, 1 \mathrm{H}), 4.13(\mathrm{q}, J=$ $6.0 \mathrm{~Hz}, 2 \mathrm{H}), 2.31(\mathrm{~s}, 3 \mathrm{H}), 1.17(\mathrm{t}, J=6.8 \mathrm{~Hz}, 3 \mathrm{H})$.

2.2 The compounds $\mathbf{4 b - 4 z}$ were synthesized according to the known literature procedures. ${ }^{[2]}$ 

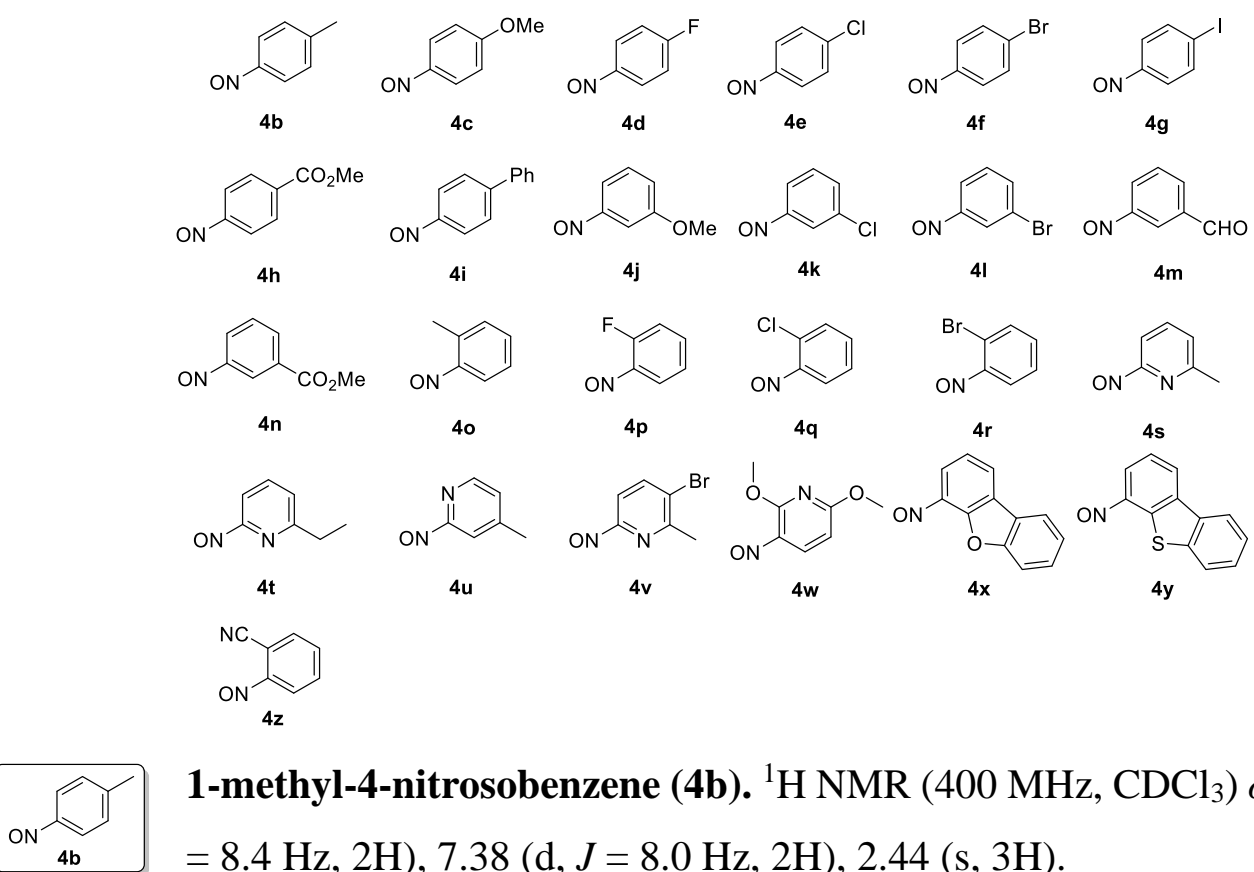

1-methyl-4-nitrosobenzene (4b). ${ }^{1} \mathrm{H}$ NMR $\left(400 \mathrm{MHz}, \mathrm{CDCl}_{3}\right) \delta 7.80(\mathrm{~d}, J$ $=8.4 \mathrm{~Hz}, 2 \mathrm{H}), 7.38(\mathrm{~d}, J=8.0 \mathrm{~Hz}, 2 \mathrm{H}), 2.44(\mathrm{~s}, 3 \mathrm{H})$.

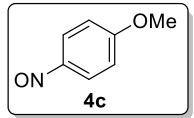

1-methoxy-4-nitrosobenzene (4c). ${ }^{1} \mathrm{H}$ NMR $\left(400 \mathrm{MHz}, \mathrm{CDCl}_{3}\right) \delta 7.90$ $(\mathrm{d}, J=6.4 \mathrm{~Hz}, 2 \mathrm{H}), 7.01(\mathrm{~d}, J=9.2 \mathrm{~Hz}, 2 \mathrm{H}), 3.93$ (s, 3H).

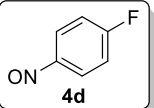

1-fluoro-4-nitrosobenzene (4d). ${ }^{1} \mathrm{H}$ NMR $\left(400 \mathrm{MHz}, \mathrm{CDCl}_{3}\right) \delta 8.01-7.91$ $(\mathrm{m}, 2 \mathrm{H}), 7.31-7.23(\mathrm{~m}, 2 \mathrm{H})$.

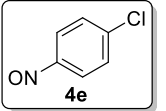

1-chloro-4-nitrosobenzene (4e). ${ }^{1} \mathrm{H}$ NMR $\left(400 \mathrm{MHz}, \mathrm{CDCl}_{3}\right) \delta 7.85(\mathrm{~d}, J$ $=8.4 \mathrm{~Hz}, 2 \mathrm{H}), 7.59(\mathrm{~d}, J=8.4 \mathrm{~Hz}, 2 \mathrm{H})$.

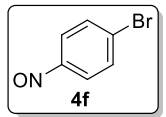

1-bromo-4-nitrosobenzene (4f). ${ }^{1} \mathrm{H} \mathrm{NMR}\left(400 \mathrm{MHz}, \mathrm{CDCl}_{3}\right) \delta 7.77$ (s, $4 \mathrm{H})$.

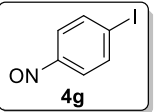

1-iodo-4-nitrosobenzene (4g). ${ }^{1} \mathrm{H}$ NMR $\left(400 \mathrm{MHz}, \mathrm{CDCl}_{3}\right) \delta 8.03(\mathrm{~d}, J=$ $8.4 \mathrm{~Hz}, 2 \mathrm{H}), 7.60(\mathrm{~d}, J=8.8 \mathrm{~Hz}, 2 \mathrm{H})$.

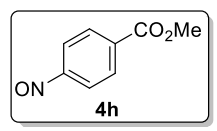

Methyl 4-nitrosobenzoate (4h). ${ }^{1} \mathrm{H}$ NMR $\left(400 \mathrm{MHz}, \mathrm{CDCl}_{3}\right) \delta 8.29(\mathrm{~d}$, $J=8.8 \mathrm{~Hz}, 2 \mathrm{H}), 7.93(\mathrm{~d}, J=8.4 \mathrm{~Hz}, 2 \mathrm{H}), 3.98(\mathrm{~s}, 3 \mathrm{H})$.

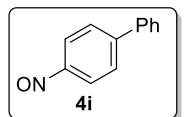

4-nitroso-1,1'-biphenyl (4i). ${ }^{1} \mathrm{H}$ NMR $\left(400 \mathrm{MHz}, \mathrm{CDCl}_{3}\right) \delta 7.98(\mathrm{~d}, J=$ $8.4 \mathrm{~Hz}, 2 \mathrm{H}), 7.83(\mathrm{~d}, J=8.4 \mathrm{~Hz}, 2 \mathrm{H}), 7.67(\mathrm{~d}, J=6.8 \mathrm{~Hz}, 2 \mathrm{H}), 7.55-7.41$ $(\mathrm{m}, 3 \mathrm{H})$.

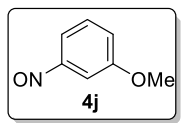

1-methoxy-3-nitrosobenzene (4j). ${ }^{1} \mathrm{H}$ NMR $\left(400 \mathrm{MHz}, \mathrm{CDCl}_{3}\right) \delta 8.02$ (d, $J=7.6 \mathrm{~Hz}, 1 \mathrm{H}), 7.59(\mathrm{t}, J=8.0 \mathrm{~Hz}, 1 \mathrm{H}), 7.30-7.26(\mathrm{~m}, 1 \mathrm{H}), 6.92-6.86(\mathrm{~m}$, $1 \mathrm{H}), 3.85(\mathrm{~s}, 3 \mathrm{H})$.

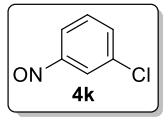

1-chloro-3-nitrosobenzene (4k). ${ }^{1} \mathrm{H}$ NMR $\left(400 \mathrm{MHz}, \mathrm{CDCl}_{3}\right) \delta$ 8.09-8.03 $(\mathrm{m}, 1 \mathrm{H})$, 7.71-7.67 (m, 1H), 7.66-7.60 (m, 2H). 

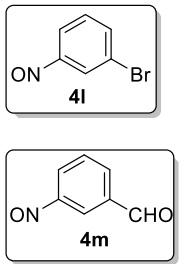

1-bromo-3-nitrosobenzene (4l). ${ }^{1} \mathrm{H}$ NMR (400 MHz, $\left.\mathrm{CDCl}_{3}\right) \delta$ 8.15-8.07 (m, 1H), 7.86-7.81 (m, 1H), 7.79-7.74 (m, 1H), 7.57 (t, $J=7.6 \mathrm{~Hz}, 1 \mathrm{H})$.

3-nitrosobenzaldehyde (4m). ${ }^{1} \mathrm{H}$ NMR (400 MHz, $\left.\mathrm{CDCl}_{3}\right) \delta 10.19(\mathrm{~s}$, $1 \mathrm{H}), 8.37(\mathrm{~s}, 1 \mathrm{H}), 8.26(\mathrm{~d}, J=7.6 \mathrm{~Hz}, 1 \mathrm{H}), 8.14(\mathrm{~d}, J=7.6 \mathrm{~Hz}, 1 \mathrm{H}), 7.83$ (t, $J=7.6 \mathrm{~Hz}, 1 \mathrm{H})$.

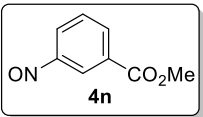

Methyl 3-nitrosobenzoate (4n). ${ }^{1} \mathrm{H}$ NMR $\left(400 \mathrm{MHz}, \mathrm{CDCl}_{3}\right) \delta 8.59$ (s, 1H), 8.44-8.31 (m, 1H), 8.04-7.95 (m, 1H), 7.70 (t, $J=7.6 \mathrm{~Hz}, 1 \mathrm{H}), 3.99$ $(\mathrm{s}, 3 \mathrm{H})$.

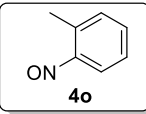

1-methyl-2-nitrosobenzene (4o). ${ }^{1} \mathrm{H} \mathrm{NMR}\left(400 \mathrm{MHz}, \mathrm{CDCl}_{3}\right) \delta 7.59(\mathrm{t}, J$ $=8.4 \mathrm{~Hz}, 1 \mathrm{H}), 3.34(\mathrm{~s}, 3 \mathrm{H})$.

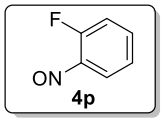

1-fluoro-2-nitrosobenzene (4p). ${ }^{1} \mathrm{H}$ NMR $\left(400 \mathrm{MHz}, \mathrm{CDCl}_{3}\right) \delta 7.77-7.67$ (m, 1H), 7.55-7.46 (m, 1H), $7.14(\mathrm{t}, J=7.6 \mathrm{~Hz}, 1 \mathrm{H}), 6.52-6.45(\mathrm{~m}, 1 \mathrm{H})$.

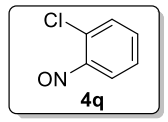

1-chloro-2-nitrosobenzene (4q). ${ }^{1} \mathrm{H}$ NMR $\left(400 \mathrm{MHz}, \mathrm{CDCl}_{3}\right) \delta 7.77(\mathrm{~d}, J$ $=8.0 \mathrm{~Hz}, 1 \mathrm{H}), 7.67-7.57(\mathrm{~m}, 1 \mathrm{H}), 7.26-7.19(\mathrm{~m}, 1 \mathrm{H}), 6.26-6.14(\mathrm{~m}, 1 \mathrm{H})$.

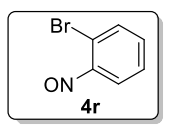

1-bromo-2-nitrosobenzene (4r). ${ }^{1} \mathrm{H}$ NMR $\left(400 \mathrm{MHz}, \mathrm{CDCl}_{3}\right) \delta 7.99(\mathrm{~d}, J$ $=8.0 \mathrm{~Hz}, 1 \mathrm{H}), 7.59-7.48(\mathrm{~m}, 1 \mathrm{H}), 7.32-7.26(\mathrm{~m}, 1 \mathrm{H}), 6.25-6.15(\mathrm{~m}, 1 \mathrm{H})$.

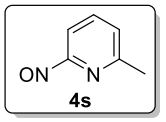

2-methyl-6-nitrosopyridine (4s). ${ }^{1} \mathrm{H}$ NMR (400 MHz, $\left.\mathrm{CDCl}_{3}\right)$ monomer $\delta 7.94(\mathrm{t}, J=7.6 \mathrm{~Hz}, 1 \mathrm{H}), 7.51(\mathrm{~d}, J=7.6 \mathrm{~Hz}, 1 \mathrm{H}), 7.25(\mathrm{~d}, J=7.6 \mathrm{~Hz}, 1 \mathrm{H})$, $2.71(\mathrm{~s}, 3 \mathrm{H})$, dimmer $\delta 7.75(\mathrm{t}, J=8.0 \mathrm{~Hz}, 1 \mathrm{H}), 7.56(\mathrm{~d}, J=8.0 \mathrm{~Hz}, 1 \mathrm{H}), 7.12(\mathrm{~d}, J=$ $7.6 \mathrm{~Hz}, 1 \mathrm{H}), 2.12(\mathrm{~s}, 3 \mathrm{H})$.

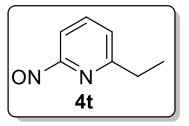

2-ethyl-6-nitrosopyridine (4t). ${ }^{1} \mathrm{H}$ NMR (400 MHz, $\left.\mathrm{CDCl}_{3}\right)$ monomer $\delta$ $7.94(\mathrm{t}, J=7.6 \mathrm{~Hz}, 1 \mathrm{H}), 7.54(\mathrm{~d}, J=7.6 \mathrm{~Hz}, 1 \mathrm{H}), 7.10(\mathrm{~d}, J=7.6 \mathrm{~Hz}, 1 \mathrm{H})$, $3.00(\mathrm{q}, J=7.6 \mathrm{~Hz}, 2 \mathrm{H}), 1.38(\mathrm{t}, J=7.6 \mathrm{~Hz}, 3 \mathrm{H})$. dimmer $\delta 7.77(\mathrm{t}, J=7.6 \mathrm{~Hz}, 1 \mathrm{H})$, $7.62(\mathrm{~d}, J=8.0 \mathrm{~Hz}, 1 \mathrm{H}), 7.10(\mathrm{~d}, J=7.6 \mathrm{~Hz}, 1 \mathrm{H}), 2.40(\mathrm{q}, J=7.6 \mathrm{~Hz}, 2 \mathrm{H}), 0.72(\mathrm{t}, J=$ $7.6 \mathrm{~Hz}, 3 \mathrm{H})$.

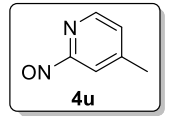

4-methyl-2-nitrosopyridine (4u). ${ }^{1} \mathrm{H} \mathrm{NMR}\left(400 \mathrm{MHz}, \mathrm{CDCl}_{3}\right) \delta 7.81(\mathrm{~d}$, $J=4.8 \mathrm{~Hz}, 1 \mathrm{H}), 7.58(\mathrm{~s}, 1 \mathrm{H}), 7.06(\mathrm{~d}, J=4.8 \mathrm{~Hz}, 1 \mathrm{H}), 2.39(\mathrm{~s}, 3 \mathrm{H})$.

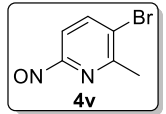

3-bromo-2-methyl-6-nitrosopyridine (4v). ${ }^{1} \mathrm{H} \mathrm{NMR}\left(400 \mathrm{MHz}, \mathrm{CDCl}_{3}\right)$ monomer $\delta 8.16(\mathrm{~d}, J=8.4 \mathrm{~Hz}, 1 \mathrm{H}), 6.97(\mathrm{~d}, J=8.0 \mathrm{~Hz}, 1 \mathrm{H}), 2.86(\mathrm{~s}, 3 \mathrm{H})$, dimmer $\delta 8.02(\mathrm{~d}, J=8.4 \mathrm{~Hz}, 1 \mathrm{H}), 7.54(\mathrm{~d}, J=8.4 \mathrm{~Hz}, 1 \mathrm{H}), 2.27$ (s, 3H). 

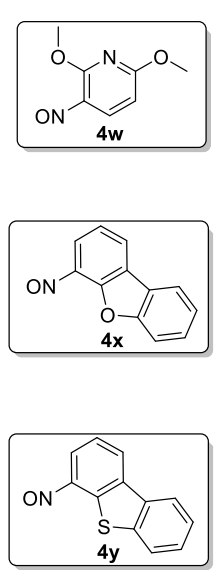
$4 y$

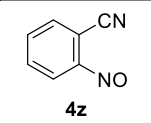

2,6-dimethoxy-3-nitrosopyridine (4w). ${ }^{1} \mathrm{H} \mathrm{NMR}\left(400 \mathrm{MHz}, \mathrm{CDCl}_{3}\right) \delta$ $6.84(\mathrm{~d}, J=8.8 \mathrm{~Hz}, 1 \mathrm{H}), 6.21(\mathrm{~d}, J=8.8 \mathrm{~Hz}, 1 \mathrm{H}), 4.35(\mathrm{~s}, 3 \mathrm{H}), 4.09(\mathrm{~s}$, $3 \mathrm{H})$.

4-nitrosodibenzo[b, $\boldsymbol{d}]$ furan (4x). ${ }^{1} \mathrm{H}$ NMR $\left(400 \mathrm{MHz}, \mathrm{CDCl}_{3}\right) \delta 8.28$ $(\mathrm{d}, J=7.6 \mathrm{~Hz}, 1 \mathrm{H}), 8.02(\mathrm{~d}, J=8.0 \mathrm{~Hz}, 1 \mathrm{H}), 7.79(\mathrm{~d}, J=8.4 \mathrm{~Hz}, 1 \mathrm{H})$, $7.59(\mathrm{t}, J=7.6 \mathrm{~Hz}, 2 \mathrm{H}), 7.52-7.42(\mathrm{~m}, 2 \mathrm{H})$.

4-nitrosodibenzo $[\boldsymbol{b}, \boldsymbol{d}]$ thiophene $(\mathbf{4 y}) .{ }^{1} \mathrm{H} \mathrm{NMR}\left(400 \mathrm{MHz}, \mathrm{CDCl}_{3}\right) \delta$ $9.60(\mathrm{~d}, J=7.6 \mathrm{~Hz}, 1 \mathrm{H}), 8.52(\mathrm{~d}, J=7.6 \mathrm{~Hz}, 1 \mathrm{H}), 8.24-8.17(\mathrm{~m}, 1 \mathrm{H})$, 8.00-7.91 (m, 2H), 7.58-7.49 (m, 2H).

2-nitrosobenzonitrile. ${ }^{1} \mathrm{H} \mathrm{NMR}\left(400 \mathrm{MHz}, \mathrm{CDCl}_{3}\right) \delta 8.06(\mathrm{~d}, J=7.6 \mathrm{~Hz}$, $1 \mathrm{H}), 7.85(\mathrm{t}, J=7.5 \mathrm{~Hz}, 1 \mathrm{H}), 7.76(\mathrm{t}, J=8.0 \mathrm{~Hz}, 1 \mathrm{H}), 7.00(\mathrm{~d}, J=8.0 \mathrm{~Hz}$, $1 \mathrm{H})$.

\section{Preparation of the Compounds $3 \mathbf{c}^{[3]}, \mathbf{3 r} \mathbf{r}^{[4]}$ and $3 \mathbf{t}^{[5]}$}

3.1 General procedures for the synthesis of $\mathbf{3} \mathbf{c}^{[3]}$

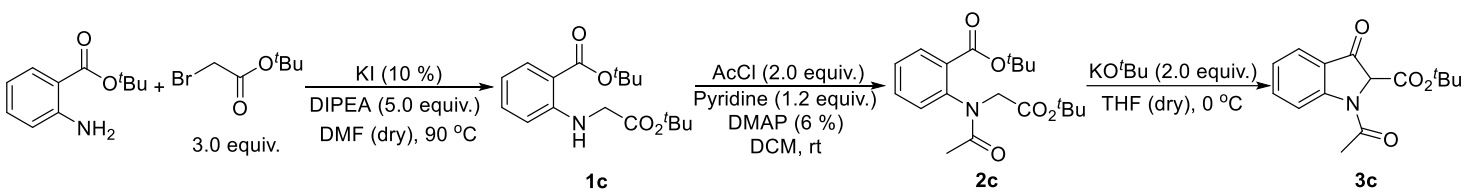

tert-Butyl 2-aminobenzoate (1.00 g, $5.0 \mathrm{mmol})$, tert-butyl 2-bromoacetate (2.90 g, 3.0 equiv.) and dry DMF (20 mL) were added in a dried round flask, then $\mathrm{KI}(0.08 \mathrm{~g}$, 10 mol \%), DIPEA (3.23g, 5.0 equiv.) was added into the flask. The reaction mixture was stirred at $90{ }^{\circ} \mathrm{C}$ (oil bath) until tert-Butyl 2-aminobenzoate disappeared detected by TLC. Then the reaction mixture was cooling to room temperature, and was quenched by addition of saturated ammonium chloride solution. The mixture was extracted with EA $(40 \mathrm{~mL} \times 2)$. The collected organic layers were washed with water and brine, dried over $\mathrm{Na}_{2} \mathrm{SO}_{4}$, filtered and concentrated under reduced pressure. The crude product was purified by column chromatography on silica gel with $\mathrm{V}_{\mathrm{PE}}: \mathrm{V}_{\mathrm{EA}}=30: 1$ as the eluent to afford the product 1c as a colorless liquid in $88 \%$ yield. tert-Butyl 2-((2-(tert-butoxy)2-oxoethyl)amino)benzoate (1c). ${ }^{1} \mathrm{H}$ NMR $\left(400 \mathrm{MHz}, \mathrm{CDCl}_{3}\right) \delta 8.19$ (s, 1H), $7.88(\mathrm{~d}$, $J=7.6 \mathrm{~Hz}, 1 \mathrm{H}), 7.32(\mathrm{t}, J=7.6 \mathrm{~Hz}, 1 \mathrm{H}), 6.61(\mathrm{t}, J=7.6 \mathrm{~Hz}, 1 \mathrm{H}), 6.50(\mathrm{~d}, J=8.4 \mathrm{~Hz}$, $1 \mathrm{H}), 3.88(\mathrm{~d}, J=5.2 \mathrm{~Hz}, 2 \mathrm{H}), 1.59(\mathrm{~s}, 9 \mathrm{H}), 1.50(\mathrm{~s}, 9 \mathrm{H}) .{ }^{13} \mathrm{C} \mathrm{NMR}\left(100 \mathrm{MHz}, \mathrm{CDCl}_{3}\right)$ $\delta 170.0,168.0,150.0,134.1,132.0,115.2,112.7,111.2,82.0,80.8,45.9,28.4,28.2$. HRMS (ESI) m/z: [M + Na] $]^{+}$calcd for $\mathrm{C}_{17} \mathrm{H}_{25} \mathrm{NO}_{4} \mathrm{Na} 330.1676$; found 330.1687. IR 
$(\mathrm{KBr}, v) 3334,2975,2929,1730,1680,1575,1520,1369,1242,1149,1124,1073,849$, $751 \mathrm{~cm}^{-1}$.

The compound $1 \mathrm{c}(1.35 \mathrm{~g}, 4.4 \mathrm{mmol})$ was treated with pyridine $(0.42 \mathrm{~g}, 1.2$ equiv.) and DMAP (0.03 g, $6 \mathrm{~mol} \%)$ followed by addition of dichloromethane as solvent. The reaction mixture was stirred at room temperature, then Acetylchloride ( $0.69 \mathrm{~g}, 2.0$ equiv.) was added dropwise to the stirred mixture. After the starting material completed conversion which detected by TLC, the reaction was quenched by addition of saturated ammonium chloride solution. The mixture was extracted with DCM $(40 \mathrm{~mL} \times 3)$. The collected organic layers were dried over $\mathrm{Na}_{2} \mathrm{SO}_{4}$, filtered and concentrated under reduced pressure. The crude product was purified by column chromatography on silica gel with $\mathrm{V}_{\mathrm{PE}}: \mathrm{V}_{\mathrm{EA}}=7: 1$ as the eluent to afford the product $2 \mathrm{c}$ as a colorless liquid in $82 \%$ yield. tert-butyl 2-( $N$-(2-(tert-butoxy)-2-oxoethyl)acetamido)benzoate $(2 \mathrm{c}) .{ }^{1} \mathrm{H}$ NMR (400 MHz, $\left.\mathrm{CDCl}_{3}\right) \delta 7.81(\mathrm{~d}, J=8.0 \mathrm{~Hz}, 1 \mathrm{H}), 7.57(\mathrm{~d}, J=8.0 \mathrm{~Hz}, 1 \mathrm{H}), 7.48(\mathrm{~d}$, $J=7.6 \mathrm{~Hz}, 1 \mathrm{H}), 7.37(\mathrm{~d}, J=7.6 \mathrm{~Hz}, 1 \mathrm{H}), 4.84(\mathrm{~d}, J=17.2 \mathrm{~Hz}, 1 \mathrm{H}), 3.40(\mathrm{~d}, J=16.8$ $\mathrm{Hz}, 1 \mathrm{H}), 1.75$ (s, 3H), 1.49 (s, 9H), 1.39 (s, 9H). $\left.{ }^{13} \mathrm{C} \mathrm{NMR} \mathrm{(100} \mathrm{MHz,} \mathrm{CDCl}_{3}\right) \delta 170.2$, 168.4, 165.3, 142.0, 132.7, 131.3, 131.2, 130.7, 128.6, 82.5, 81.6, 51.8, 28.1, 22.0. HRMS (ESI) m/z: $[\mathrm{M}+\mathrm{Na}]^{+}$calcd for $\mathrm{C}_{19} \mathrm{H}_{27} \mathrm{NO}_{5} \mathrm{Na} 372.1781$; found 372.1795. IR $(\mathrm{KBr}, v)$ 3474, 2980, 2935, 1745, 1717, 1674, 1370, 1302, 1159, 1125, $847 \mathrm{~cm}^{-1}$.

To a solution of $\mathrm{KO}^{t} \mathrm{Bu}(0.81 \mathrm{~g}, 2.0$ equiv. $)$ in dry $\mathrm{THF}(20 \mathrm{~mL})$ at $0{ }^{\circ} \mathrm{C}$ was added $2 \mathrm{c}(1.26 \mathrm{~g}, 3.6 \mathrm{mmol})$ in THF $(5 \mathrm{~mL})$ dropwise. After $10 \mathrm{~min}$, the starting material $2 \mathrm{c}$ completed conversion. The reaction was quenched by addition of saturated ammonium chloride solution, and the THF was removed by reduced pressure and the resulting mixture was extracted with EA $(30 \mathrm{~mL} \times 3)$. The collected organic layers were dried over $\mathrm{Na}_{2} \mathrm{SO}_{4}$, filtered and concentrated under reduced pressure. The crude product was purified by column chromatography on silica gel with $\mathrm{V}_{\mathrm{PE}}: \mathrm{V}_{\mathrm{EA}}=10: 1$ as the eluent to afford the product $3 \mathbf{c}$ as a white solide in $82 \%$ yield. tert-Butyl 1-acetyl-3-oxoindoline2-carboxylate (3c). m.p. $121-122^{\circ} \mathrm{C} .{ }^{1} \mathrm{H}$ NMR $\left(400 \mathrm{MHz}, \mathrm{CDCl}_{3}\right) \delta 8.51(\mathrm{~d}, J=8.0 \mathrm{~Hz}$, 1H), 7.80-7.30 (m, 2H), 7.15 (t, $J=7.6 \mathrm{~Hz}, 1 \mathrm{H}), 4.97-4.70(\mathrm{~m}, 1 \mathrm{H}), 2.73-2.07$ (m, 3H), $1.44(\mathrm{~s}, 9 \mathrm{H}) .{ }^{13} \mathrm{C} \mathrm{NMR}\left(100 \mathrm{MHz}, \mathrm{CDCl}_{3}\right) \delta 189.5,168.3,163.3,153.9,137.8,124.5$, 124.4, 123.0, 118.5, 84.5, 69.3, 27.8, 23.7. HRMS (ESI) m/z: $[\mathrm{M}+\mathrm{Na}]^{+}$calcd for $\mathrm{C}_{15} \mathrm{H}_{17} \mathrm{NO}_{4} \mathrm{Na}$ 298.1050; found 298.1054. IR (KBr, v) 3454, 3010, 2976, 2931, 1720 , $1688,1465,1390,1349,1305,1252,1151,1003,773 \mathrm{~cm}^{-1}$.

3.2 General procedures for the synthesis of $3 r^{[4]}$ 


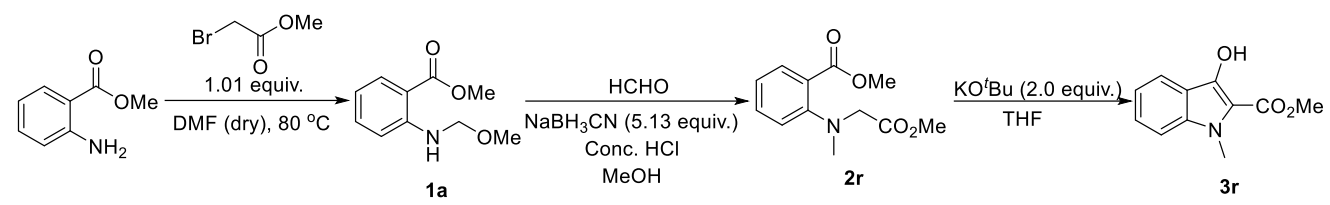

Methyl 2-((methoxymethyl)amino)benzoate $\mathbf{1 a}^{[1]}(0.39 \mathrm{~g}, 2.0 \mathrm{mmol})$ and sodium cyanoborohydride ( $0.64 \mathrm{~g}, 5.13$ equiv.) were added in a round flask. The flask was degassed via vacuum and backfilled with argon gas. The purge and backfilled process was repeated three times. Then $\mathrm{MeOH}(15.0 \mathrm{~mL}), \mathrm{HCHO}$ aqueous solution $(4.0 \mathrm{~mL})$ and conc. $\mathrm{HCl}(1.0 \mathrm{ml})$ were added to the flask, and the reaction mixture was stirred at room temperature until the starting material completed conversion. Then the reaction was quenched by addition of saturated $\mathrm{NaHCO}_{3}$ solution, the solvent $\mathrm{MeOH}$ was removed by reduced pressure and the resulting mixture was extracted with EA $(20 \mathrm{~mL}$ $\times 3$ ). The collected organic layers were washed with saturated $\mathrm{NaHCO}_{3}$ solution and brine, dried over $\mathrm{Na}_{2} \mathrm{SO}_{4}$, filtered and concentrated under reduced pressure. The crude product was purified by column chromatography on silica gel with $\mathrm{V}_{\mathrm{PE}}$ : $\mathrm{V}_{\mathrm{EA}}(4: 1)$ as the eluent to afford the product $\mathbf{2} \mathbf{r}$ as a colorless liquid in $98 \%$ yield. Methyl 2-((2methoxy-2-oxoethyl)(methyl)amino)benzoate (2r). ${ }^{1} \mathrm{H}$ NMR $\left(400 \mathrm{MHz}, \mathrm{CDCl}_{3}\right) \delta$ $7.71(\mathrm{~d}, J=6.8 \mathrm{~Hz}, 1 \mathrm{H}), 7.37(\mathrm{t}, J=7.2 \mathrm{~Hz}, 1 \mathrm{H}), 7.02(\mathrm{~d}, J=8.4 \mathrm{~Hz}, 1 \mathrm{H}), 6.91(\mathrm{t}, J=$ $7.2 \mathrm{~Hz}, 1 \mathrm{H}), 3.89$ (s, 2H), 3.86 (s, 3H), 3.74 (s, 3H), 3.00 (s, 3H). ${ }^{13} \mathrm{C}$ NMR (100 MHz, $\left.\mathrm{CDCl}_{3}\right) \delta 171.4,168.5,151.3,132.6,131.9,121.6,120.3,118.8,57.9,52.2,51.8,41.1$. HRMS (ESI) m/z: $[\mathrm{M}+\mathrm{Na}]^{+}$calcd for $\mathrm{C}_{12} \mathrm{H}_{15} \mathrm{NO}_{4} \mathrm{Na} 260.0893$; found 260.0891. IR $(\mathrm{KBr}, v)$ 3455, 2954, 2922, 2852, 1748, 1719, 1492, 1434, 1261, 1201, 1122, 1078, 757 $\mathrm{cm}^{-1}$.

To a solution of $\mathrm{KO}^{t} \mathrm{Bu}(0.43 \mathrm{~g}, 2.0$ equiv. $)$ in dry $\mathrm{THF}(20 \mathrm{~mL})$ at $0{ }^{\circ} \mathrm{C}$ was added $2 \mathbf{r}(0.45 \mathrm{~g}, 1.9 \mathrm{mmol})$ in THF $(5 \mathrm{~mL})$ dropwise. After $10 \mathrm{~min}$, the starting material $\mathbf{2 r}$ completed conversion. The reaction was quenched by addition of saturated ammonium chloride solution, and the THF was removed by reduced pressure and the resulting mixture was extracted with EA $(30 \mathrm{~mL} \times 3)$. The collected organic layers were dried over $\mathrm{Na}_{2} \mathrm{SO}_{4}$, filtered and concentrated under reduced pressure. The crude product was purified by column chromatography on silica gel with $\mathrm{V}_{\mathrm{PE}}: \mathrm{V}_{\mathrm{EA}}=10: 1$ as the eluent to afford the product 3r as a white solid in 77\% yield. Methyl 3-hydroxy-1-methyl-1Hindole-2-carboxylate (3r). m.p. $146-147{ }^{\circ} \mathrm{C} .{ }^{1} \mathrm{H}$ NMR $\left(400 \mathrm{MHz}, \mathrm{CDCl}_{3}\right) \delta 8.59$ (s, $1 \mathrm{H}), 7.75(\mathrm{~d}, J=8.4 \mathrm{~Hz}, 1 \mathrm{H}), 7.38(\mathrm{t}, J=7.6 \mathrm{~Hz}, 1 \mathrm{H}), 7.25(\mathrm{t}, J=9.2 \mathrm{~Hz}, 1 \mathrm{H}), 7.07(\mathrm{t}$, $J=7.6 \mathrm{~Hz}, 1 \mathrm{H}), 3.99$ (s, 3H), 3.86 (s, 3H). ${ }^{13} \mathrm{C} \mathrm{NMR}\left(100 \mathrm{MHz}, \mathrm{CDCl}_{3}\right) \delta 164.8,148.8$, 
137.9, 127.4, 120.4, 119.2, 116.6, 109.9, 109.5, 51.6, 31.8. HRMS (ESI) m/z: [M - H] $]^{-}$ calcd for $\mathrm{C}_{11} \mathrm{H}_{10} \mathrm{NO}_{3}$ 204.0655; found 204.0669. IR (KBr, v) 3305, 3059, 2953, 1655, $1545,1462,1373,1304,1237,1168,1123,983,738 \mathrm{~cm}^{-1}$.

3.3 General procedures for the synthesis of $3 \mathbf{t}^{[5]}$

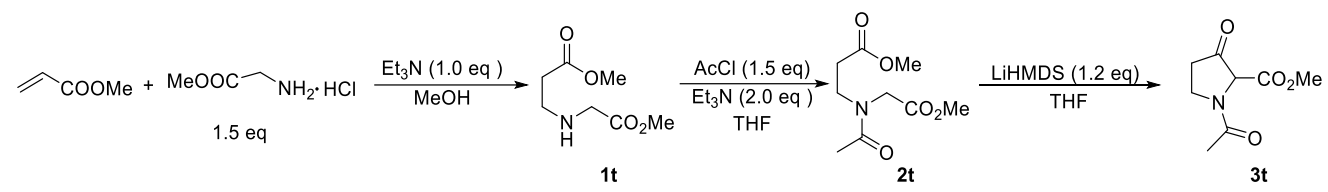

methyl acrylate $(1.72 \mathrm{~g}, 20 \mathrm{mmol})$ and $\mathrm{Et}_{3} \mathrm{~N}(2.02 \mathrm{~g}, 20 \mathrm{mmol})$ were added to a stirred solution of glycine methyl ester hydrochloride (3.75 g, $30 \mathrm{mmol})$ in $\mathrm{MeOH}$ (30 $\mathrm{mL})$. The resulting solution was stirred at room temperature for two days and then the solvent $\mathrm{MeOH}$ was removed under reduced pressure. EA $(30 \mathrm{~mL})$ and water $(30 \mathrm{~mL})$ were added to the resulting residue, and the aqueous layer was extracted with EtOAc $(30 \mathrm{~mL} \times 2)$. The combined organic layers were dried over $\mathrm{Na}_{2} \mathrm{SO}_{4}$, filtered and concentrated under reduced pressure. The crude product was purified by column chromatography on silica gel with $\mathrm{V}_{\mathrm{PE}}: \mathrm{V}_{\mathrm{EA}}(1: 2)$ as the eluent to afford the product $1 \mathrm{t}$ as a colorless liquid in 69\% yield. Methyl 3-((2-methoxy-2oxoethyl)amino)propanoate (1t). ${ }^{1} \mathrm{H}$ NMR $\left(400 \mathrm{MHz}, \mathrm{CDCl}_{3}\right) \delta 3.57$ (s, 3H), 3.53 (s, $3 \mathrm{H}), 3.27(\mathrm{~s}, 2 \mathrm{H}), 2.74(\mathrm{t}, J=6.4 \mathrm{~Hz}, 2 \mathrm{H}), 2.36(\mathrm{t}, J=6.4 \mathrm{~Hz}, 2 \mathrm{H}) .{ }^{13} \mathrm{C}$ NMR (100 MHz, $\left.\mathrm{CDCl}_{3}\right) \delta 172.6,172.5,51.5,51.4,50.4,44.5,34.4$. HRMS (ESI) m/z: $[\mathrm{M}+\mathrm{H}]^{+}$calcd for $\mathrm{C}_{7} \mathrm{H}_{14} \mathrm{NO}_{4}$ 176.0917; found 176.0904. IR ( $\left.\mathrm{KBr}, v\right)$ 3452, 2956, 1740, 1438, 1208 , $1177,1149 \mathrm{~cm}^{-1}$.

To a solution of $1 \mathrm{t}(2.45 \mathrm{~g}, 14 \mathrm{mmol})$ and triethylamine ( $2.83 \mathrm{~g}, 2.0$ equiv.) in anhydrous THF $(60 \mathrm{~mL})$ under $\mathrm{N}_{2}$ atmosphere at $0{ }^{\circ} \mathrm{C}$ was added acetyl chloride $(1.65$ $\mathrm{g}, 1.5$ equiv.) dropwise. After stirring at room temperature for $1 \mathrm{~h}$, water was added to the reaction mixture at $0{ }^{\circ} \mathrm{C}$. The THF was removed under reduced pressure and the crude residue was extracted with ethyl acetate $(20 \mathrm{~mL} \times 3)$. The combined organic layers were washed with saturated aqueous $\mathrm{Na}_{2} \mathrm{CO}_{3}$, brine, dried over anhydrous $\mathrm{Na}_{2} \mathrm{SO}_{4}$, filtered and concentrated under reduced pressure. The crude product was purified by column chromatography on silica gel with $\mathrm{V}_{\mathrm{PE}}: \mathrm{V}_{\mathrm{EA}}(1: 2)$ as the eluent to afford the product $\mathbf{2 t}$ as a colorless liquid in 76\% yield. Methyl 3-(N-(2-methoxy-2oxoethyl)acetamido)propanoate (2t). ${ }^{1} \mathrm{H} \mathrm{NMR}\left(400 \mathrm{MHz}, \mathrm{CDCl}_{3}\right) \delta$ 4.12-3.84 (m, $2 \mathrm{H}), 3.63-3.46(\mathrm{~m}, 8 \mathrm{H}), 2.52(\mathrm{t}, J=6.4 \mathrm{~Hz}, 2 \mathrm{H}), 2.11-1.83(\mathrm{~m}, 3 \mathrm{H}) .{ }^{13} \mathrm{C}$ NMR $(100$ 
$\left.\mathrm{MHz}, \mathrm{CDCl}_{3}\right) \delta 171.4,170.9,169.8,51.9,51.8,47.7,45.3,33.4,20.8$. HRMS (ESI) $\mathrm{m} / \mathrm{z}:[\mathrm{M}+\mathrm{Na}]^{+}$calcd for $\mathrm{C}_{9} \mathrm{H}_{15} \mathrm{NO}_{5} \mathrm{Na} 240.0842$; found 240.0853. IR ( $\left.\mathrm{KBr}, v\right) 3455$, $2956,1740,1655,1438,1370,1211,1178,1001 \mathrm{~cm}^{-1}$.

A solution of $2 \mathbf{t}(2.38 \mathrm{~g}, 11 \mathrm{mmol})$ in THF $(12 \mathrm{~mL})$ was added dropwise to a stirred and cooled $\left(-78{ }^{\circ} \mathrm{C}\right)$ solution of $\left(\mathrm{Me}_{3} \mathrm{Si}\right)_{2} \mathrm{NLi}(13.2 \mathrm{~mL}, 1.0 \mathrm{M}$ in THF) in dry THF (36 $\mathrm{mL}$ ) under $\mathrm{N}_{2}$ atmosphere. The cold bath was left in place, but not recharged, and stirring was continued for $2.5 \mathrm{~h}$, by which time the temperature had risen to $-30{ }^{\circ} \mathrm{C}$. the reaction mixture stirred at $-30{ }^{\circ} \mathrm{C}$ until the starting material completed conversion. Then hydrochloric acid $(1 \mathrm{~N}, 20 \mathrm{~mL})$ was poured into the reaction mixture to quench the reaction. The THF was removed under reduced pressure and the crude residue was extracted with ethyl acetate $(30 \mathrm{~mL} \times 3)$. The combined organic extracts were dried $\left(\mathrm{Na}_{2} \mathrm{SO}_{4}\right)$ filtered and concentrated under reduced pressure. The crude product was purified by column chromatography on silica gel with $\mathrm{V}_{\mathrm{PE}}$ : $\mathrm{V}_{\mathrm{EA}}(1: 2)$ as the eluent to afford the product 3t as a light yellow liquid in 18\% yield. Methyl 1-acetyl-3oxopyrrolidine-2-carboxylate (3t). ${ }^{1} \mathrm{H}$ NMR (400 MHz, $\left.\mathrm{CDCl}_{3}\right) \delta 4.69-4.57(\mathrm{~m}, 1 \mathrm{H})$, 4.21-3.86 (m, 2H), 3.82-3.71 (m, 3H), 2.85-2.60 (m, 2H), 2.15-1.91 (m, 3H). ${ }^{13} \mathrm{C}$ NMR $\left(100 \mathrm{MHz} \mathrm{CDCl}_{3}\right) \delta 203.4,170.3,166.0,65.0,53.2,43.4,37.3,21.0$. HRMS (ESI) $\mathrm{m} / \mathrm{z}:[\mathrm{M}+\mathrm{Na}]^{+}$calcd for $\mathrm{C}_{8} \mathrm{H}_{11} \mathrm{NO}_{4} \mathrm{Na} 208.0580$; found 208.0587. IR $(\mathrm{KBr}, v) 3463$, 2958, 2924, 1774, 1743, 1656, 1438, 1419, 1208, 1178, $1140 \mathrm{~cm}^{-1}$.

\section{General Procedure for the Synthesis of 5aa-5sa, 5ab-5az and 6ta.}

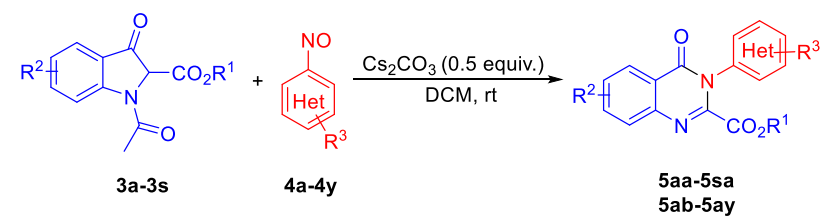

A solution of $3 \mathbf{a}(0.4 \mathrm{mmol})$, nitrobenzene (1.2 equiv.) and $\mathrm{Cs}_{2} \mathrm{CO}_{3}(0.065 \mathrm{~g}, 0.5$ equiv.) in DCM (2 mL) were stirred at room temperature until the starting material completed conversion. The reaction mixture concentrated under reduced pressure. The crude product was purified by column chromatography on silica gel with $\mathrm{V}_{\mathrm{PE}}: \mathrm{V}_{\mathrm{EA}}$ $(8: 1)$ as the eluent to afford the product 5aa, 5ba-5ya, 5ab-5az, 5sa and 6ta was synthesized with the similar experimental procedure. 
Methyl 4-oxo-3-phenyl-3,4-dihydroquinazoline-2-carboxylate (5aa).

Following the general procedure to synthesis the title compound. The pure production 5aa was isolated by flash chromatography column with $\mathrm{V}_{\mathrm{PE}}$ : $\mathrm{V}_{\mathrm{EA}}(8: 1)$ as the eluent. White solid, $108 \mathrm{mg}, 96 \%$ yield; m.p. $148-149{ }^{\circ} \mathrm{C} .{ }^{1} \mathrm{H}$ NMR (400 MHz, DMSO-d6) $\delta 8.21(\mathrm{~d}, J=8.0 \mathrm{~Hz}, 1 \mathrm{H}), 7.98-7.91(\mathrm{~m}, 1 \mathrm{H}), 7.82(\mathrm{~d}, J$ $=8.0 \mathrm{~Hz}, 1 \mathrm{H}), 7.69(\mathrm{t}, J=7.6 \mathrm{~Hz}, 1 \mathrm{H}), 7.57-7.50(\mathrm{~m}, 3 \mathrm{H}), 7.49-7.42(\mathrm{~m}, 2 \mathrm{H}), 3.55(\mathrm{~s}$, $3 \mathrm{H}) .{ }^{13} \mathrm{C}$ NMR (100 MHz, DMSO-d6) $\delta$ 160.9, 160.1, 146.8, 146.1, 135.9, 135.2, 129.4, 129.1, 128.7, 128.4, 127.7, 126.7, 122.0, 53.0. HRMS (ESI) m/z: $[\mathrm{M}+\mathrm{H}]^{+}$calcd for $\mathrm{C}_{16} \mathrm{H}_{13} \mathrm{~N}_{2} \mathrm{O}_{3}$ 281.0921; found 281.0938. IR (KBr, v) 3052, 2950, 2845, 1754, 1679, $1607,1590,1461,1364,1247,1205,783,762,700 \mathrm{~cm}^{-1}$.

Ethyl 4-oxo-3-phenyl-3,4-dihydroquinazoline-2-carboxylate (5ba). Following the general procedure to synthesis the title compound. The pure production $5 \mathbf{b a}$ was isolated by flash chromatography column with $\mathrm{V}_{\mathrm{PE}}$ : $\mathrm{V}_{\mathrm{EA}}(8: 1)$ as the eluent. Pale yellow solid, $108 \mathrm{mg}$, 92\% yield; m.p. 107-110 ${ }^{\circ} \mathrm{C} .{ }^{1} \mathrm{H}$ NMR (400 MHz, $\left.\mathrm{CDCl}_{3}\right) \delta 8.33(\mathrm{~d}, J=8.0 \mathrm{~Hz}, 1 \mathrm{H}), 7.86-7.77(\mathrm{~m}, 2 \mathrm{H}), 7.61-7.53(\mathrm{~m}$, 1H), 7.53-7.45 (m, 3H), $7.36(\mathrm{~d}, J=7.2 \mathrm{~Hz}, 2 \mathrm{H}), 4.07(\mathrm{q}, J=7.2 \mathrm{~Hz}, 2 \mathrm{H}), 0.98(\mathrm{t}, J=$ $7.2 \mathrm{~Hz}, 3 \mathrm{H}) .{ }^{13} \mathrm{C} \mathrm{NMR}\left(100 \mathrm{MHz}, \mathrm{CDCl}_{3}\right) \delta 160.9,160.9,147.3,146.6,136.1,135.0$, 129.6, 129.4, 128.6, 128.2, 127.3, 122.2, 62.8, 13.5. HRMS (ESI) m/z: $[\mathrm{M}+\mathrm{H}]^{+}$calcd for $\mathrm{C}_{17} \mathrm{H}_{15} \mathrm{~N}_{2} \mathrm{O}_{3}$ 295.1077; found 295.1088. IR (KBr, v) 3066, 2972, 1745, 1702, 1688, $1608,1590,1464,1376,1246,1191,1016,770,694 \mathrm{~cm}^{-1}$.

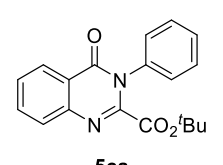

tert-Butyl 4-oxo-3-phenyl-3,4-dihydroquinazoline-2-carboxylate (5ca). Following the general procedure to synthesis the title compound. The pure production $\mathbf{5 c a}$ was isolated by flash chromatography column with $\mathrm{V}_{\mathrm{PE}}: \mathrm{V}_{\mathrm{EA}}$ (8:1) as the eluent. Pale yellow solid, $120 \mathrm{mg}$, 93\% yield; m.p. 127-129 ${ }^{\circ} \mathrm{C} .{ }^{1} \mathrm{H}$ NMR $\left(400 \mathrm{MHz}, \mathrm{CDCl}_{3}\right) \delta 8.30(\mathrm{~d}, J=8.0 \mathrm{~Hz}, 1 \mathrm{H}), 7.84-7.75(\mathrm{~m}, 2 \mathrm{H}), 7.56-$ $7.47(\mathrm{~m}, 4 \mathrm{H}), 7.36(\mathrm{~d}, J=7.6 \mathrm{~Hz}, 2 \mathrm{H}), 1.17(\mathrm{~s}, 9 \mathrm{H}) .{ }^{13} \mathrm{C} \mathrm{NMR}\left(100 \mathrm{MHz}, \mathrm{CDCl}_{3}\right) \delta$ 161.0, 159.9, 147.9, 146.8, 136.2, 134.9, 129.6, 129.4, 128.7, 128.2, 128.2, 127.2, 122.1, 85.0, 27.3. HRMS (ESI) m/z: $[\mathrm{M}+\mathrm{Na}]^{+}$calcd for $\mathrm{C}_{19} \mathrm{H}_{18} \mathrm{~N}_{2} \mathrm{O}_{3} \mathrm{Na}$ 345.1210; found 345.1213. IR (KBr, v) 3062, 2983, 2935, 1734, 1687, 1607, 1589, 1462, 1370, 1253 , $1164,773,757,693 \mathrm{~cm}^{-1}$. 
Methyl 6-methyl-4-oxo-3-phenyl-3,4-dihydroquinazoline-2carboxylate (5da). Following the general procedure to synthesis the

title compound. The pure production 5da was isolated by flash 5da chromatography column with $\mathrm{V}_{\mathrm{PE}}: \mathrm{V}_{\mathrm{EA}}(7: 1)$ as the eluent. Pale yellow solid, $114 \mathrm{mg}$, 97\% yield; m.p. $136-138{ }^{\circ} \mathrm{C} .{ }^{1} \mathrm{H}$ NMR $\left(400 \mathrm{MHz}, \mathrm{CDCl}_{3}\right) \delta 8.11(\mathrm{~s}, 1 \mathrm{H}), 7.72$ (d, $J=$ $8.0 \mathrm{~Hz}, 1 \mathrm{H}), 7.62(\mathrm{~d}, J=8.4 \mathrm{~Hz}, 1 \mathrm{H}), 7.53-7.43(\mathrm{~m}, 3 \mathrm{H}), 7.33(\mathrm{~d}, J=7.2 \mathrm{~Hz}, 2 \mathrm{H}), 3.62$ $(\mathrm{s}, 3 \mathrm{H}), 2.50(\mathrm{~s}, 3 \mathrm{H}) .{ }^{13} \mathrm{C} \mathrm{NMR}\left(100 \mathrm{MHz}, \mathrm{CDCl}_{3}\right) \delta 161.4,160.9,146.1,144.4,139.3$, 136.4, 136.3, 129.5, 129.4, 128.1, 128.0, 126.8, 122.0, 53.2, 21.5. HRMS (ESI) m/z: $[\mathrm{M}+\mathrm{H}]^{+}$calcd for $\mathrm{C}_{17} \mathrm{H}_{15} \mathrm{~N}_{2} \mathrm{O}_{3}$ 295.1077; found 295.1088. IR (KBr, v) 3056, 2951, 2924, 2847, 1741, 1694, 1586, 1487, 1358, 1250, 1209, 839, 795, 745, $694 \mathrm{~cm}^{-1}$.

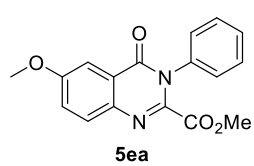

Methyl 6-methoxy-4-oxo-3-phenyl-3,4-dihydroquinazoline-2carboxylate (5ea). Following the general procedure to synthesis the title compound. The pure production 5ea was isolated by flash chromatography column with $\mathrm{V}_{\mathrm{PE}}: \mathrm{V}_{\mathrm{EA}}(7: 1)$ as the eluent. Pale yellow solid, $115 \mathrm{mg}$, 93\% yield; m.p. $157-158{ }^{\circ} \mathrm{C} .{ }^{1} \mathrm{H}$ NMR $\left(400 \mathrm{MHz}, \mathrm{CDCl}_{3}\right) \delta 7.74(\mathrm{~d}, J=8.8 \mathrm{~Hz}, 1 \mathrm{H})$, 7.66 (s, 1H), 7.54-7.43 (m, 3H), 7.38 (d, $J=8.8 \mathrm{~Hz}, 1 \mathrm{H}), 7.33$ (d, $J=7.6 \mathrm{~Hz}, 2 \mathrm{H}), 3.90$ (s, 3H), $3.62(\mathrm{~s}, 3 \mathrm{H}) .{ }^{13} \mathrm{C}$ NMR $\left(100 \mathrm{MHz}, \mathrm{CDCl}_{3}\right) \delta 161.4,160.8,159.9,144.6,140.7$, 136.4, 129.9, 129.5, 129.4, 128.0, 125.0, 123.3, 106.9, 56.0, 53.2. HRMS (ESI) m/z: $[\mathrm{M}+\mathrm{H}]^{+}$calcd for $\mathrm{C}_{17} \mathrm{H}_{15} \mathrm{~N}_{2} \mathrm{O}_{4}$ 311.1026; found 311.1037. IR (KBr, v) 3069, 3028, 2955, 2835, 1741, 1683, 1615, 1486, 1363, 1225, 1027, 845, 748, $695 \mathrm{~cm}^{-1}$.

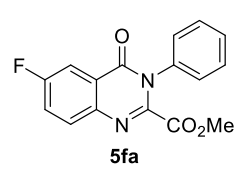

Methyl 6-fluoro-4-oxo-3-phenyl-3,4-dihydroquinazoline-2carboxylate (5fa). Following the general procedure to synthesis the title compound. The pure production $\mathbf{5 f a}$ was isolated by flash chromatography column with $\mathrm{V}_{\mathrm{PE}}: \mathrm{V}_{\mathrm{EA}}(7: 1)$ as the eluent. Pale yellow solid, $111 \mathrm{mg}$, 93\% yield; m.p. $109-111{ }^{\circ} \mathrm{C} .{ }^{1} \mathrm{H}$ NMR $\left(400 \mathrm{MHz}, \mathrm{CDCl}_{3}\right) \delta 8.01-7.91(\mathrm{~m}, 1 \mathrm{H}), 7.84$ $(\mathrm{dd}, J=8.8,4.8 \mathrm{~Hz}, 1 \mathrm{H}), 7.57-7.45(\mathrm{~m}, 4 \mathrm{H}), 7.33(\mathrm{~d}, J=7.2 \mathrm{~Hz}, 2 \mathrm{H}), 3.63(\mathrm{~s}, 3 \mathrm{H}) .{ }^{13} \mathrm{C}$ NMR $\left(100 \mathrm{MHz}, \mathrm{CDCl}_{3}\right) \delta 163.3,162.3\left(J_{C-F}=213 \mathrm{~Hz}\right), 160.8,160.2,146.3,143.1$, $135.9,130.8\left(J_{C-F}=8 \mathrm{~Hz}\right), 129.8,129.6,128.0,123.6\left(J_{C-F}=24 \mathrm{~Hz}\right), 112.5\left(J_{C-F}=24\right.$ $\mathrm{Hz}$ ), 53.3. ${ }^{19} \mathrm{~F}$ NMR $\left(376 \mathrm{MHz}, \mathrm{CDCl}_{3}\right)$-109.5. HRMS (ESI) $\mathrm{m} / \mathrm{z}:[\mathrm{M}+\mathrm{H}]^{+}$calcd for $\mathrm{C}_{16} \mathrm{H}_{12} \mathrm{~N}_{2} \mathrm{O}_{3} \mathrm{~F}$ 299.0826; found 299.0833. IR (KBr, v) 3062, 3039, 2953, 1749, 1691, $1605,1590,1485,1366,1222,1008,856,752,696 \mathrm{~cm}^{-1}$. 


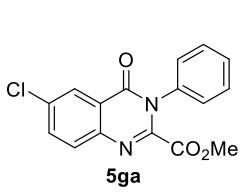

Methyl 6-chloro-4-oxo-3-phenyl-3,4-dihydroquinazoline-2carboxylate (5ga). Following the general procedure to synthesis the title compound. The pure production 5ga was isolated by flash chromatography column with $\mathrm{V}_{\mathrm{PE}}: \mathrm{V}_{\mathrm{EA}}(7: 1)$ as the eluent. Pale yellow solid, $123 \mathrm{mg}$, 98\% yield; m.p. $166-168{ }^{\circ} \mathrm{C} .{ }^{1} \mathrm{H}$ NMR (400 MHz, $\left.\mathrm{CDCl}_{3}\right) \delta 8.28(\mathrm{~s}, 1 \mathrm{H}), 7.81-7.70(\mathrm{~m}$, 2H), 7.54-7.45 (m, 3H), $7.33(\mathrm{~d}, J=6.8 \mathrm{~Hz}, 2 \mathrm{H}), 3.62(\mathrm{~s}, 3 \mathrm{H}) .{ }^{13} \mathrm{C}$ NMR $(100 \mathrm{MHz}$, $\left.\mathrm{CDCl}_{3}\right) \delta 161.1,159.9,147.1,145.0,135.8,135.5,134.7,129.9,129.8,129.6,128.0$, 126.8, 123.4, 53.4. HRMS (ESI) m/z: $[\mathrm{M}+\mathrm{H}]^{+}$calcd for $\mathrm{C}_{16} \mathrm{H}_{12} \mathrm{~N}_{2} \mathrm{O}_{3} \mathrm{Cl}$ 315.0531; found 315.0546. IR (KBr, v) 3067, 3040, 2953, 1747, 1690, 1588, 1469, 1359, 1233, 1206, 1007, 859, 790, 757, $701 \mathrm{~cm}^{-1}$.

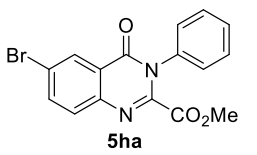

Methyl 6-bromo-4-oxo-3-phenyl-3,4-dihydroquinazoline-2carboxylate (5ha). Following the general procedure to synthesis the title compound. The pure production 5ha was isolated by flash chromatography column with $\mathrm{V}_{\mathrm{PE}}: \mathrm{V}_{\mathrm{EA}}(7: 1)$ as the eluent. Pale yellow solid, $142 \mathrm{mg}$, 98\% yield; m.p. $186-187{ }^{\circ} \mathrm{C} .{ }^{1} \mathrm{H}$ NMR $\left(400 \mathrm{MHz}, \mathrm{CDCl}_{3}\right) \delta 8.43$ (s, 1H), 7.89 (d, $J=$ $8.8 \mathrm{~Hz}, 1 \mathrm{H}), 7.68(\mathrm{~d}, J=8.8 \mathrm{~Hz}, 1 \mathrm{H}), 7.54-7.46(\mathrm{~m}, 3 \mathrm{H}), 7.32(\mathrm{~d}, J=7.6 \mathrm{~Hz}, 2 \mathrm{H}), 3.62$ (s, 3H). ${ }^{13} \mathrm{C}$ NMR $\left(100 \mathrm{MHz}, \mathrm{CDCl}_{3}\right) \delta 161.1,159.7,147.2,145.3,138.3,135.8,130.0$, 129.9, 129.8, 129.6, 127.9, 123.6, 122.5, 53.3. HRMS (ESI) m/z: $[\mathrm{M}+\mathrm{H}]^{+}$calcd for $\mathrm{C}_{16} \mathrm{H}_{12} \mathrm{~N}_{2} \mathrm{O}_{3} \mathrm{Br}$ 359.0026; found 359.0033. IR (KBr, v) 3084, 3066, 2950, 1743, 1699, $1584,1443,1359,1236,1207,837,755,697 \mathrm{~cm}^{-1}$.

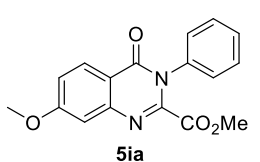

Methyl 7-methoxy-4-oxo-3-phenyl-3,4-dihydroquinazoline-2carboxylate (5ia). Following the general procedure to synthesis the title compound. The pure production 5ia was isolated by flash chromatography column with $\mathrm{V}_{\mathrm{PE}}: \mathrm{V}_{\mathrm{EA}}(7: 1)$ as the eluent. Pale yellow solid, $118 \mathrm{mg}$, 95\% yield; m.p. $130-133{ }^{\circ} \mathrm{C} .{ }^{1} \mathrm{H}$ NMR $\left(400 \mathrm{MHz}, \mathrm{CDCl}_{3}\right) \delta 8.19(\mathrm{~d}, J=8.8 \mathrm{~Hz}, 1 \mathrm{H})$, 7.51-7.41 (m, 3H), $7.32(\mathrm{~d}, J=7.6 \mathrm{~Hz}, 2 \mathrm{H}), 7.18(\mathrm{~s}, 1 \mathrm{H}), 7.11(\mathrm{~d}, J=8.8 \mathrm{~Hz}, 1 \mathrm{H}), 3.89$ (s, 3H), 3.61 (s, 3H). $\left.{ }^{13} \mathrm{C} \mathrm{NMR} \mathrm{(100} \mathrm{MHz,} \mathrm{CDCl}_{3}\right) \delta 165.0,161.3,160.4,148.7,147.5$, 136.1, 129.5, 129.3, 128.8, 128.0, 118.5, 115.5, 109.0, 55.8, 53.2. HRMS (ESI) m/z: $[\mathrm{M}+\mathrm{H}]^{+}$calcd for $\mathrm{C}_{17} \mathrm{H}_{15} \mathrm{~N}_{2} \mathrm{O}_{4}$ 311.1026; found 311.1039. IR ( $\left.\mathrm{KBr}, v\right)$ 3082, 3059, 3035, 2981, 2956, 2845, 1754, 1690, 1608, 1591, 1490, 1438, 1374, 1230, 1017, 859, $782,695 \mathrm{~cm}^{-1}$. 


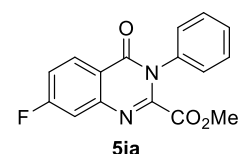

$5 \mathrm{ja}$

carboxylate (5ja). Following the general procedure to synthesis the title compound. The pure production $\mathbf{5 j a}$ was isolated by flash chromatography column with $\mathrm{V}_{\mathrm{PE}}: \mathrm{V}_{\mathrm{EA}}(7: 1)$ as the eluent. White solid, $114 \mathrm{mg}, 96 \%$ yield; m.p. $144-147{ }^{\circ} \mathrm{C} .{ }^{1} \mathrm{H}$ NMR (400 MHz, $\left.\mathrm{CDCl}_{3}\right) \delta 8.37-8.29$ (m, 1H), 7.54-7.42 $(\mathrm{m}, 4 \mathrm{H}), 7.37-7.26(\mathrm{~m}, 3 \mathrm{H}), 3.62(\mathrm{~s}, 3 \mathrm{H}) .{ }^{13} \mathrm{C} \mathrm{NMR}\left(100 \mathrm{MHz}, \mathrm{CDCl}_{3}\right) \delta 166.8\left(J_{C-F}=\right.$ $254 \mathrm{~Hz}), 161.1,160.2,148.7\left(J_{C-F}=13 \mathrm{~Hz}\right), 148.1,135.8,130.2\left(J_{C-F}=11 \mathrm{~Hz}\right), 129.8$, 129.5, 128.0, $119.0\left(J_{C-F}=2 \mathrm{~Hz}\right), 117.4\left(J_{C-F}=23 \mathrm{~Hz}\right), 113.7\left(J_{C-F}=22 \mathrm{~Hz}\right), 53.3 .{ }^{19} \mathrm{~F}$ NMR (376 MHz, $\mathrm{CDCl}_{3}$ ) -109.5. HRMS (ESI) m/z: $[\mathrm{M}+\mathrm{H}]^{+}$calcd for $\mathrm{C}_{16} \mathrm{H}_{12} \mathrm{~N}_{2} \mathrm{O}_{3} \mathrm{~F}$ 299.0826; found 299.0827. IR ( $\mathrm{KBr}, v)$ 3058, 3044, 2953, 2927, 1748, 1688, 1590, $1485,1359,1216,1008,857,809,750,697 \mathrm{~cm}^{-1}$.

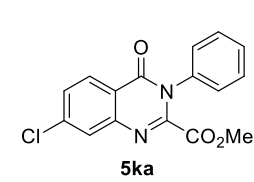

Methyl 7-chloro-4-oxo-3-phenyl-3,4-dihydroquinazoline-2carboxylate (5ka). Following the general procedure to synthesis the title compound. The pure production 5ka was isolated by flash chromatography column with $\mathrm{V}_{\mathrm{PE}}: \mathrm{V}_{\mathrm{EA}}(7: 1)$ as the eluent. White solid, $121 \mathrm{mg}, 96 \%$ yield; m.p. 86-87 ${ }^{\circ} \mathrm{C} .{ }^{1} \mathrm{H}$ NMR (400 MHz, $\left.\mathrm{CDCl}_{3}\right) \delta 8.28(\mathrm{~d}, J=8.8 \mathrm{~Hz}, 1 \mathrm{H}), 7.82(\mathrm{~d}$, $J=1.6 \mathrm{~Hz}, 1 \mathrm{H}), 7.57-7.47(\mathrm{~m}, 4 \mathrm{H}), 7.37-7.30(\mathrm{~m}, 2 \mathrm{H}), 3.64(\mathrm{~s}, 3 \mathrm{H}) .{ }^{13} \mathrm{C}$ NMR $(100$ $\left.\mathrm{MHz}, \mathrm{CDCl}_{3}\right) \delta 161.0,160.3,148.1,147.5,141.4,135.8,129.8,129.6,129.3,128.8$, 128.0, 127.8, 120.7, 53.3. HRMS (ESI) m/z: $[\mathrm{M}+\mathrm{H}]^{+}$calcd for $\mathrm{C}_{16} \mathrm{H}_{12} \mathrm{~N}_{2} \mathrm{O}_{3} \mathrm{Cl}$ 315.0531; found 315.0547. IR (KBr, v) 3058, 3003, 2952, 1748, 1686, 1602, 1587, $1438,1361,1242,920,781,761,701,690 \mathrm{~cm}^{-1}$.

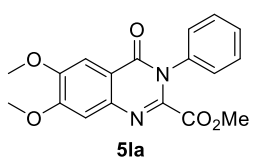

Methyl 6,7-dimethoxy-4-oxo-3-phenyl-3,4-dihydroquinazoline-2carboxylate (5la). Following the general procedure to synthesis the title compound. The pure production 5la was isolated by flash chromatography column with $\mathrm{V}_{\mathrm{PE}}: \mathrm{V}_{\mathrm{EA}}(7: 1)$ as the eluent. White solid, $123 \mathrm{mg}, 90 \%$ yield; m.p. 197-199 ${ }^{\circ} \mathrm{C} .{ }^{1} \mathrm{H} \mathrm{NMR}\left(400 \mathrm{MHz}, \mathrm{CDCl}_{3}\right) \delta 7.61$ (s, 1H), 7.52-7.44 (m, 3H), $7.32(\mathrm{~d}, J=7.6 \mathrm{~Hz}, 2 \mathrm{H}), 7.21(\mathrm{~s}, 1 \mathrm{H}), 3.99(\mathrm{~s}, 3 \mathrm{H}), 3.98(\mathrm{~s}, 3 \mathrm{H}), 3.62(\mathrm{~s}, 3 \mathrm{H}) .{ }^{13} \mathrm{C} \mathrm{NMR}$ $\left(100 \mathrm{MHz}, \mathrm{CDCl}_{3}\right) \delta 161.4,160.3,155.4,150.5,145.5,142.5,136.5,129.5,129.4$, 128.0, 115.8, 108.7, 106.2, 56.5, 53.2. HRMS (ESI) m/z: $[\mathrm{M}+\mathrm{H}]^{+}$calcd for $\mathrm{C}_{18} \mathrm{H}_{17} \mathrm{~N}_{2} \mathrm{O}_{5}$ 341.1132; found 341.1137. IR (KBr, v) 3067, 3003, 2950, 2838, 1742, $1677,1611,1502,1391,1236,1210,1149,997,883,843,752,695 \mathrm{~cm}^{-1}$. 


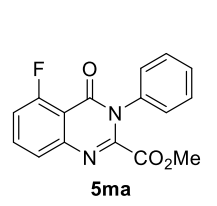

carboxylate (5ma). Following the general procedure to synthesis the

title compound. The pure production $\mathbf{5 m a}$ was isolated by flash chromatography column with $\mathrm{V}_{\mathrm{PE}}: \mathrm{V}_{\mathrm{EA}}(7: 1)$ as the eluent. Pale yellow solid, $84 \mathrm{mg}$, 69\% yield; m.p. 196-198 ${ }^{\circ} \mathrm{C} .{ }^{1} \mathrm{H}$ NMR $\left(400 \mathrm{MHz}, \mathrm{CDCl}_{3}\right) \delta$ 7.80-7.71 (m, 1H), $7.62(\mathrm{~d}$, $J=8.4 \mathrm{~Hz}, 1 \mathrm{H}), 7.55-7.45(\mathrm{~m}, 3 \mathrm{H}), 7.33(\mathrm{~d}, J=7.6 \mathrm{~Hz}, 2 \mathrm{H}), 7.23(\mathrm{t}, J=9.2 \mathrm{~Hz}, 1 \mathrm{H})$, $3.62(\mathrm{~s}, 3 \mathrm{H}) .{ }^{13} \mathrm{C} \mathrm{NMR}\left(100 \mathrm{MHz}, \mathrm{CDCl}_{3}\right) \delta 161.6\left(J_{C-F}=266 \mathrm{~Hz}\right), 161.1,157.9\left(J_{C-F}\right.$ $=4 \mathrm{~Hz}), 148.5,147.8,135.6\left(J_{C-F}=10 \mathrm{~Hz}\right), 135.6,129.8,129.5,128.2,124.2\left(J_{C-F}=4\right.$ $\mathrm{Hz}), 115.5\left(J_{C-F}=21 \mathrm{~Hz}\right), 53.4 .{ }^{19} \mathrm{~F}$ NMR $\left(376 \mathrm{MHz}, \mathrm{CDCl}_{3}\right)-108.5$. HRMS (ESI) m/z: $[\mathrm{M}+\mathrm{H}]^{+}$calcd for $\mathrm{C}_{16} \mathrm{H}_{12} \mathrm{~N}_{2} \mathrm{O}_{3} \mathrm{~F}$ 299.0826; found 299.0832. IR ( $\left.\mathrm{KBr}, v\right)$ 3067, 2949, 2841, 1752, 1690, 1612, 1470, 1363, 1219, 1023, 826, 739, $702 \mathrm{~cm}^{-1}$.

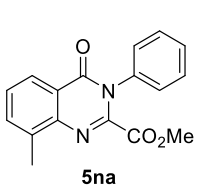

\section{Methyl}

8-methyl-4-oxo-3-phenyl-3,4-dihydroquinazoline-2-

carboxylate (5na). Following the general procedure to synthesis the title compound. The pure production 5na was isolated by flash chromatography column with $\mathrm{V}_{\mathrm{PE}}: \mathrm{V}_{\mathrm{EA}}(7: 1)$ as the eluent. Pale yellow solid, $100 \mathrm{mg}$, $85 \%$ yield; m.p. $136-138{ }^{\circ} \mathrm{C} .{ }^{1} \mathrm{H}$ NMR $\left(400 \mathrm{MHz}, \mathrm{CDCl}_{3}\right) \delta 8.18(\mathrm{~d}, J=8.0 \mathrm{~Hz}, 1 \mathrm{H})$, $7.66(\mathrm{~d}, J=7.2 \mathrm{~Hz}, 1 \mathrm{H}), 7.54-7.43(\mathrm{~m}, 4 \mathrm{H}), 7.35(\mathrm{~d}, J=7.6 \mathrm{~Hz}, 2 \mathrm{H}), 3.60(\mathrm{~s}, 3 \mathrm{H}), 2.66$ (s, 3H). ${ }^{13} \mathrm{C}$ NMR $\left(100 \mathrm{MHz}, \mathrm{CDCl}_{3}\right) \delta 161.7,161.3,145.7,145.2,137.1,136.2,135.7$, 129.6, 129.5, 128.2, 128.1, 125.0, 122.3, 53.0, 17.4. HRMS (ESI) m/z: calcd for $[\mathrm{M}+$ $\mathrm{H}]^{+} \mathrm{C}_{17} \mathrm{H}_{15} \mathrm{~N}_{2} \mathrm{O}_{3}$ 295.1077; found 295.1078. IR (KBr, v) 2953, 2923, 2850, 1748, 1688, 1610, 1590, 1434, 1367, 1241, 1070, 867, 780, 739, $696 \mathrm{~cm}^{-1}$.

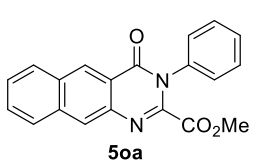

Methyl

4-oxo-3-phenyl-3,4-dihydrobenzo[g]quinazoline-2-

carboxylate (50a). Following the general procedure to synthesis the title compound. The pure production 5oa was isolated by flash chromatography column with $\mathrm{V}_{\mathrm{PE}}: \mathrm{V}_{\mathrm{EA}}(7: 1)$ as the eluent. Pale yellow solid, $128 \mathrm{mg}$, 97\% yield; m.p. $183-185{ }^{\circ} \mathrm{C} .{ }^{1} \mathrm{H}$ NMR (400 MHz, $\left.\mathrm{CDCl}_{3}\right) \delta 8.92(\mathrm{~s}, 1 \mathrm{H}), 8.31(\mathrm{~s}, 1 \mathrm{H})$, $8.06(\mathrm{~d}, J=8.4 \mathrm{~Hz}, 1 \mathrm{H}), 8.01(\mathrm{~d}, J=8.4 \mathrm{~Hz}, 1 \mathrm{H}), 7.65(\mathrm{t}, J=7.2 \mathrm{~Hz}, 1 \mathrm{H}), 7.58(\mathrm{t}, J=$ 8.0 Hz, 1H), 7.55-7.46 (m, 3H), $7.39(\mathrm{~d}, J=7.6 \mathrm{~Hz}, 2 \mathrm{H}), 3.66(\mathrm{~s}, 3 \mathrm{H}) .{ }^{13} \mathrm{C}$ NMR $(100$ $\left.\mathrm{MHz}, \mathrm{CDCl}_{3}\right) \delta 161.6,161.5,146.1,141.6,136.8,136.4,132.5,129.6,129.5,129.1$, 129.0, 128.4, 128.3, 127.3, 126.8, 120.4, 53.3. HRMS (ESI) m/z: $[\mathrm{M}+\mathrm{H}]^{+}$calcd for $\mathrm{C}_{20} \mathrm{H}_{15} \mathrm{~N}_{2} \mathrm{O}_{3}$ 331.1077; found 331.1088. IR ( $\left.\mathrm{KBr}, v\right)$ 3057, 2952, 2851, 1739, 1698, $1587,1452,1355,1215,788,751,694 . \mathrm{cm}^{-1}$. 


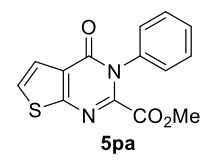

Methyl 4-oxo-3-phenyl-3,4-dihydrothieno[2,3-d] pyrimidine-2carboxylate (5pa). Following the general procedure to synthesis the title compound. The pure production 5pa was isolated by flash chromatography column with $\mathrm{V}_{\mathrm{PE}}: \mathrm{V}_{\mathrm{EA}}(7: 1)$ as the eluent. White solid, $108 \mathrm{mg}, 94 \%$ yield; m.p. 161-163 ${ }^{\circ} \mathrm{C} .{ }^{1} \mathrm{H}$ NMR $\left(400 \mathrm{MHz}, \mathrm{CDCl}_{3}\right) \delta 7.55$ (d, $\left.J=6.0 \mathrm{~Hz}, 1 \mathrm{H}\right), 7.53-$ $7.45(\mathrm{~m}, 3 \mathrm{H}), 7.41(\mathrm{~d}, J=5.6 \mathrm{~Hz}, 1 \mathrm{H}), 7.32(\mathrm{~d}, J=7.6 \mathrm{~Hz}, 2 \mathrm{H}), 3.63(\mathrm{~s}, 3 \mathrm{H}) .{ }^{13} \mathrm{C} \mathrm{NMR}$ $\left(100 \mathrm{MHz}, \mathrm{CDCl}_{3}\right) \delta 161.8,161.0,157.2,146.6,135.8,129.8,129.5,128.0,126.1$, 125.2, 122.9, 53.4. HRMS (ESI) $\mathrm{m} / \mathrm{z}$ : $[\mathrm{M}+\mathrm{H}]^{+}$calcd for $\mathrm{C}_{14} \mathrm{H}_{11} \mathrm{~N}_{2} \mathrm{O}_{3} \mathrm{~S} 287.0485$; found 287.0499. IR (KBr, v) 3118, 3013, 2952, 2924, 2853, 1737, 1697, 1549, 1433, 1355, $1294,1210,954,730,701 \mathrm{~cm}^{-1}$.

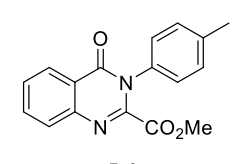

Methyl 4-oxo-3-(p-tolyl)-3,4-dihydroquinazoline-2-carboxylate (5ab). Following the general procedure to synthesis the title compound. $5 a b$

The pure production 5ab was isolated by flash chromatography column with $\mathrm{V}_{\mathrm{PE}}$ : $\mathrm{V}_{\mathrm{EA}}(8: 1)$ as the eluent. White solid, $113 \mathrm{mg}, 96 \%$ yield; m.p. 155-156 ${ }^{\circ} \mathrm{C}$. ${ }^{1} \mathrm{H}$ NMR (400 MHz, $\left.\mathrm{CDCl}_{3}\right) \delta 8.32(\mathrm{~d}, J=7.6 \mathrm{~Hz}, 1 \mathrm{H}), 7.84-7.77(\mathrm{~m}, 2 \mathrm{H}), 7.60-7.52$ (m, 1H), 7.29 (d, $J=7.6 \mathrm{~Hz}, 2 \mathrm{H}), 7.21(\mathrm{~d}, J=7.6 \mathrm{~Hz}, 2 \mathrm{H}), 3.65(\mathrm{~s}, 3 \mathrm{H}), 2.40(\mathrm{~s}, 3 \mathrm{H})$. ${ }^{13} \mathrm{C}$ NMR $\left(100 \mathrm{MHz}, \mathrm{CDCl}_{3}\right) \delta 161.4,161.0,147.1,146.5,139.7,135.0,133.3,130.1$, 128.6, 128.2, 127.7, 127.3, 122.3, 53.3, 21.3. HRMS (ESI) m/z: $[\mathrm{M}+\mathrm{H}]^{+}$calcd for $\mathrm{C}_{17} \mathrm{H}_{15} \mathrm{~N}_{2} \mathrm{O}_{3}$ 295.1077; found 295.1074. IR (KBr, v) 3073, 3042, 3000, 2950, 2923, $1740,1686,1596,1513,1463,1359,1248,1206,1003,856,771,760,696 \mathrm{~cm}^{-1}$.

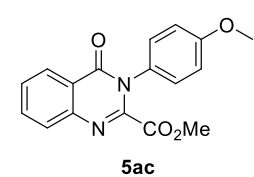

Methyl 3-(4-methoxyphenyl)-4-oxo-3,4-dihydroquinazoline-2carboxylate (5ac). Following the general procedure to synthesis the title compound. The pure production 5ac was isolated by flash chromatography column with $\mathrm{V}_{\mathrm{PE}}: \mathrm{V}_{\mathrm{EA}}(7: 1)$ as the eluent. White solid, $118 \mathrm{mg}, 95 \%$ yield; m.p. $171-172{ }^{\circ} \mathrm{C} .{ }^{1} \mathrm{H}$ NMR $(400 \mathrm{MHz}, \mathrm{CDCl} 3) \delta 8.31$ (d, $\left.J=8.0 \mathrm{~Hz}, 1 \mathrm{H}\right), 7.84-$ $7.75(\mathrm{~m}, 2 \mathrm{H}), 7.60-7.51(\mathrm{~m}, 1 \mathrm{H}), 7.25(\mathrm{~d}, J=8.4 \mathrm{~Hz}, 2 \mathrm{H}), 6.99(\mathrm{~d}, J=8.4 \mathrm{~Hz}, 2 \mathrm{H}), 3.83$ (s, 3H), 3.66 (s, 3H). $\left.{ }^{13} \mathrm{C} \mathrm{NMR} \mathrm{(100} \mathrm{MHz,} \mathrm{CDCl}_{3}\right) \delta 161.4,161.1,160.2,147.3,146.5$, 134.9, 129.2, 128.6, 128.4, 128.1, 127.3, 122.2, 114.6, 55.5, 53.3. HRMS (ESI) m/z: $[\mathrm{M}+\mathrm{H}]^{+}$calcd for $\mathrm{C}_{17} \mathrm{H}_{15} \mathrm{~N}_{2} \mathrm{O}_{4}$ 311.1026; found 311.1014. IR (KBr, v) 3069, 3036, 2960, 2938, 2844, 1747, 1695, 1610, 1510, 1464, 1368, 1257, 1205, 1002, 829, 779, $757,700 \mathrm{~cm}^{-1}$. 


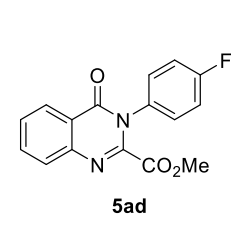

Methyl 3-(4-fluorophenyl)-4-oxo-3,4-dihydroquinazoline-2carboxylate (5ad). Following the general procedure to synthesis the title compound. The pure production 5ad was isolated by flash chromatography column with $\mathrm{V}_{\mathrm{PE}}: \mathrm{V}_{\mathrm{EA}}(8: 1)$ as the eluent. White solid, 115 mg, 97\% yield; m.p. 165-167 ${ }^{\circ} \mathrm{C} .{ }^{1} \mathrm{H}$ NMR (400 MHz, $\left.\mathrm{CDCl}_{3}\right) \delta 8.33$ (d, $J=7.6$ $\mathrm{Hz}, 1 \mathrm{H}), 7.87-7.80(\mathrm{~m}, 2 \mathrm{H})$, 7.64-7.55 (m, 1H), 7.37-7.29 (m, 2H), 7.23-7.16 (m, 2H), $3.69(\mathrm{~s}, 3 \mathrm{H}) .{ }^{13} \mathrm{C} \mathrm{NMR}\left(100 \mathrm{MHz}, \mathrm{CDCl}_{3}\right) \delta 162.6\left(J_{\mathrm{C}-\mathrm{F}}=323 \mathrm{~Hz}\right), 161.5\left(J_{\mathrm{C}-\mathrm{F}}=48 \mathrm{~Hz}\right)$, 146.6, 146.4, 135.2, $131.9\left(J_{\mathrm{C}-\mathrm{F}}=3.2 \mathrm{~Hz}\right), 130.1,130.0,128.9,128.3,127.4,122.1$, $116.6\left(J_{\mathrm{C}-\mathrm{F}}=23 \mathrm{~Hz}\right)$, 53.4. ${ }^{19} \mathrm{~F}$ NMR $\left(376 \mathrm{MHz}, \mathrm{CDCl}_{3}\right)-110.8$. HRMS (ESI) m/z: [M $+\mathrm{H}]^{+}$calcd for $\mathrm{C}_{16} \mathrm{H}_{12} \mathrm{~N}_{2} \mathrm{O}_{3} \mathrm{~F} 299.0826$; found 299.0836. IR (KBr, v) 3122, 3090, 3057, 2953, 1744, 1688, 1609, 1509, 1463, 1365, 1251, 1208, 1006, 837, 782, 760, $698 \mathrm{~cm}^{-1}$.

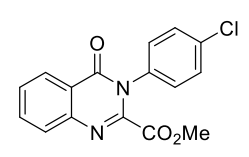

5 ae

\section{Methyl}

carboxylate (5ae). Following the general procedure to synthesis the title compound. The pure production 5ae was isolated by flash chromatography column with $\mathrm{V}_{\mathrm{PE}}: \mathrm{V}_{\mathrm{EA}}(8: 1)$ as the eluent. Light yellow solid, $122 \mathrm{mg}$, 97\% yield; m.p. $176-178{ }^{\circ} \mathrm{C} .{ }^{1} \mathrm{H}$ NMR $\left(400 \mathrm{MHz}, \mathrm{CDCl}_{3}\right) \delta 8.30(\mathrm{~d}, J=8.0 \mathrm{~Hz}, 1 \mathrm{H})$, 8.40-7.77 (m, 2H), 7.61-7.54 (m, 1H), 7.46 (d, $J=8.0 \mathrm{~Hz}, 2 \mathrm{H}), 7.27(\mathrm{~d}, J=8.0 \mathrm{~Hz}, 2 \mathrm{H})$, $3.68(\mathrm{~s}, 3 \mathrm{H}) .{ }^{13} \mathrm{C} \mathrm{NMR}\left(100 \mathrm{MHz}, \mathrm{CDCl}_{3}\right) \delta 161.2,160.8,146.3,135.7,135.2,134.6$, 129.8, 129.4, 128.9, 128.3, 127.4, 122.1, 53.5. HRMS (ESI) m/z: calcd for $[\mathrm{M}+\mathrm{H}]^{+}$ $\mathrm{C}_{16} \mathrm{H}_{12} \mathrm{~N}_{2} \mathrm{O}_{3} \mathrm{Cl}$ 315.0531; found 315.0533. IR (KBr, v) 3094, 3070, 3005, 2953, 1737, 1690, 1604, 1490, 1463, 1360, 1250, 1216, 1092, 932, 826, 774, $695 \mathrm{~cm}^{-1}$.

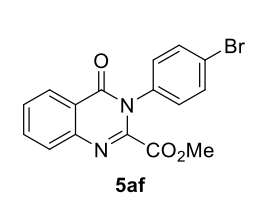

Methyl

3-(4-bromophenyl)-4-oxo-3,4-dihydroquinazoline-2carboxylate (5af). Following the general procedure to synthesis the title compound. The pure production 5af was isolated by flash chromatography column with $\mathrm{V}_{\mathrm{PE}}: \mathrm{V}_{\mathrm{EA}}(7: 1)$ as the eluent. Pale yellow solid, $135 \mathrm{mg}$, 94\% yield; m.p. $175-177{ }^{\circ} \mathrm{C} .{ }^{1} \mathrm{H}$ NMR $\left(400 \mathrm{MHz}, \mathrm{CDCl}_{3}\right) \delta 8.30(\mathrm{~d}, J=8.0 \mathrm{~Hz}, 1 \mathrm{H})$, 7.85-7.77 (m, 2H), $7.62(\mathrm{~d}, J=7.6 \mathrm{~Hz}, 2 \mathrm{H}), 7.60-7.53(\mathrm{~m}, 1 \mathrm{H}), 7.21(\mathrm{~d}, J=7.6 \mathrm{~Hz}, 2 \mathrm{H})$, 3.69 (s, 3H). ${ }^{13} \mathrm{C} \mathrm{NMR}\left(100 \mathrm{MHz}, \mathrm{CDCl}_{3}\right) \delta 161.1,160.7,146.3,146.2,135.2,135.2$, 132.7, 129.7, 128.9, 128.3, 127.4, 123.8, 122.1, 53.5. HRMS (ESI) m/z: $[\mathrm{M}+\mathrm{H}]^{+}$calcd for $\mathrm{C}_{16} \mathrm{H}_{12} \mathrm{~N}_{2} \mathrm{O}_{3} \mathrm{Br}$ 359.0026; found 359.0030. IR (KBr, v) 3091, 3070, 3003, 2952 , 1739, 1690, 1603, 1488, 1360, 1248, 1216, 1017, 1008, 932, 823, 774, $694 \mathrm{~cm}^{-1}$. 


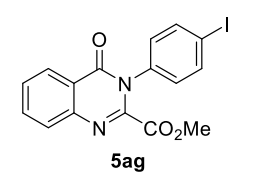

carboxylate (5ag). Following the general procedure to synthesis the title compound. The pure production 5ag was isolated by flash chromatography column with $\mathrm{V}_{\mathrm{PE}}: \mathrm{V}_{\mathrm{EA}}(7: 1)$ as the eluent. White solid, $151 \mathrm{mg}, 93 \%$ yield; m.p. $170-171^{\circ} \mathrm{C} .{ }^{1} \mathrm{H}$ NMR $\left(400 \mathrm{MHz}, \mathrm{CDCl}_{3}\right) \delta 8.30$ (d, $\left.J=8.0 \mathrm{~Hz}, 1 \mathrm{H}\right), 7.89$ $7.75(\mathrm{~m}, 4 \mathrm{H}), 7.63-7.53(\mathrm{~m}, 1 \mathrm{H}), 7.08(\mathrm{~d}, J=8.0 \mathrm{~Hz}, 2 \mathrm{H}), 3.70(\mathrm{~s}, 3 \mathrm{H}) .{ }^{13} \mathrm{C}$ NMR $(100$ $\left.\mathrm{MHz}, \mathrm{CDCl}_{3}\right) \delta 161.1,160.7,146.3,146.2,138.7,135.9,135.2,129.8,129.0,128.3$, 127.4, 122.1, 95.6, 53.5. HRMS (ESI) $\mathrm{m} / \mathrm{z}:[\mathrm{M}+\mathrm{H}]^{+}$calcd for $\mathrm{C}_{16} \mathrm{H}_{12} \mathrm{~N}_{2} \mathrm{O}_{3} \mathrm{I}$ 406.9887; found 406.9899. IR (KBr, v) 3087, 2951, 2851, 1739, 1730, 1694, 1595, 1485, 1463 , $1359,1247,1216,1014,1005,929,820,778,692 \mathrm{~cm}^{-1}$.

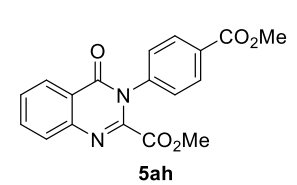

\section{Methyl}

3-(4-(methoxycarbonyl)phenyl)-4-oxo-3,4dihydroquinazoline-2-carboxylate (5ah). Following the general procedure to synthesis the title compound. The pure production 5ah was isolated by flash chromatography column with $\mathrm{V}_{\mathrm{PE}}: \mathrm{V}_{\mathrm{EA}}(5: 1)$ as the eluent. Yellow solid, $129 \mathrm{mg}$, 95\% yield; m.p. 222-224 ${ }^{\circ} \mathrm{C} .{ }^{1} \mathrm{H}$ NMR (400 MHz, $\left.\mathrm{CDCl}_{3}\right) \delta 8.31$ (d, $J=$ $8.0 \mathrm{~Hz}, 1 \mathrm{H}), 8.17(\mathrm{~d}, J=7.6 \mathrm{~Hz}, 2 \mathrm{H}), 7.88-7.78(\mathrm{~m}, 2 \mathrm{H}), 7.60-7.53(\mathrm{~m}, 1 \mathrm{H}), 7.42(\mathrm{~d}, J$ $=7.6 \mathrm{~Hz}, 2 \mathrm{H}), 3.94(\mathrm{~s}, 3 \mathrm{H}), 3.66(\mathrm{~s}, 3 \mathrm{H}) .{ }^{13} \mathrm{C} \mathrm{NMR}\left(100 \mathrm{MHz}, \mathrm{CDCl}_{3}\right) \delta 166.0,161.1$, $160.7,146.3,146.0,140.3,135.3,131.2,130.8,129.0,128.4,128.1,127.4,122.1,53.5$, 52.5. HRMS (ESI) m/z: $[\mathrm{M}+\mathrm{H}]^{+}$calcd for $\mathrm{C}_{18} \mathrm{H}_{15} \mathrm{~N}_{2} \mathrm{O}_{5}$ 339.0975; found 339.0979. IR $(\mathrm{KBr}, v)$ 3041, 3002, 2952, 2849, 1750, 1720, 1686, 1590, 1435, 1366, 1284, 1246, $1113,1007,932,868,776,764,703,696 \mathrm{~cm}^{-1}$.

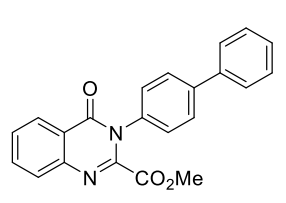

5 ai

Methyl 3-([1,1'-biphenyl]-4-yl)-4-oxo-3,4-dihydroquinazoline-2carboxylate (5ai). Following the general procedure to synthesis the title compound. The pure production 5ai was isolated by flash chromatography column with $\mathrm{V}_{\mathrm{PE}}: \mathrm{V}_{\mathrm{EA}}(8: 1)$ as the eluent. White solid, $138 \mathrm{mg}$, 97\% yield; m.p. 187-188 ${ }^{\circ} \mathrm{C} .{ }^{1} \mathrm{H}$ NMR (400 MHz, $\left.\mathrm{CDCl}_{3}\right) \delta 8.37$ (d, $J=$ 8.0 Hz, 1H), 7.89-7.81 (m, 2H), 7.73 (d, $J=7.6 \mathrm{~Hz}, 2 \mathrm{H}), 7.66-7.57(\mathrm{~m}, 3 \mathrm{H}), 7.51-7.36$ (m, 5H), 3.68 (s, 3H). ${ }^{13} \mathrm{C} \mathrm{NMR}\left(100 \mathrm{MHz}, \mathrm{CDCl}_{3}\right) \delta 161.4,161.0,146.9,146.5,142.6$, 139.8, 135.2, 135.1, 129.0, 128.8, 128.4, 128.3, 128.1, 128.1, 127.4, 127.3, 122.3, 53.4. HRMS (ESI) m/z: $[\mathrm{M}+\mathrm{H}]^{+}$calcd for $\mathrm{C}_{22} \mathrm{H}_{17} \mathrm{~N}_{2} \mathrm{O}_{3}$ 357.1234; found 357.1249. IR ( $\mathrm{KBr}$, v) $3072,2955,2925,2853,1734,1692,1590,1359,1247,1206,1178,1006,769,692$ $\mathrm{cm}^{-1}$. 
Methyl 3-(3-methoxyphenyl)-4-oxo-3,4-dihydroquinazoline-2carboxylate (5aj). Following the general procedure to synthesis the title compound. The pure production 5aj was isolated by flash chromatography column with $\mathrm{V}_{\mathrm{PE}}: \mathrm{V}_{\mathrm{EA}}(7: 1)$ as the eluent. Light yellow solid, $118 \mathrm{mg}$, 95\% yield; m.p. 80-81 ${ }^{\circ} \mathrm{C} .{ }^{1} \mathrm{H}$ NMR $\left(400 \mathrm{MHz}, \mathrm{CDCl}_{3}\right) \delta 8.33(\mathrm{~d}, J=8.0 \mathrm{~Hz}, 1 \mathrm{H}), 7.86-$ $7.78(\mathrm{~m}, 2 \mathrm{H}), 7.65-7.52(\mathrm{~m}, 1 \mathrm{H}), 7.38(\mathrm{t}, J=8.0 \mathrm{~Hz}, 1 \mathrm{H}), 7.05-6.97(\mathrm{~m}, 1 \mathrm{H}), 6.90(\mathrm{~d}, J$ $=7.2 \mathrm{~Hz}, 2 \mathrm{H}), 3.81(\mathrm{~s}, 3 \mathrm{H}), 3.66(\mathrm{~s}, 3 \mathrm{H}) .{ }^{13} \mathrm{C} \mathrm{NMR}\left(100 \mathrm{MHz}, \mathrm{CDCl}_{3}\right) \delta 161.3,160.8$, 160.3, 146.9, 146.4, 137.0, 135.1, 130.1, 128.7, 128.2, 127.4, 122.3, 120.1, 115.8, 113.7 , 55.6, 53.3. HRMS (ESI) $\mathrm{m} / \mathrm{z}$ : $[\mathrm{M}+\mathrm{H}]^{+}$calcd for $\mathrm{C}_{17} \mathrm{H}_{15} \mathrm{~N}_{2} \mathrm{O}_{4}$ 311.1026; found 311.1023. IR (KBr, v) 3077, 3020, 2958, 2938, 2838, 1752, 1690, 1602, 1471, 1462 , $1362,1231,1172,1043,937,808,779,698 \mathrm{~cm}^{-1}$.

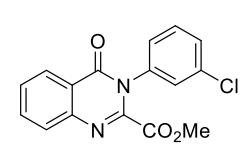

5 ak
Methyl

carboxylate (5ak). Following the general procedure to synthesis the title compound. The pure production 5ak was isolated by flash chromatography column with $\mathrm{V}_{\mathrm{PE}}: \mathrm{V}_{\mathrm{EA}}(8: 1)$ as the eluent. Yellow solid, $112 \mathrm{mg}, 89 \%$ yield; m.p. $134-135^{\circ} \mathrm{C} .{ }^{1} \mathrm{H}$ NMR $\left(400 \mathrm{MHz}, \mathrm{CDCl}_{3}\right) \delta 8.30(\mathrm{~d}, J=8.0 \mathrm{~Hz}, 1 \mathrm{H}), 7.85-$ $7.78(\mathrm{~m}, 2 \mathrm{H}), 7.62-7.54(\mathrm{~m}, 1 \mathrm{H}), 7.48-7.40(\mathrm{~m}, 2 \mathrm{H}), 7.37(\mathrm{~s}, 1 \mathrm{H}), 7.23(\mathrm{~d}, J=7.2 \mathrm{~Hz}$, 1H), 3.69 (s, 3H). ${ }^{13} \mathrm{C}$ NMR (100 MHz, $\left.\mathrm{CDCl}_{3}\right) \delta 161.1,160.7,146.3,146.1,137.2$, 135.2, 135.1, 130.4, 129.9, 129.0, 128.5, 128.4, 127.4, 126.3, 122.1, 53.5. HRMS (ESI) $\mathrm{m} / \mathrm{z}:[\mathrm{M}+\mathrm{H}]^{+}$calcd for $\mathrm{C}_{16} \mathrm{H}_{12} \mathrm{~N}_{2} \mathrm{O}_{3} \mathrm{Cl} 315.0531$; found 315.0531. IR ( $\left.\mathrm{KBr}, v\right)$ 3082, 2952, 1737, 1691, 1582, 1462, 1435, 1362, 1244, 1218, 1206, 1014, 877, $773 \mathrm{~cm}^{-1}$.

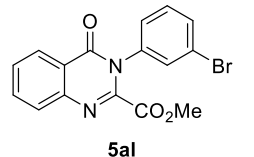

Methyl carboxylate (5al). Following the general procedure to synthesis the title compound. The pure production 5al was isolated by flash chromatography column with $\mathrm{V}_{\mathrm{PE}}$ : $\mathrm{V}_{\mathrm{EA}}(8: 1)$ as the eluent. Yellow solid, $132 \mathrm{mg}, 92 \%$ yield; m.p. $118-119^{\circ} \mathrm{C} .{ }^{1} \mathrm{H}$ NMR $\left(400 \mathrm{MHz}, \mathrm{CDCl}_{3}\right) \delta 8.29$ (d, $\left.J=8.0 \mathrm{~Hz}, 1 \mathrm{H}\right), 7.84-$ $7.78(\mathrm{~m}, 2 \mathrm{H}), 7.62-7.54(\mathrm{~m}, 2 \mathrm{H}), 7.51(\mathrm{~s}, 1 \mathrm{H}), 7.36(\mathrm{t}, J=8.0 \mathrm{~Hz}, 1 \mathrm{H}), 7.27(\mathrm{~d}, J=8.0$ $\mathrm{Hz}, 1 \mathrm{H}), 3.69$ (s, 3H). ${ }^{13} \mathrm{C} \mathrm{NMR}\left(100 \mathrm{MHz}, \mathrm{CDCl}_{3}\right) \delta 161.1,160.7,146.3,146.1,137.3$, 135.3, 132.8, 131.3, 130.6, 129.0, 128.4, 127.4, 126.8, 122.7, 122.1, 53.5. HRMS (ESI) $\mathrm{m} / \mathrm{z}:[\mathrm{M}+\mathrm{H}]^{+}$calcd for $\mathrm{C}_{16} \mathrm{H}_{12} \mathrm{~N}_{2} \mathrm{O}_{3} \mathrm{Br}$ 359.0026; found 359.0039. IR ( $\left.\mathrm{KBr}, v\right)$ 3083, 3066, 2956, 1740, 1697, 1576, 1462, 1360, 1246, 1207, 1013, 797, 777, $688 \mathrm{~cm}^{-1}$. 


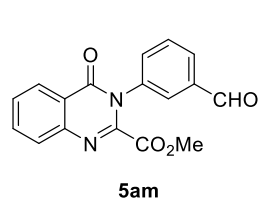

Methyl 3-(3-formylphenyl)-4-oxo-3,4-dihydroquinazoline-2carboxylate (5am). Following the general procedure to synthesis the title compound. The pure production 5am was isolated by flash chromatography column with $\mathrm{V}_{\mathrm{PE}}: \mathrm{V}_{\mathrm{EA}}(8: 1)$ as the eluent. Light yellow solid, $114 \mathrm{mg}$, 92\% yield; m.p. $158-160{ }^{\circ} \mathrm{C} .{ }^{1} \mathrm{H}$ NMR $\left(400 \mathrm{MHz}, \mathrm{CDCl}_{3}\right) \delta 10.03$ (s, 1H), 8.32 (d, $J=$ $8.0 \mathrm{~Hz}, 1 \mathrm{H}), 7.99$ (d, $J=7.6 \mathrm{~Hz}, 1 \mathrm{H}), 7.87-7.78(\mathrm{~m}, 3 \mathrm{H}), 7.69$ (t, $J=7.6 \mathrm{~Hz}, 1 \mathrm{H}), 7.63-$ 7.56 (m, 2H), 3.67 (s, 3H). $\left.{ }^{13} \mathrm{C} \mathrm{NMR} \mathrm{(100} \mathrm{MHz,} \mathrm{CDCl}_{3}\right) \delta 190.7,161.1,160.9,146.3$, 145.9, 137.6, 137.3, 135.4, 133.9, 130.6, 130.3, 129.1, 129.0, 128.5, 127.4, 122.1, 53.6. HRMS (ESI) m/z: $[\mathrm{M}+\mathrm{H}]^{+}$calcd for $\mathrm{C}_{17} \mathrm{H}_{13} \mathrm{~N}_{2} \mathrm{O}_{4}$ 309.0870; found 309.0879. IR (KBr, v) $3059,2960,2924,2849,2818,2744,1732,1690,1586,1466,1364,1256,1214$, $1022,879,778,689 \mathrm{~cm}^{-1}$.

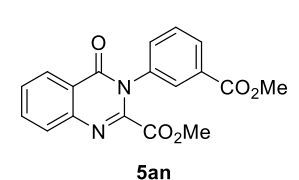

Methyl

3-(3-(methoxycarbonyl)phenyl)-4-oxo-3,4-

dihydroquinazoline-2-carboxylate (5an). Following the general procedure to synthesis the title compound. The pure production 5an was isolated by flash chromatography column with $\mathrm{V}_{\mathrm{PE}}: \mathrm{V}_{\mathrm{EA}}(5: 1)$ as the eluent. Yellow solid, $130 \mathrm{mg}$, $96 \%$ yield; m.p. $175-177^{\circ} \mathrm{C} .{ }^{1} \mathrm{H}$ NMR (400 MHz, $\left.\mathrm{CDCl}_{3}\right) \delta 8.34$ (d, $J=$ $8.0 \mathrm{~Hz}, 1 \mathrm{H}), 8.17(\mathrm{~d}, J=7.6 \mathrm{~Hz}, 1 \mathrm{H}), 8.02(\mathrm{~s}, 1 \mathrm{H}), 7.88-7.80(\mathrm{~m}, 2 \mathrm{H}), 7.63-7.53(\mathrm{~m}$, $3 \mathrm{H}), 3.92(\mathrm{~s}, 3 \mathrm{H}), 3.67$ (s, 3H). ${ }^{13} \mathrm{C} \mathrm{NMR}\left(100 \mathrm{MHz}, \mathrm{CDCl}_{3}\right) \delta 165.7,161.1,160.8$, $146.3,146.2$, 136.5, 135.2, 132.5, 131.7, 130.7, 129.5, 129.1, 128.9, 128.4, 127.3, 122.1, 53.4, 52.5. HRMS (ESI) $\mathrm{m} / \mathrm{z}$ : $[\mathrm{M}+\mathrm{H}]^{+}$calcd for $\mathrm{C}_{18} \mathrm{H}_{15} \mathrm{~N}_{2} \mathrm{O}_{5}$ 339.0975; found 339.0983. IR (KBr, v) 3068, 3013, 2954, 2850, 1748, 1698, 1600, 1465, 1436, 1374 , $1324,1282,1205,1121,1018,969,773,755,694 \mathrm{~cm}^{-1}$.

Methyl 4-oxo-3-(o-tolyl)-3,4-dihydroquinazoline-2-carboxylate (5ao).

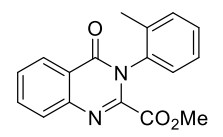

Following the general procedure to synthesis the title compound. The 5ao pure production 5ao was isolated by flash chromatography column with $\mathrm{V}_{\mathrm{PE}}: \mathrm{V}_{\mathrm{EA}}(8: 1)$ as the eluent. Yellow solid, $97 \mathrm{mg}, 82 \%$ yield; m.p. $113-115{ }^{\circ} \mathrm{C} .{ }^{1} \mathrm{H}$ NMR $\left(400 \mathrm{MHz}, \mathrm{CDCl}_{3}\right) \delta 8.36(\mathrm{~d}, J=8.0 \mathrm{~Hz}, 1 \mathrm{H}), 7.88-7.79(\mathrm{~m}, 2 \mathrm{H}), 7.63-7.55(\mathrm{~m}$, 1H), 7.41-7.34(m, 2H), 7.29 (t, $J=7.2 \mathrm{~Hz}, 1 \mathrm{H}), 7.15(\mathrm{~d}, J=7.6 \mathrm{~Hz}, 1 \mathrm{H}), 3.62(\mathrm{~s}, 3 \mathrm{H})$, $2.23(\mathrm{~s}, 3 \mathrm{H}) .{ }^{13} \mathrm{C}$ NMR $\left(100 \mathrm{MHz}, \mathrm{CDCl}_{3}\right) \delta 161.2,160.4,147.2,146.8,137.1,135.3$, 135.1, 131.2, 130.2, 128.7, 128.3, 128.0, 127.4, 127.1, 122.3, 53.3, 17.8. HRMS (ESI) $\mathrm{m} / \mathrm{z}:[\mathrm{M}+\mathrm{H}]^{+}$calcd for $\mathrm{C}_{17} \mathrm{H}_{15} \mathrm{~N}_{2} \mathrm{O}_{3} 295.1077$; found 295.1078. IR (KBr, v) 3080, 3047, 
3008, 2951, 2921, 1748, 1686, 1598, 1463, 1364, 1248, 1209, 1005, 928, 889, 771, 761, $701 \mathrm{~cm}^{-1}$.

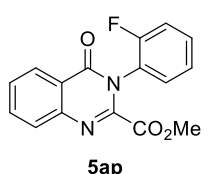

5 ap

\section{Methyl}

carboxylate (5ap). Following the general procedure to synthesis the title compound. The pure production 5ap was isolated by flash chromatography column with $\mathrm{V}_{\mathrm{PE}}: \mathrm{V}_{\mathrm{EA}}(7: 1)$ as the eluent. White solid, $111 \mathrm{mg}, 93 \%$ yield; m.p. $159-161{ }^{\circ} \mathrm{C} .{ }^{1} \mathrm{H}$ NMR $\left(400 \mathrm{MHz}, \mathrm{CDCl}_{3}\right) \delta 8.35$ (d, $\left.J=8.0 \mathrm{~Hz}, 1 \mathrm{H}\right), 7.90$ $7.82(\mathrm{~m}, 2 \mathrm{H}), 7.65-7.58(\mathrm{~m}, 1 \mathrm{H}), 7.55-7.45(\mathrm{~m}, 1 \mathrm{H}), 7.37-7.22(\mathrm{~m}, 3 \mathrm{H}), 3.72(\mathrm{~s}, 3 \mathrm{H})$. ${ }^{13} \mathrm{C} \mathrm{NMR}\left(100 \mathrm{MHz}, \mathrm{CDCl}_{3}\right) \delta 160.9,160.4,158.1\left(J_{C-F}=251 \mathrm{~Hz}\right), 146.3,146.0,135.3$, $131.7\left(J_{C-F}=8 \mathrm{~Hz}\right), 129.5,129.0,128.5,127.5,124.8\left(J_{C-F}=4 \mathrm{~Hz}\right), 124.2\left(J_{C-F}=14\right.$ $\mathrm{Hz}), 122.1,116.7\left(J_{C-F}=19 \mathrm{~Hz}\right), 53.5,{ }^{19} \mathrm{~F}$ NMR $\left(376 \mathrm{MHz}, \mathrm{CDCl}_{3}\right) \delta-119.8$. HRMS (ESI) $\mathrm{m} / \mathrm{z}:[\mathrm{M}+\mathrm{H}]^{+}$calcd for $\mathrm{C}_{16} \mathrm{H}_{12} \mathrm{~N}_{2} \mathrm{O}_{3} \mathrm{~F}$ 299.0826; found 299.0835. IR (KBr, v) 3078, 2956, 2848, 1749, 1710, 1615, 1602, 1500, 1362, 1251, 1203, 1009, 891, 811, $771,760,693 \mathrm{~cm}^{-1}$.

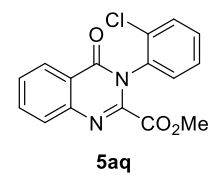

\section{Methyl}

3-(2-chlorophenyl)-4-oxo-3,4-dihydroquinazoline-2carboxylate (5aq). Following the general procedure to synthesis the title compound. The pure production 5aq was isolated by flash chromatography column with $\mathrm{V}_{\mathrm{PE}}: \mathrm{V}_{\mathrm{EA}}(8: 1)$ as the eluent. White solid, $111 \mathrm{mg}, 88 \%$ yield; m.p. $153-154{ }^{\circ} \mathrm{C} .{ }^{1} \mathrm{H} \mathrm{NMR}\left(400 \mathrm{MHz}, \mathrm{CDCl}_{3}\right) \delta 8.36$ (d, $\left.J=8.0 \mathrm{~Hz}, 1 \mathrm{H}\right), 7.90$ $7.81(\mathrm{~m}, 2 \mathrm{H}), 7.64-7.54(\mathrm{~m}, 2 \mathrm{H}), 7.47-7.36(\mathrm{~m}, 3 \mathrm{H}), 3.71$ (s, 3H). ${ }^{13} \mathrm{C}$ NMR (100 MHz, $\left.\mathrm{CDCl}_{3}\right) \delta 160.8,160.4,146.3,145.7,135.3,134.3,133.0,131.1,130.4,130.1,129.1$, 128.5, 127.8, 127.5, 122.3, 53.6. HRMS (ESI) m/z: $[\mathrm{M}+\mathrm{H}]^{+}$calcd for $\mathrm{C}_{16} \mathrm{H}_{12} \mathrm{~N}_{2} \mathrm{O}_{3} \mathrm{Cl}$ 315.0531; found 315.0547. IR (KBr, v) 3080, 3023, 2953, 2848, 1749, 1694, 1600 , $1463,1362,1244,1208,1004,928,770,698 \mathrm{~cm}^{-1}$.

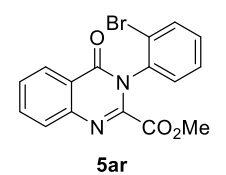

Methyl

3-(2-bromophenyl)-4-oxo-3,4-dihydroquinazoline-2carboxylate (5ar). Following the general procedure to synthesis the title compound. The pure production 5ar was isolated by flash chromatography column with $\mathrm{V}_{\mathrm{PE}}: \mathrm{V}_{\mathrm{EA}}(8: 1)$ as the eluent. Brown solid, $110 \mathrm{mg}, 77 \%$ yield; m.p. $140-142{ }^{\circ} \mathrm{C} . \quad{ }^{1} \mathrm{H} \mathrm{NMR}\left(400 \mathrm{MHz}, \mathrm{CDCl}_{3}\right) \delta 8.37$ (d, J=8.0 Hz, 1H), 7.91$7.82(\mathrm{~m}, 2 \mathrm{H}), 7.74(\mathrm{~d}, J=8.0 \mathrm{~Hz}, 1 \mathrm{H}), 7.62(\mathrm{t}, J=7.2 \mathrm{~Hz}, 1 \mathrm{H}), 7.48(\mathrm{t}, J=7.2 \mathrm{~Hz}, 1 \mathrm{H})$, 7.43-7.33 (m, 2H), $3.73(\mathrm{~s}, 3 \mathrm{H}) .{ }^{13} \mathrm{C}$ NMR (100 MHz, $\left.\mathrm{CDCl}_{3}\right) \delta 160.8,160.3,146.3$, $145.5,135.9,135.3,133.6,131.2,130.2$, 129.0, 128.5, 128.5, 127.5, 123.0, 122.3, 53.5. 
HRMS (ESI) $\mathrm{m} / \mathrm{z}$ : $[\mathrm{M}+\mathrm{H}]^{+}$calcd for $\mathrm{C}_{16} \mathrm{H}_{12} \mathrm{~N}_{2} \mathrm{O}_{3} \mathrm{Br}$ 359.0026; found 359.0030. IR $(\mathrm{KBr}, v)$ 3075, 3017, 2953, 2848, 1751, 1693, 1600, 1462, 1363, 1243, 1207, 1003, 928 , $887,768,697 \mathrm{~cm}^{-1}$.

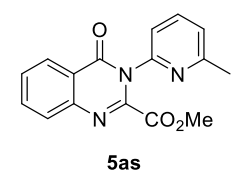

Methyl 3-(6-methylpyridin-2-yl)-4-oxo-3,4-dihydroquinazoline-2carboxylate (5as). Following the general procedure to synthesis the title compound. The pure production 5as was isolated by flash chromatography column with $\mathrm{V}_{\mathrm{PE}}: \mathrm{V}_{\mathrm{EA}}(8: 1)$ as the eluent. White solid, $116 \mathrm{mg}, 98 \%$ yield; m.p. $148-149{ }^{\circ} \mathrm{C} .{ }^{1} \mathrm{H}$ NMR $\left(400 \mathrm{MHz}, \mathrm{CDCl}_{3}\right) \delta 8.34$ (d, $\left.J=8.0 \mathrm{~Hz}, 1 \mathrm{H}\right), 7.88$ $7.76(\mathrm{~m}, 3 \mathrm{H}), 7.58(\mathrm{t}, J=8.0 \mathrm{~Hz}, 2 \mathrm{H}), 7.21(\mathrm{~d}, J=7.2 \mathrm{~Hz}, 1 \mathrm{H}), 3.78(\mathrm{~s}, 3 \mathrm{H}), 2.51(\mathrm{~s}$, $3 \mathrm{H}) .{ }^{13} \mathrm{C} \mathrm{NMR}\left(100 \mathrm{MHz}, \mathrm{CDCl}_{3}\right) \delta 162.1,160.6,158.1,148.9,146.1,145.7,138.3$, 135.2, 129.0, 128.5, 127.4, 123.5, 122.2, 119.9, 53.4, 24.0. HRMS (ESI) m/z: $[\mathrm{M}+\mathrm{H}]^{+}$ calcd for $\mathrm{C}_{16} \mathrm{H}_{14} \mathrm{~N}_{3} \mathrm{O}_{3}$ 296.1030; found 296.1010. IR (KBr, v) 3080, 2952, 2849, 1742 , $1702,1599,1459,1357,1203,1026,944,877,821,769,693 \mathrm{~cm}^{-1}$.

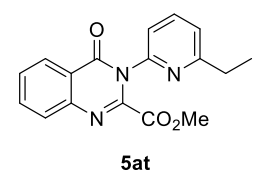

Methyl 3-(6-ethylpyridin-2-yl)-4-oxo-3,4-dihydroquinazoline-2carboxylate (5at). Following the general procedure to synthesis the title compound. The pure production 5at was isolated by flash chromatography column with $\mathrm{V}_{\mathrm{PE}}: \mathrm{V}_{\mathrm{EA}}(8: 1)$ as the eluent. White solid, $120 \mathrm{mg}, 97 \%$ yield; m.p. $123-124{ }^{\circ} \mathrm{C} .{ }^{1} \mathrm{H}$ NMR $\left(400 \mathrm{MHz}, \mathrm{CDCl}_{3}\right) \delta 8.35(\mathrm{~d}, J=8.0 \mathrm{~Hz}, 1 \mathrm{H}), 7.88$ $7.78(\mathrm{~m}, 3 \mathrm{H}), 7.59$ (t, $J=7.6 \mathrm{~Hz}, 2 \mathrm{H}), 7.21(\mathrm{~d}, J=7.6 \mathrm{~Hz}, 1 \mathrm{H}), 3.76$ (s, 3H), 2.77 (q, $J$ $=7.6 \mathrm{~Hz}, 2 \mathrm{H}), 1.26(\mathrm{t}, J=7.6 \mathrm{~Hz}, 3 \mathrm{H}) .{ }^{13} \mathrm{C} \mathrm{NMR}\left(100 \mathrm{MHz}, \mathrm{CDCl}_{3}\right) \delta 163.2,162.1$, 160.6, 149.0, 146.1, 145.9, 138.3, 135.2, 129.0, 128.5, 127.4, 122.3, 122.2, 120.0, 53.4, 30.8, 13.5. HRMS (ESI) $\mathrm{m} / \mathrm{z}:[\mathrm{M}+\mathrm{H}]^{+}$calcd for $\mathrm{C}_{17} \mathrm{H}_{16} \mathrm{~N}_{3} \mathrm{O}_{3}$ 310.1186; found 310.1179. IR (KBr, v) 2980, 2952, 2923, 1743, 1702, 1598, 1463, 1359, 1202, 1178, $1022,997,874,825,768,756,691 \mathrm{~cm}^{-1}$.

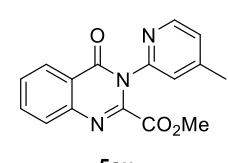

Methyl 3-(4-methylpyridin-2-yl)-4-oxo-3,4-dihydroquinazoline-2carboxylate (5au). Following the general procedure to synthesis the title compound. The pure production 5au was isolated by flash chromatography column with $\mathrm{V}_{\mathrm{PE}}: \mathrm{V}_{\mathrm{EA}}(8: 1)$ as the eluent. Light yellow solid, $116 \mathrm{mg}$, 98\% yield; m.p. $171-172{ }^{\circ} \mathrm{C} .{ }^{1} \mathrm{H}$ NMR (400 MHz, $\left.\mathrm{CDCl}_{3}\right) \delta$ 8.39-8.27 (m, 2H), 7.917.77 (m, 2H), 7.63-7.51 (m, 2H), 7.17 (s, 1H), 3.78 (s, 3H), 2.47 (s, 3H). ${ }^{13} \mathrm{C} \mathrm{NMR}(100$ $\left.\mathrm{MHz}, \mathrm{CDCl}_{3}\right) \delta 162.0,160.6,149.8,149.8,148.3,146.1,145.6,135.2,129.0,128.6$, 127.4, 125.3, 123.7, 122.2, 53.6, 21.3. HRMS (ESI) m/z: $[\mathrm{M}+\mathrm{H}]^{+}$calcd for 
$\mathrm{C}_{16} \mathrm{H}_{14} \mathrm{~N}_{3} \mathrm{O}_{3}$ 296.1030; found 296.1036. IR (KBr, v) 3085, 3009, 2958, 2920, 2858, $1740,1698,1600,1411,1365,1258,1209,1026,943,880,834,772,695 \mathrm{~cm}^{-1}$.

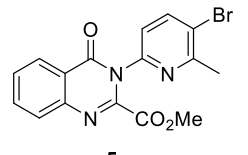

5 av 3-(5-bromo-6-methylpyridin-2-yl)-4-oxo-3,4dihydroquinazoline-2-carboxylate (5av). Following the general procedure to synthesis the title compound. The pure production 5av was isolated by flash chromatography column with $\mathrm{V}_{\mathrm{PE}}: \mathrm{V}_{\mathrm{EA}}(8: 1)$ as the eluent. Light yellow solid, $147 \mathrm{mg}, 98 \%$ yield; m.p. $168-170{ }^{\circ} \mathrm{C} .{ }^{1} \mathrm{H}$ NMR (400 MHz, $\left.\mathrm{CDCl}_{3}\right) \delta 8.33$ $(\mathrm{d}, J=8.0 \mathrm{~Hz}, 1 \mathrm{H}), 7.99$ (d, $J=8.4 \mathrm{~Hz}, 1 \mathrm{H}), 7.89-7.78(\mathrm{~m}, 2 \mathrm{H}), 7.59$ (t, $J=7.6 \mathrm{~Hz}$, $1 \mathrm{H}), 7.49(\mathrm{~d}, J=8.4 \mathrm{~Hz}, 1 \mathrm{H}), 3.82(\mathrm{~s}, 3 \mathrm{H}), 2.59$ (s, 3H). ${ }^{13} \mathrm{C} \mathrm{NMR}\left(100 \mathrm{MHz}, \mathrm{CDCl}_{3}\right)$ $\delta 162.0,160.4,156.9,147.7,146.0,145.3,141.7,135.4,129.2,128.6,127.5,122.0$, 121.5, 121.2, 53.5, 24.5. HRMS (ESI) m/z: $[\mathrm{M}+\mathrm{H}]^{+}$calcd for $\mathrm{C}_{16} \mathrm{H}_{13} \mathrm{~N}_{3} \mathrm{O}_{3} \mathrm{Br}$ 374.0135; found 374.0139. IR (KBr, v) 3067, 2962, 1737, 1698, 1600, 1432, 1354, 1251, 1210 , $1033,942,880,842,772,699 \mathrm{~cm}^{-1}$.

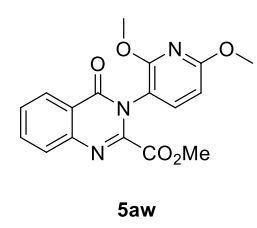

Methyl

3-(2,6-dimethoxypyridin-3-yl)-4-oxo-3,4dihydroquinazoline-2-carboxylate (5aw). Following the general procedure to synthesis the title compound. The pure production 5aw was isolated by flash chromatography column with $\mathrm{V}_{\mathrm{PE}}: \mathrm{V}_{\mathrm{EA}}(8: 1)$ as the eluent. Light yellow solid, $119 \mathrm{mg}$, 87\% yield; m.p. $146-147{ }^{\circ} \mathrm{C} .{ }^{1} \mathrm{H}$ NMR (400 MHz, $\left.\mathrm{CDCl}_{3}\right) \delta 8.32(\mathrm{~d}, J=8.0 \mathrm{~Hz}, 1 \mathrm{H}), 7.86-7.76(\mathrm{~m}, 2 \mathrm{H}), 7.60-7.53(\mathrm{~m}, 1 \mathrm{H}), 7.50(\mathrm{~d}, J=$ $8.4 \mathrm{~Hz}, 1 \mathrm{H}), 6.42(\mathrm{~d}, J=8.4 \mathrm{~Hz}, 1 \mathrm{H}), 3.95(\mathrm{~s}, 3 \mathrm{H}), 3.89(\mathrm{~s}, 3 \mathrm{H}), 3.75(\mathrm{~s}, 3 \mathrm{H}) .{ }^{13} \mathrm{C}$ NMR $\left(100 \mathrm{MHz}, \mathrm{CDCl}_{3}\right) \delta 163.5,161.3,160.9,157.8,147.1,146.4,140.6,135.0,128.6$, 128.3, 127.4, 122.3, 111.3, 101.8, 54.0, 53.9, 53.4. HRMS (ESI) m/z: $[\mathrm{M}+\mathrm{H}]^{+}$calcd for $\mathrm{C}_{17} \mathrm{H}_{16} \mathrm{~N}_{3} \mathrm{O}_{5}$ 342.1084; found 342.1074. IR ( $\left.\mathrm{KBr}, v\right)$ 3023, 2984, 2952, 1744, 1702, $1609,1485,1335,1253,1210,1014,887,812,781,768,702 \mathrm{~cm}^{-1}$.

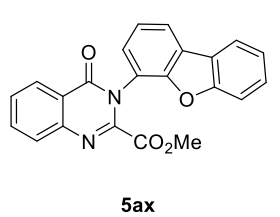

Methyl

3-(dibenzo $[b, d]$ furan-4-yl)-4-oxo-3,4dihydroquinazoline-2-carboxylate (5ax). Following the general procedure to synthesis the title compound. The pure production 5ax was isolated by flash chromatography column with $\mathrm{V}_{\mathrm{PE}}$ : $\mathrm{V}_{\mathrm{EA}}(8: 1)$ as the eluent. White solid, $142 \mathrm{mg}$, 96\% yield; m.p. $232-233{ }^{\circ} \mathrm{C} .{ }^{1} \mathrm{H}$ NMR (400 MHz, $\left.\mathrm{CDCl}_{3}\right) \delta 8.41(\mathrm{~d}, J=7.6 \mathrm{~Hz}, 1 \mathrm{H}), 8.07(\mathrm{~d}, J=7.2 \mathrm{~Hz}, 1 \mathrm{H}), 7.98(\mathrm{~d}, J=7.6 \mathrm{~Hz}, 1 \mathrm{H})$, 7.94-7.84 (m, 2H), $7.63(\mathrm{t}, J=7.6 \mathrm{~Hz}, 1 \mathrm{H}), 7.54(\mathrm{~d}, J=8.0 \mathrm{~Hz}, 1 \mathrm{H}), 7.49-7.35(\mathrm{~m}, 4 \mathrm{H})$, $3.56(\mathrm{~s}, 3 \mathrm{H}) .{ }^{13} \mathrm{C} \mathrm{NMR}\left(100 \mathrm{MHz}, \mathrm{CDCl}_{3}\right) \delta 161.1,160.5,156.5,151.5,146.5,146.5$, 
$135.2,129.0,128.5,128.0,127.6,126.3,126.2,123.8,123.5,123.3,122.3,121.1,120.9$, 112.2, 53.4. HRMS (ESI) $\mathrm{m} / \mathrm{z}$ : $[\mathrm{M}+\mathrm{H}]^{+}$calcd for $\mathrm{C}_{22} \mathrm{H}_{15} \mathrm{~N}_{2} \mathrm{O}_{4}$ 371.1026; found 371.1027. IR (KBr, v) 3064, 3038, 2950, 2923, 2845, 1748, 1686, 1607, 1435, 1363 , $1250,1194,1006,939,838,775,753,697 \mathrm{~cm}^{-1}$.

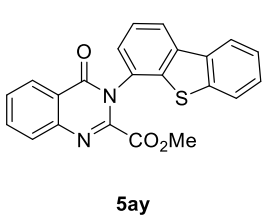

Methyl

3-(dibenzo $[b, d]$ thiophen-4-yl)-4-oxo-3,4dihydroquinazoline-2-carboxylate (5ay). Following the general procedure to synthesis the title compound. The pure production 5ay was isolated by flash chromatography column with $\mathrm{V}_{\mathrm{PE}}: \mathrm{V}_{\mathrm{EA}}(8: 1)$ as the eluent. White solid, $127 \mathrm{mg}, 82 \%$ yield; m.p. $264-265{ }^{\circ} \mathrm{C} .{ }^{1} \mathrm{H}$ NMR $(400 \mathrm{MHz}$, $\left.\mathrm{CDCl}_{3}\right) \delta 8.41(\mathrm{~d}, J=8.0 \mathrm{~Hz}, 1 \mathrm{H}), 8.27(\mathrm{~d}, J=8.0 \mathrm{~Hz}, 1 \mathrm{H}), 8.19(\mathrm{~d}, J=6.8 \mathrm{~Hz}, 1 \mathrm{H})$, 7.94-7.86 (m, 2H), $7.80(\mathrm{~d}, J=7.2 \mathrm{~Hz}, 1 \mathrm{H}), 7.65(\mathrm{t}, J=7.6 \mathrm{~Hz}, 1 \mathrm{H}), 7.59$ (t, $J=7.6 \mathrm{~Hz}$, 1H), 7.52-7.46 (m, 2H), 7.40 (d, $J=7.6 \mathrm{~Hz}, 1 \mathrm{H}), 3.57$ (s, 3H). ${ }^{13} \mathrm{C}$ NMR (100 MHz, $\left.\mathrm{CDCl}_{3}\right) \delta 161.0,160.1,146.5,146.4,139.4,139.0,137.8,135.4,131.4,129.1,128.6$, 127.7, 127.6, 126.3, 125.4, 124.9, 123.1, 122.9, 122.3, 122.2, 53.5. HRMS (ESI) m/z: $[\mathrm{M}+\mathrm{H}]^{+}$calcd for $\mathrm{C}_{22} \mathrm{H}_{15} \mathrm{~N}_{2} \mathrm{O}_{3} \mathrm{~S}$ 387.0798; found 387.0802. IR ( $\left.\mathrm{KBr}, v\right)$ 3061, 2953, 2924, 2851, 1756, 1684, 1610, 1442, 1353, 1250, 1203, 1001, 937, 767, 756, $692 \mathrm{~cm}^{-1}$.

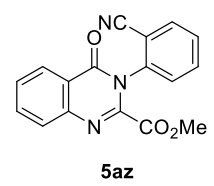

Methyl

3-(2-cyanophenyl)-4-oxo-3,4-dihydroquinazoline-2-

carboxylate (5az). Following the general procedure to synthesis the title compound. The pure production 5az was isolated by flash chromatography column with $\mathrm{V}_{\mathrm{PE}}: \mathrm{V}_{\mathrm{DCM}}: \mathrm{V}_{\mathrm{EA}}(5: 5: 1)$ as the eluent. Light yellow solid, $104 \mathrm{mg}, 85 \%$ yield; m.p. $169-170{ }^{\circ} \mathrm{C} .{ }^{1} \mathrm{H}$ NMR $\left(400 \mathrm{MHz}, \mathrm{CDCl}_{3}\right) \delta 8.36$ (d, $J=8.4$ $\mathrm{Hz}, 1 \mathrm{H}), 7.93-7.82(\mathrm{~m}, 3 \mathrm{H}), 7.74(\mathrm{t}, J=8.0 \mathrm{~Hz}, 1 \mathrm{H}), 7.68-7.57(\mathrm{~m}, 2 \mathrm{H}), 7.39$ (d, $J=$ $8.0 \mathrm{~Hz}, 1 \mathrm{H}), 3.74(\mathrm{~s}, 3 \mathrm{H}) .{ }^{13} \mathrm{C} \mathrm{NMR}\left(100 \mathrm{MHz}, \mathrm{CDCl}_{3}\right) \delta 160.7,160.6,146.1,144.4$, 139.4, 135.6, 133.9, 133.6, 130.1, 129.6, 129.1, 128.8, 127.6, 122.0, 115.3, 113.8, 53.8. HRMS (ESI) m/z: [M + Na] $]^{+}$calcd for $\mathrm{C}_{17} \mathrm{H}_{11} \mathrm{~N}_{3} \mathrm{O}_{3} \mathrm{Na}$ 328.0693; found 328.0707. IR $(\mathrm{KBr}, v)$ 3108, 3039, 2953, 2233, 1740, 1693, 1598, 1249, 1209, $777 \mathrm{~cm}^{-1}$.

\section{Ethyl 6-oxo-1-phenyl-1,6-dihydropyrimidine-2-carboxylate (5sa).}

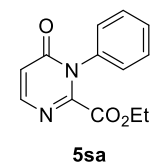

Following the general procedure to synthesis the title compound. The pure ${ }_{5 s a}$ production $5 \mathbf{s a}$ was isolated by flash chromatography column with $\mathrm{V}_{\mathrm{PE}}: \mathrm{V}_{\mathrm{EA}}$ (8:1) as the eluent. White solid, $77 \mathrm{mg}, 79 \%$ yield; m.p. 102-104 ${ }^{\circ} \mathrm{C} .{ }^{1} \mathrm{H}$ NMR (400 $\left.\mathrm{MHz} \mathrm{CDCl}_{3}\right) \delta 7.95(\mathrm{~d}, J=6.8 \mathrm{~Hz}, 1 \mathrm{H}), 7.53-7.44(\mathrm{~m}, 3 \mathrm{H}), 7.33-7.27(\mathrm{~m}, 2 \mathrm{H}), 6.61$ $(\mathrm{d}, J=6.4 \mathrm{~Hz}, 1 \mathrm{H}), 4.06(\mathrm{q}, J=7.2 \mathrm{~Hz}, 2 \mathrm{H}), 0.99(\mathrm{t}, J=7.2 \mathrm{~Hz}, 3 \mathrm{H}) .{ }^{13} \mathrm{C}$ NMR $(100$ 
$\left.\mathrm{MHz}, \mathrm{CDCl}_{3}\right) \delta 160.4,160.4,152.4,152.1,135.5,130.0,129.6,127.8,117.6,63.0,13.6$ HRMS (ESI) m/z: $[\mathrm{M}+\mathrm{H}]^{+}$calcd for $\mathrm{C}_{13} \mathrm{H}_{13} \mathrm{~N}_{2} \mathrm{O}_{3}$ 245.0921; found 245.0937. IR (KBr, v) $3072,3057,2987,2926,1747,1698,1530,1381,1289,1228,1192,1065,1014,843$, $775,759,698,579 \mathrm{~cm}^{-1}$.

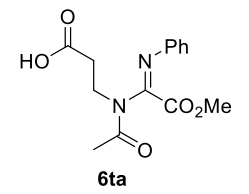

\section{(E)-3-(N-(2-methoxy-2-oxo-1-} (phenylimino)ethyl)acetamido)propanoic acid (6ta). Following the general procedure to synthesis the title compound. The pure production 6ta was isolated by flash chromatography column with $\mathrm{V}_{\mathrm{DCM}}: \mathrm{V}_{\mathrm{MeOH}}(15: 1)$ as the eluent. White solid, $102 \mathrm{mg}, 88 \%$ yield; m.p. 50-52 ${ }^{\circ} \mathrm{C} .{ }^{1} \mathrm{H}$ NMR $\left(400 \mathrm{MHz}, \mathrm{CDCl}_{3}\right) \delta$ $7.28(\mathrm{t}, J=7.2 \mathrm{~Hz}, 2 \mathrm{H}), 7.09(\mathrm{t}, J=7.2 \mathrm{~Hz}, 1 \mathrm{H}), 6.82(\mathrm{~d}, J=7.6 \mathrm{~Hz}, 2 \mathrm{H}), 4.14(\mathrm{t}, J=$ $7.2 \mathrm{~Hz}, 2 \mathrm{H}), 3.51(\mathrm{~s}, 3 \mathrm{H}), 2.88(\mathrm{t}, J=7.2 \mathrm{~Hz}, 2 \mathrm{H}), 2.38$ (s, 3H). ${ }^{13} \mathrm{C}$ NMR (100 MHz, $\left.\mathrm{CDCl}_{3}\right) \delta 176.3,172.2,161.2,148.1,146.6,128.8,124.6,120.1,52.2,41.8,32.7,23.2$. HRMS (ESI) m/z: [M + Na] $]^{+}$calcd for $\mathrm{C}_{14} \mathrm{H}_{16} \mathrm{~N}_{2} \mathrm{O}_{5} \mathrm{Na} 315.0951$; found 315.0958. IR $(\mathrm{KBr}, v)$ 3448, 3061, 2956, 1718, 1690, 1656, 1395, 1216, 1064, 1012, 770, $697 \mathrm{~cm}^{-1}$.

\section{Gram-Scale Synthesis of 5ar.}

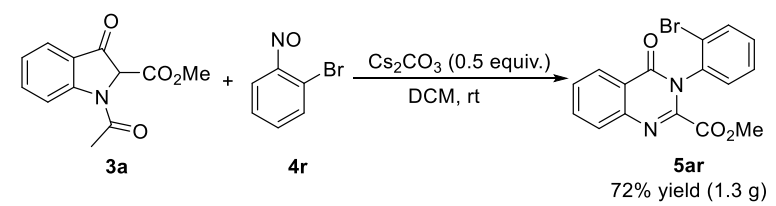

A solution of 3a $(1.16 \mathrm{~g}, 5.0 \mathrm{mmol}), o$-bromonitrosobenzene $4 \mathbf{r}(1.11 \mathrm{~g}, 6.0 \mathrm{mmol})$ and $\mathrm{Cs}_{2} \mathrm{CO}_{3}(0.814 \mathrm{~g}, 2.5 \mathrm{mmol}$.) in DCM $(12 \mathrm{~mL})$ were stirred at room temperature for 10 days. The reaction mixture concentrated under reduced pressure. The crude product was purified by column chromatography on silica gel with $\mathrm{V}_{\mathrm{PE}}: \mathrm{V}_{\mathrm{EA}}(8: 1)$ as the eluent to afford the product $\mathbf{5}$ ar in $72 \%$ yield.

\section{General Procedure for the Synthesis of 8ar and 9ar.}

6.1 General procedure for the synthesis of $\mathbf{8 a r} .^{[6]}$

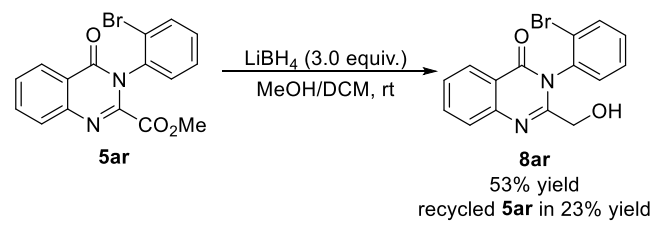


The compound 5 ar $(1.39 \mathrm{~g}, 3.9 \mathrm{mmol})$ and $\mathrm{V}_{\mathrm{MeOH}}: \mathrm{V}_{\mathrm{DCM}}(25 \mathrm{~mL}: 25 \mathrm{~mL})$ was added into a round flask. Then $\mathrm{LiBH}_{4}(0.25 \mathrm{~g}, 3.0$ equiv.) was added into the reaction mixture and stirred at room temperature for $17 \mathrm{~h}$. Then slowly added $5 \% \mathrm{HCl}$ aqueous to the reaction mixture to adjust the $\mathrm{PH}$ of the reaction mixture to 4-3. The reaction mixture concentrated under reduced pressure. The mixture was extracted with EA $(40 \mathrm{~mL} \times 3)$. The collected organic layers were dried over $\mathrm{Na}_{2} \mathrm{SO}_{4}$, filtered and concentrated under reduced pressure. The crude product was purified by column chromatography on silica gel with $\mathrm{V}_{\mathrm{PE}}: \mathrm{V}_{\mathrm{EA}}=3: 1$ as the eluent to afford the product 8ar in 53\% yield and recycled the raw matrial in $23 \%$ yield. 3-(2-bromophenyl)-2(hydroxymethyl)quinazolin-4(3H)-one (8ar). White solid, $682 \mathrm{mg}$, 53\% yield; m.p. 118-119 ${ }^{\circ} \mathrm{C} .{ }^{1} \mathrm{H}$ NMR $\left(400 \mathrm{MHz}, \mathrm{CDCl}_{3}\right) \delta 8.32(\mathrm{~d}, J=8.0 \mathrm{~Hz}, 1 \mathrm{H}), 7.88-7.73(\mathrm{~m}, 3 \mathrm{H})$, $7.53(\mathrm{t}, J=7.2 \mathrm{~Hz}, 2 \mathrm{H}), 7.43(\mathrm{t}, J=7.2 \mathrm{~Hz}, 1 \mathrm{H}), 7.34(\mathrm{~d}, J=7.6 \mathrm{~Hz}, 1 \mathrm{H}), 4.25(\mathrm{~s}, 1 \mathrm{H})$, $4.09(\mathrm{dd}, J=34.4,16.4 \mathrm{~Hz}, 2 \mathrm{H}) .{ }^{13} \mathrm{C} \mathrm{NMR}\left(100 \mathrm{MHz}, \mathrm{CDCl}_{3}\right) \delta 161.0,154.7,146.4$, 135.2, 134.3, 134.1, 131.7, 130.0, 129.3, 127.6, 127.4, 127.0, 123.0, 121.0, 60.5. HRMS (ESI) $\mathrm{m} / \mathrm{z}:[\mathrm{M}+\mathrm{H}]^{+}$calcd for $\mathrm{C}_{15} \mathrm{H}_{12} \mathrm{BrN}_{2} \mathrm{O}_{2}$ 331.0077; found 331.0088. IR (KBr, v) $3423,3076,1689,1610,1473,1342,1321,1295,1107,1001,768,695, \mathrm{~cm}^{-1}$.

6.2 General procedure for the synthesis of 9ar. ${ }^{[7]}$

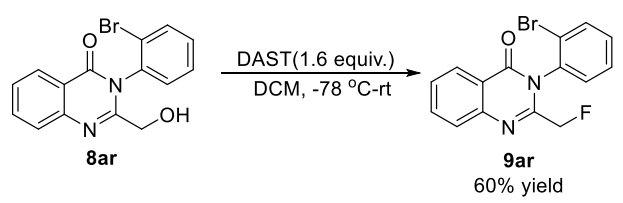

The compound $8 \mathbf{a r}(0.06 \mathrm{~g}, 0.2 \mathrm{mmol})$ was added in a dried round flask. The flask was degassed via vacuum and backfilled with argon gas. The purge and backfilled process was repeated three times. Then dried DCM $(2.0 \mathrm{~mL})$ was added to the flask, and the reaction mixture was stirred at $-78{ }^{\circ} \mathrm{C}$. DAST $(0.05 \mathrm{~g}, 0.32 \mathrm{mmol})$ was resolved with dried DCM $(1.0 \mathrm{~mL})$ and the DCM solution of DAST was added into the raction mixture slowly. The reaction mixture was stirred at $-78^{\circ} \mathrm{C}$ for $1 \mathrm{~h}$. Then adjusted the reaction tepmrature to $\mathrm{rt}$ stirred for $9 \mathrm{~h}$. Ice water was added to the reaction mixture to quench the reaction. Then saturated $\mathrm{NaHCO}_{3}$ solution and DCM was added the reaction. the resulting mixture was extracted with $\mathrm{DCM}(20 \mathrm{~mL} \times 3)$. The collected organic layers were dried over $\mathrm{Na}_{2} \mathrm{SO}_{4}$, filtered and concentrated under reduced pressure. The crude product was purified by column chromatography on silica gel with $\mathrm{V}_{\mathrm{PE}}$ : $\mathrm{V}_{\mathrm{EA}}$ (3:1) as the eluent to afford the product 9ar as a white solid in 60\% yield. 3-(2- 
bromophenyl)-2-(fluoromethyl)quinazolin-4(3H)-one (9ar). m.p. 127-128 ${ }^{\circ} \mathrm{C}$., 40 mg, 60\% yield. ${ }^{1} \mathrm{H}$ NMR (400 MHz, $\left.\mathrm{CDCl}_{3}\right) \delta 8.33(\mathrm{~d}, J=8.0 \mathrm{~Hz}, 1 \mathrm{H}), 7.84(\mathrm{~d}, J=3.6$ $\mathrm{Hz}, 2 \mathrm{H}), 7.79(\mathrm{~d}, J=8.0 \mathrm{~Hz}, 1 \mathrm{H}), 7.60-7.54(\mathrm{~m}, 1 \mathrm{H}), 7.52(\mathrm{~d}, J=8.4 \mathrm{~Hz}, 1 \mathrm{H}), 7.43$ (t, $J=7.6 \mathrm{~Hz}, 2 \mathrm{H}), 5.00(\mathrm{ddd}, J=46.6,34.3,12.1 \mathrm{~Hz}, 2 \mathrm{H}) .{ }^{13} \mathrm{C} \mathrm{NMR}\left(100 \mathrm{MHz}, \mathrm{CDCl}_{3}\right)$ $\delta 161.1,149.7,147.0,135.2,135.0,133.9,131.6,130.9,129.1,128.2,128.1,127.5$, 123.0, 121.5, $81.2\left(J_{C-F}=176.4 \mathrm{~Hz}\right) .{ }^{19} \mathrm{~F} \mathrm{NMR}\left(376 \mathrm{MHz}, \mathrm{CDCl}_{3}\right) \delta$-219.7. HRMS (ESI) m/z: $[\mathrm{M}+\mathrm{H}]^{+}$calcd for $\mathrm{C}_{15} \mathrm{H}_{11} \mathrm{BrFN}_{2} \mathrm{O} 333.0033$; found 333.0041. IR (KBr, $v$ ) 3443, 3065, 2927, 1694, 1608, 1472, 1464, 1350, 1285, 1111, 1028, 774, $696 \mathrm{~cm}^{-1}$.

\section{The Detected and Separated of the Intermediates.}

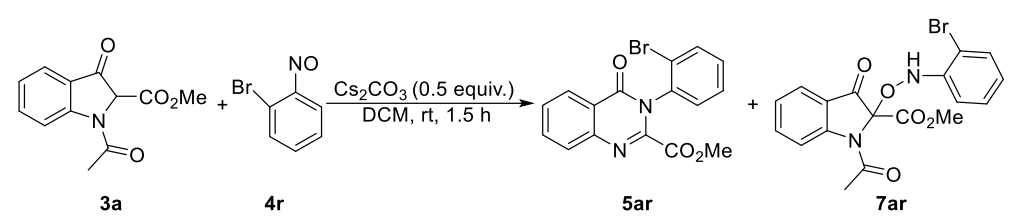

A solution of 3a $(0.4 \mathrm{mmol}), o$-bromonitrosobenzene $4 \mathbf{r}(0.48 \mathrm{mmol})$ and $\mathrm{Cs}_{2} \mathrm{CO}_{3}$ $(0.065 \mathrm{~g}, 0.2 \mathrm{mmol}$.) in DCM $(2 \mathrm{~mL})$ were stirred at room temperature for $1.5 \mathrm{~h}$. The reaction detected by TLC. The reaction mixture concentrated under reduced pressure. The crude product was purified by column chromatography on silica gel with $\mathrm{V}_{\mathrm{PE}}$ : $\mathrm{V}_{\mathrm{EA}}$ (5:1) as the eluent to afford the product 7ar as a white solid. Methyl 1-acetyl-2-(()bromophenyl)amino)oxy)-3-oxoindoline-2-carboxylate (7ar). m.p. $168-169{ }^{\circ} \mathrm{C} .{ }^{1} \mathrm{H}$ NMR (400 MHz, $\left.\mathrm{CDCl}_{3}\right) \delta 8.58(\mathrm{~s}, 1 \mathrm{H}), 7.77-7.61(\mathrm{~m}, 4 \mathrm{H}), 7.42-7.29(\mathrm{~m}, 2 \mathrm{H}), 7.23(\mathrm{t}$, $J=7.2 \mathrm{~Hz}, 1 \mathrm{H}), 6.92(\mathrm{t}, J=7.6 \mathrm{~Hz}, 1 \mathrm{H}), 3.87(\mathrm{~s}, 3 \mathrm{H}), 2.34(\mathrm{~s}, 3 \mathrm{H}) .{ }^{13} \mathrm{C} \mathrm{NMR}(100$ $\left.\mathrm{MHz}, \mathrm{CDCl}_{3}\right) \delta 189.2,169.2,163.8,153.8,143.5,138.3,132.0,128.6,125.1,125.0$, 125.0, 119.9, 109.9, 94.3, 54.3, 24.1. HRMS (ESI) m/z: $[\mathrm{M}+\mathrm{Na}]^{+}$calcd for $\mathrm{C}_{18} \mathrm{H}_{15} \mathrm{~N}_{2} \mathrm{O}_{5} \mathrm{BrNa}$ 441.0057; found 441.0063. IR (KBr, v) 3451, 3190, 3066, 2957, 1770, 1734, 1678, 1475, 1464, 1386, 1347, 1299, 1272, 1159, 1069, 952, 768, 747, $665 \mathrm{~cm}^{-1}$.

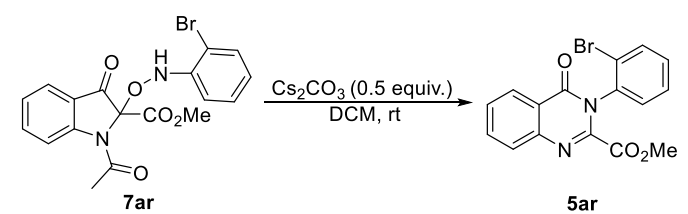

A solution of 7ar $(0.045 \mathrm{mmol})$ and $\mathrm{Cs}_{2} \mathrm{CO}_{3}(0.007 \mathrm{~g}, 0.022 \mathrm{mmol}$.) in DCM (0.5 $\mathrm{mL}$ ) were stirred at room temperature for $3 \mathrm{~d}$. The reaction detected by TLC. The reaction mixture concentrated under reduced pressure. The crude product was purified by column chromatography on silica gel with $\mathrm{V}_{\mathrm{PE}}: \mathrm{V}_{\mathrm{EA}}(3: 1)$ as the eluent to afford the product 5ar in $37 \%$ yield. 


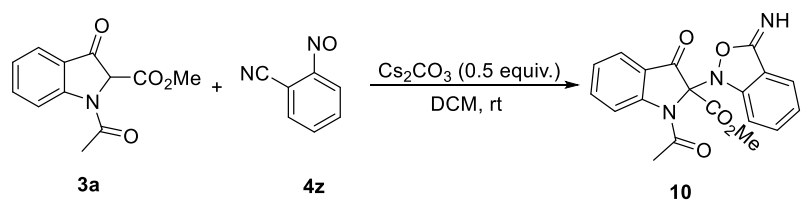

A solution of $\mathbf{3 a}(0.2 \mathrm{mmol})$, 2-nitrosobenzonitrile $\mathbf{4 z}(0.24 \mathrm{mmol})$ and $\mathrm{Cs}_{2} \mathrm{CO}_{3}$ $(0.033 \mathrm{~g}, 0.1 \mathrm{mmol}$.) in DCM $(1 \mathrm{~mL})$ were stirred at room temperature for $15 \mathrm{~min}$. The reaction detected by TLC. The reaction mixture concentrated under reduced pressure. The crude product was purified by column chromatography on silica gel with $V_{P E}: V_{E A}$ (1:1) as the eluent to afford the product $\mathbf{1 0}$ as a light yellow solid. Methyl 1-acetyl-2(3-iminobenzo[c] isoxazol-1(3H)-yl)-3-oxoindoline-2-carboxylate (10). $36 \mathrm{mg}, 50 \%$ yield; m.p. $151-152{ }^{\circ} \mathrm{C} .{ }^{1} \mathrm{H}$ NMR $\left(400 \mathrm{MHz}, \mathrm{CDCl}_{3}\right) \delta$ 8.05-7.65 (m, 4H), 7.62-7.42 (m, 2H), 7.34-7.26 (m, 2H), 3.87 (s, 3H), 2.43 (s, 3H). $\left.{ }^{13} \mathrm{C} \mathrm{NMR} \mathrm{(100} \mathrm{MHz,} \mathrm{CDCl}_{3}\right) \delta$ $187.4,169.2,163.3,163.0,152.9,150.2,138.2,132.7,126.2,125.9,125.1,123.6,122.3$, 119.5, 117.5, 117.1, 54.0, 24.9. HRMS (ESI) m/z: $[\mathrm{M}+\mathrm{Na}]^{+}$calcd for $\mathrm{C}_{19} \mathrm{H}_{15} \mathrm{~N}_{3} \mathrm{O}_{5} \mathrm{Na}$ 388.0904; found 388.0915. IR (KBr, v) 3325, 2926, 2857, 1765, 1734, 1699, 1606, $1468,1326,1305,1259,1085,754 \mathrm{~cm}^{-1}$.

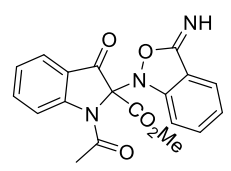

10

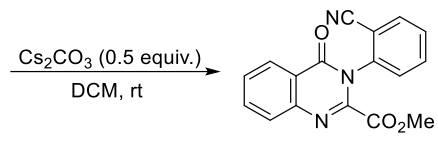

5 az

A solution of $10(0.05 \mathrm{mmol})$ and $\mathrm{Cs}_{2} \mathrm{CO}_{3}(0.008 \mathrm{~g}, 0.025 \mathrm{mmol}$. $)$ in DCM $(0.5 \mathrm{~mL})$ were stirred at room temperature for $24 \mathrm{~h}$. The reaction detected by TLC. The reaction mixture concentrated under reduced pressure. The crude product was purified by column chromatography on silica gel with $\mathrm{V}_{\mathrm{PE}}: \mathrm{V}_{\mathrm{EA}}(3: 1)$ as the eluent to afford the product $\mathbf{5 a z}$ in $78 \%$ yield.

\section{Variation of the EWG at 2-position in 3-indolinone Scaffold. ${ }^{[8]}$}

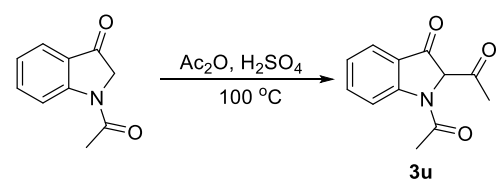

To a solution of 1-acetylindolin-3-one $(2.00 \mathrm{~g}, 11 \mathrm{mmol})$ in acetic anhydride $(20 \mathrm{~mL})$ was added catalytic amount of $\mathrm{H}_{2} \mathrm{SO}_{4}$. Reaction mixture was stirred at $100{ }^{\circ} \mathrm{C}$ for $1 \mathrm{~h}$, evaporated $\mathrm{Ac}_{2} \mathrm{O}$ under reduced pressure. Crude product was dissolved in DCM (100 $\mathrm{mL})$ and washed twice with saturated aqueous $\mathrm{NaHCO}_{3}(2 \times 50 \mathrm{~mL})$, dried over $\mathrm{Na}_{2} \mathrm{SO}_{4}$, filtered and concentrated. The residue was purified by flash column chromatography on silica gel using $\mathrm{V}_{\mathrm{PE}}: \mathrm{V}_{\mathrm{EA}}(3: 1)$ as the eluent, affording the crude product $3 \mathbf{u}$. the 
crude product $3 \mathbf{u}$ recrystallized with DCM/PE affording the pure product $3 \mathbf{u}(0.12 \mathrm{~g}$, $5 \%$ yield) a pale yellow solid, m.p. 165-166 ${ }^{\circ} \mathrm{C}$. 1,1'-(3-oxoindoline-1,2diyl)bis(ethan-1-one) (3u). ${ }^{1} \mathrm{H}$ NMR $\left(400 \mathrm{MHz}, \mathrm{CDCl}_{3}\right) \delta 8.63(\mathrm{~d}, J=6.4 \mathrm{~Hz}, 1 \mathrm{H})$, 7.81-7.59 (m, 2H), $7.22(\mathrm{t}, J=7.6 \mathrm{~Hz}, 1 \mathrm{H}), 5.21(\mathrm{~s}, 1 \mathrm{H}), 2.49(\mathrm{~s}, 3 \mathrm{H}), 2.11(\mathrm{~s}, 3 \mathrm{H}) .{ }^{13} \mathrm{C}$ NMR $\left(100 \mathrm{MHz}, \mathrm{CDCl}_{3}\right) \delta 196.4,189.0,168.3,154.1,138.2,124.7,124.5,118.8,114.8$ 76.6, 27.0, 23.9. HRMS (ESI) m/z: [M + Na] $]^{+}$calcd for $\mathrm{C}_{12} \mathrm{H}_{11} \mathrm{NO}_{3} \mathrm{Na} 240.0631$; found 240.0631. IR (KBr,v) 3427, 2952, 1712, 1677, 1462, 1392, 1351, 1284, $769 \mathrm{~cm}^{-1}$.

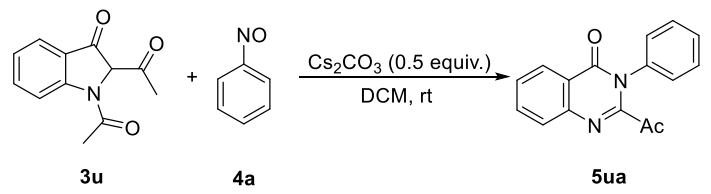

A solution of $3 \mathbf{u}(0.4 \mathrm{mmol})$, nitrosobenzene $4 \mathbf{a}(0.48 \mathrm{mmol})$ and $\mathrm{Cs}_{2} \mathrm{CO}_{3}(0.062 \mathrm{~g}$, $0.2 \mathrm{mmol}$.) in DCM (2 mL) were stirred at room temperature for $22 \mathrm{~h}$. The reaction detected by TLC. The reaction mixture concentrated under reduced pressure. The crude product was purified by column chromatography on silica gel with $\mathrm{V}_{\mathrm{PE}}: \mathrm{V}_{\mathrm{EA}}(5: 1)$ as the eluent to afford the product 5ua as a brown solid. 46 mg, 43\% yield; m.p. 146-147 ${ }^{\circ} \mathrm{C}$. 2-acetyl-3-phenylquinazolin-4(3H)-one (5ua). ${ }^{1} \mathrm{H} \mathrm{NMR}\left(400 \mathrm{MHz}, \mathrm{CDCl}_{3}\right) \delta 8.36$ $(\mathrm{d}, J=8.0 \mathrm{~Hz}, 1 \mathrm{H}), 7.89-7.80(\mathrm{~m}, 2 \mathrm{H}), 7.64-7.58(\mathrm{~m}, 1 \mathrm{H}), 7.54-7.45(\mathrm{~m}, 3 \mathrm{H}), 7.31-7.24$ (m, 2H), 2.54 (s, 3H). ${ }^{13} \mathrm{C}$ NMR (100 MHz, $\left.\mathrm{CDCl}_{3}\right) \delta 194.4,161.6,150.6,146.4,136.6$, 135.0, 129.5, 129.3, 129.0, 128.4, 128.0, 127.5, 122.4, 28.5. HRMS (ESI) m/z: [M + $\mathrm{Na}]^{+}$calcd for $\mathrm{C}_{16} \mathrm{H}_{12} \mathrm{~N}_{2} \mathrm{O}_{2} \mathrm{Na} 287.0791$; found 287.0796. IR (KBr, v) 3416, 2924, 2853, $1716,1691,1581,1562,1362,1340,1169,776 \mathrm{~cm}^{-1}$.

\section{The Other Type Nitroso-Compound were Examined. ${ }^{[9]}$}

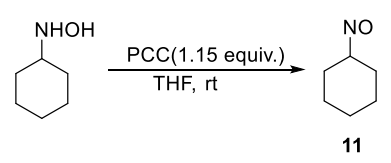

$\mathrm{N}$-cyclohexylhydroxylamine $(0.030 \mathrm{~g}, 0.26 \mathrm{mmol})$ was dissolved in THF $(2 \mathrm{~mL})$ at room temperature and PCC $(0.064 \mathrm{~g}, 0.30 \mathrm{mmol})$ was added. The reaction mixture was stirred at room temperature for $10 \mathrm{~min}$ and detected by TLC. The reaction mixture was filtered through a dry pad of silica gel with $\mathrm{V}_{\mathrm{PE}}$ : $\mathrm{V}_{\mathrm{EA}}(10: 1)$ as the eluent to afford the product Nitrosocyclohexane (11). ${ }^{[9]}$ White solid, $14 \mathrm{mg}$, 46\% yield; m.p. 105-106 ${ }^{\circ} \mathrm{C}$. ${ }^{1} \mathrm{H}$ NMR $\left(400 \mathrm{MHz}, \mathrm{CDCl}_{3}\right) \delta 5.13-5.00(\mathrm{~m}, 1 \mathrm{H}), 1.99-1.80(\mathrm{~m}, 4 \mathrm{H}), 1.75-1.59(\mathrm{~m}, 3 \mathrm{H})$, 1.44-1.30 (m, 2H) 1.28-1.20 (m, 1H). $\left.{ }^{13} \mathrm{C} \mathrm{NMR} \mathrm{(100} \mathrm{MHz,} \mathrm{CDCl}_{3}\right) \delta$ 65.8, 28.4, 25.2, 24.7. HRMS (ESI) m/z: $[\mathrm{M}+\mathrm{Na}]^{+}$calcd for $\mathrm{C}_{6} \mathrm{H}_{11} \mathrm{NONa} 136.0733$; found 136.0736. IR $(\mathrm{KBr}, v)$ 3412, 2925, 2853, 1772, 1690, 1607, 1327, 1304, $759 \mathrm{~cm}^{-1}$. 


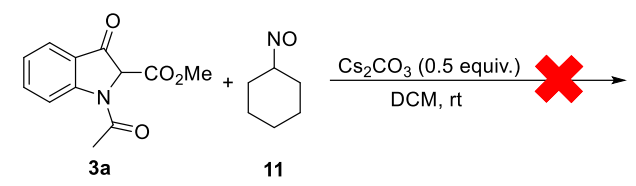

A solution of 3a $(0.0233 \mathrm{~g}, 0.1 \mathrm{mmol}), \mathbf{1 1}(0.0140 \mathrm{~g}, 0.12 \mathrm{mmol})$ and $\mathrm{Cs}_{2} \mathrm{CO}_{3}(0.018$ $\mathrm{g}, 0.05 \mathrm{mmol}$.) in DCM $(0.5 \mathrm{~mL})$ were stirred at room temperature for $2 \mathrm{~d}$. The ring expansion reaction of nitrosocyclohexane and 3a didn't occur.

\section{References}

[1] a) Yarlagadda, S.; Ramesh, B.; Ravikumar Reddy, C.; Srinivas, L.; Sridhar, B.; Subba Reddy, B. V. Org. Lett. 2017, 19, 170. b) Luo, Z.; Sun, G.; Zhou, Z.; Liu, G.; Luan, B.; Lin, Y.; Zhang, L.; Wang, Z. Chem. Commun.; 2018, 54, 13503. c) Zvilichovsky, G.; Gurvich, V. Tetrahedron 1995, 51, 5479.

[2] a) Molander, G. A.; Cavalcanti, L. N. J. Org. Chem. 2012, 77, 4402. b) Purkait, A.; Roy, S. K.; Srivastava, H. K.; Jana, C. K. Org. Lett. 2017, 19, 2540. c) Yamamoto, Y.; Yamamoto, H. J. Am. Chem. Soc. 2004, 126, 4128. d) Jeffrey, J. L.; McClintock, S. P.; Haley, M. M. J. Org. Chem. 2008, 73, 3288.

[3] a) Zhou, B.; Hu, J.; Xu, F.; Chen, Z.; Bai, L.; Fernandez-Salas, E.; Lin, M.; Liu, L.; Yang, C.-Y.; Zhao, Y.; McEachern, D.; Przybranowski, S.; Wen, B.; Sun, D.; Wang, S. J. Med. Chem. 2018, 61, 462. b) Paul, B.; Butterfoss, G. L.; Boswell, M. G.; Douglas Renfrew, P.; Yeung, F. G.; Shah, N. H.; Wolf, C.; Bonneau, R.; Kirshenbaum, K. J. Am. Chem. Soc. 2011, 133, 10910.

[4] Tainaka, K.; Fujitsuka, M.; Takada, T.; Kawai, K.; Majima, T. J. Phys. Chem. B 2010, 114, 14657.

[5] a) Dong, S.; Indukuri, K.; Clive, D. L. J.; Gao, J.-M. Chem. Commun. 2016, 52, 8271. b) Yu, J.; Xu, L.; Hong, D.; Zhang, X.; Liu, J.; Li, D.; Li, J.; Zhou, Y.; Liu, T. Eur. J. Med. Chem. 2019, 161543.

[6] Vacher, B.; Funes, P.; Chopin, P.; Cussac, D.; Heusler, P.; Tourette, A.; Marien, M. J. Med. Chem. 2010, 53, 6986.

[7] Hamaguchi, W.; Masuda, N.; Samizu, K.; Mihara, T.; Takama, K.; Watanabe, T. Chem. Pharm. Bull. 2014, 621200.

[8] a) Velezheva, V. S.; Smushkevich, V. Yu.; Romanova, O. B.; Kurkovskaya, L. N.; Suvorov, N. N. Zh. Org. Khim. 1986, 22, 2434. b) Yarlagadda, S.; Ramesh, B.; Ravikumar Reddy, C.; Srinivas, L.; Sridhar, B.; Subba Reddy, B. V. Org. Lett. 2017, $19,170$. 
[9] Wood, W. W.; Wilkin, J. A. Synth. Commun. 1992, 22, 1683. 


\section{X- ray crystallographic analysis of 5aa, 5sa, 6ta, 7ar and 10 .}

11.1 X-Ray crystallographic analysis of 5aa (CCDC 2075789).

Using the minimum amount of DCM to keep the sample 5aa (30 mg) completely dissolved in the vial. Then, EA (half amount of DCM) and PE (equivalent amount of DCM) were added to the tube. Mixed the solution, and then PE (equivalent amount of DCM) was carefully added on the top of the solution. The resulting solution was sealed with parafilm which has many holes. Allowing the solution slowly evaporate at room temperature, affording the crystal of 5aa. The X-ray crystallographic analysis of 5aa was obtained on a Bruker D8 Venture diffractometer.
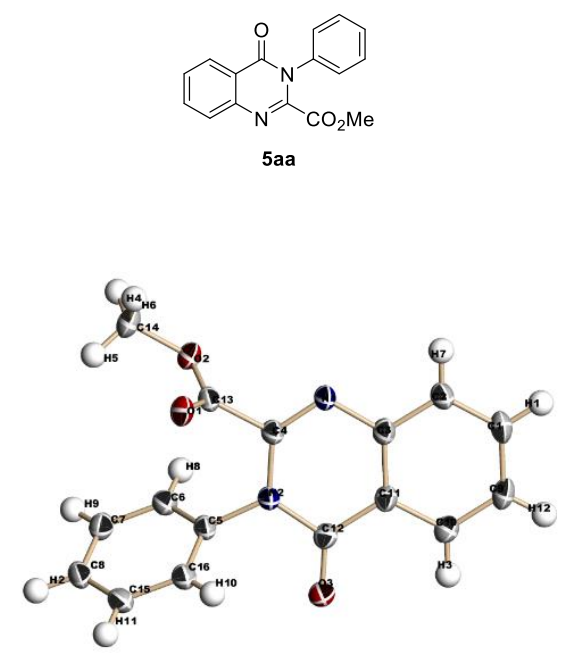

Figure S1. ORTEP drawing of 5aa with $50 \%$ ellipsoid probability. 
Table S1. Crystal data and structure refinement for 5aa.

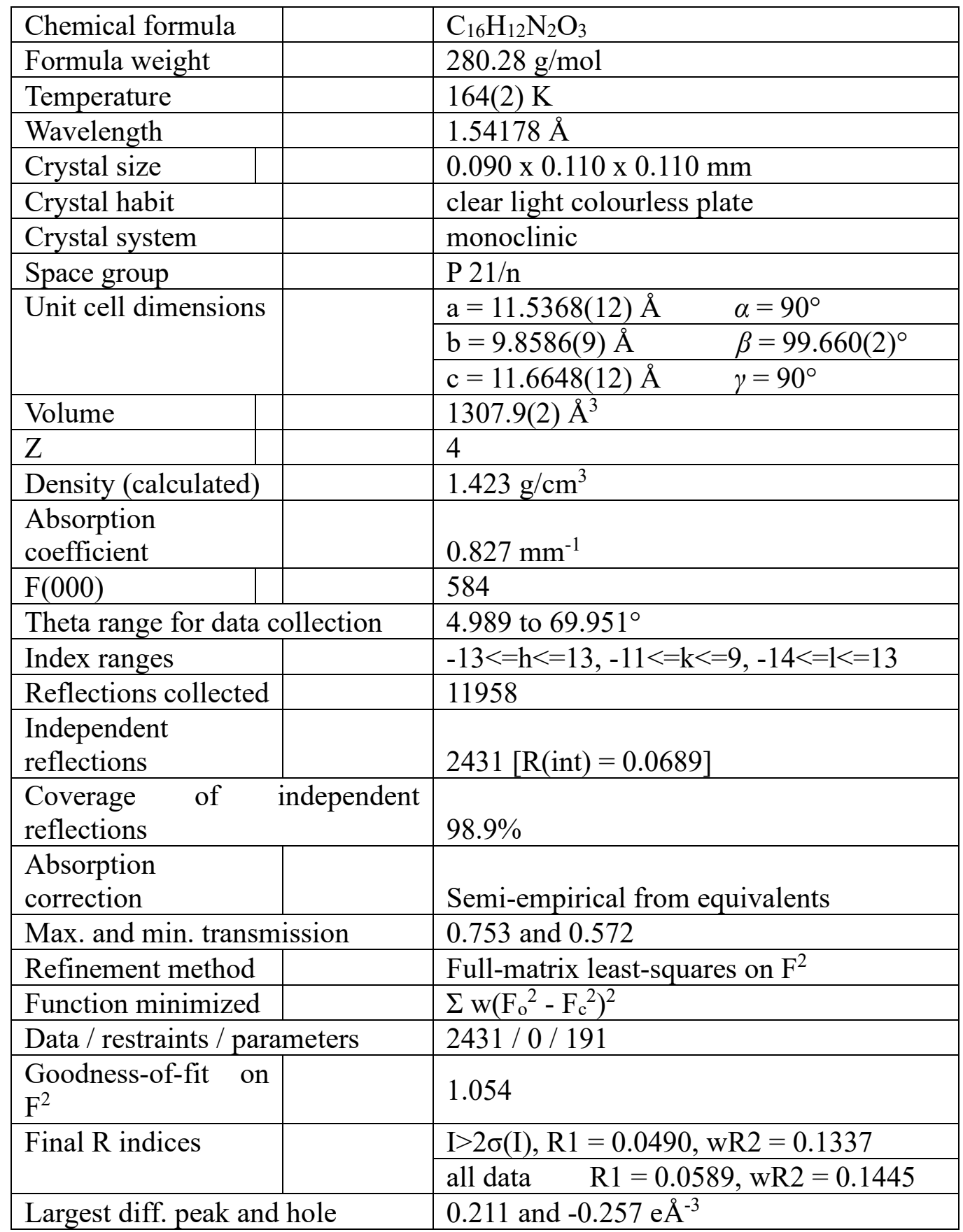


11.2 X-Ray crystallographic analysis of 5sa (CCDC 2075790).

Using the minimum amount of DCM to keep the sample $\mathbf{5 s a}(30 \mathrm{mg})$ completely dissolved in the vial. Then, EA (half amount of DCM) and PE (equivalent amount of DCM) were added to the tube. Mixed the solution, and then PE (equivalent amount of DCM) was carefully added on the top of the solution. The resulting solution was sealed with parafilm which has many holes. Allowing the solution slowly evaporate at room temperature, affording the crystal of 5sa. The X-ray crystallographic analysis of 5sa was obtained on a Bruker D8 Venture diffractometer.

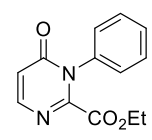

5sa

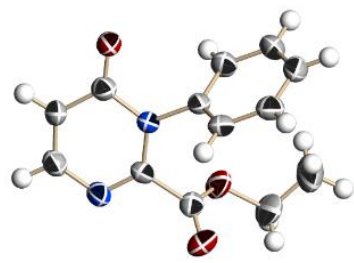

Figure S2. ORTEP drawing of 5sa with 50\% ellipsoid probability. 
Table S2. Crystal data and structure refinement for $\mathbf{5 s a}$.

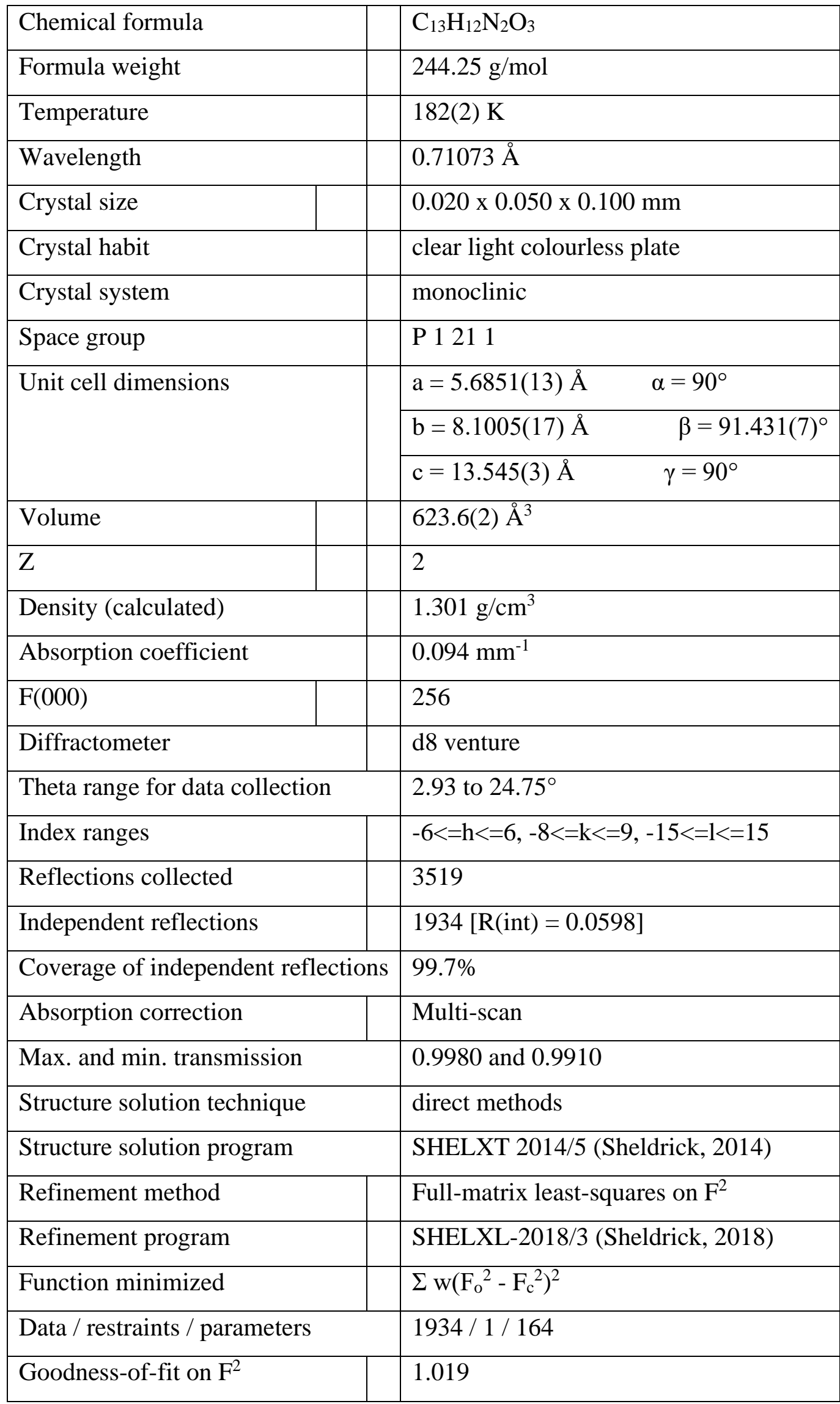




\begin{tabular}{|c|c|}
\hline \multirow[t]{2}{*}{ Final $\mathrm{R}$ indices } & $\mathrm{I}>2 \sigma(\mathrm{I}), \mathrm{R} 1=0.0569, \mathrm{wR} 2=0.1229$ \\
\hline & all data $\quad \mathrm{R} 1=0.0994, \mathrm{wR} 2=0.1493$ \\
\hline \multirow[t]{2}{*}{ Weighting scheme } & $\mathrm{W}=1 /\left[\sigma^{2}\left(\mathrm{Fo}^{2}\right)+(0.0652 \mathrm{P})^{2}\right]$ \\
\hline & where $\mathrm{P}=\left(\mathrm{F}_{\mathrm{o}}^{2}+2 \mathrm{~F}_{\mathrm{c}}^{2}\right) / 3$ \\
\hline Largest diff. peak and hole & 0.160 and $-0.254 \mathrm{e}^{-3}$ \\
\hline R.M.S. deviation from mean & $0.049 \mathrm{e}^{-3}$ \\
\hline
\end{tabular}


11.3 X-Ray crystallographic analysis of 6ta (CCDC 2075791).

Using the minimum amount of DCM to keep the sample 6ta (20 mg) completely dissolved in the vial. Then, EA (half amount of DCM) and PE (equivalent amount of DCM) were added to the tube. Mixed the solution, and then PE (equivalent amount of DCM) was carefully added on the top of the solution. The resulting solution was sealed with parafilm which has many holes. Allowing the solution slowly evaporate at room temperature, affording the crystal of 6ta. The X-ray crystallographic analysis of 6ta was obtained on a Bruker D8 Venture diffractometer.

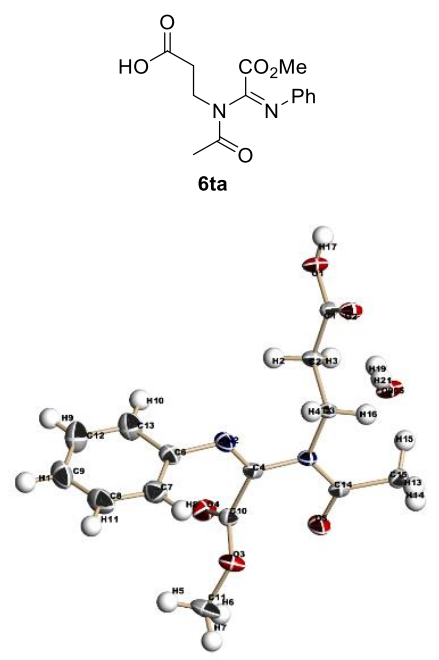

Figure S3. ORTEP drawing of 6 ta with $50 \%$ ellipsoid probability. 
Table S3. Crystal data and structure refinement for 6 ta.

\begin{tabular}{|c|c|}
\hline Chemical formula & $\mathrm{C}_{14} \mathrm{H}_{18} \mathrm{~N}_{2} \mathrm{O}_{6}$ \\
\hline Formula weight & $310.30 \mathrm{~g} / \mathrm{mol}$ \\
\hline Temperature & $238(2) \mathrm{K}$ \\
\hline Wavelength & $1.54178 \AA$ \\
\hline Crystal size & $0.020 \times 0.080 \times 0.210 \mathrm{~mm}$ \\
\hline Crystal habit & clear light colourless plate \\
\hline Crystal system & monoclinic \\
\hline Space group & $\mathrm{P} 121 / \mathrm{n} 1$ \\
\hline \multirow[t]{3}{*}{ Unit cell dimensions } & $\mathrm{a}=13.9652(17) \AA$ \\
\hline & $\begin{array}{l}\mathrm{b}=6.2247(7) \AA \\
109.966(5)^{\circ}\end{array}$ \\
\hline & $\mathrm{c}=18.906(2) \AA \quad \gamma=90^{\circ}$ \\
\hline Volume & $1544.7(3) \AA^{3}$ \\
\hline $\mathrm{Z}$ & 4 \\
\hline Density (calculated) & $1.334 \mathrm{~g} / \mathrm{cm}^{3}$ \\
\hline Absorption coefficient & $0.891 \mathrm{~mm}^{-1}$ \\
\hline $\mathrm{F}(000)$ & 656 \\
\hline Diffractometer & $\mathrm{d} 8$ venture \\
\hline Theta range for data collection & \multirow{2}{*}{$\begin{array}{l}3.44 \text { to } 68.19^{\circ} \\
-16<=\mathrm{h}<=15,-6<=\mathrm{k}<=7,-21<=1<=22\end{array}$} \\
\hline Index ranges & \\
\hline Reflections collected & 11751 \\
\hline Independent reflections & $2797[\mathrm{R}(\mathrm{int})=0.0331]$ \\
\hline Coverage of independen & $98.7 \%$ \\
\hline Absorption correction & Multi-Scan \\
\hline Max. and min. transmiss & 0.9820 and 0.8350 \\
\hline Structure solution techni & direct methods \\
\hline Structure solution progr & SHELXT 2014/5 (Sheldrick, 2014) \\
\hline Refinement method & Full-matrix least-squares on $\mathrm{F}^{2}$ \\
\hline Refinement program & SHELXL-2018/3 (Sheldrick, 2018) \\
\hline Function minimized & $\Sigma \mathrm{w}\left(\mathrm{F}_{\mathrm{o}}^{2}-\mathrm{F}_{\mathrm{c}}^{2}\right)^{2}$ \\
\hline Data / restraints / param & $2797 / 0 / 221$ \\
\hline
\end{tabular}




\begin{tabular}{|l|l|}
\hline Goodness-of-fit on $\mathrm{F}^{2}$ & 1.046 \\
\hline Final R indices & $\begin{array}{l}2586 \text { data; } \mathrm{I}>2 \sigma(\mathrm{I}), \mathrm{R} 1=0.0338, \mathrm{wR} 2 \\
=0.0872\end{array}$ \\
\cline { 2 - 2 } & $\begin{array}{l}\text { all data } \mathrm{R} 1=0.0366, \mathrm{wR} 2= \\
0.0901\end{array}$ \\
\hline Weighting scheme & $\mathrm{w}=1 /\left[\sigma^{2}\left(\mathrm{Fo}^{2}\right)+(0.0410 \mathrm{P})^{2}+0.5748 \mathrm{P}\right]$ \\
\cline { 2 - 2 } & where $\mathrm{P}=\left(\mathrm{F}_{\mathrm{o}}^{2}+2 \mathrm{~F}_{\mathrm{c}}^{2}\right) / 3$ \\
\hline Largest diff. peak and hole & 0.330 and $-0.235 \mathrm{e}^{-3}$ \\
\hline R.M.S. deviation from mean & $0.040 \mathrm{e}^{-3}$ \\
\hline
\end{tabular}


11.4 X-Ray crystallographic analysis of 7ar (CCDC 2075792).

Using the minimum amount of DCM to keep the sample 7ar (20 mg) completely dissolved in the vial. Then, EA (half amount of DCM) and PE (equivalent amount of DCM) were added to the tube. Mixed the solution, and then PE (equivalent amount of DCM) was carefully added on the top of the solution. The resulting solution was sealed with parafilm which has many holes. Allowing the solution slowly evaporate at room temperature, affording the crystal of 7ar. The X-ray crystallographic analysis of 7ar was obtained on a Bruker D8 Venture diffractometer.

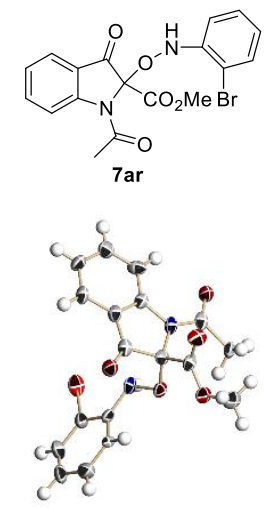

Figure S4. ORTEP drawing of 7ar with $50 \%$ ellipsoid probability. 
Table S4. Crystal data and structure refinement for 7ar.

\begin{tabular}{|c|c|}
\hline Chemical formula & $\mathrm{C}_{18} \mathrm{H}_{15} \mathrm{~N}_{2} \mathrm{O}_{5} \mathrm{Br}$ \\
\hline Formula weight & $419.23 \mathrm{~g} / \mathrm{mol}$ \\
\hline Temperature & $175(2) \mathrm{K}$ \\
\hline Wavelength & $1.54178 \AA$ \\
\hline Crystal size & $0.170 \times 0.140 \times 0.080 \mathrm{~mm}$ \\
\hline Crystal system & triclinic \\
\hline Space group & $\mathrm{P}-1$ \\
\hline Unit cell dimensions & $\mathrm{a}=8.5126(17) \AA \quad \alpha=91.093(9)^{\circ}$ \\
\hline & $\mathrm{b}=9.696(2) \AA \quad \beta=98.458(9)^{\circ}$ \\
\hline & $\mathrm{c}=11.927(2) \AA \quad \gamma=115.152(8)^{\circ}$ \\
\hline Volume & $877.7(3) \AA^{3}$ \\
\hline $\mathrm{Z}$ & 2 \\
\hline Density (calculated) & $1.586 \mathrm{~g} / \mathrm{cm}^{3}$ \\
\hline Absorption coefficient & $3.485 \mathrm{~mm}-1$ \\
\hline $\mathrm{F}(000)$ & 424 \\
\hline Theta range for data collection & 3.762 to $67.721^{\circ}$ \\
\hline Reflections collected & $8882 / 3041[\mathrm{R}($ int $)=0.0687]$ \\
\hline Refinement method & Full-matrix least-squares on $\mathrm{F}^{2}$ \\
\hline Data / restraints / parameters & $3041 / 1 / 241$ \\
\hline Goodness-of-fit on $\mathrm{F}^{2}$ & 1.150 \\
\hline Final $\mathrm{R}$ indices & $\mathrm{I}>2 \sigma(\mathrm{I}), \mathrm{R} 1=0.1356, \mathrm{wR} 2=0.3909$ \\
\hline & all data $\quad \mathrm{R} 1=0.1411, \mathrm{wR} 2=0.3928$ \\
\hline Largest diff. peak and hole & 2.922 and $-0.916 \mathrm{e}^{\circ}{ }^{-3}$ \\
\hline
\end{tabular}


11.5 X-Ray crystallographic analysis of $\mathbf{1 0}$ (CCDC 2084083).

Using the minimum amount of DCM to keep the sample $\mathbf{1 0}(20 \mathrm{mg})$ completely dissolved in the vial. Then, EA (half amount of DCM) and PE (equivalent amount of DCM) were added to the tube. Mixed the solution, and then PE (equivalent amount of DCM) was carefully added on the top of the solution. The resulting solution was sealed with parafilm which has many holes. Allowing the solution slowly evaporate at room temperature, affording the crystal of $\mathbf{1 0}$. The X-ray crystallographic analysis of $\mathbf{1 0}$ was obtained on a Bruker D8 Venture diffractometer.

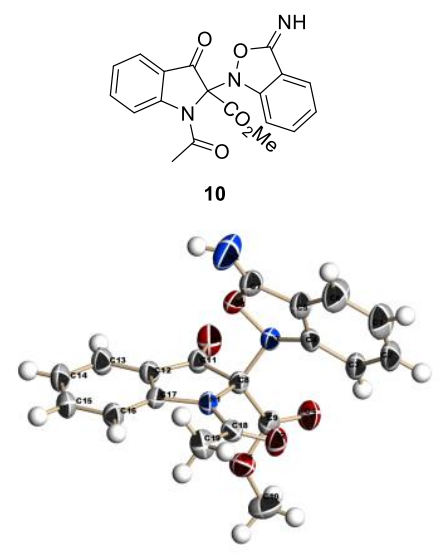

Figure S5. ORTEP drawing of $\mathbf{1 0}$ with $50 \%$ ellipsoid probability. 
Table S5. Crystal data and structure refinement for $\mathbf{1 0 .}$

\begin{tabular}{|c|c|}
\hline Chemical formula & $\mathrm{C}_{19} \mathrm{H}_{15} \mathrm{~N}_{3} \mathrm{O}_{5}$ \\
\hline Formula weight & $365.34 \mathrm{~g} / \mathrm{mol}$ \\
\hline Temperature & $300(2) \mathrm{K}$ \\
\hline Wavelength & $0.71073 \AA$ \\
\hline Crystal size & $0.100 \times 0.250 \times 0.320 \mathrm{~mm}$ \\
\hline Crystal habit & clear light colourless block \\
\hline Crystal system & triclinic \\
\hline Space group & $\mathrm{P}-1$ \\
\hline Unit cell dimensions & $\begin{array}{ll}\mathrm{a}=7.9901(19) \AA & \alpha=78.966(7)^{\circ} \\
\mathrm{b}=8.4240(18) \AA & \beta=82.691(8)^{\circ} \\
\mathrm{c}=14.297(3) \AA & \gamma=63.916(8)^{\circ}\end{array}$ \\
\hline Volume & $847.3(3) \AA^{3}$ \\
\hline $\mathrm{Z}$ & 2 \\
\hline Density (calculated) & $1.432 \mathrm{~g} / \mathrm{cm}^{3}$ \\
\hline Absorption coefficient & $0.106 \mathrm{~mm}^{-1}$ \\
\hline $\mathrm{F}(000)$ & 380 \\
\hline Diffractometer & d8 venture \\
\hline Theta range for data collection & 2.72 to $26.52^{\circ}$ \\
\hline Index ranges & $-10<=\mathrm{h}<=9,-10<=\mathrm{k}<=10,-17<=\mathrm{l}<=17$ \\
\hline Reflections collected & 12868 \\
\hline Independent reflections & $3460[\mathrm{R}$ (int) $=0.0492]$ \\
\hline Coverage of independent reflections & $98.50 \%$ \\
\hline Absorption correction & Multi-Scan \\
\hline Max. and min. transmission & 0.9890 and 0.9670 \\
\hline Structure solution technique & direct methods \\
\hline Structure solution program & SHELXT 2018/2 (Sheldrick, 2018) \\
\hline Refinement method & Full-matrix least-squares on $\mathrm{F}^{2}$ \\
\hline Refinement program & SHELXL-2018/3 (Sheldrick, 2018) \\
\hline Function minimized & $\Sigma \mathrm{w}\left(\mathrm{Fo}^{2}-\mathrm{Fc}^{2}\right)^{2}$ \\
\hline Data / restraints / parameters & $3460 / 0 / 249$ \\
\hline Goodness-of-fit on $\mathrm{F}^{2}$ & 1.012 \\
\hline \multirow[t]{2}{*}{ Final $\mathrm{R}$ indices } & $\begin{array}{l}2980 \text { data; } \mathrm{I}>2 \sigma(\mathrm{I}) \\
\mathrm{R} 1=0.0546, \mathrm{wR} 2=0.1407\end{array}$ \\
\hline & $\begin{array}{l}\text { all data } \\
\mathrm{R} 1=0.0608, \mathrm{wR} 2=0.1493\end{array}$ \\
\hline \multirow[t]{2}{*}{ Weighting scheme } & $\mathrm{W}=1 /\left[\sigma^{2}\left(\mathrm{~F}_{\mathrm{o}}^{2}\right)+(0.0885 \mathrm{P})^{2}+0.1990 \mathrm{P}\right]$ \\
\hline & where $\mathrm{P}=\left(\mathrm{F}_{\mathrm{o}}^{2}+2 \mathrm{~F}_{\mathrm{c}}^{2}\right) / 3$ \\
\hline Largest diff. peak and hole & 0.401 and $-0.328 \mathrm{e}^{-3}$ \\
\hline R.M.S. deviation from mean & $0.086 \mathrm{e}^{-3}$ \\
\hline
\end{tabular}


12. NMR Spectra of the New Reactants.

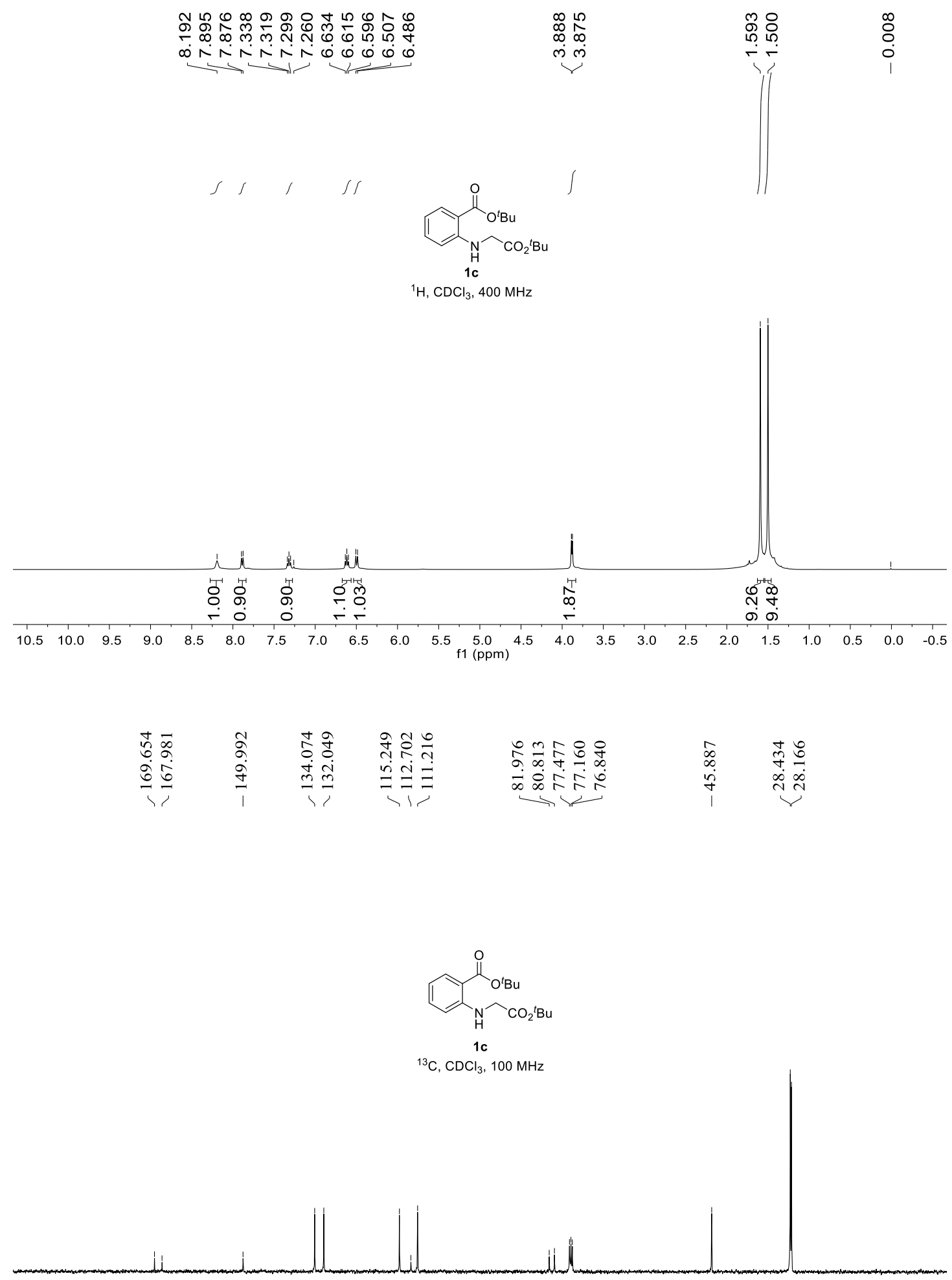

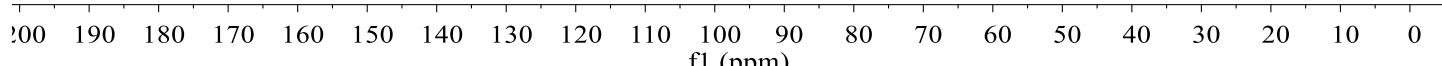



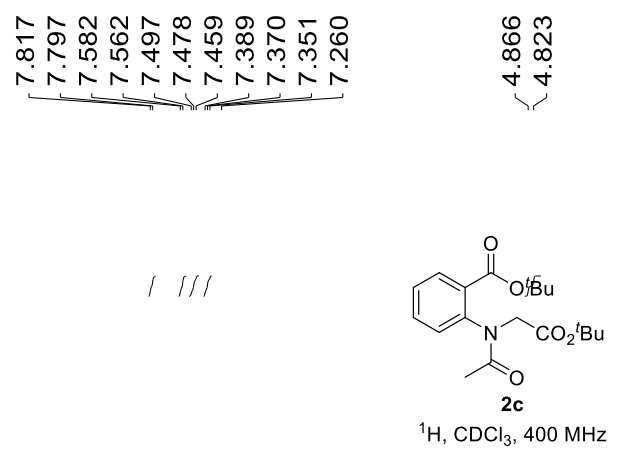

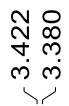

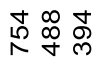

NNNNNNNN

$1 / 11$

L似促

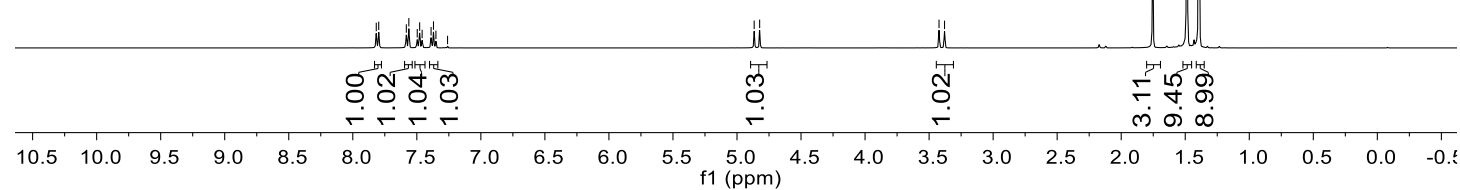

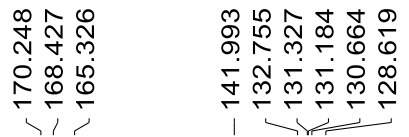

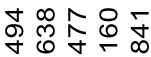

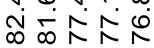

$\underset{\substack{\infty \\ \infty}}{\substack{\infty \\ \hdashline}}$

$\bar{్} \stackrel{\infty}{\mathbb{0}}$

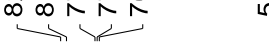

$\stackrel{\infty}{1}$ ล

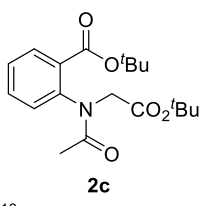

${ }^{13} \mathrm{C}, \mathrm{CDCl}_{3}, 100 \mathrm{MHz}$

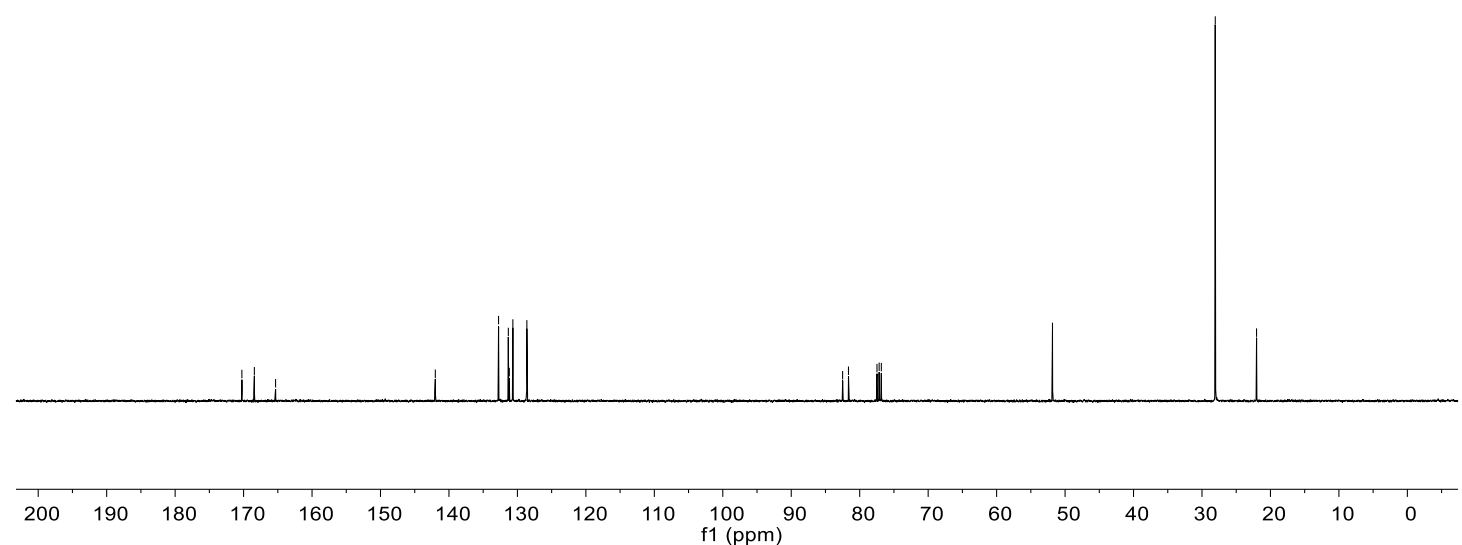




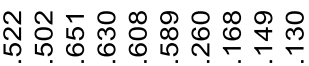

$\infty \infty^{\circ}+\hat{N}+\hat{N}$

m
$\infty$
$j$
$j$

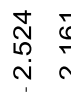

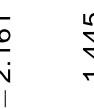
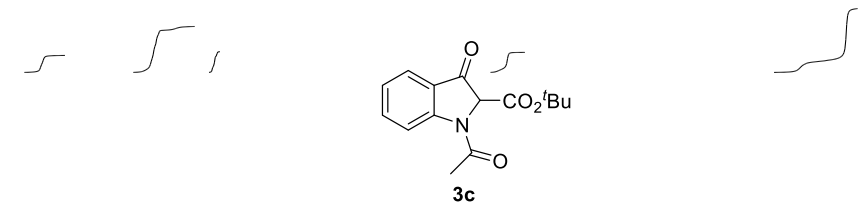

${ }^{1} \mathrm{H}, \mathrm{CDCl}_{3}, 400 \mathrm{MHz}$

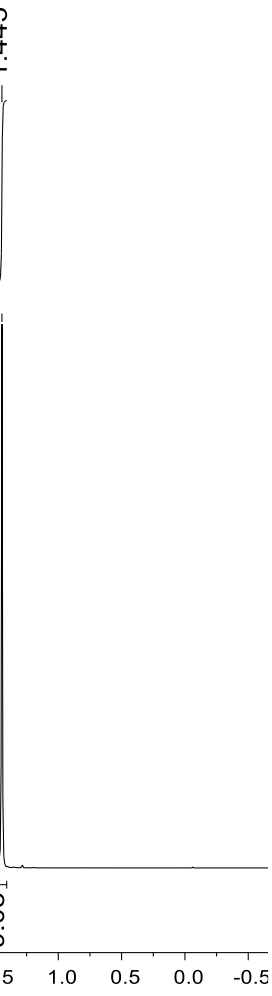

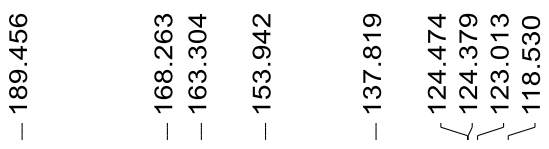

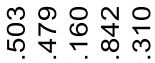

赫定旦

战

N

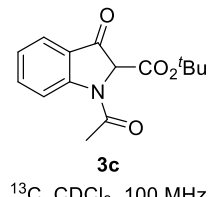

${ }^{13} \mathrm{C}, \mathrm{CDCl}_{3}, 100 \mathrm{MHz}$

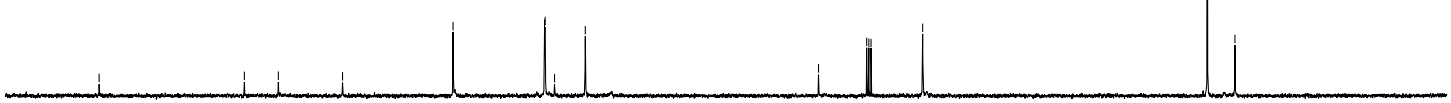

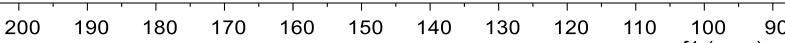

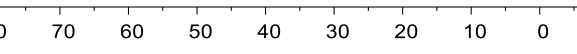




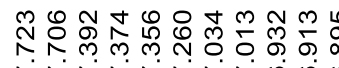

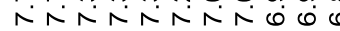

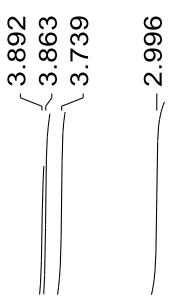

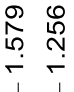

¿̊
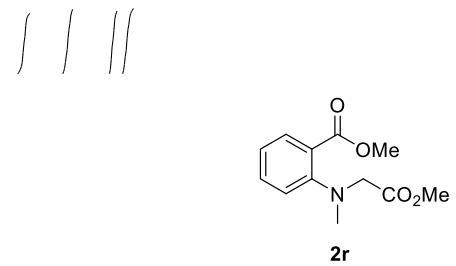

${ }^{1} \mathrm{H}, \mathrm{CDCl}_{3}, 400 \mathrm{MHz}$
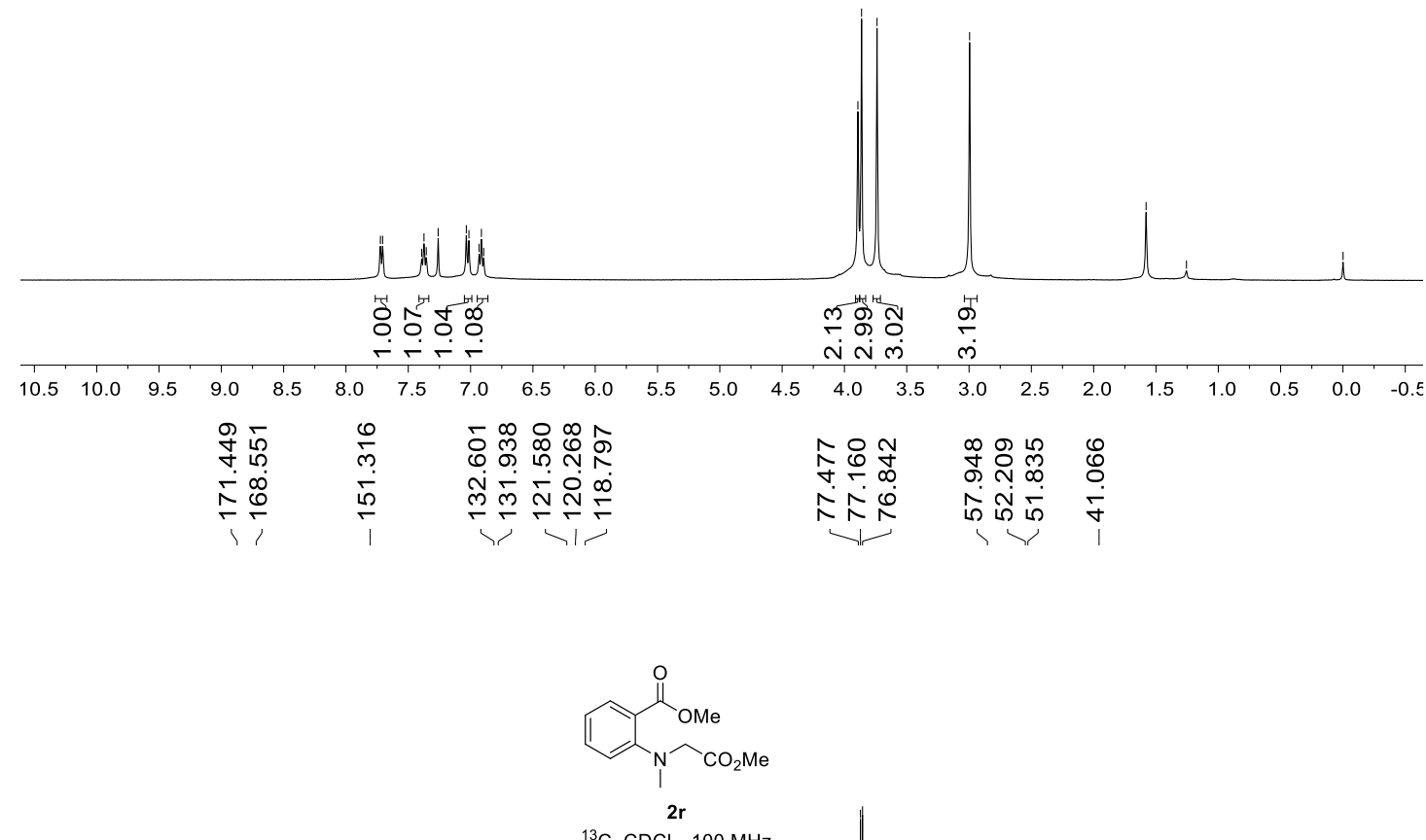

${ }^{13} \mathrm{C}, \mathrm{CDCl}_{3}, 100 \mathrm{MHz}$

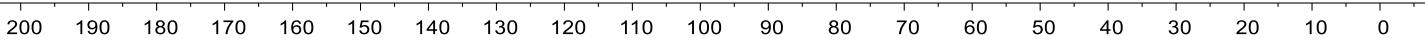




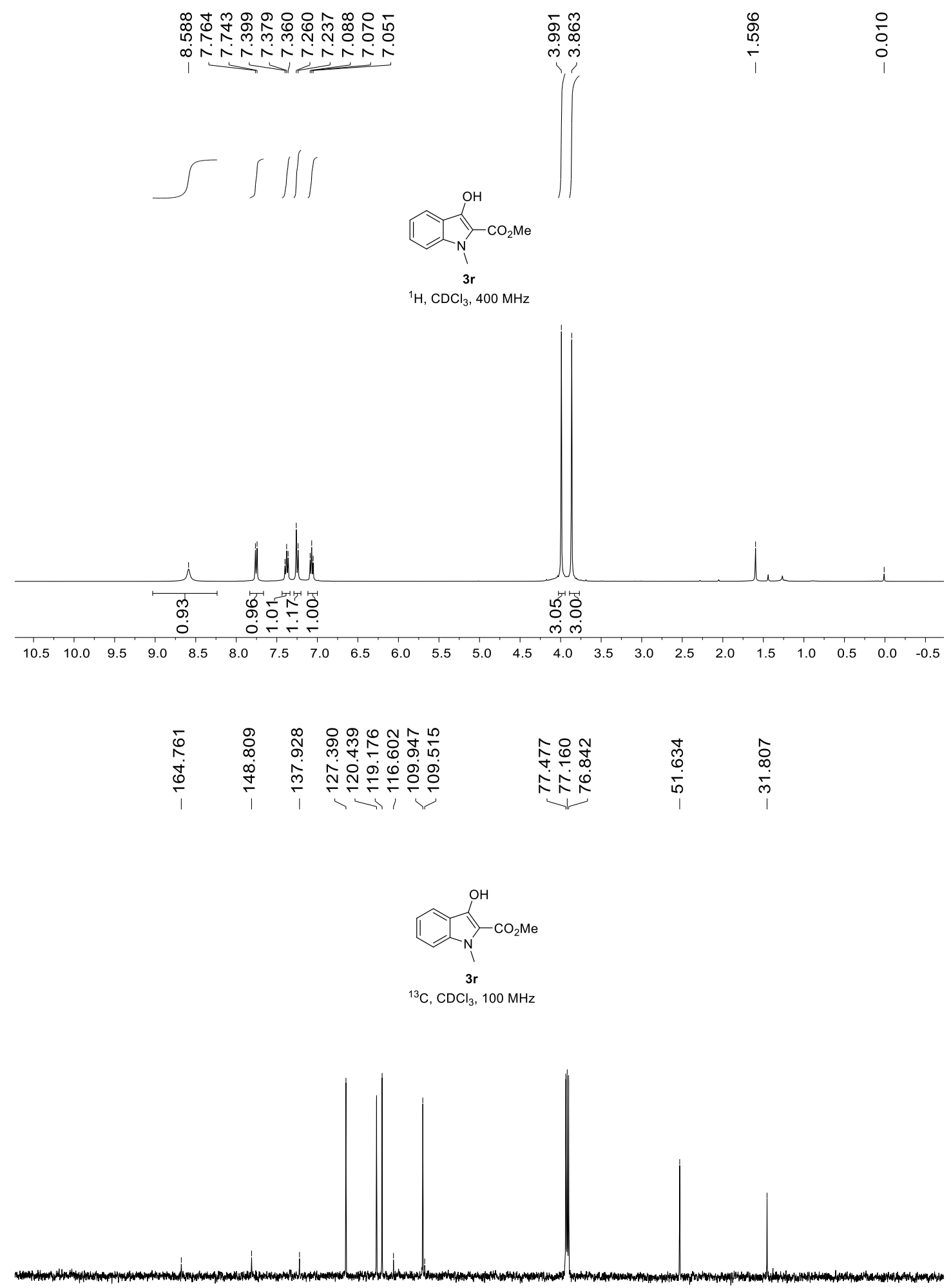

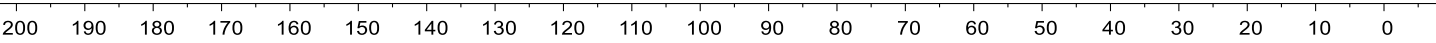


ํㅜㅇ

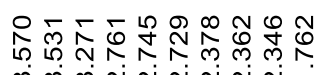

m m m

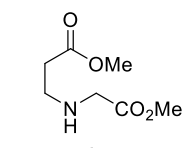

, , मा,

${ }^{1} \mathrm{H}, \mathrm{CDCl}_{3}, 400 \mathrm{MHz}$

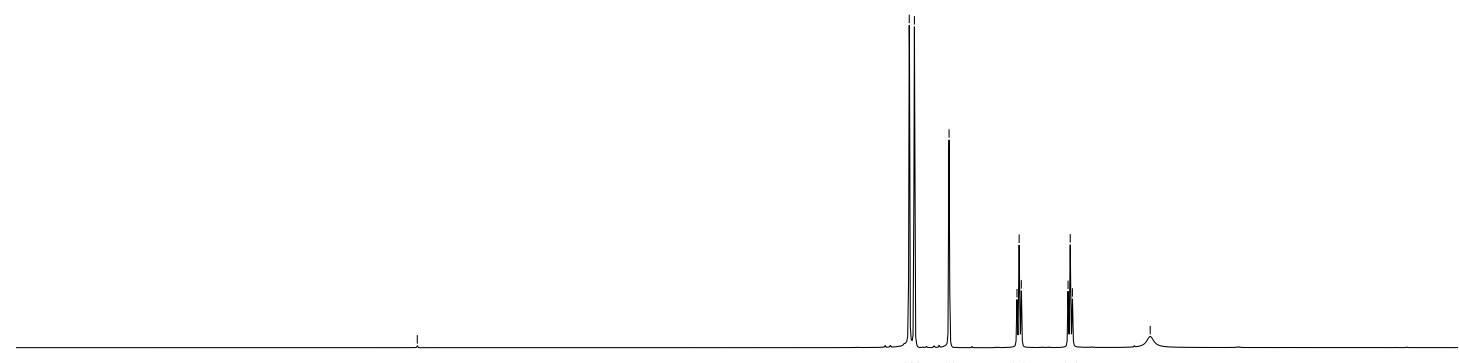

ठำ
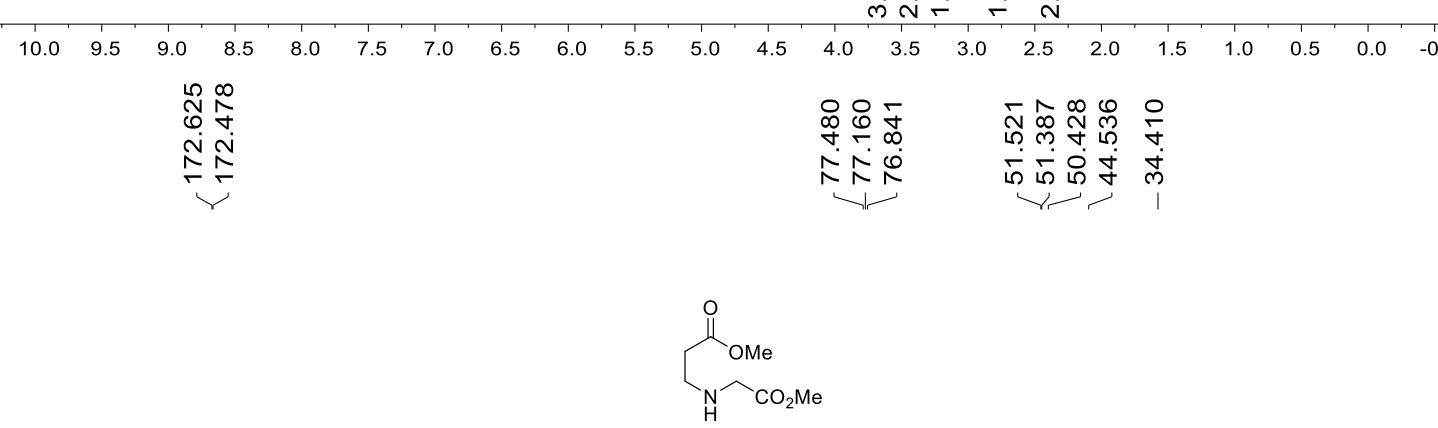

$1 \mathrm{t}$

${ }^{13} \mathrm{C}, \mathrm{CDCl}_{3}, 100 \mathrm{MHz}$

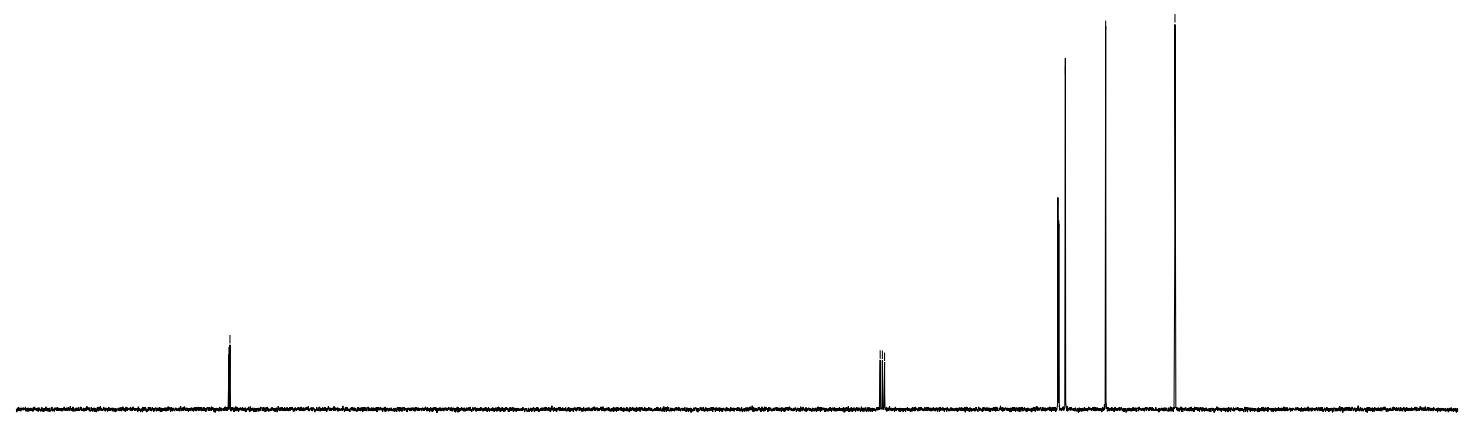

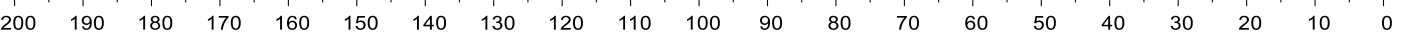




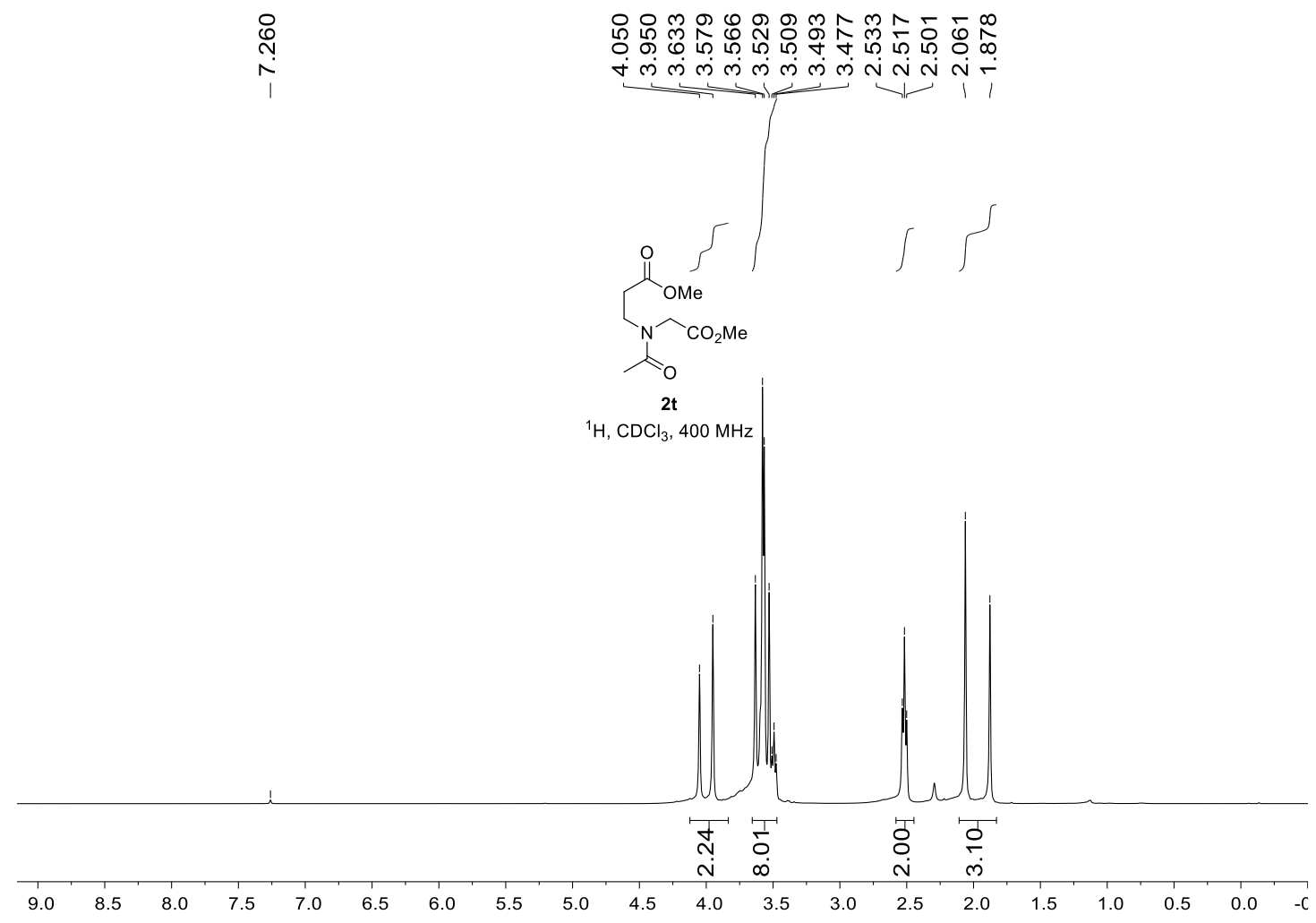

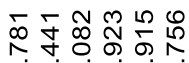

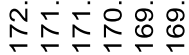

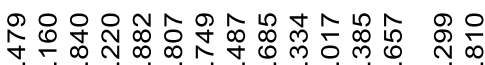

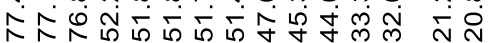

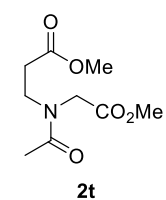

${ }^{13} \mathrm{C}, \mathrm{CDCl}_{3}, 100 \mathrm{MHz}$

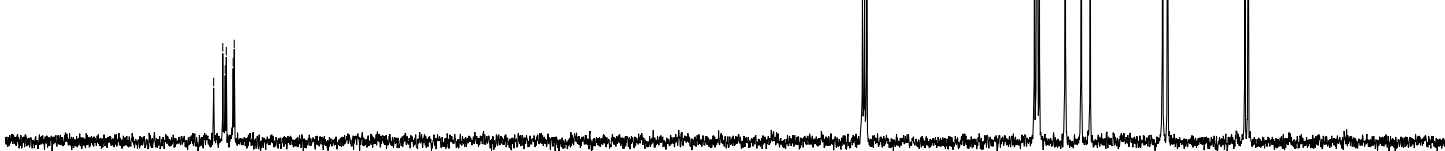

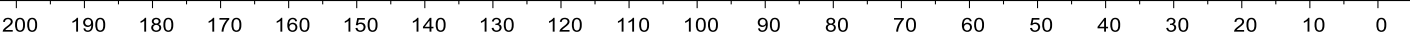



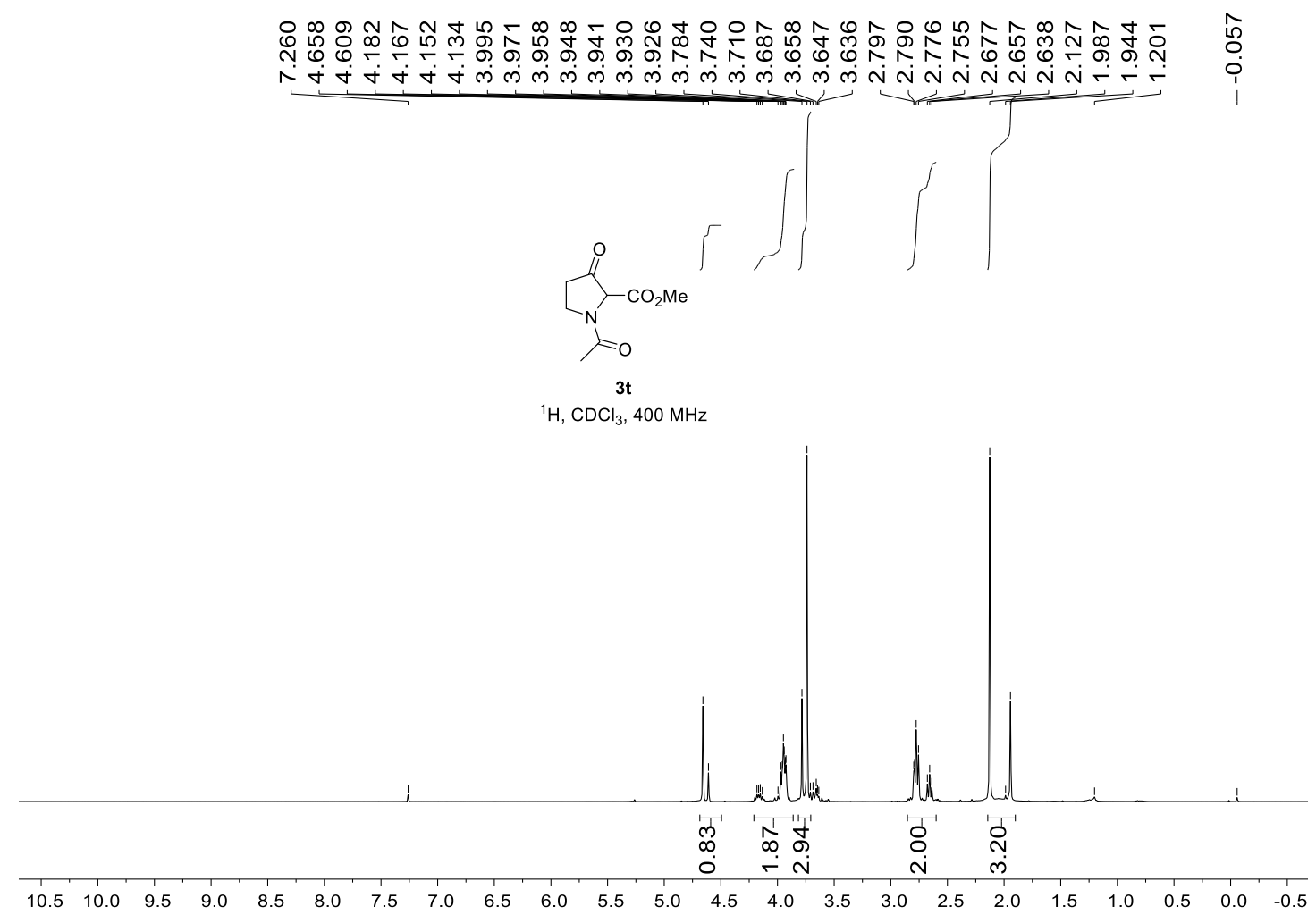

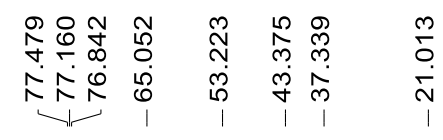

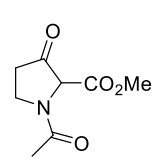

$3 \mathbf{t}$

${ }^{13} \mathrm{C}, \mathrm{CDCl}_{3}, 100 \mathrm{MHz}$

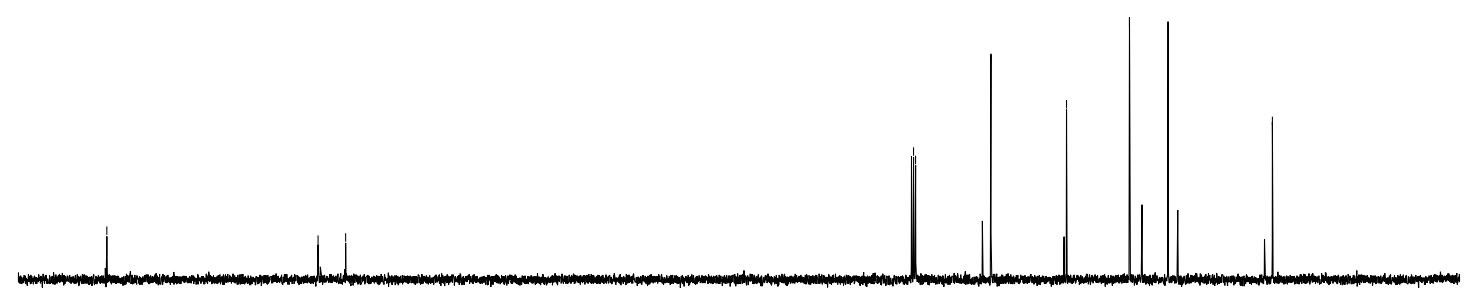

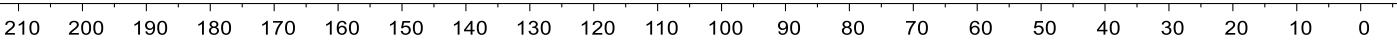


NMR Spectra of the 2,3-disubstituted quinazolinone derivatives.
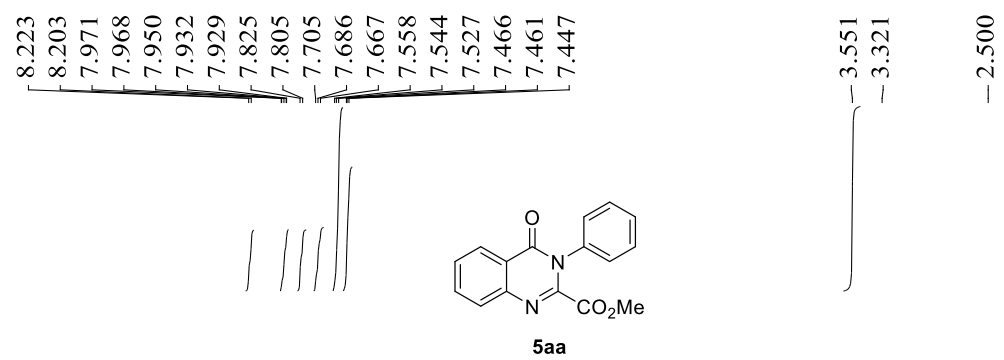

${ }^{1} \mathrm{H}, \mathrm{DMSO}-\mathrm{d} 6,400 \mathrm{MHz}$
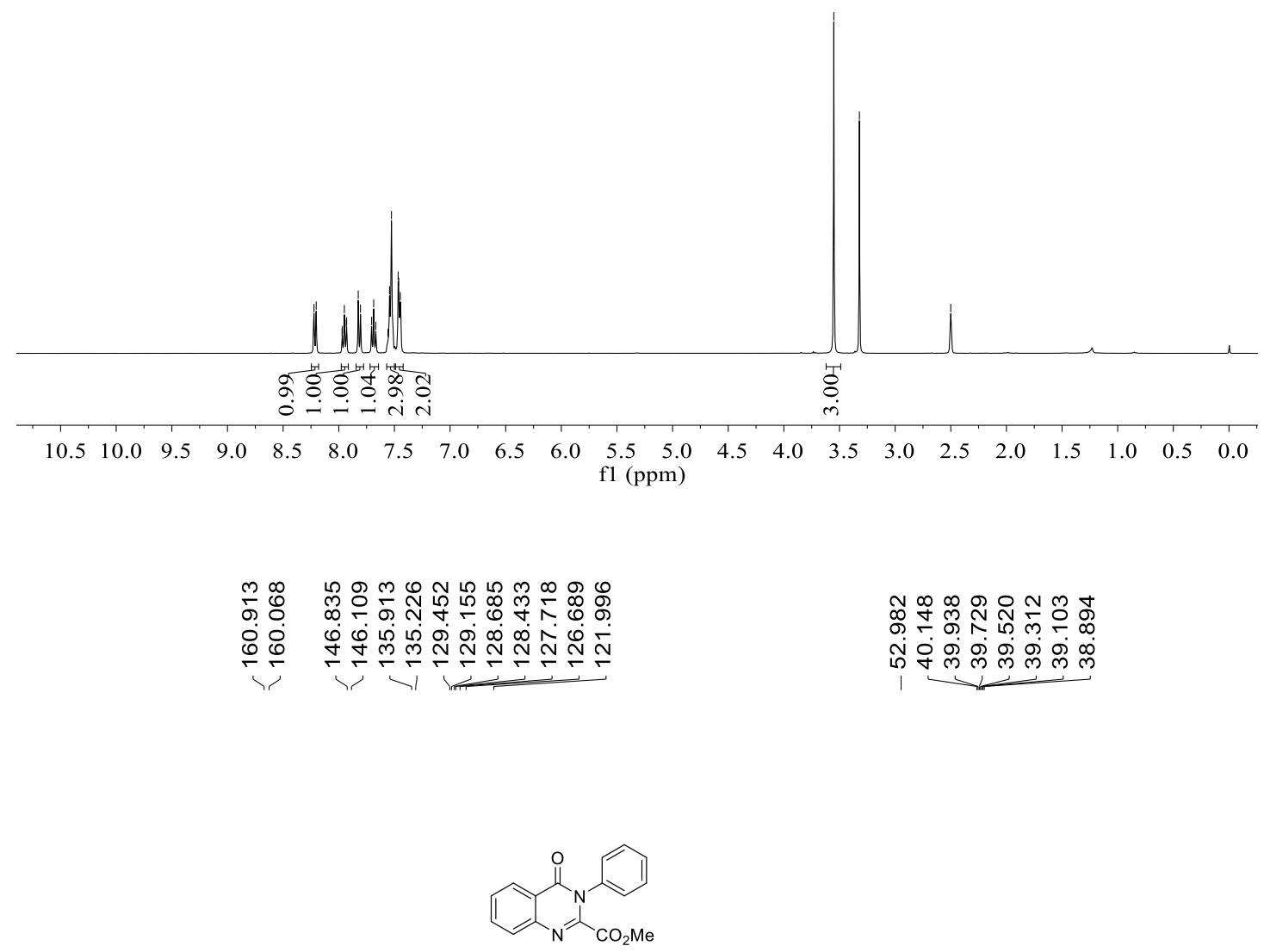

5 aa

${ }^{13} \mathrm{C}, \mathrm{DMSO}-\mathrm{d} 6,100 \mathrm{MHz}$

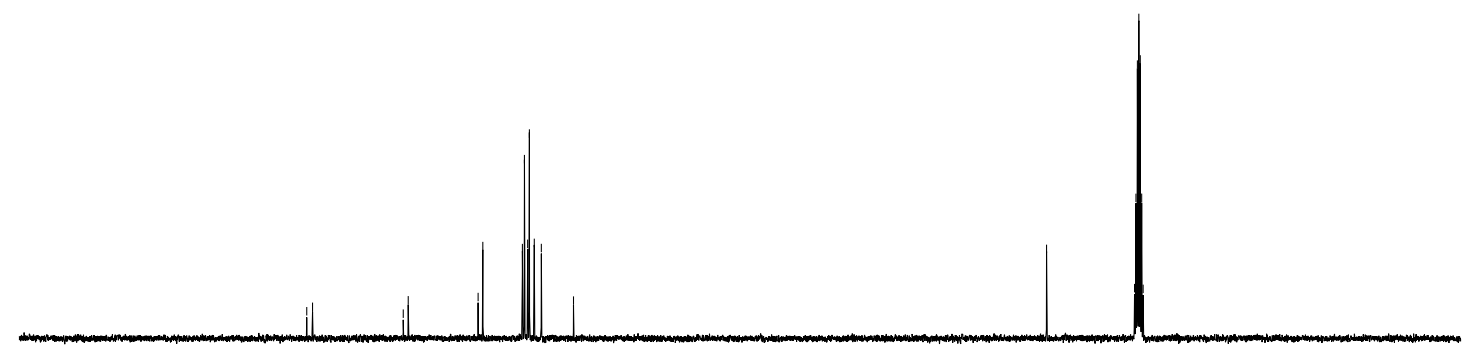

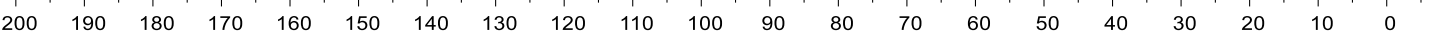




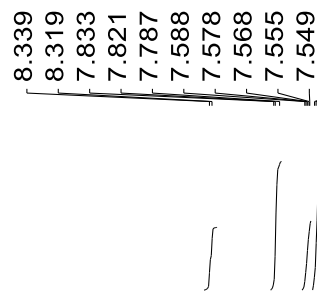

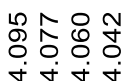

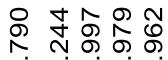

i.

$\stackrel{6}{0}$

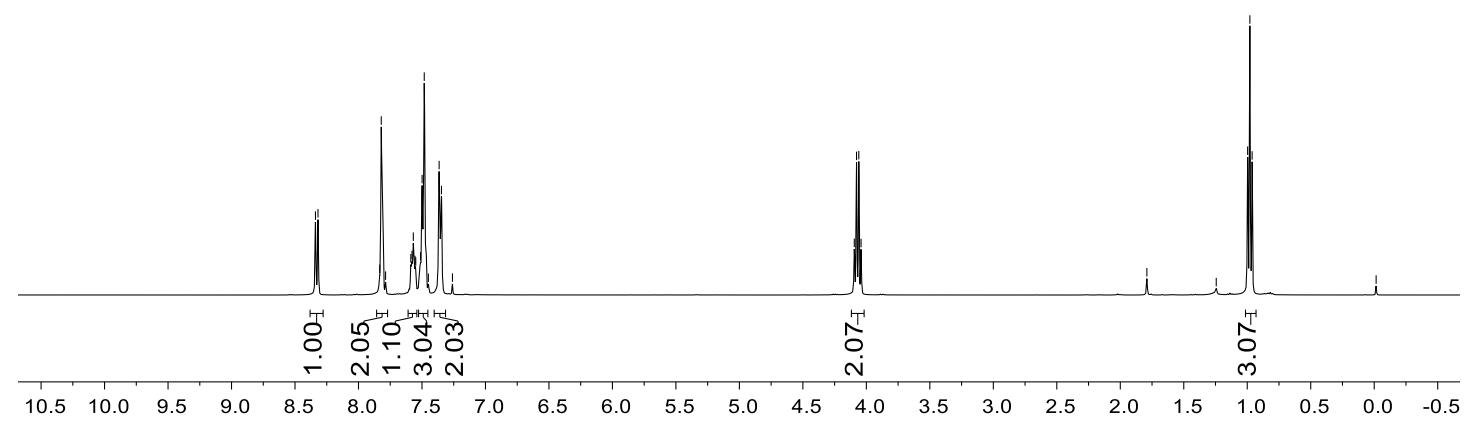

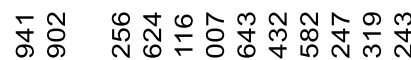

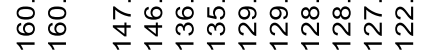

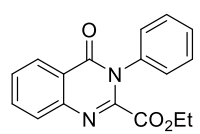

5 ba

${ }^{13} \mathrm{C}, \mathrm{CDCl}_{3}, 100 \mathrm{MHz}$

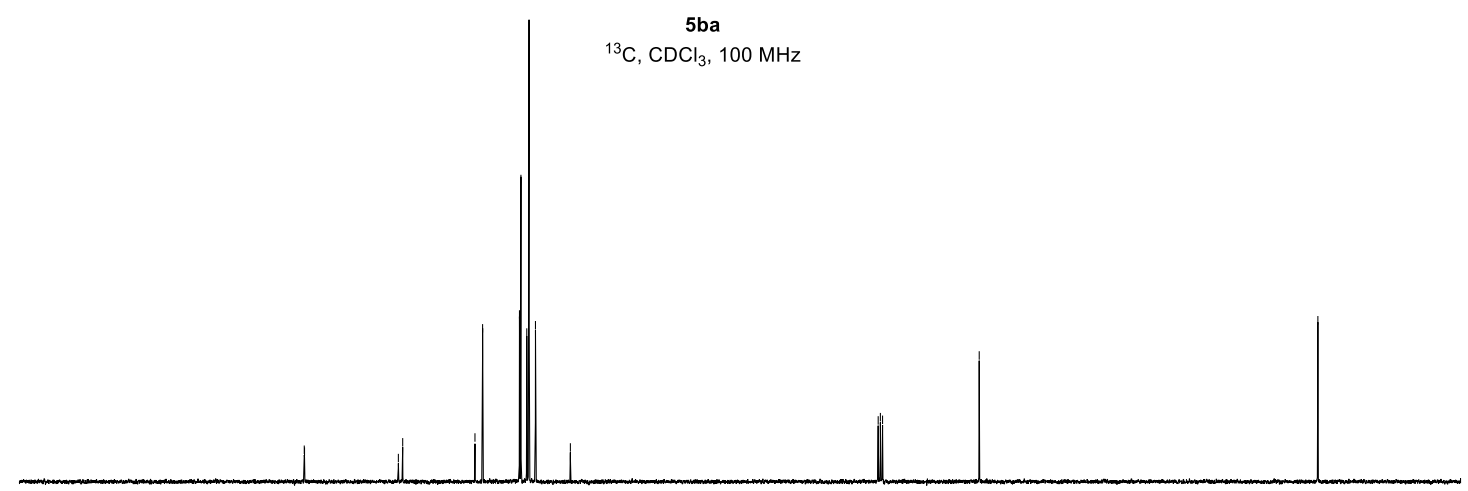

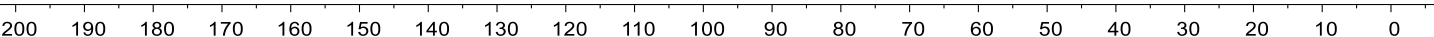




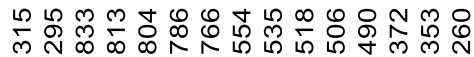

$\infty \infty N+N$ N
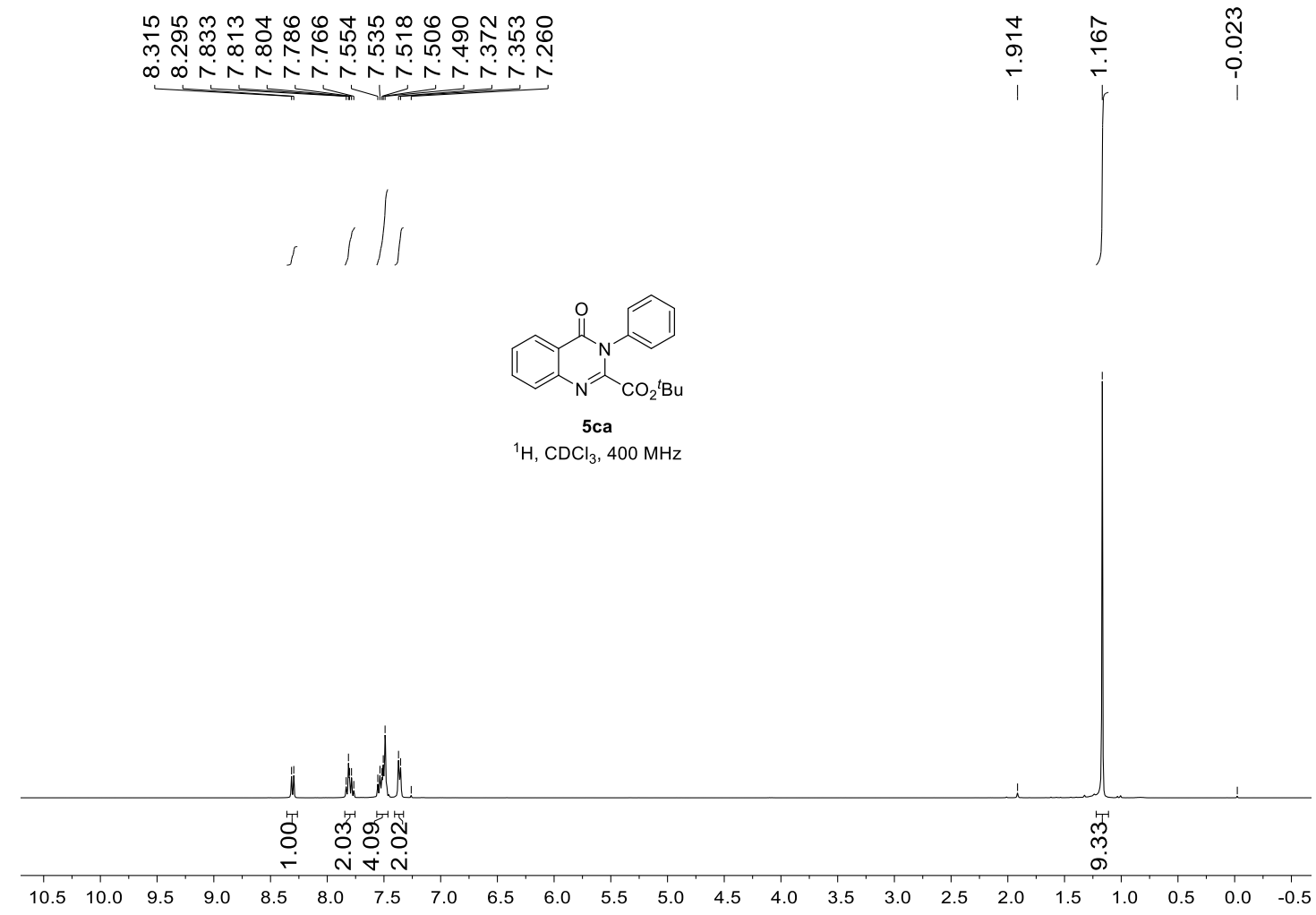

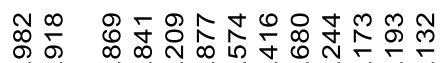

家官

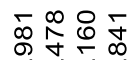

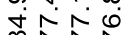

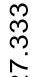

กั่

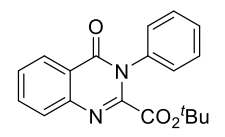

5 ca

${ }^{13} \mathrm{C}, \mathrm{CDCl}_{3}, 100 \mathrm{MHz}$

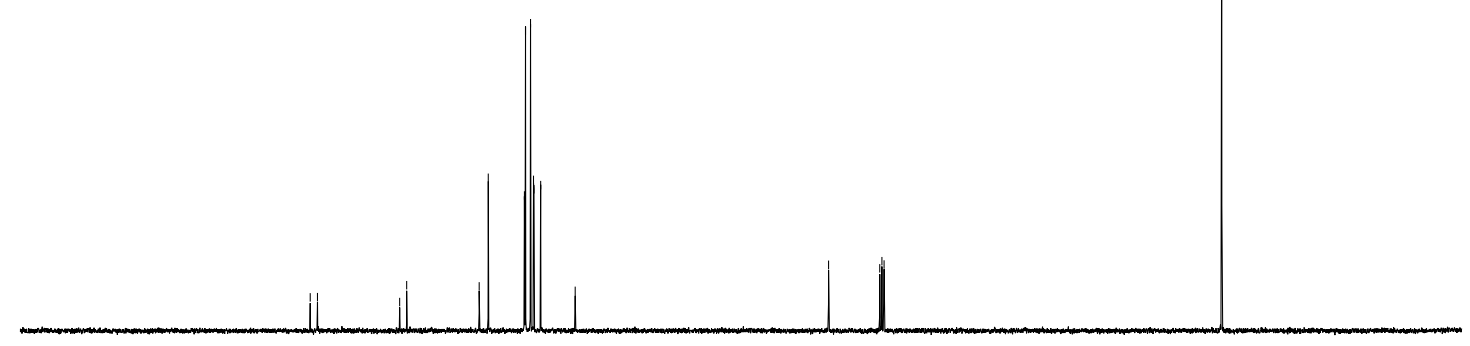

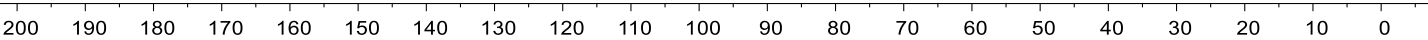



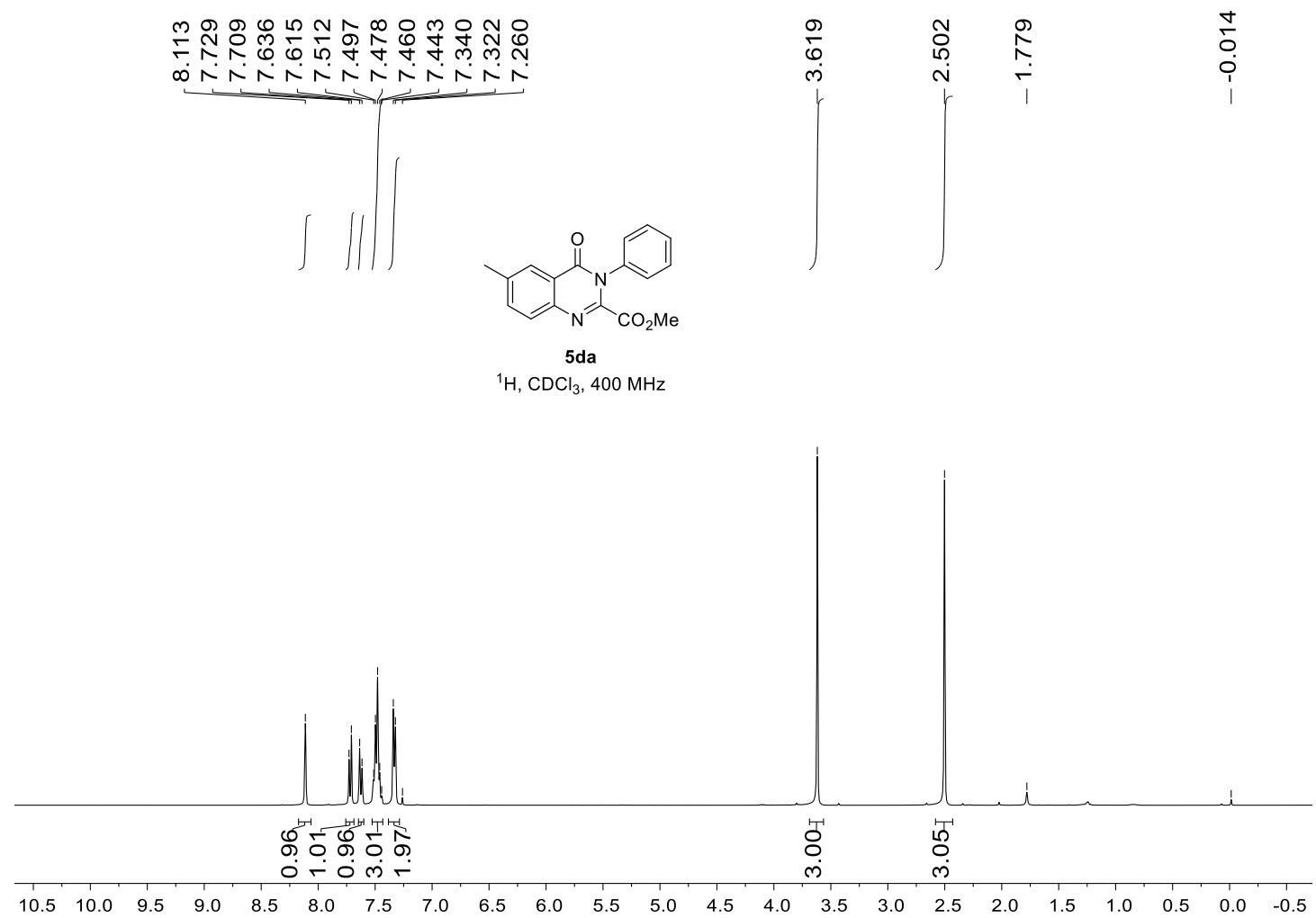

ฟิ่

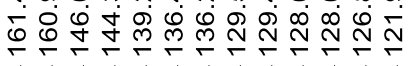

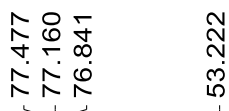

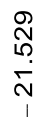

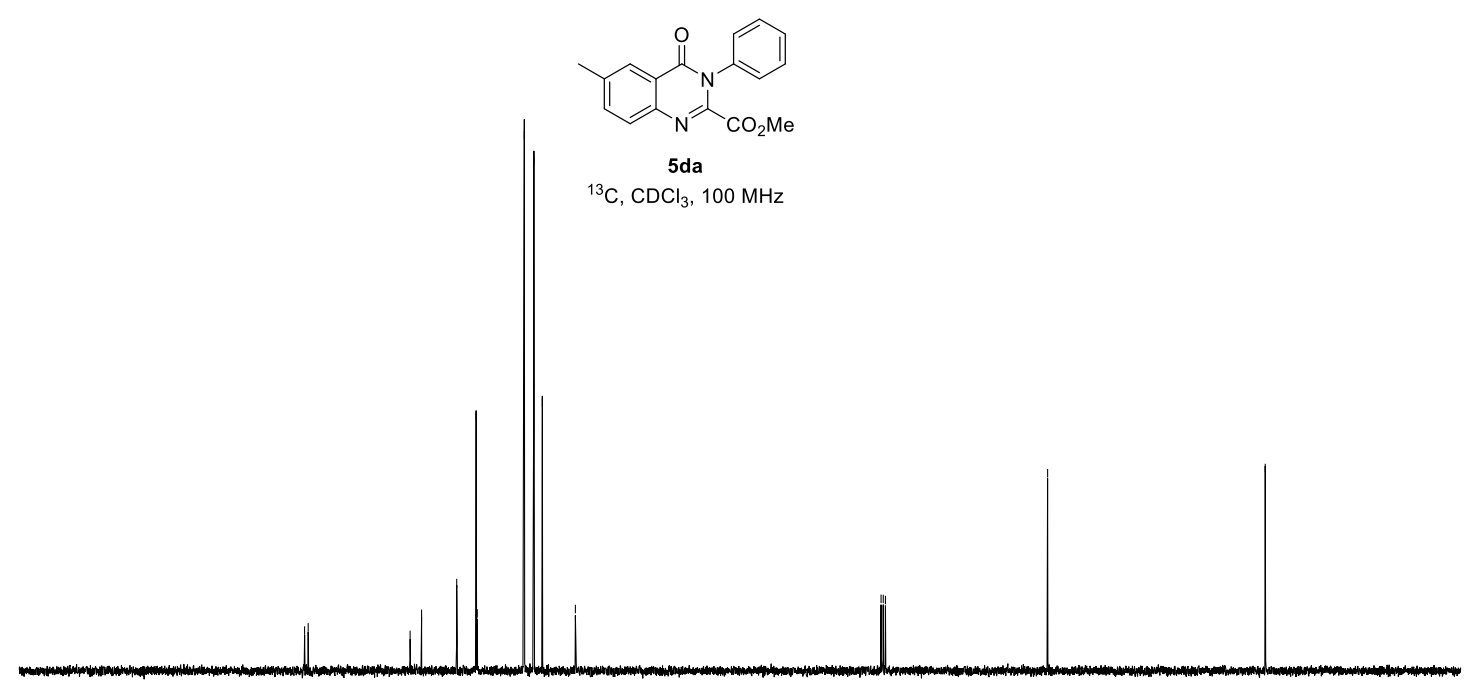

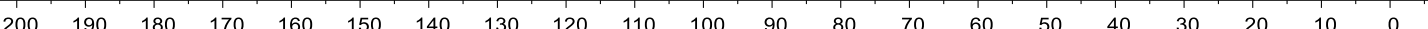




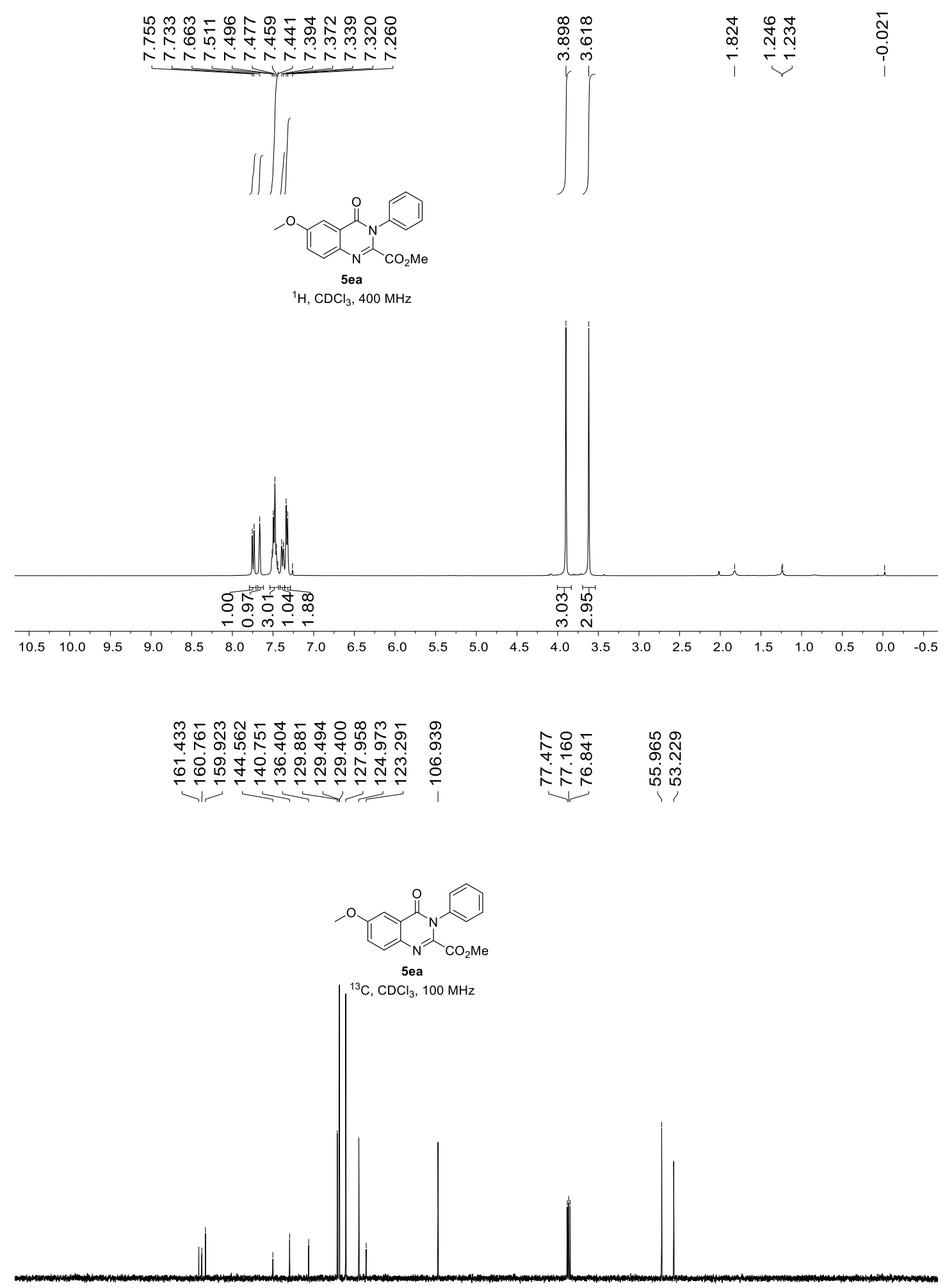

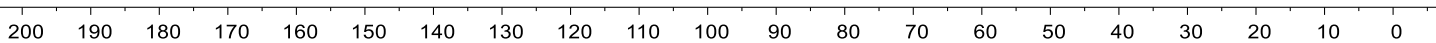




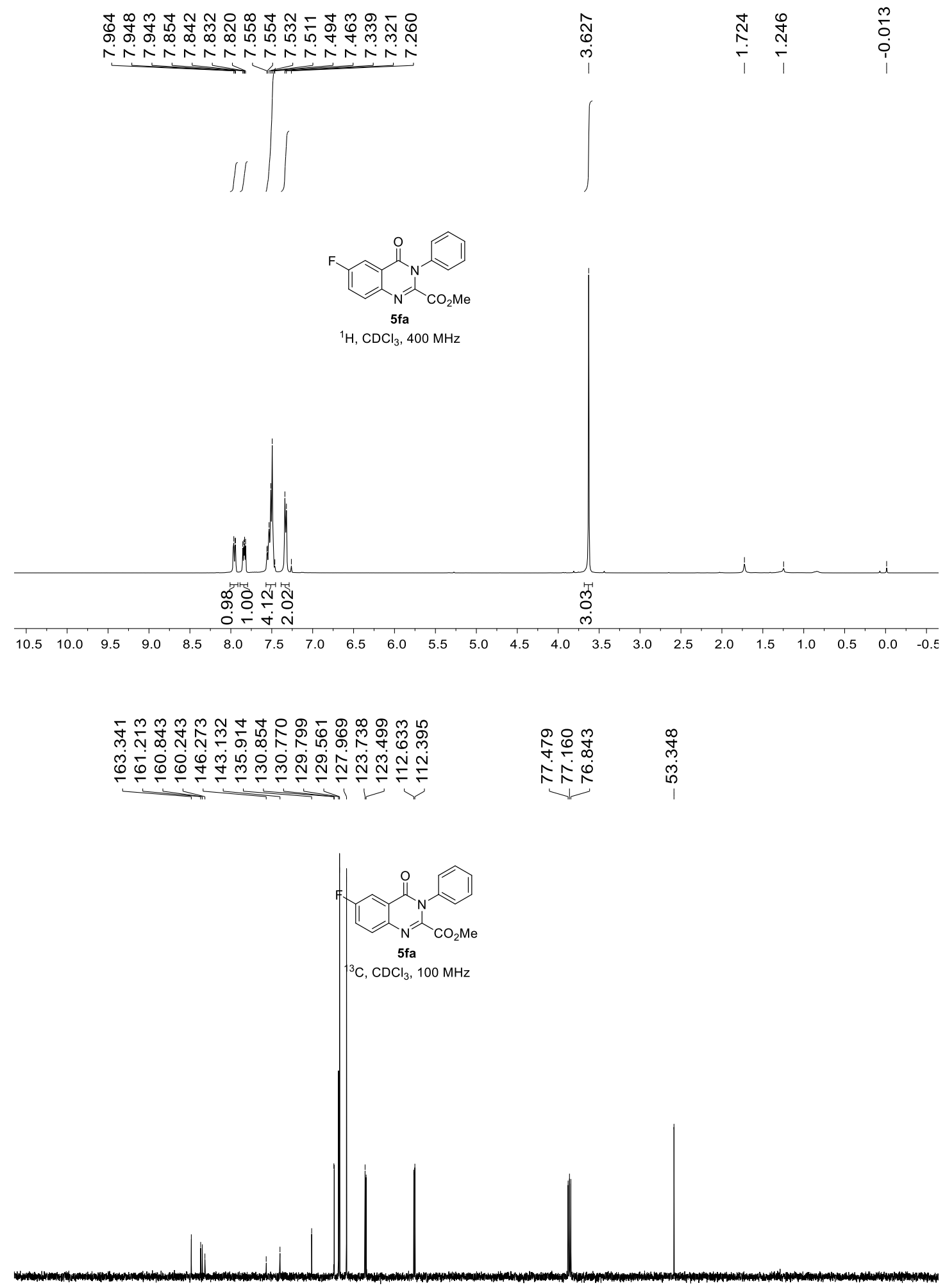

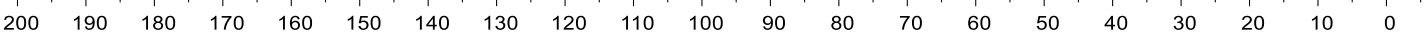




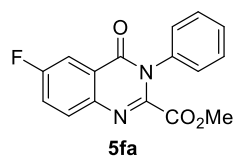

${ }^{19} \mathrm{~F}, \mathrm{CDCl}_{3}, 376 \mathrm{MHz}$

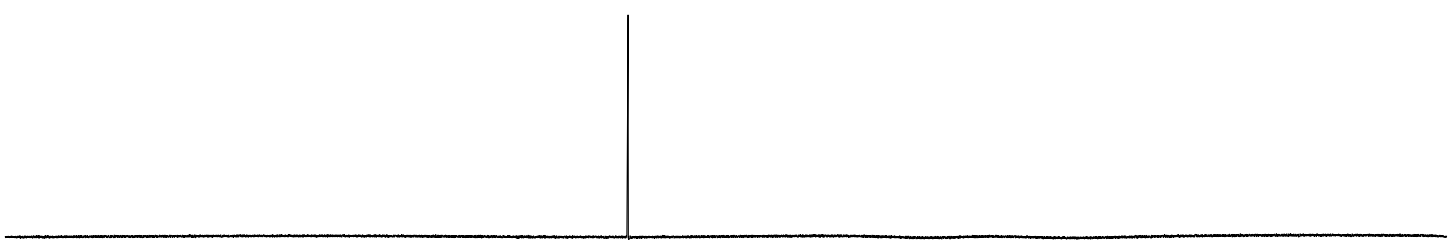

20

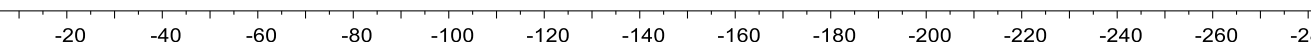



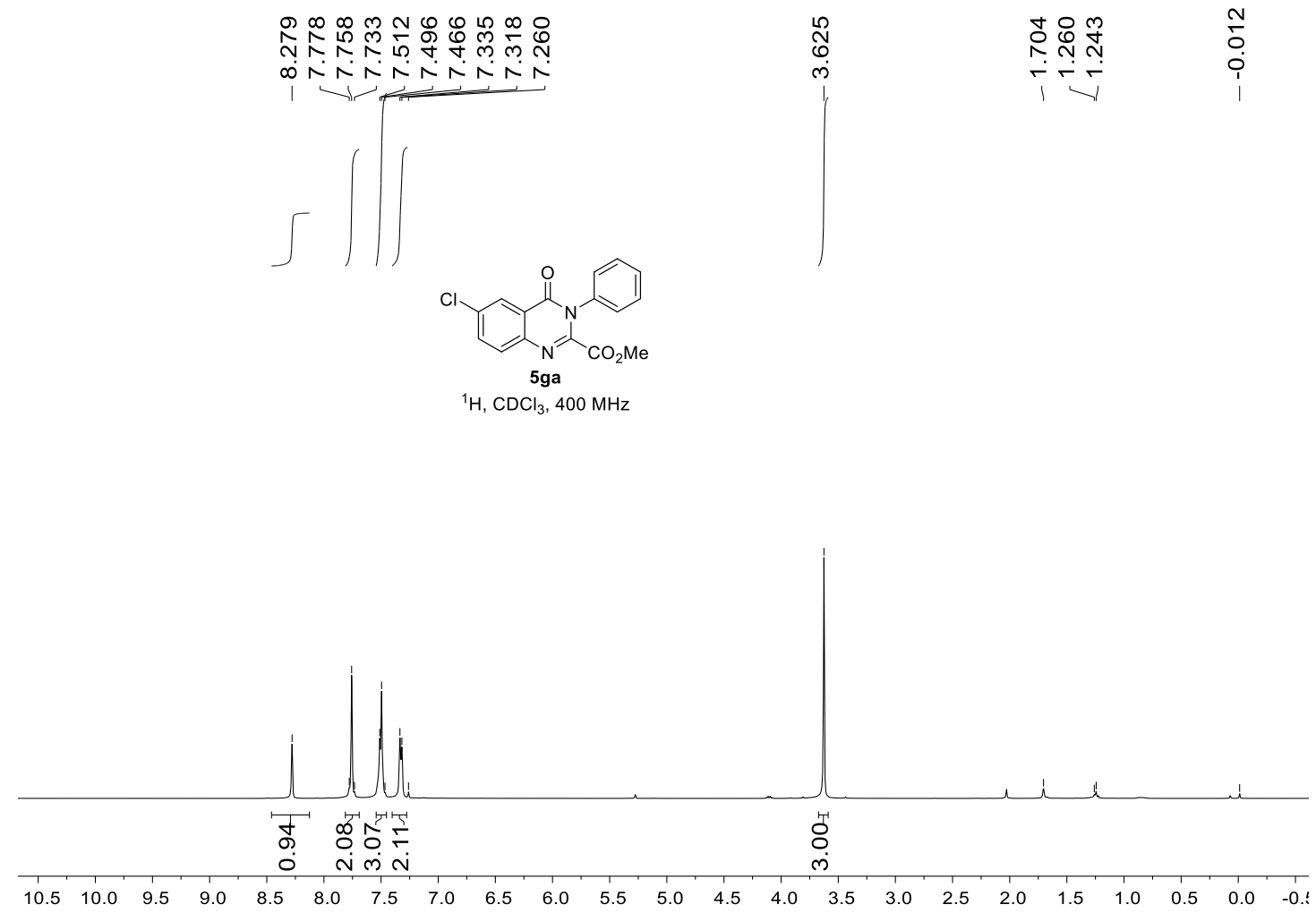

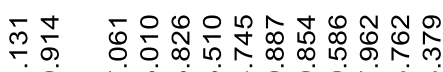

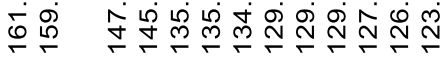

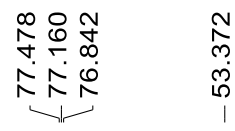

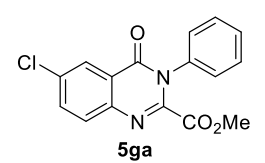

${ }^{13} \mathrm{C}, \mathrm{CDCl}_{3}, 100 \mathrm{MHz}$

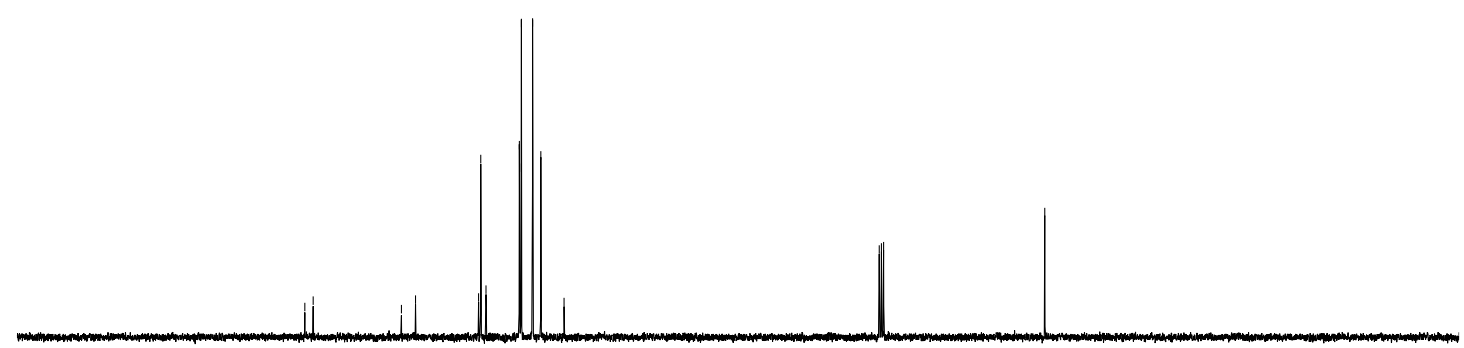

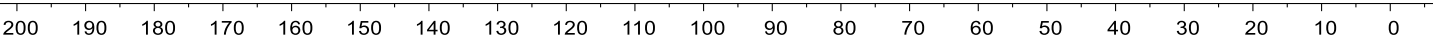




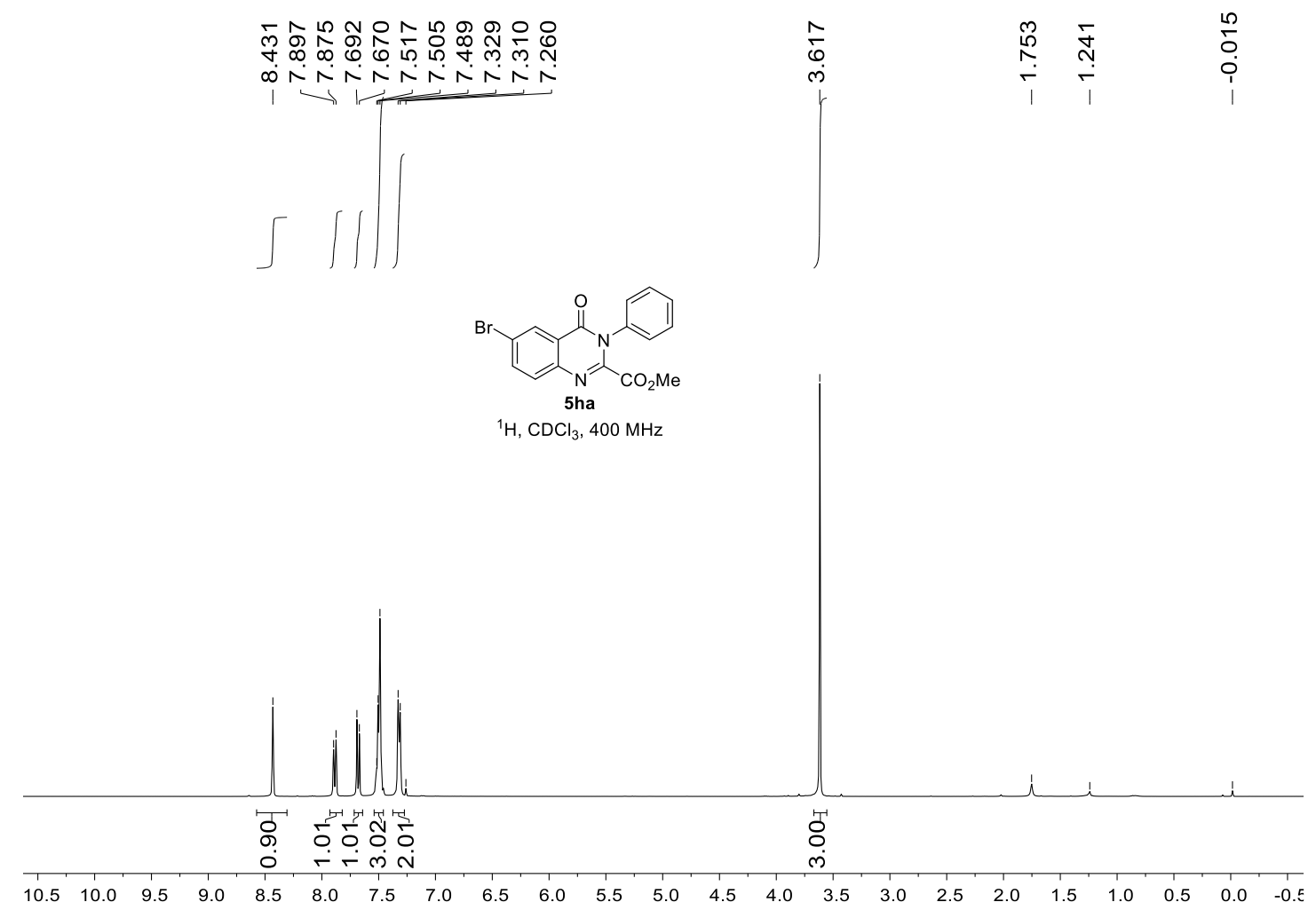

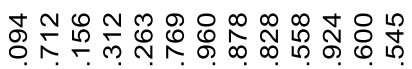

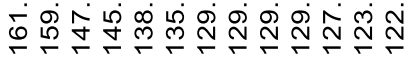

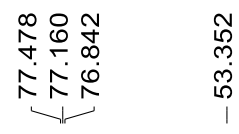

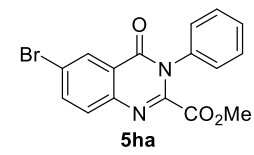

${ }^{13} \mathrm{C}, \mathrm{CDCl}_{3}, 100 \mathrm{MHz}$

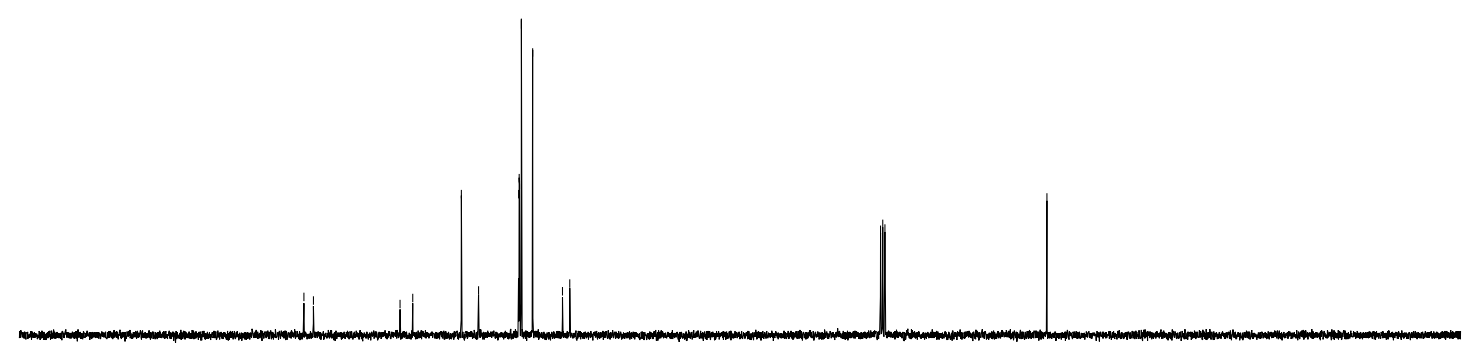

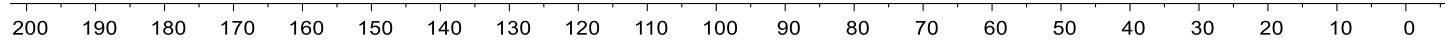




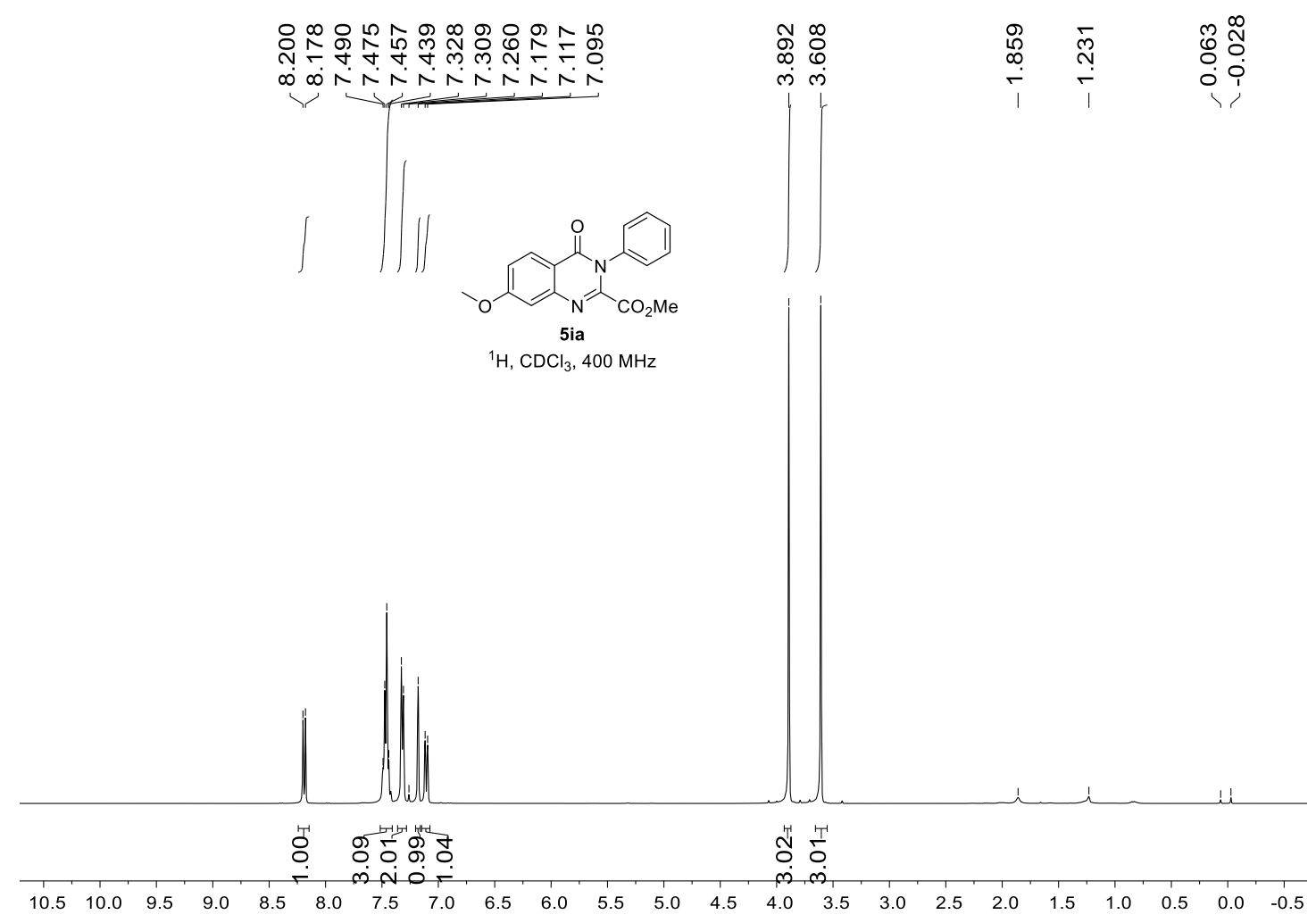

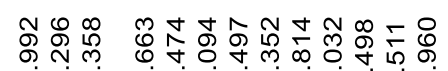

广்:

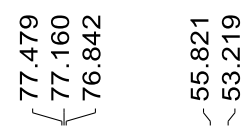

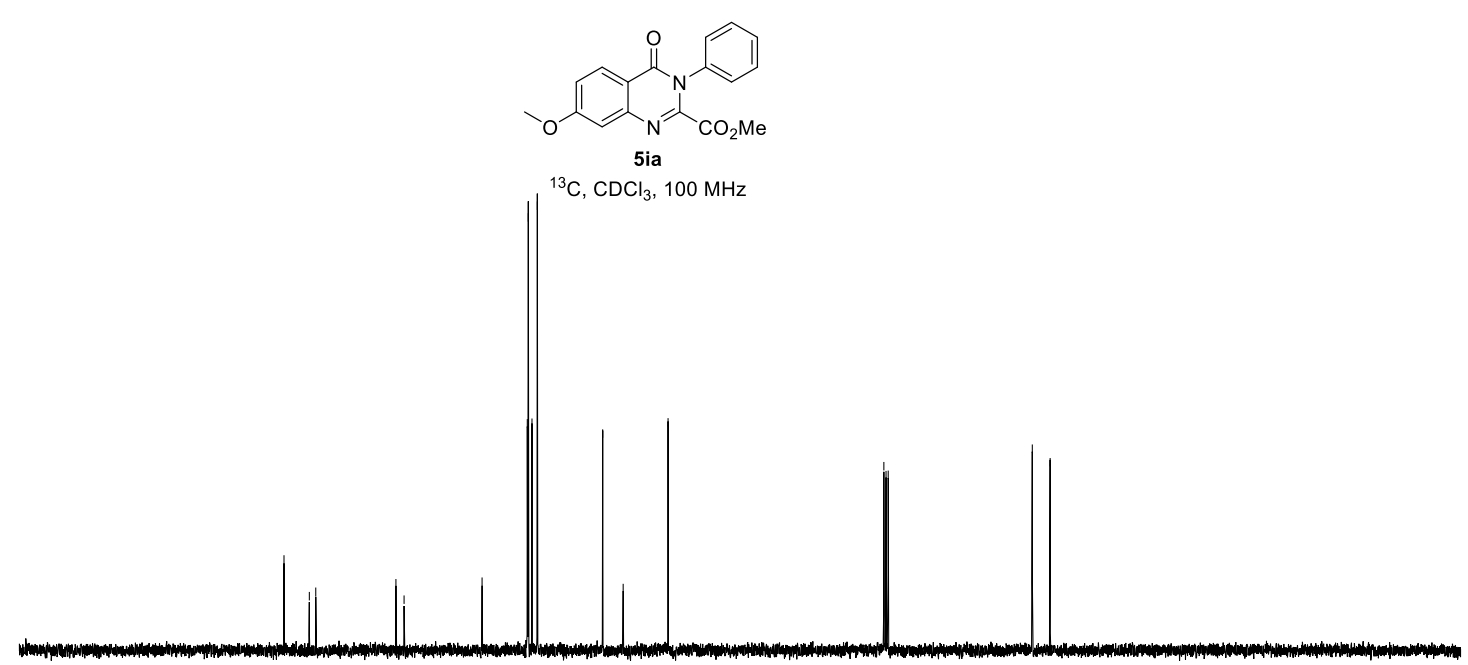

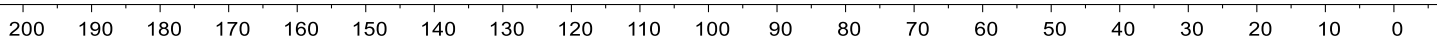




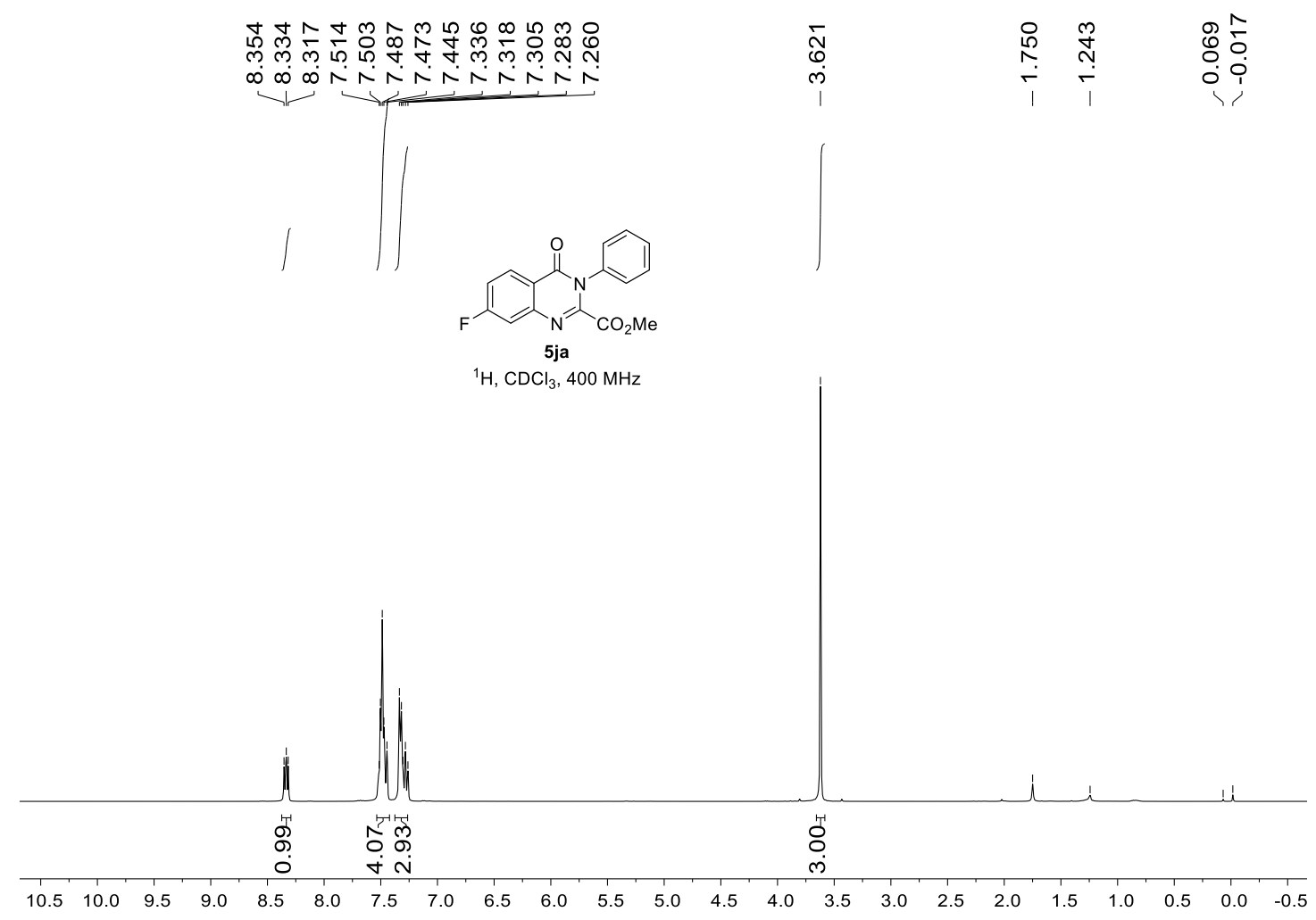

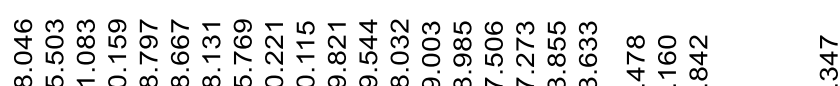

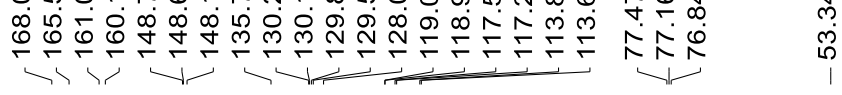

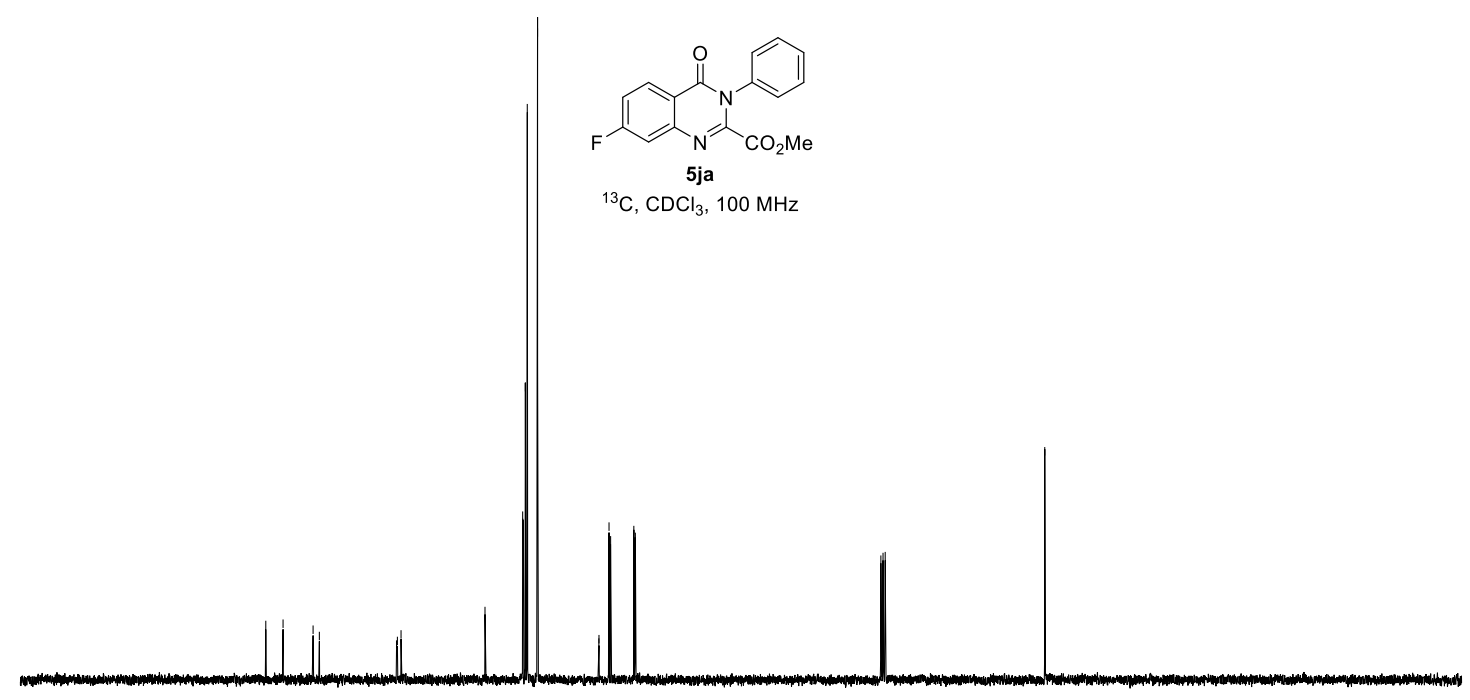

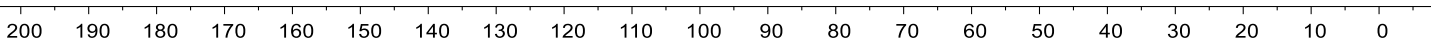



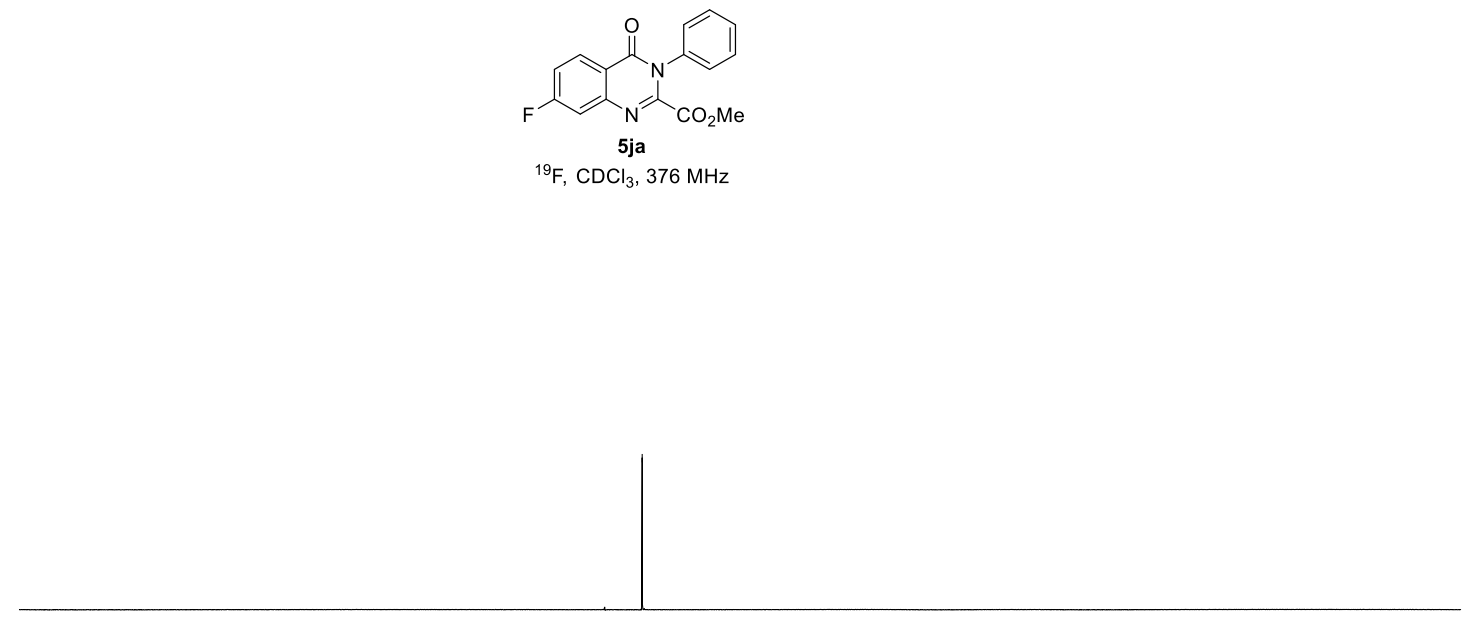

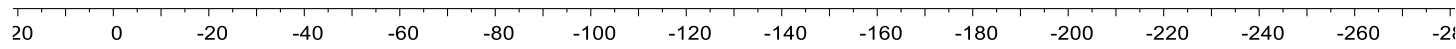




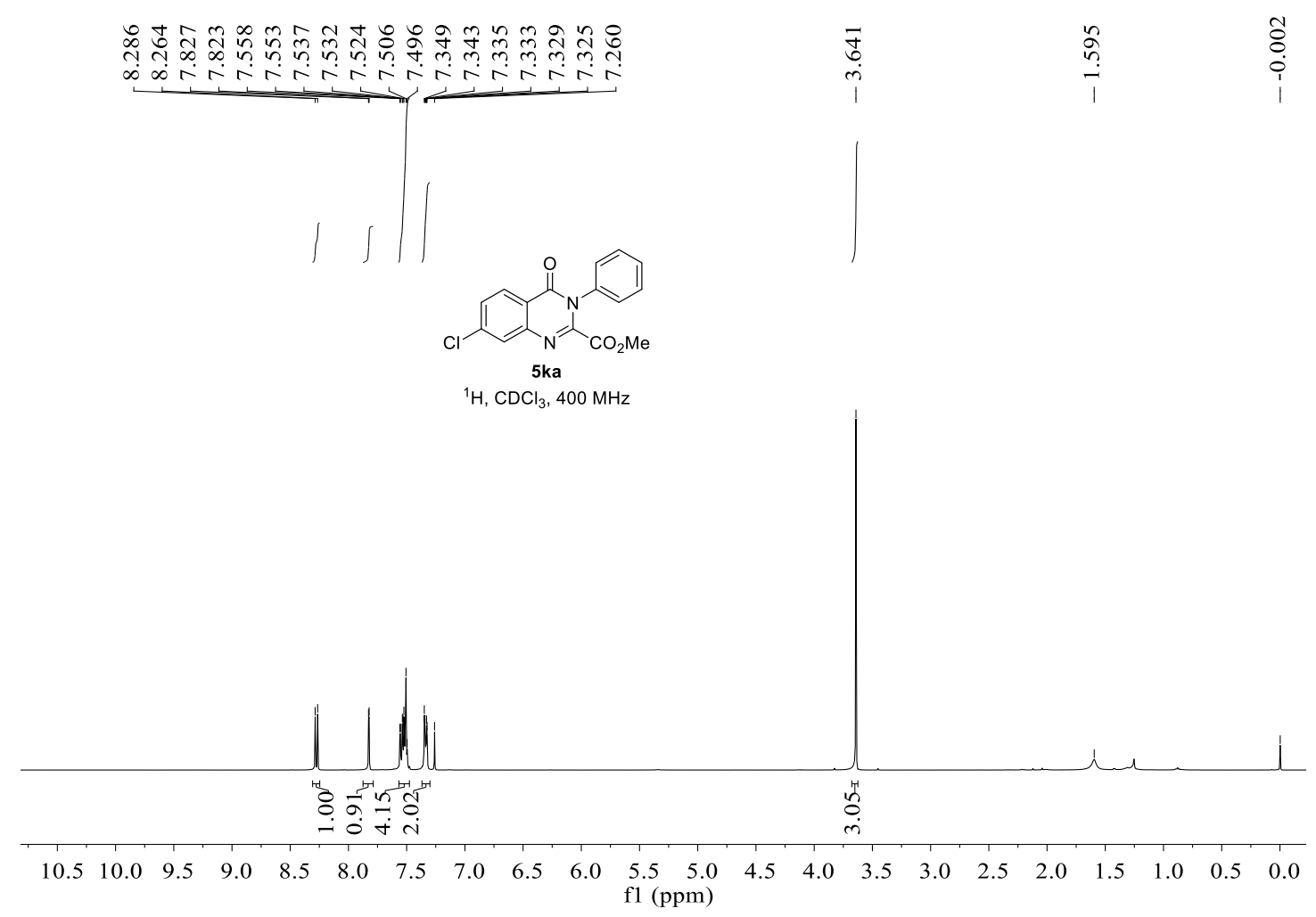

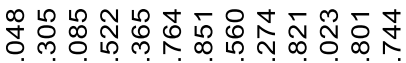

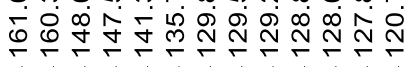

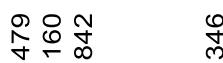

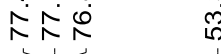

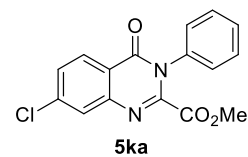

${ }^{13} \mathrm{C}, \mathrm{CDCl}_{3}, 100 \mathrm{MHz}$

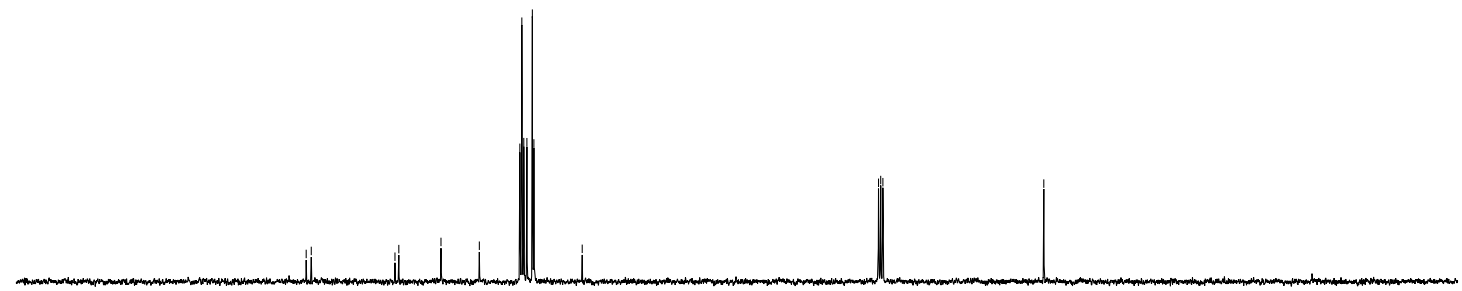

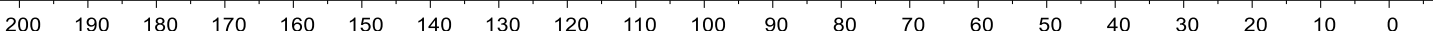



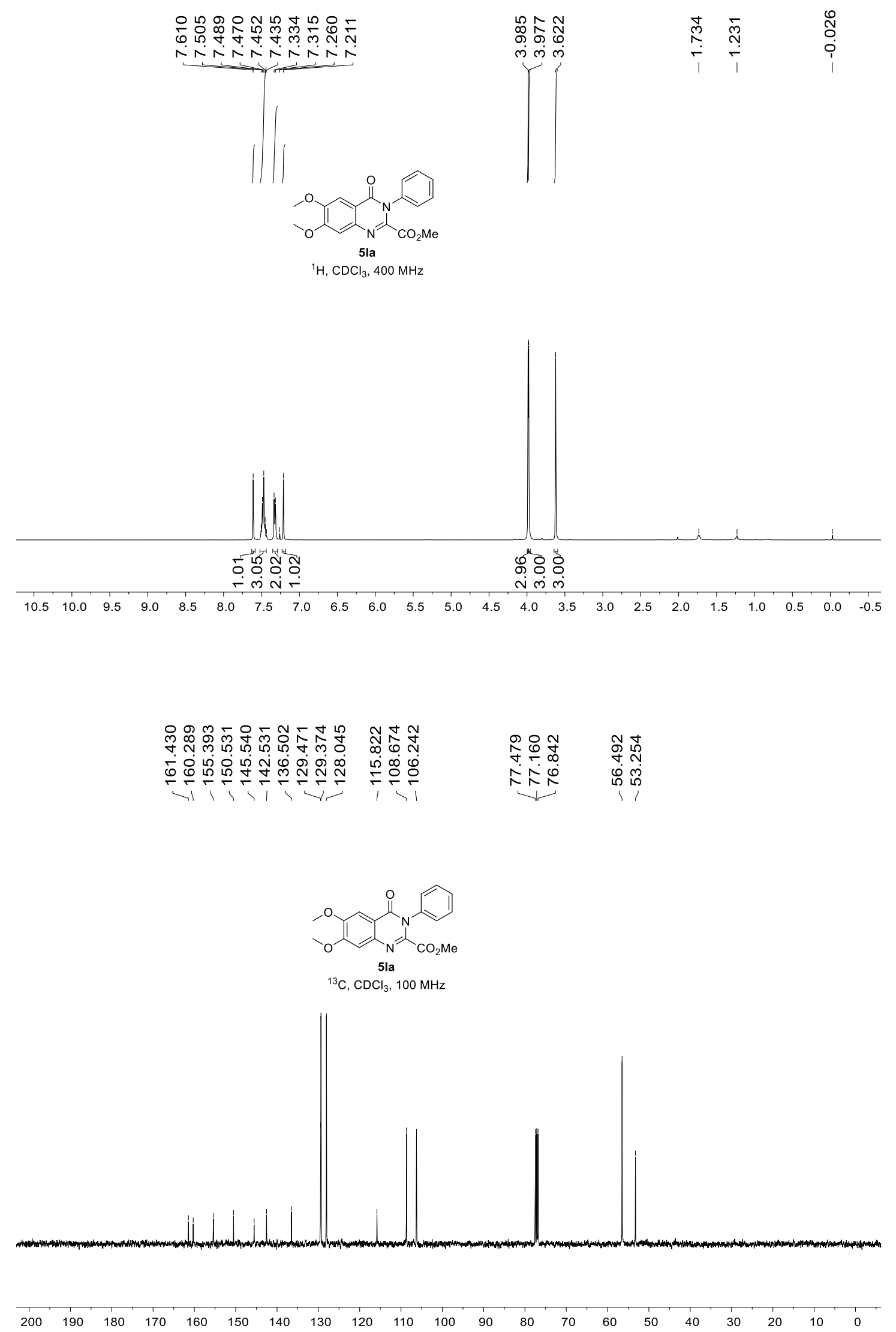


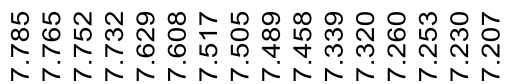

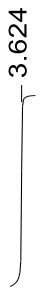

0
$\infty$
0
$\stackrel{-}{1}$

$\stackrel{0}{\circ}$

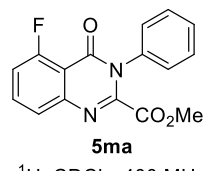

${ }^{1} \mathrm{H}, \mathrm{CDCl}_{3}, 400 \mathrm{MHz}$

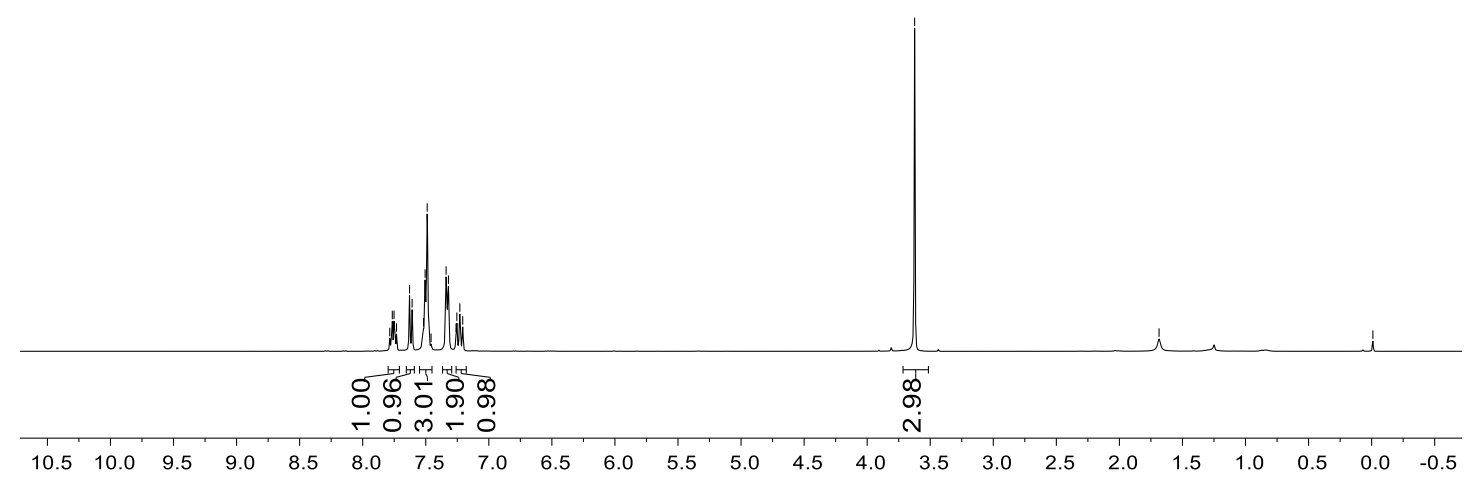

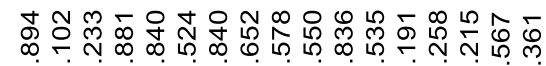

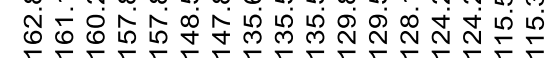

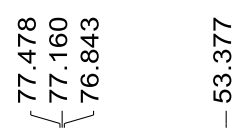

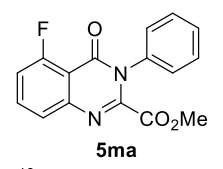

${ }^{13} \mathrm{C}, \mathrm{CDCl}_{3}, 100 \mathrm{MHz}$

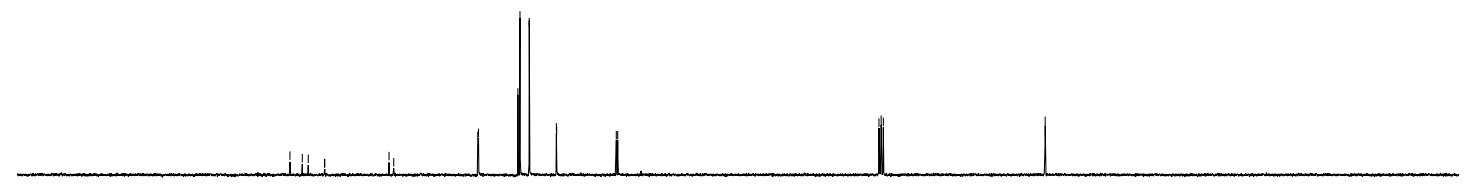

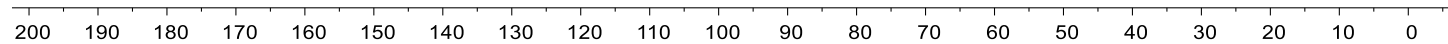




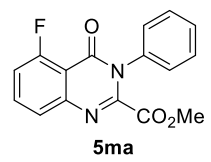

${ }^{19} \mathrm{~F}, \mathrm{CDCl}_{3}, 376 \mathrm{MHz}$

$\begin{array}{lllllllllllllllllllllll}-30 & -40 & -50 & -60 & -70 & -80 & -90 & -100 & -110 & -120 & -130 & -140 & -150 & -160 & -170 & -180 & -190 & -200 & -210 & -220 & -230 & -240 & -250\end{array}$ 


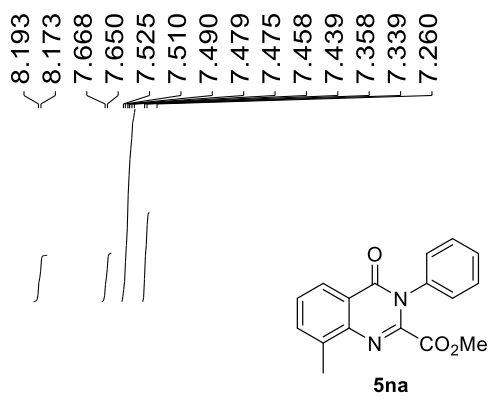

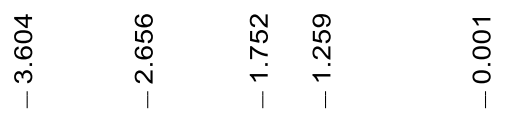

${ }^{1} \mathrm{H}, \mathrm{CDCl}_{3}, 400 \mathrm{MHz}$

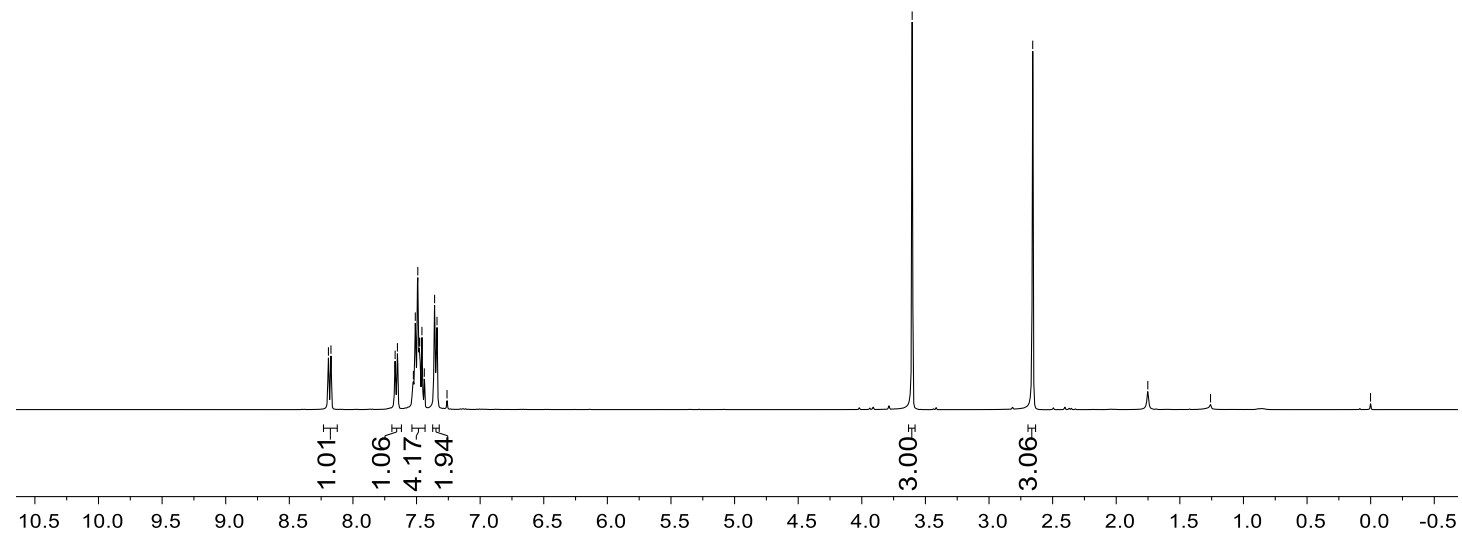

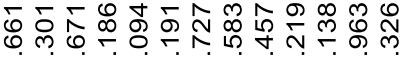

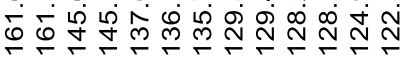

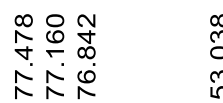

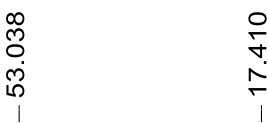

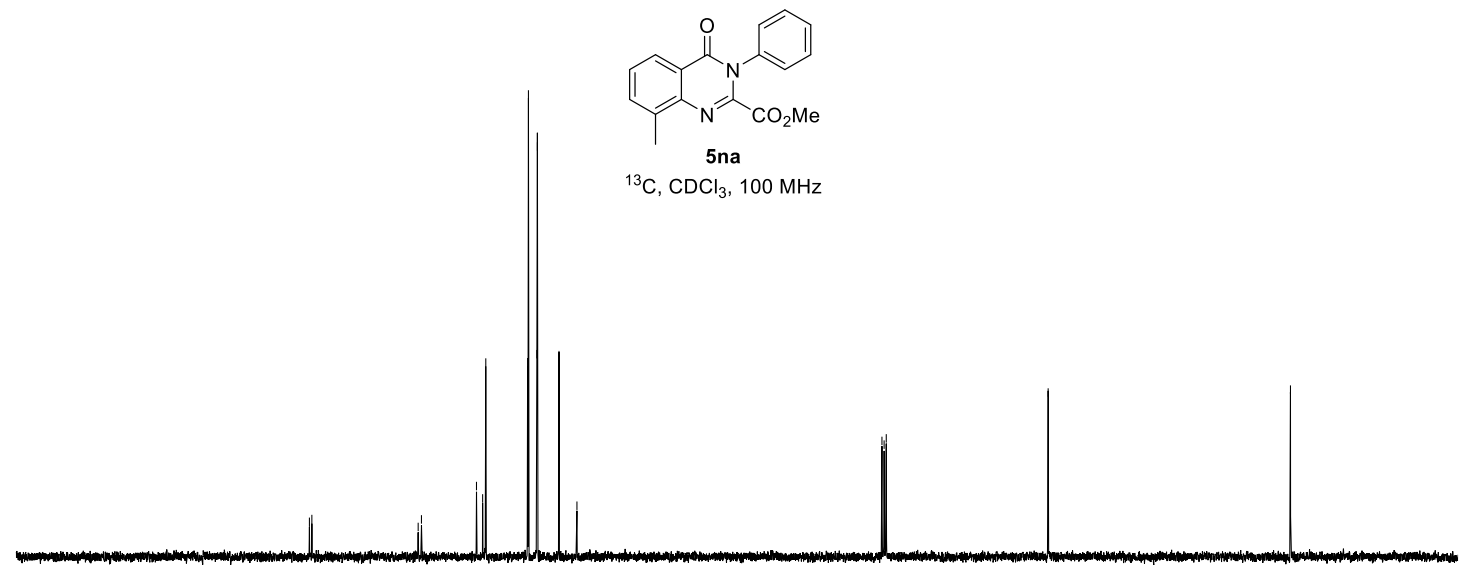

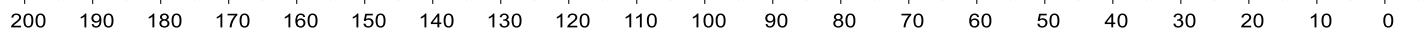




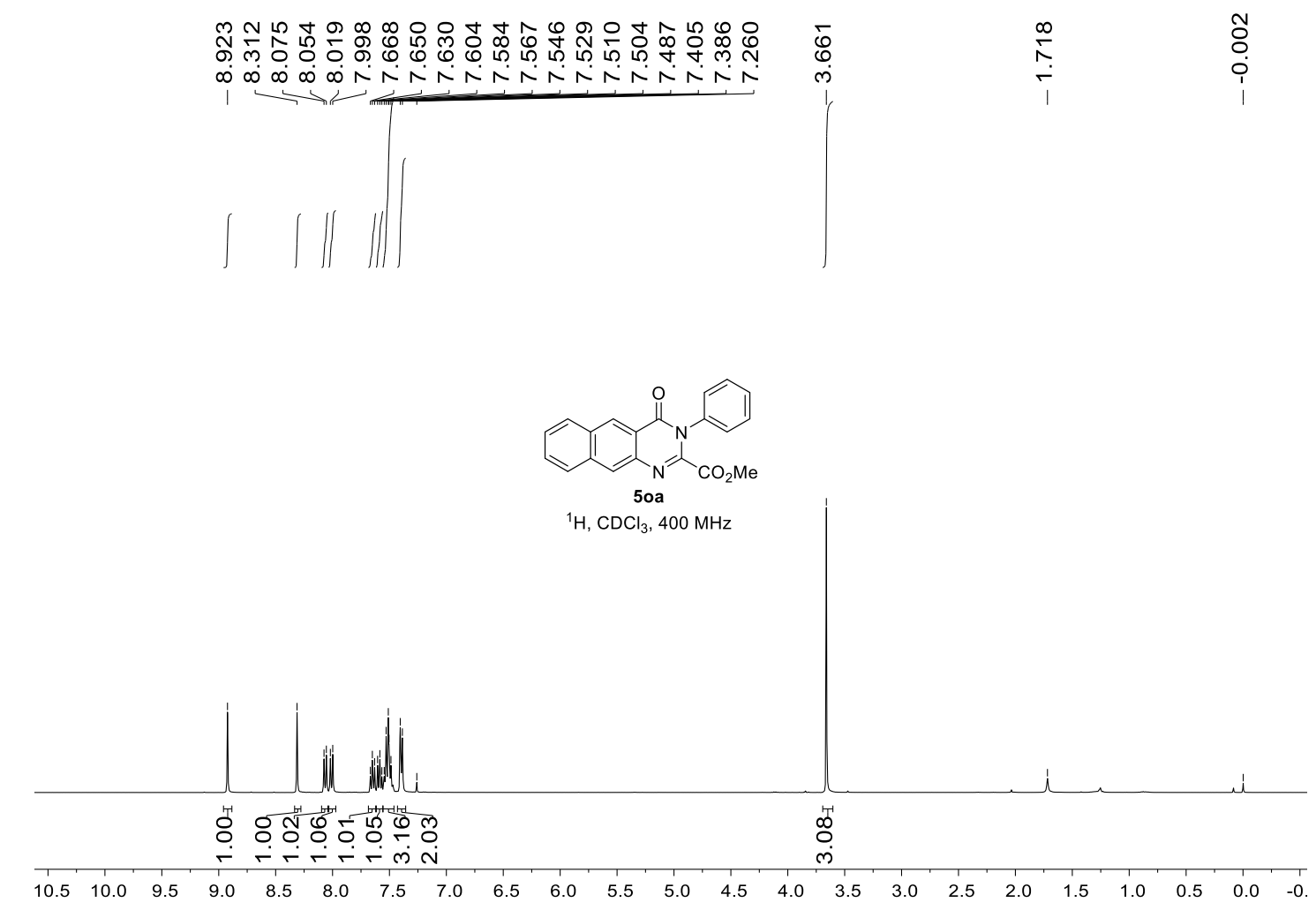

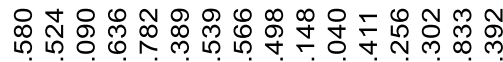

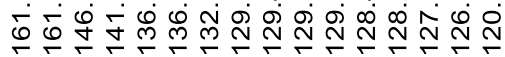

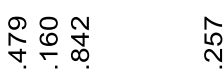

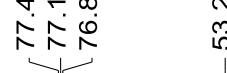

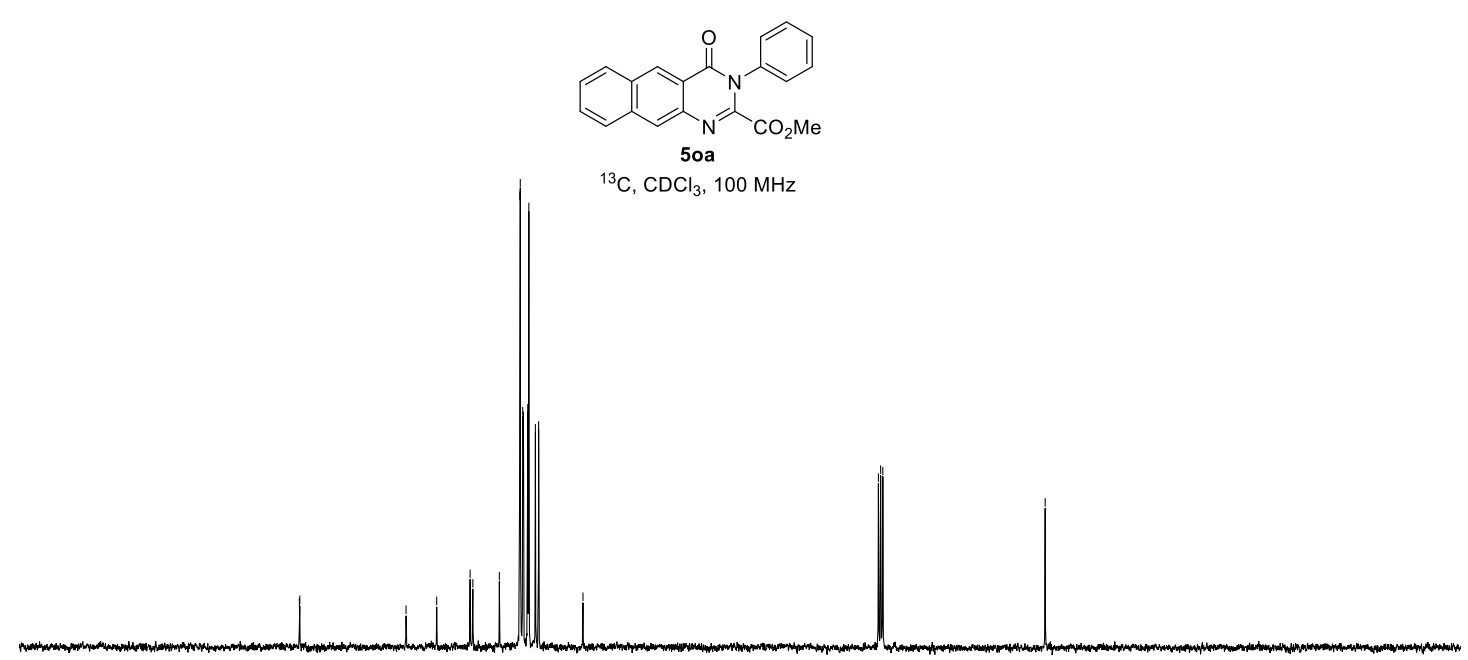

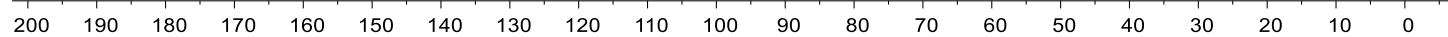



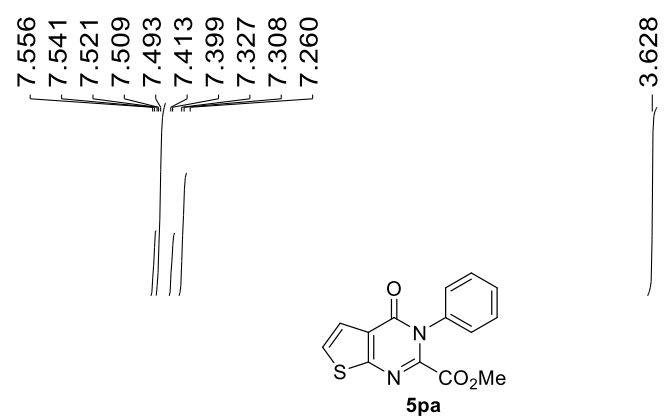

${ }^{1} \mathrm{H}, \mathrm{CDCl}_{3}, 400 \mathrm{MHz}$

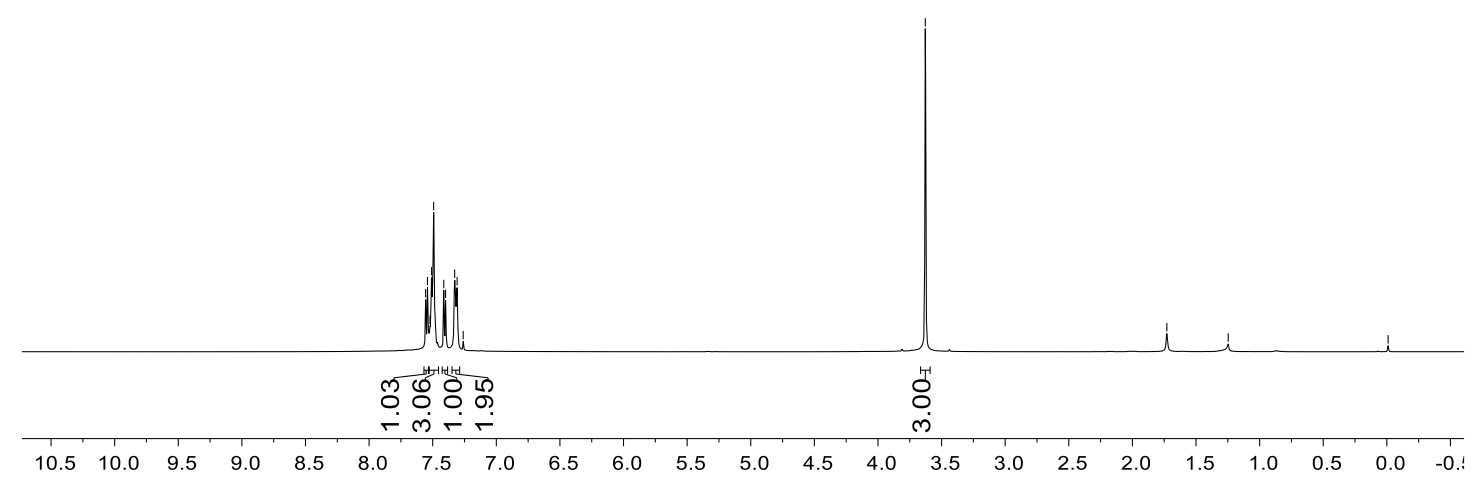

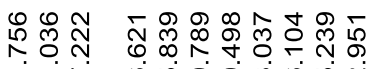

高它占

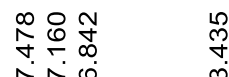

रN仓

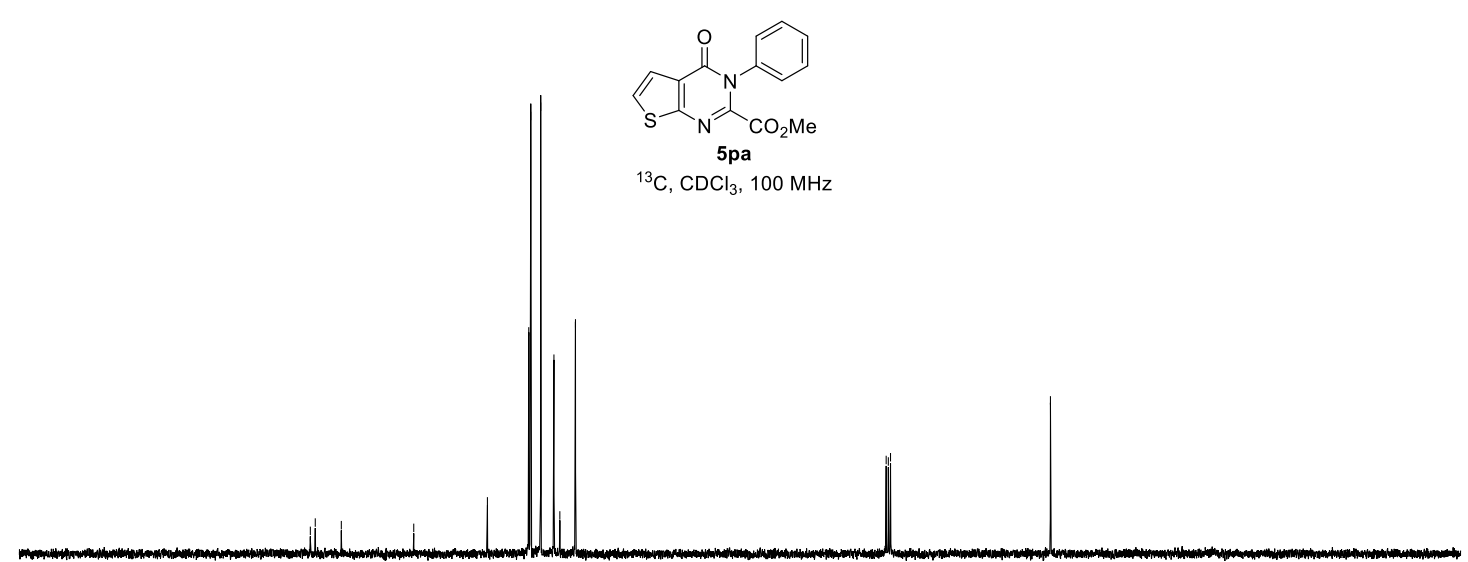

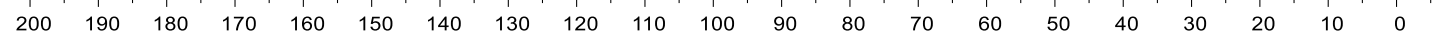



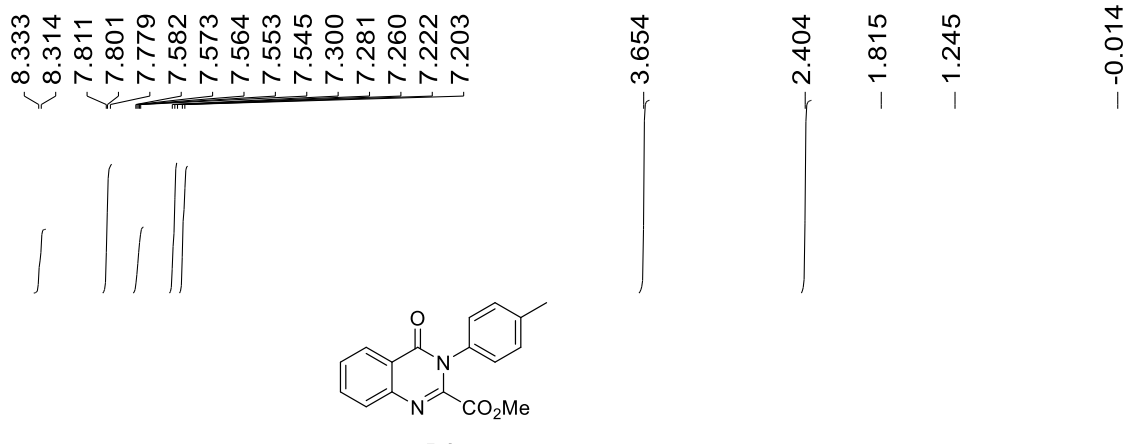

5 ab

${ }^{1} \mathrm{H}, \mathrm{CDCl}_{3}, 400 \mathrm{MHz}$

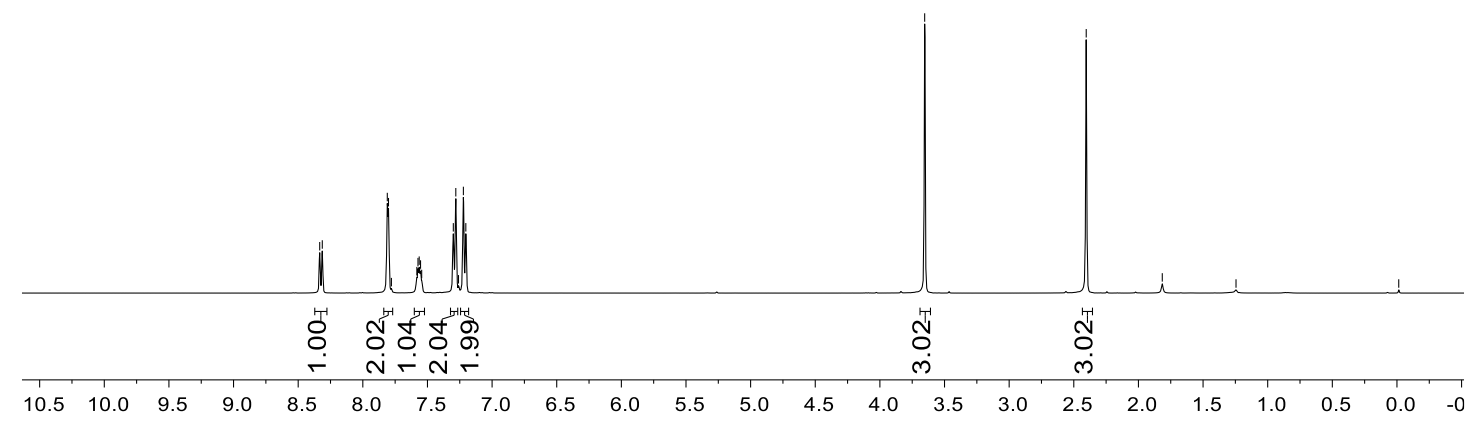

\&

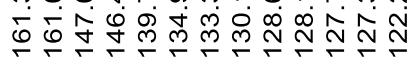

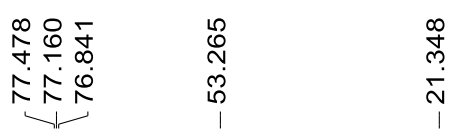

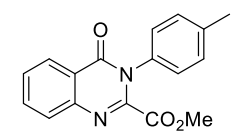

$5 \mathbf{a b}$

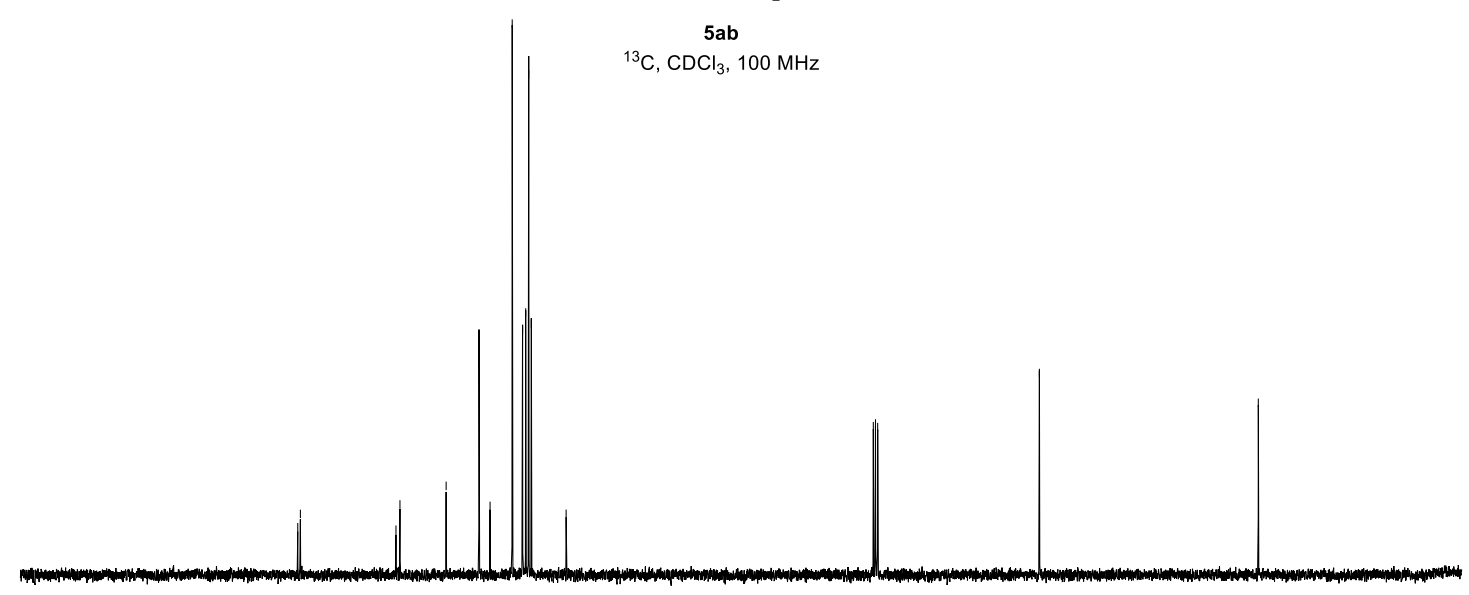

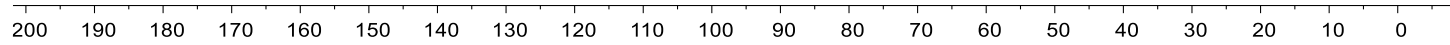



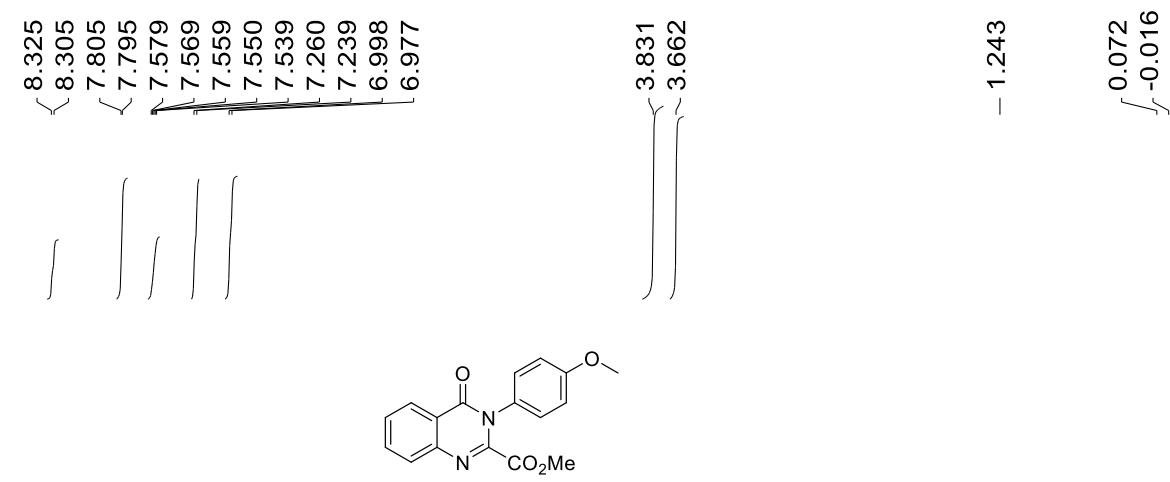

5 ac

${ }^{1} \mathrm{H}, \mathrm{CDCl}_{3}, 400 \mathrm{MHz}$
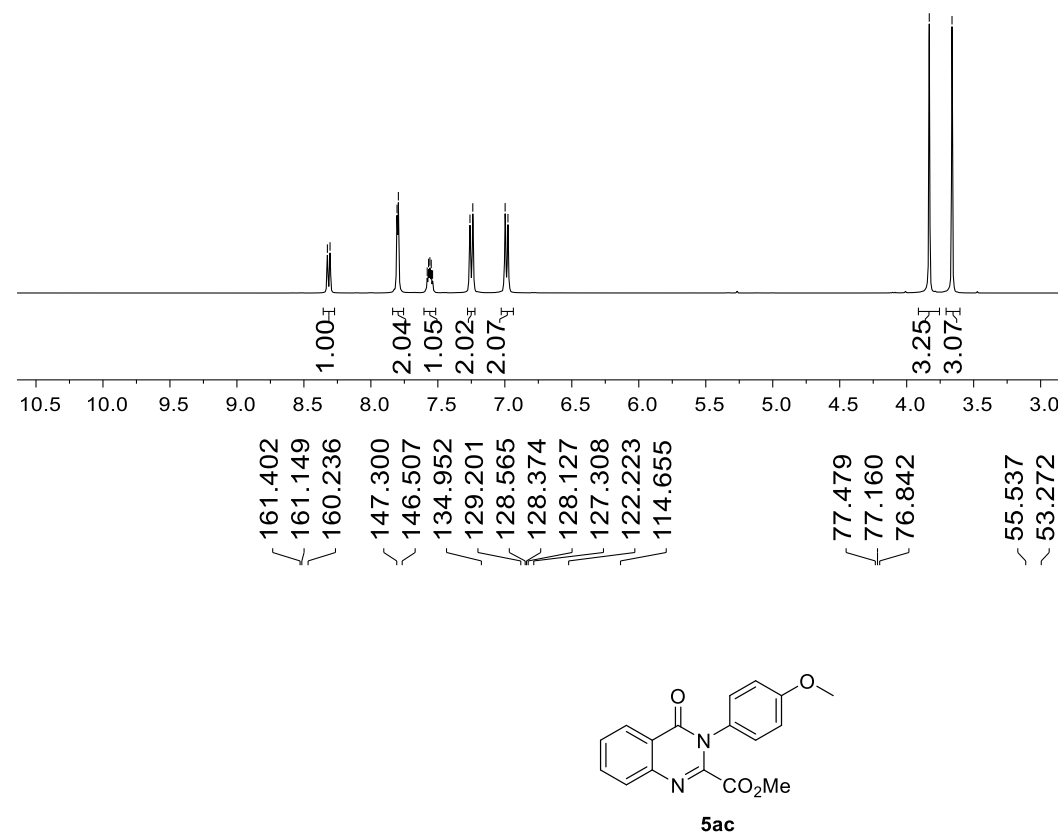

${ }^{13} \mathrm{C}, \mathrm{CDCl}_{3}, 100 \mathrm{MHz}$

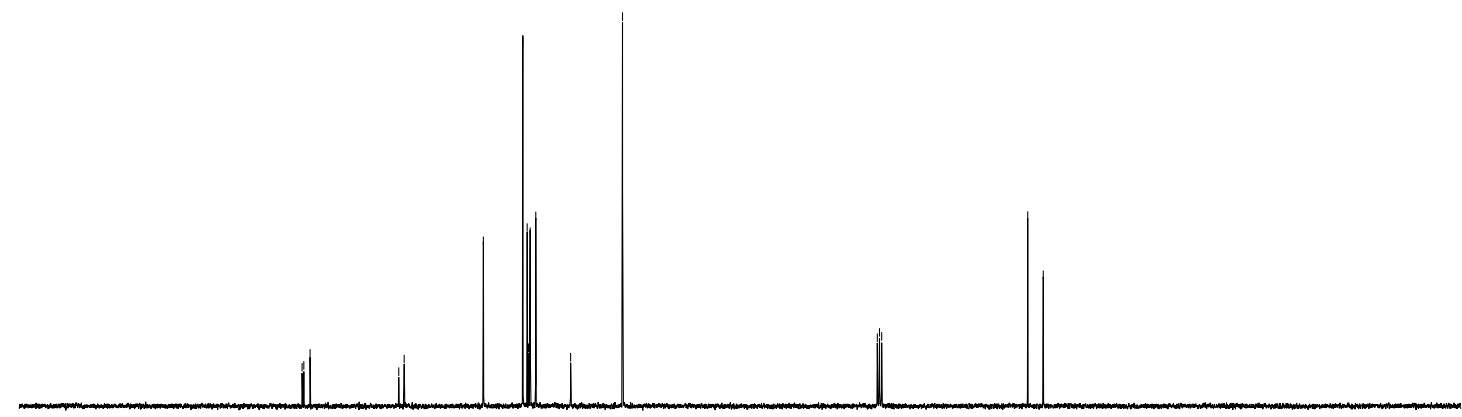

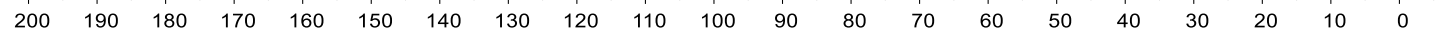




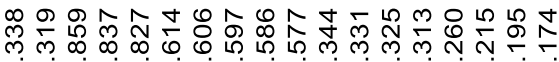

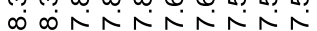
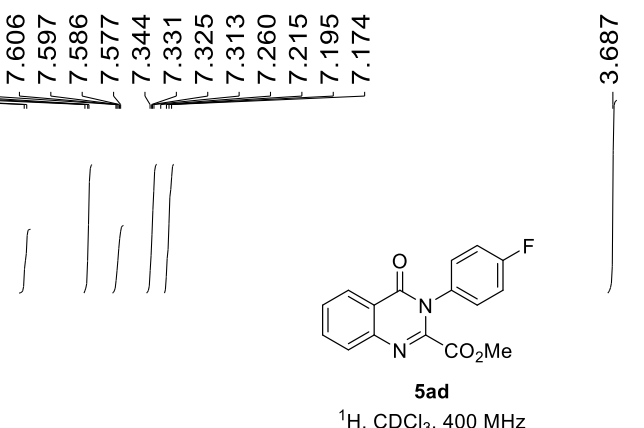

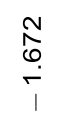

8
8
0
1

${ }^{1} \mathrm{H}, \mathrm{CDCl}_{3}, 400 \mathrm{MHz}$
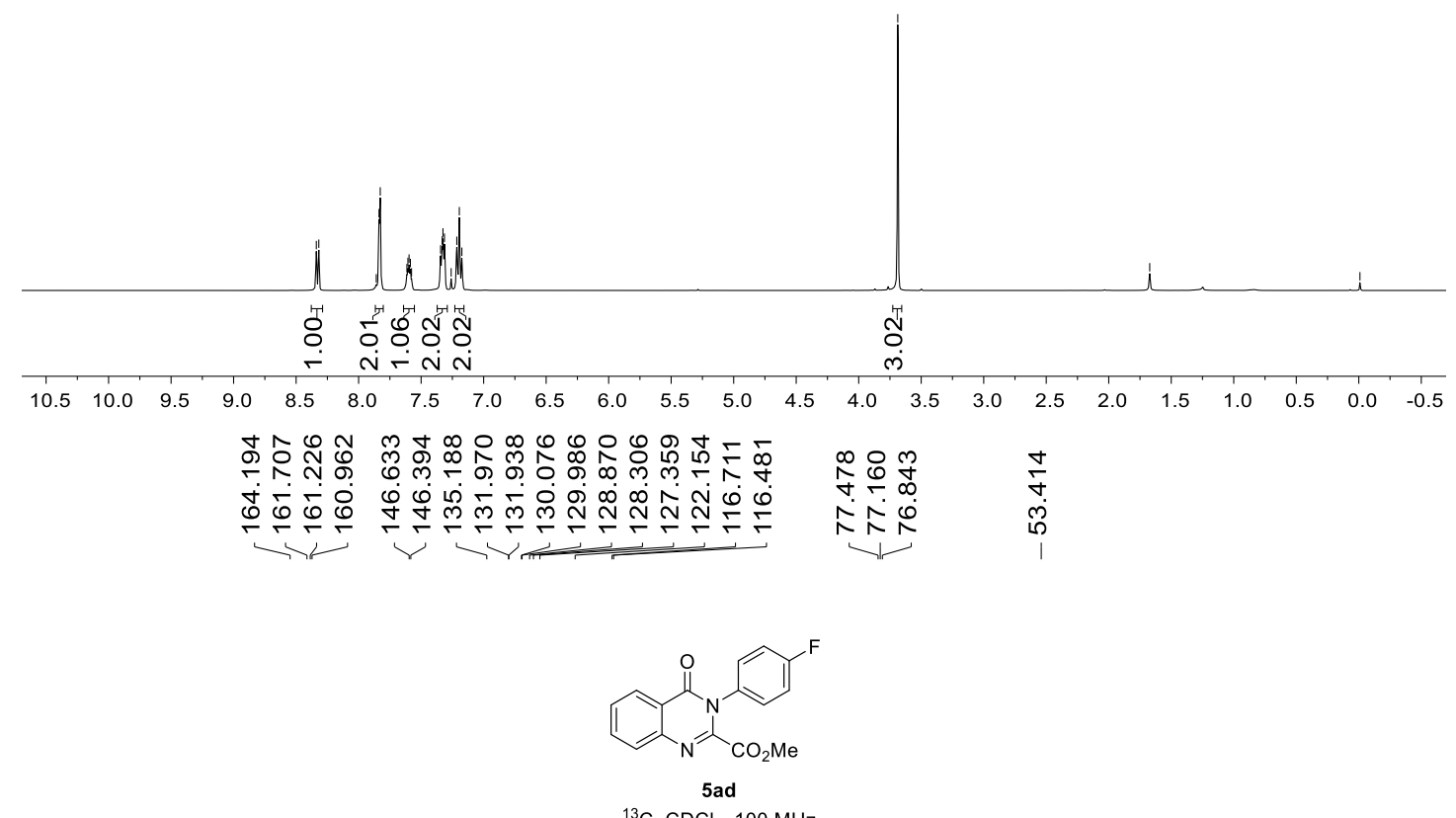

${ }^{13} \mathrm{C}, \mathrm{CDCl}_{3}, 100 \mathrm{MHz}$

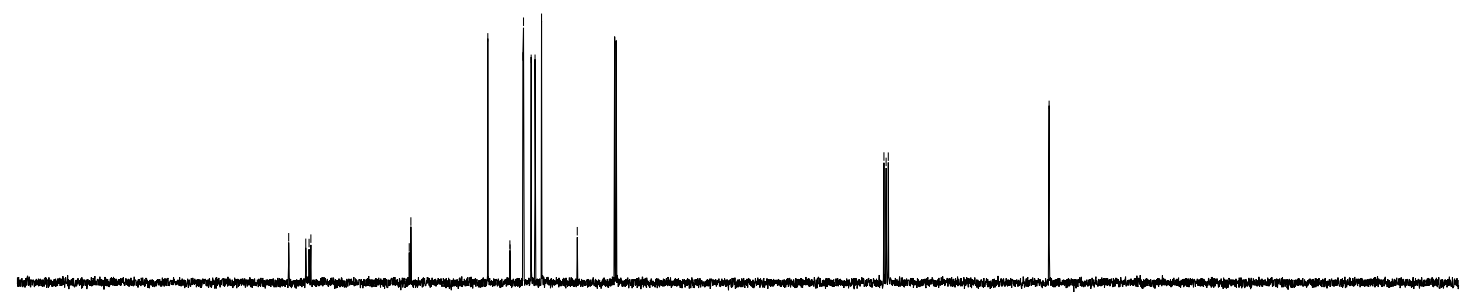

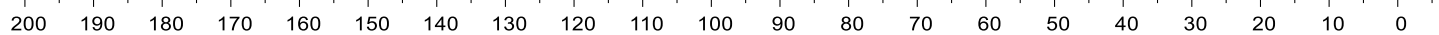




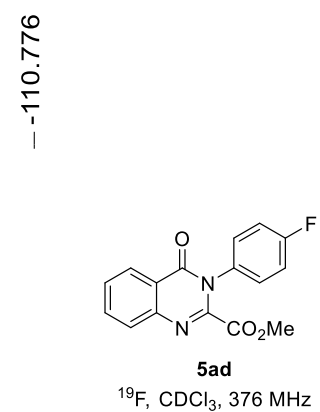
$\begin{array}{lllllllllllllllllllllllllllll}-30 & -40 & -50 & -60 & -70 & -80 & -90 & -100 & -110 & -120 & -130 & -140 & -150 & -160 & -170 & -180 & -190 & -200 & -210 & -220 & -230 & -240 & -250\end{array}$ 


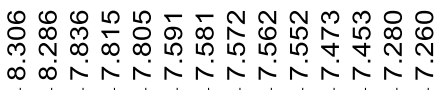

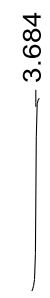

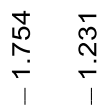

ָֻ
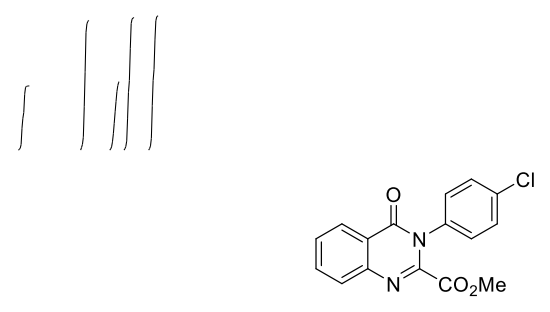

${ }^{1} \mathrm{H}, \mathrm{CDCl}_{3}, 400 \mathrm{MHz}$

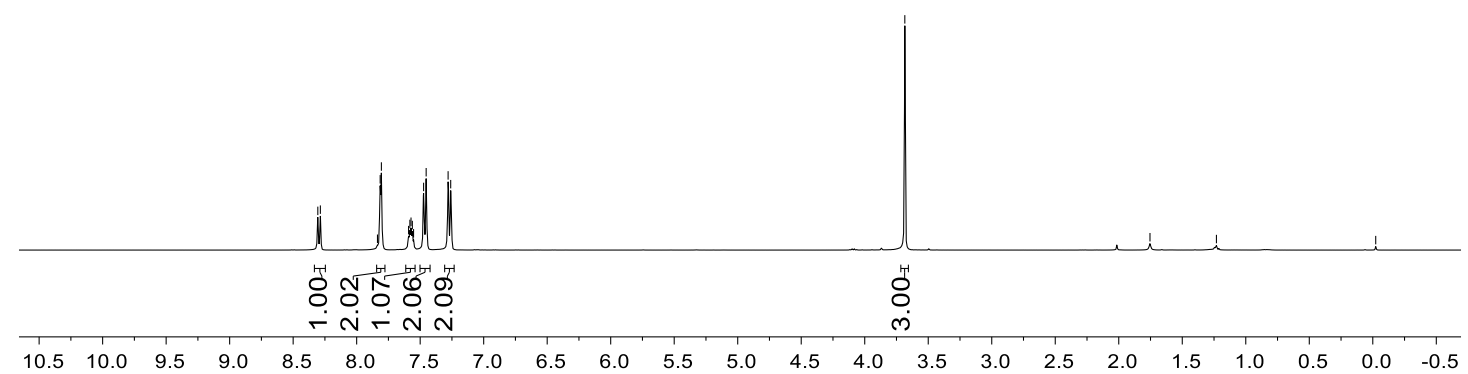

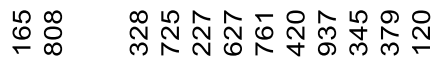

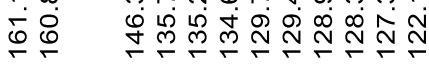

t.

ヘト゚

옴

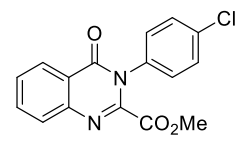

$5 a$

${ }^{13} \mathrm{C}, \mathrm{CDCl}_{3}, 100 \mathrm{MHz}$

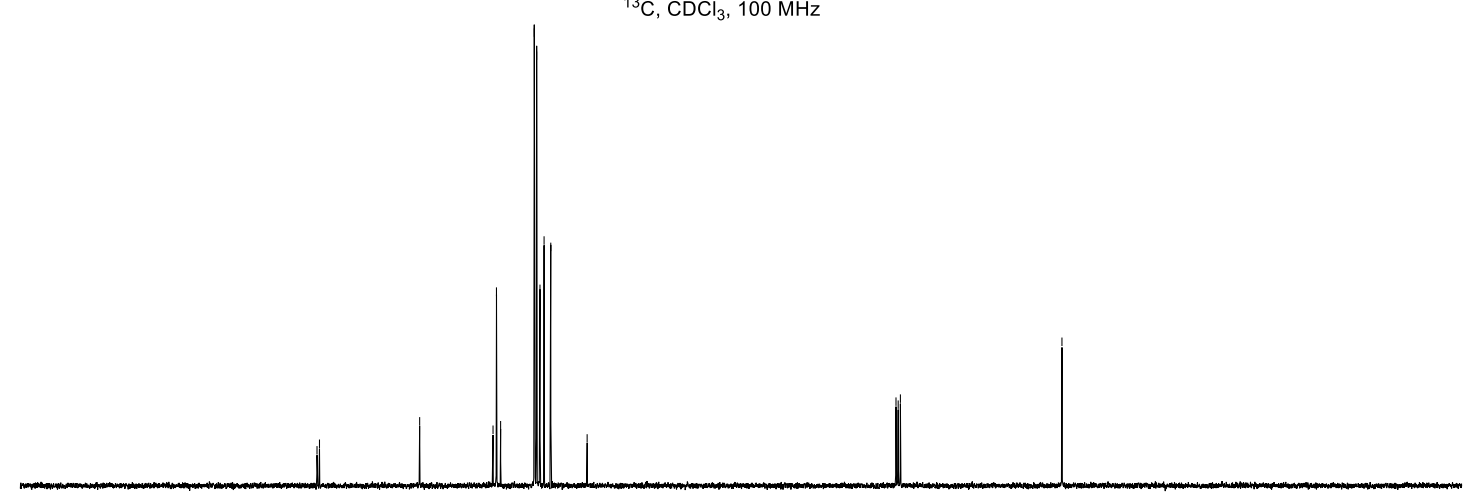

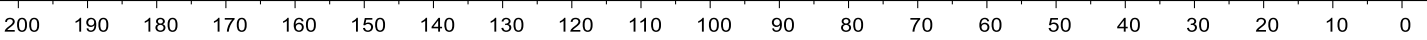




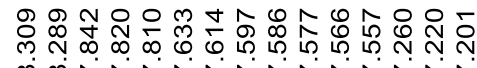

$\infty \infty \pi N+N \Lambda N$
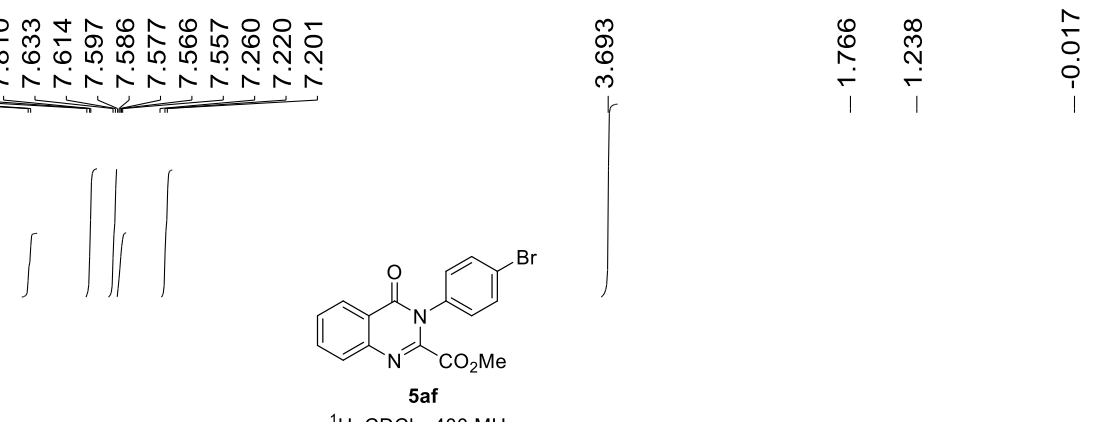

${ }^{1} \mathrm{H}, \mathrm{CDCl}_{3}, 400 \mathrm{MHz}$

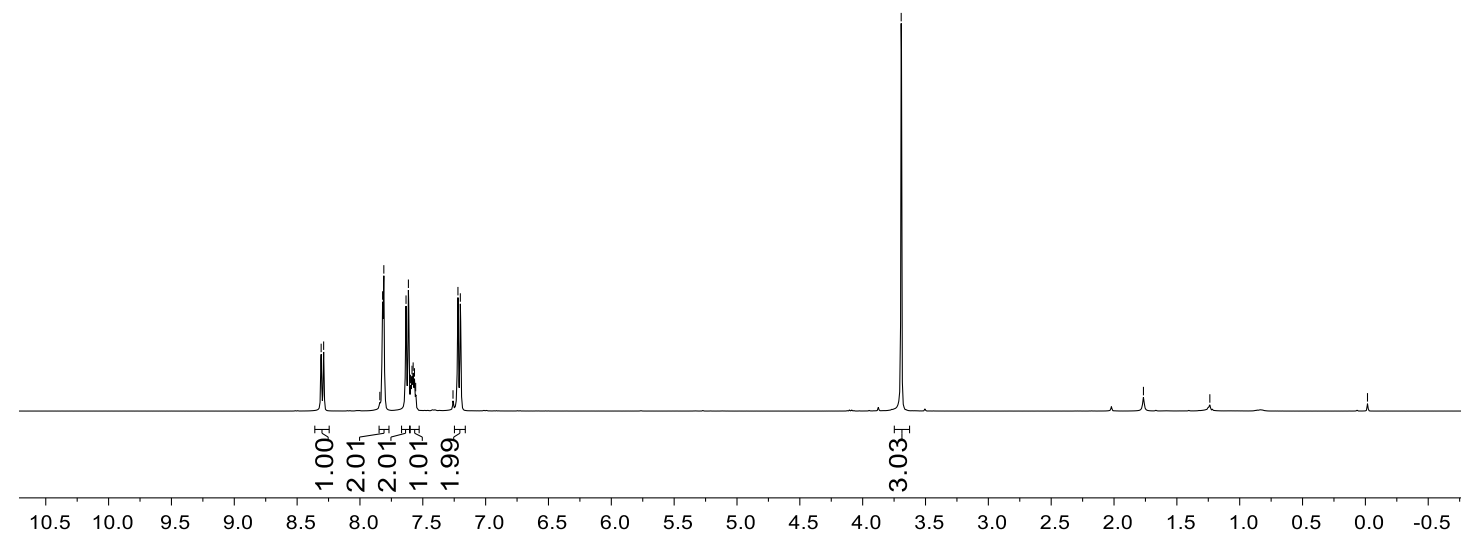

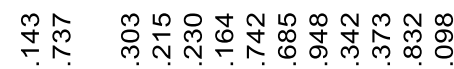

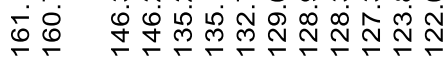

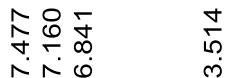

र亦

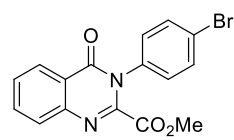

5 af

${ }^{13} \mathrm{C}, \mathrm{CDCl}_{3}, 100 \mathrm{MHz}$

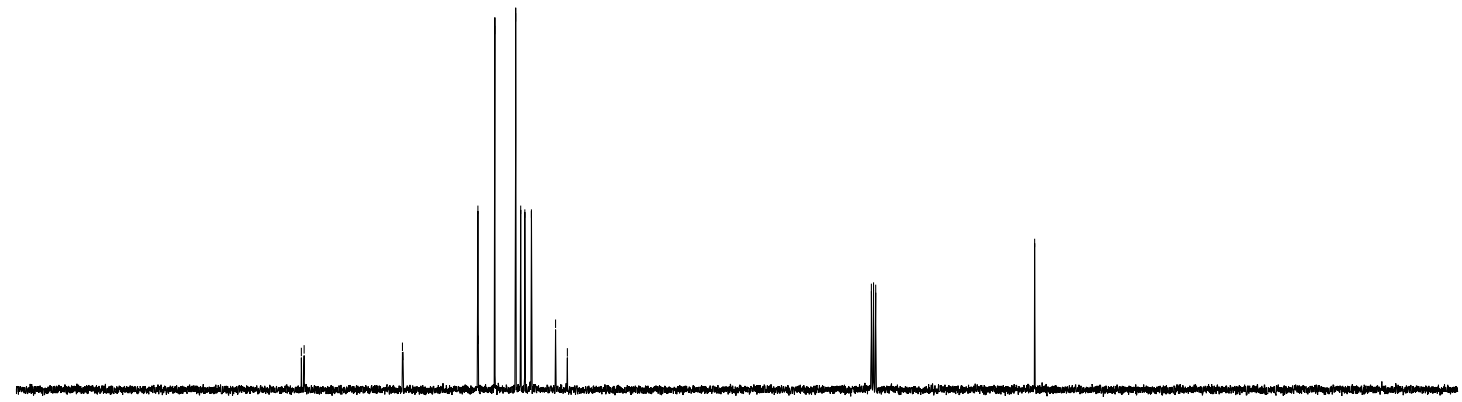

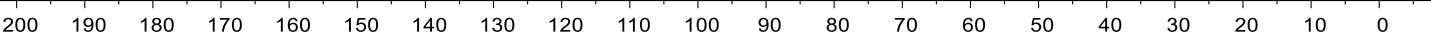




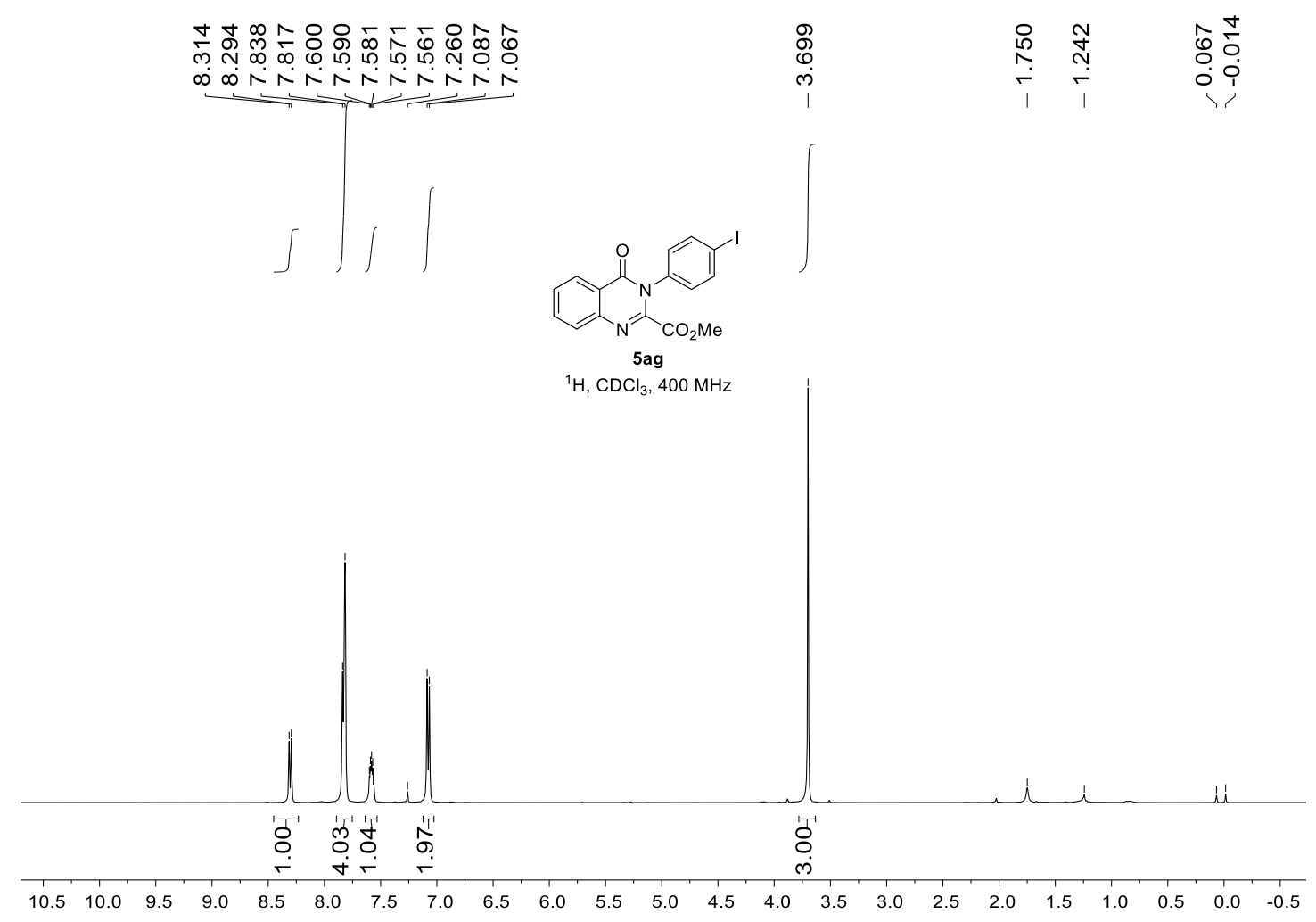

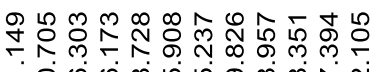

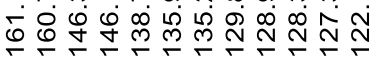

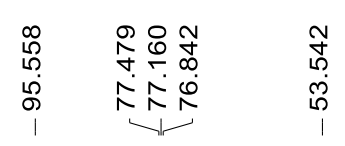

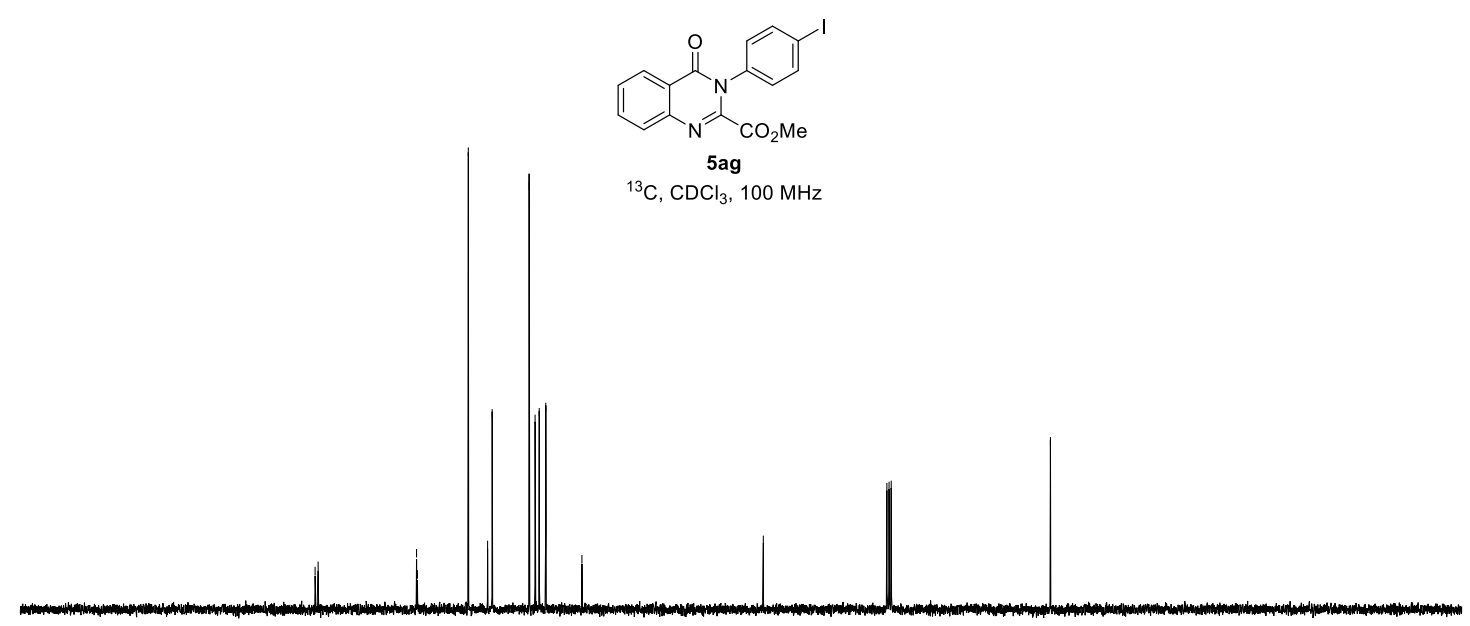

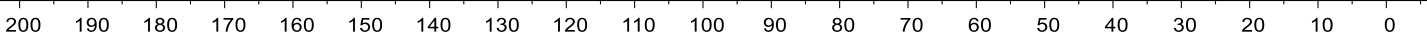




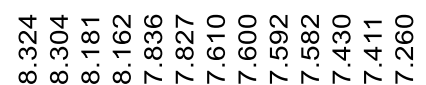

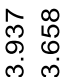

$\begin{array}{ll}\stackrel{\infty}{N} & \bar{N} \\ \stackrel{0}{\leftarrow} & 0 \\ 1 & i\end{array}$

$\int 11$

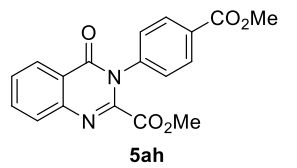

${ }^{1} \mathrm{H}, \mathrm{CDCl}_{3}, 400 \mathrm{MHz}$

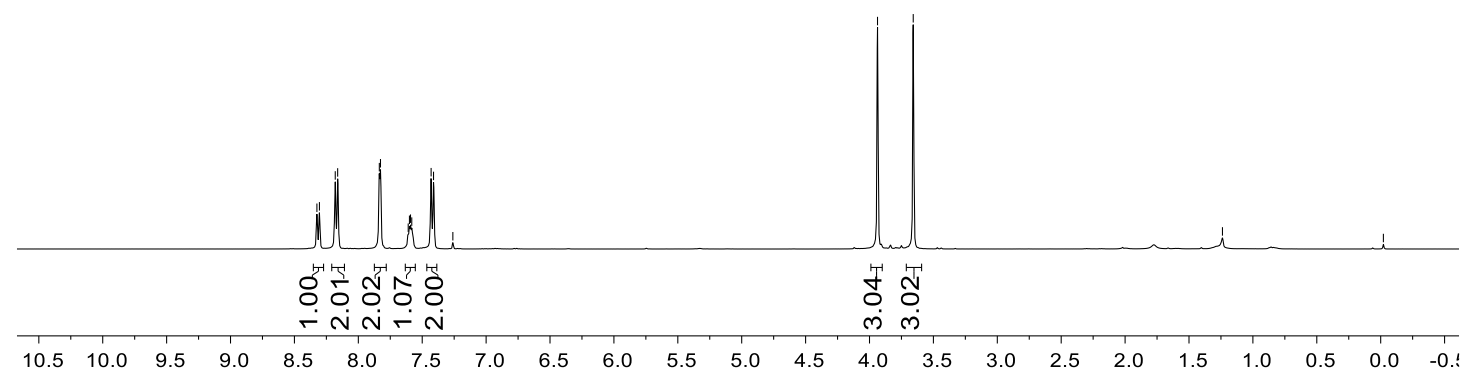

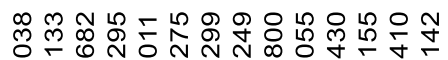

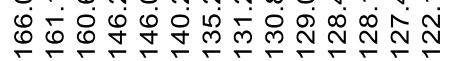

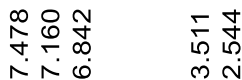

र요 政

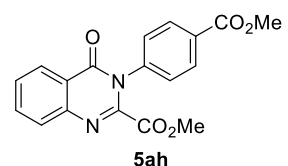

${ }^{13} \mathrm{C}, \mathrm{CDCl}_{3}, 100 \mathrm{MHz}$

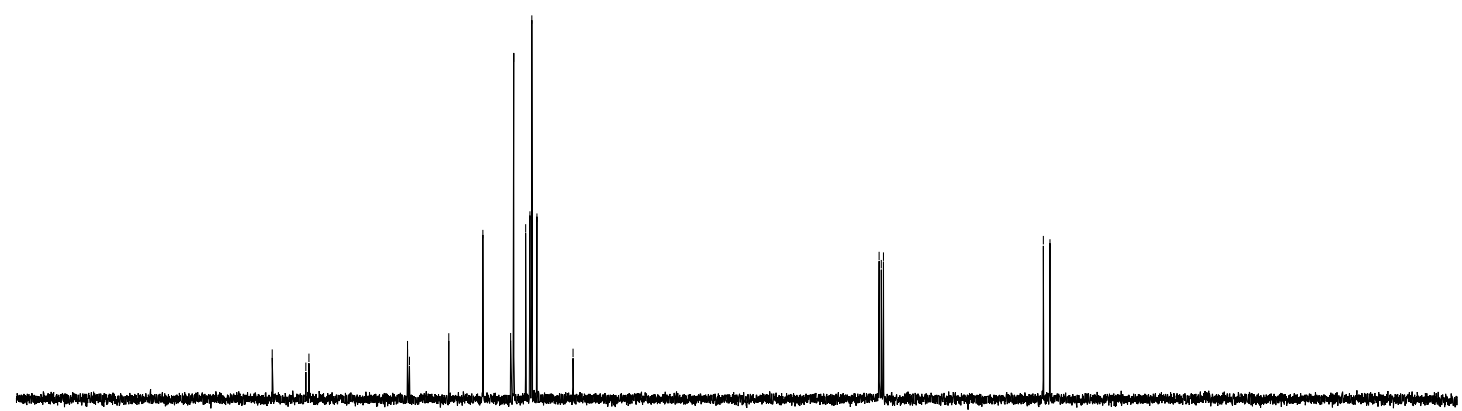

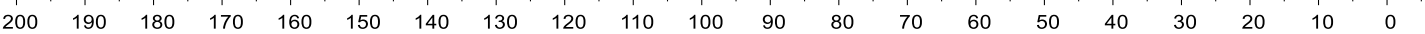




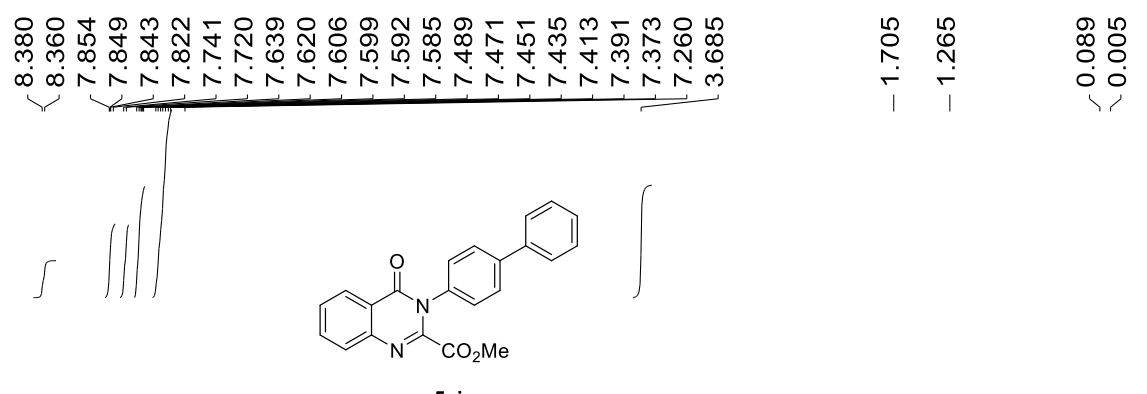

${ }^{1} \mathrm{H}, \mathrm{CDCl}_{3}, 400 \mathrm{MHz}$

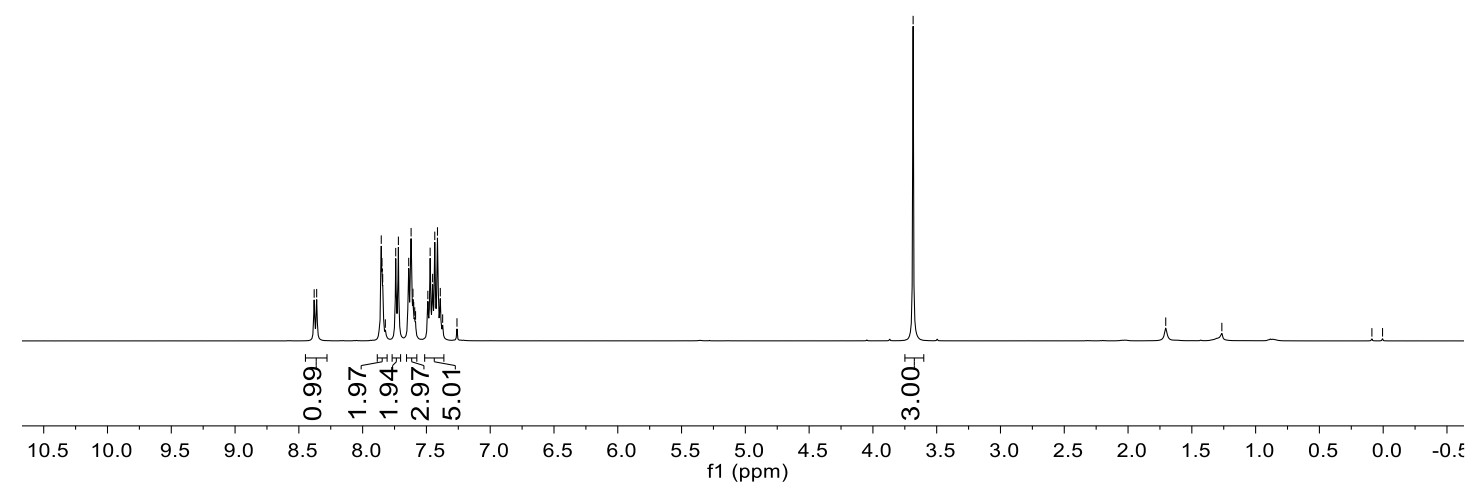

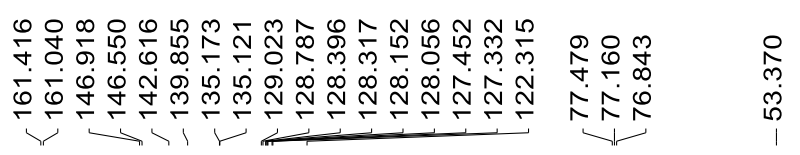

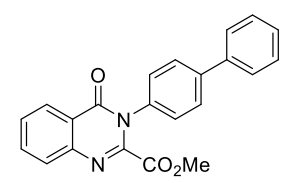

5 ai

${ }^{13} \mathrm{C}, \mathrm{CDCl}_{3}, 100 \mathrm{MHz}$

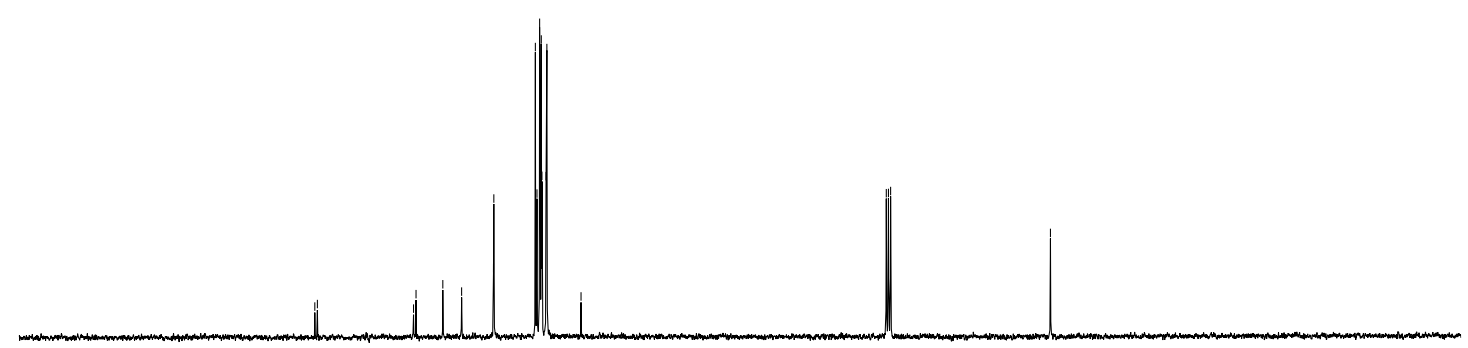

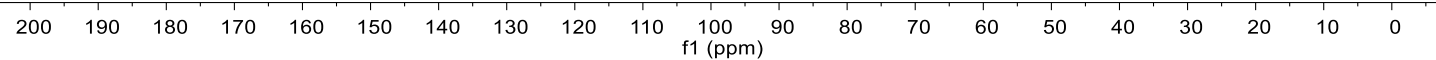



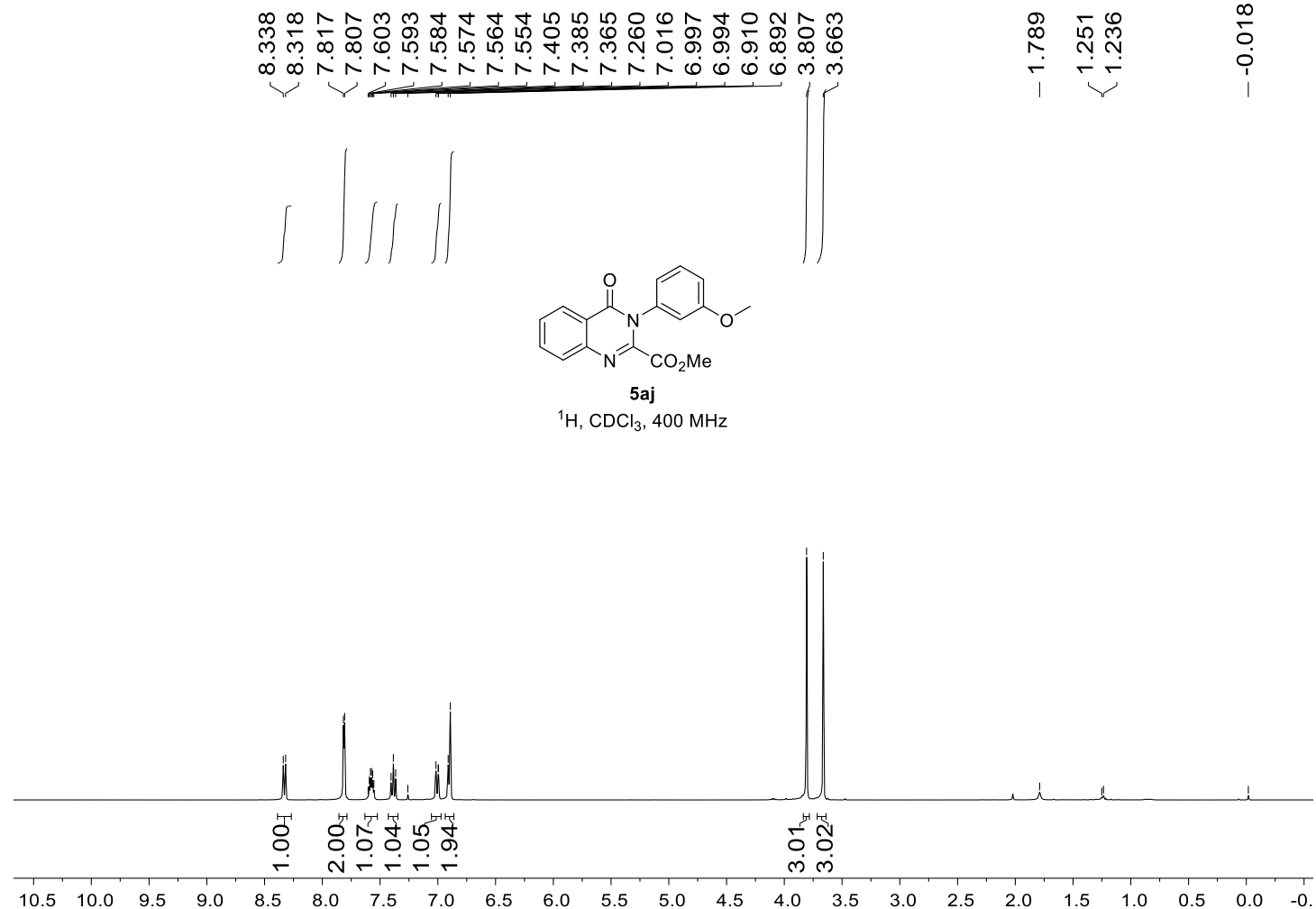

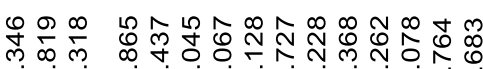

它家完

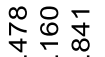

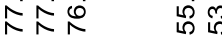

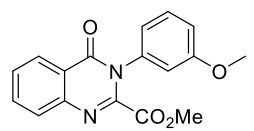

5 aj

${ }^{13} \mathrm{C}, \mathrm{CDCl}_{3}, 100 \mathrm{MHz}$

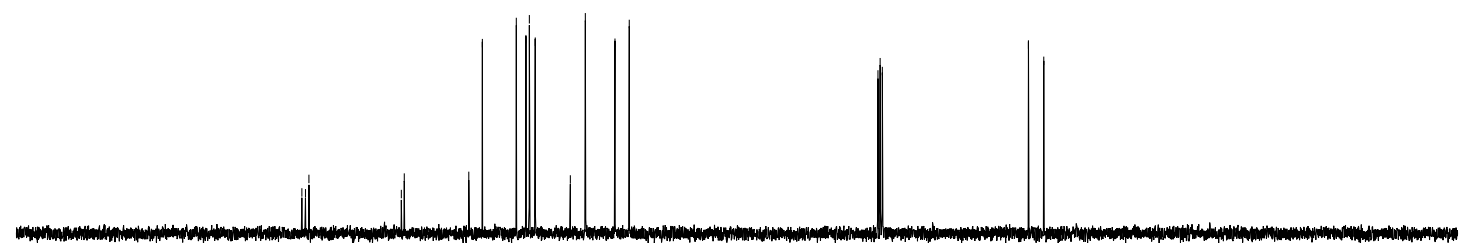

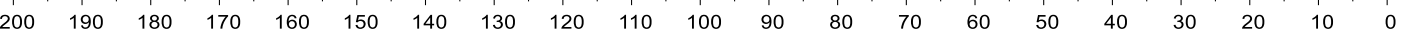




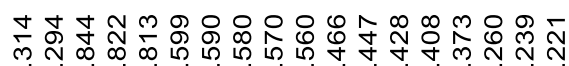

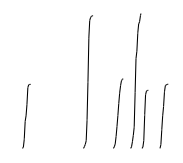

$$
\begin{aligned}
& 5 \\
& 0 \\
& 0 \\
& 1
\end{aligned}
$$

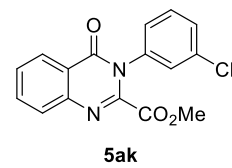

${ }^{1} \mathrm{H}, \mathrm{CDCl}_{3}, 400 \mathrm{MHz}$

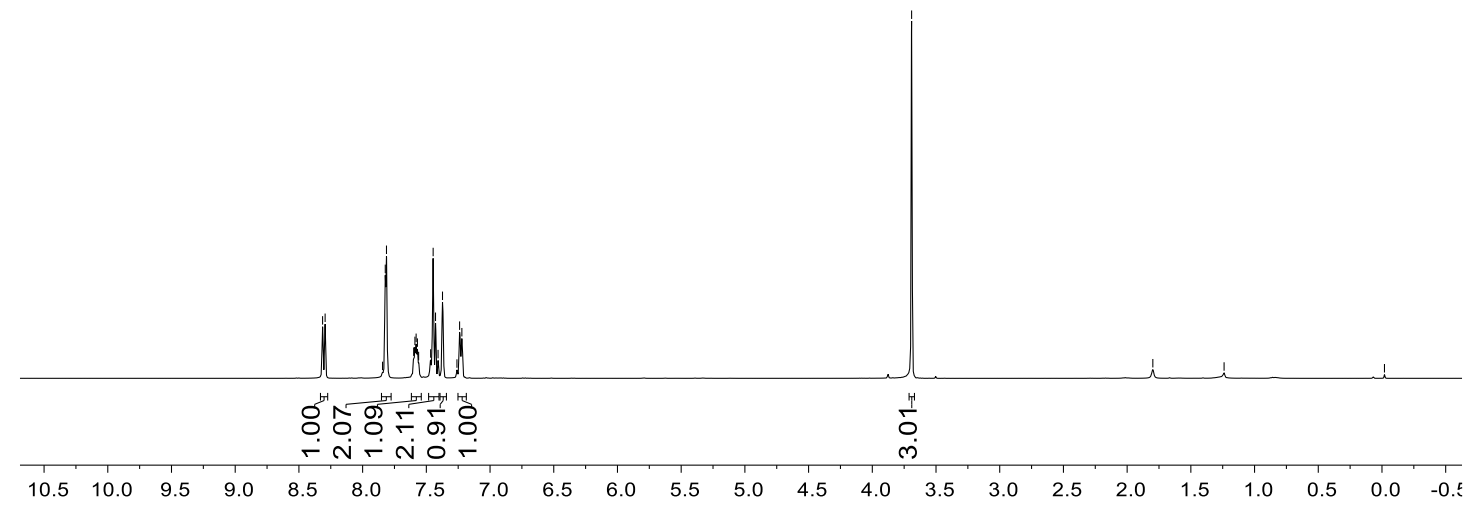

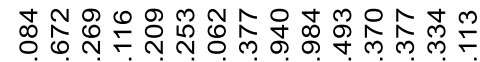

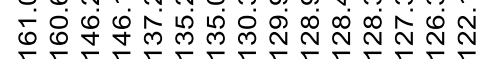

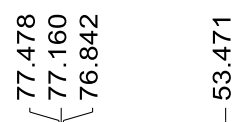
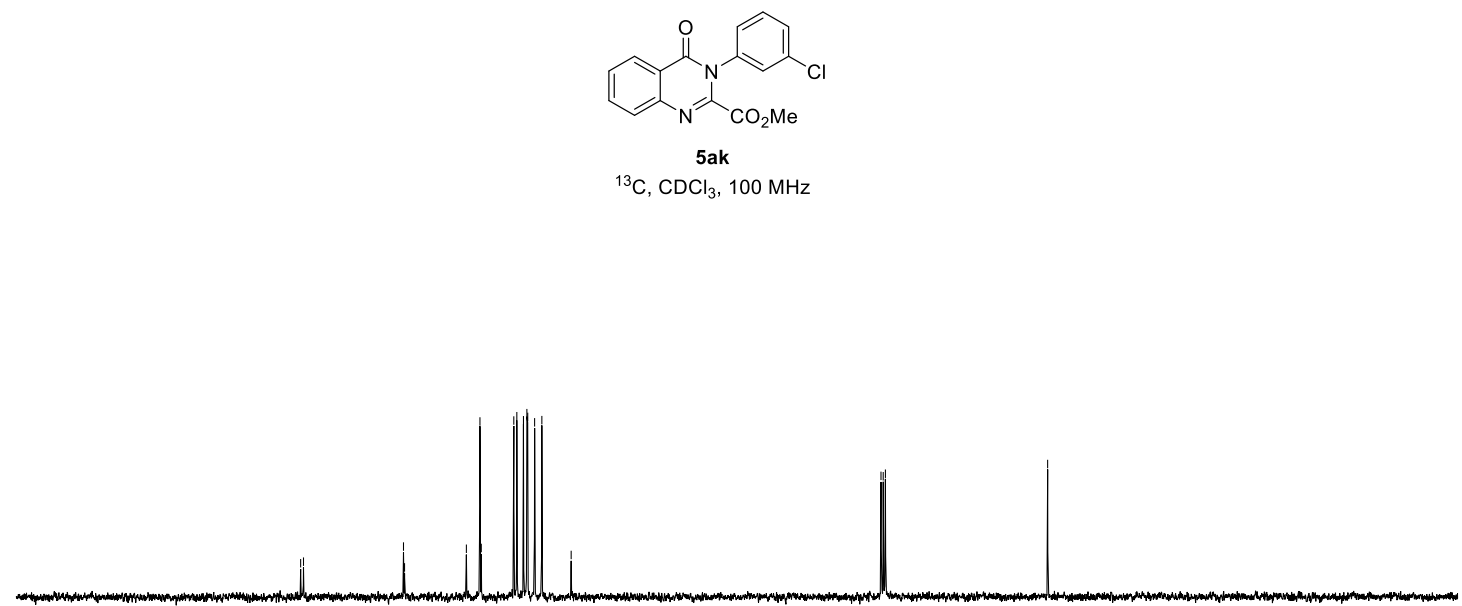

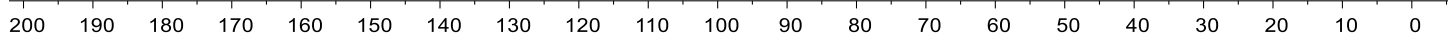



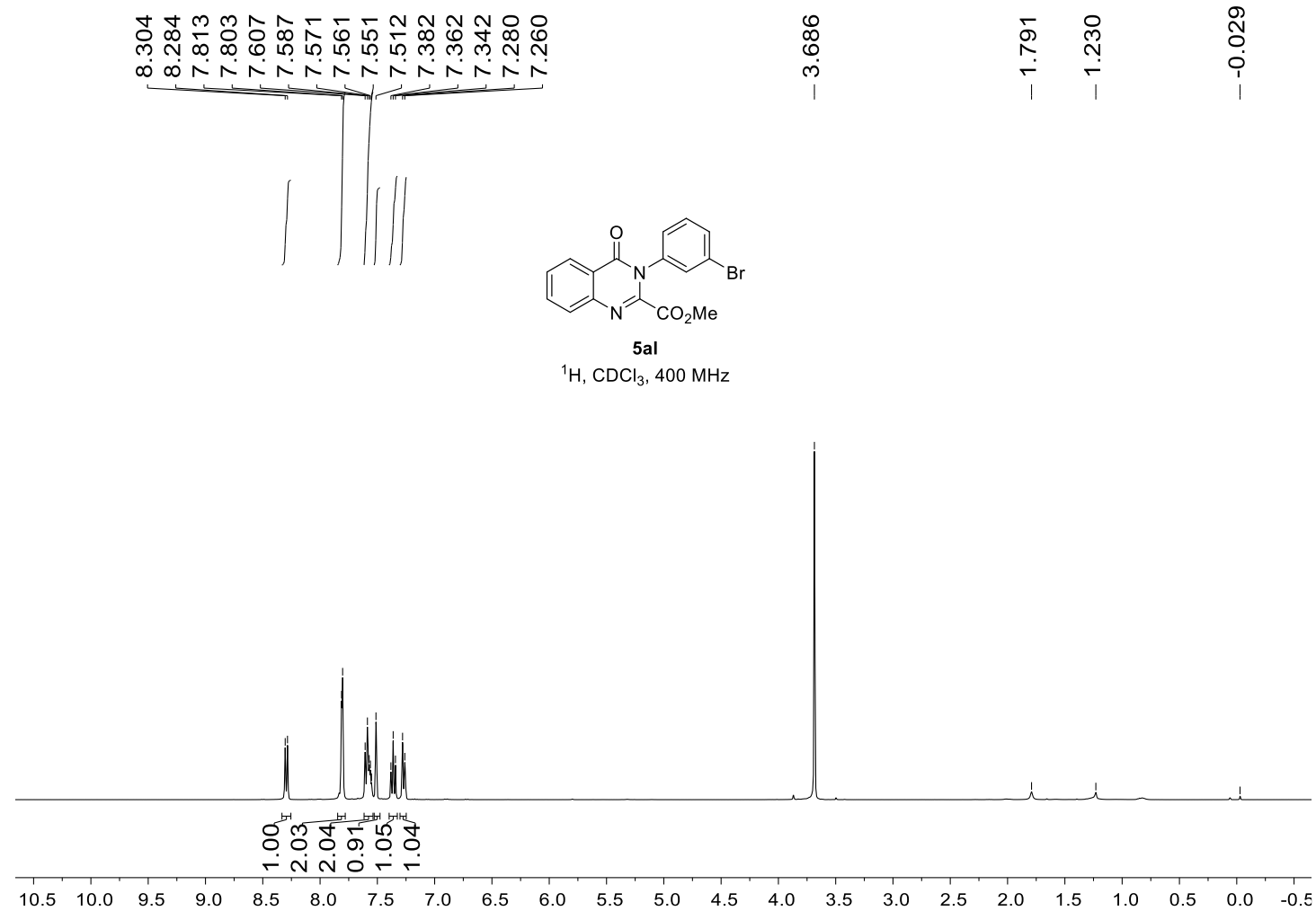

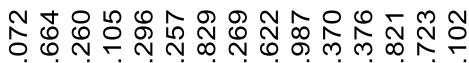

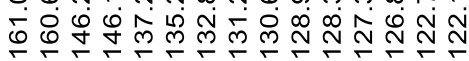

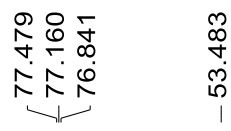

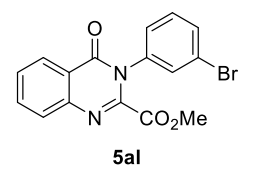

${ }^{13} \mathrm{C}, \mathrm{CDCl}_{3}, 100 \mathrm{MHz}$

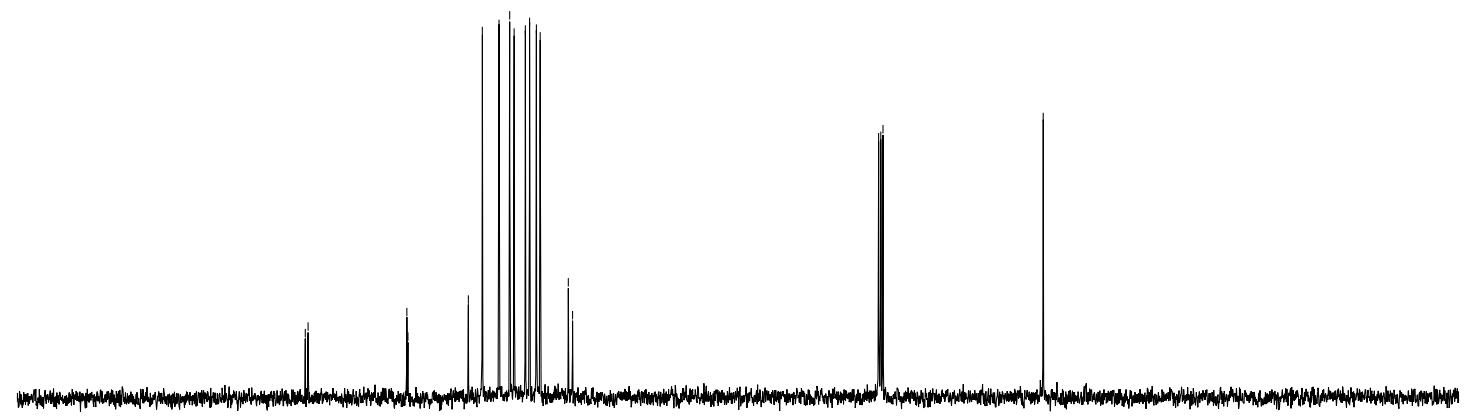

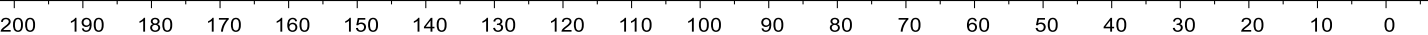



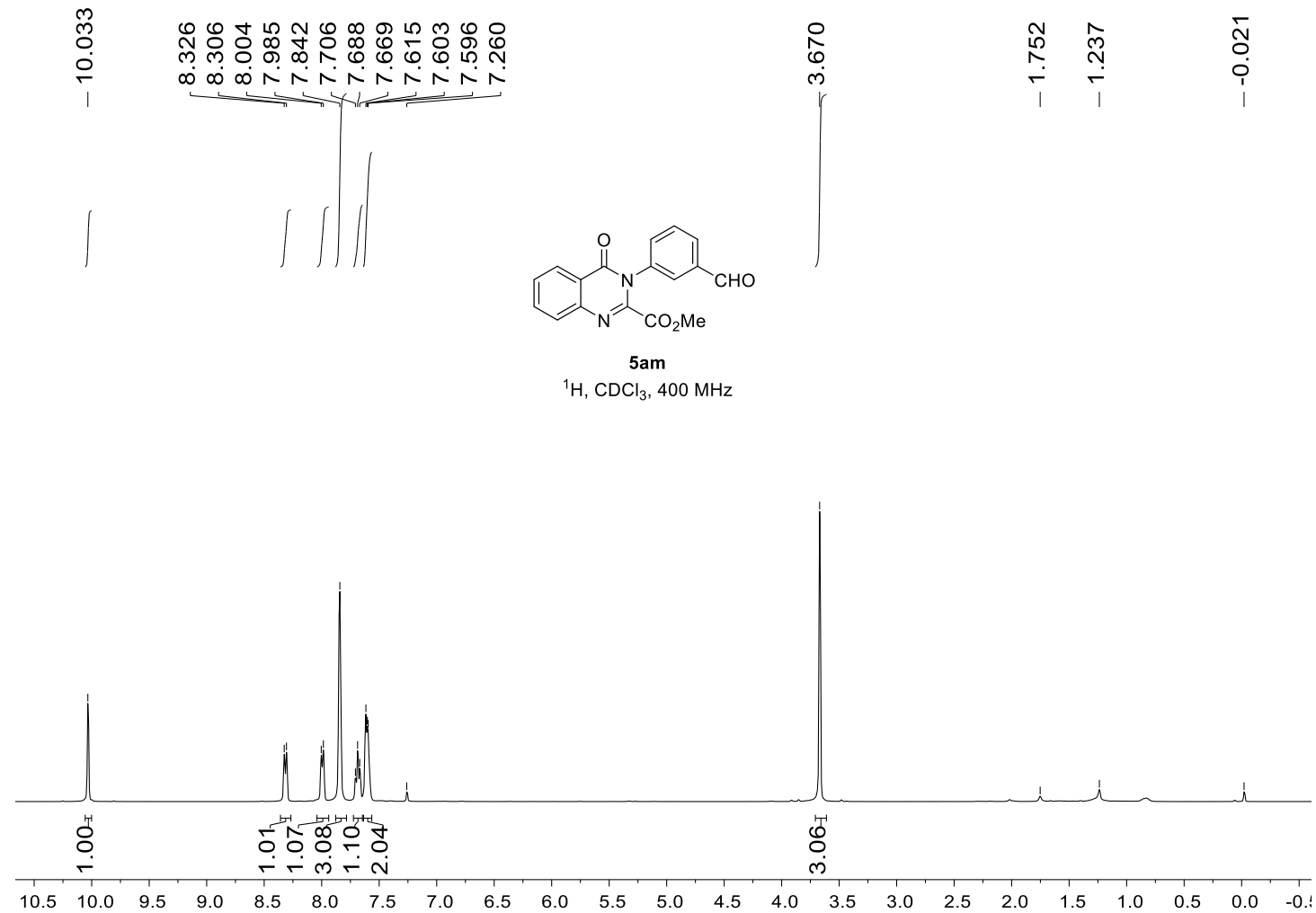

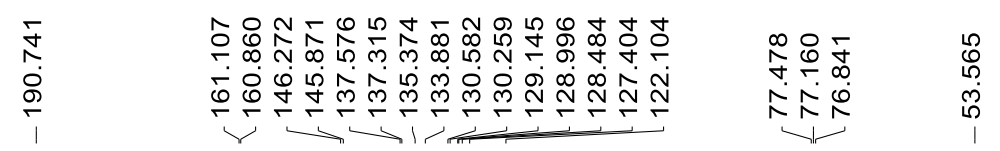
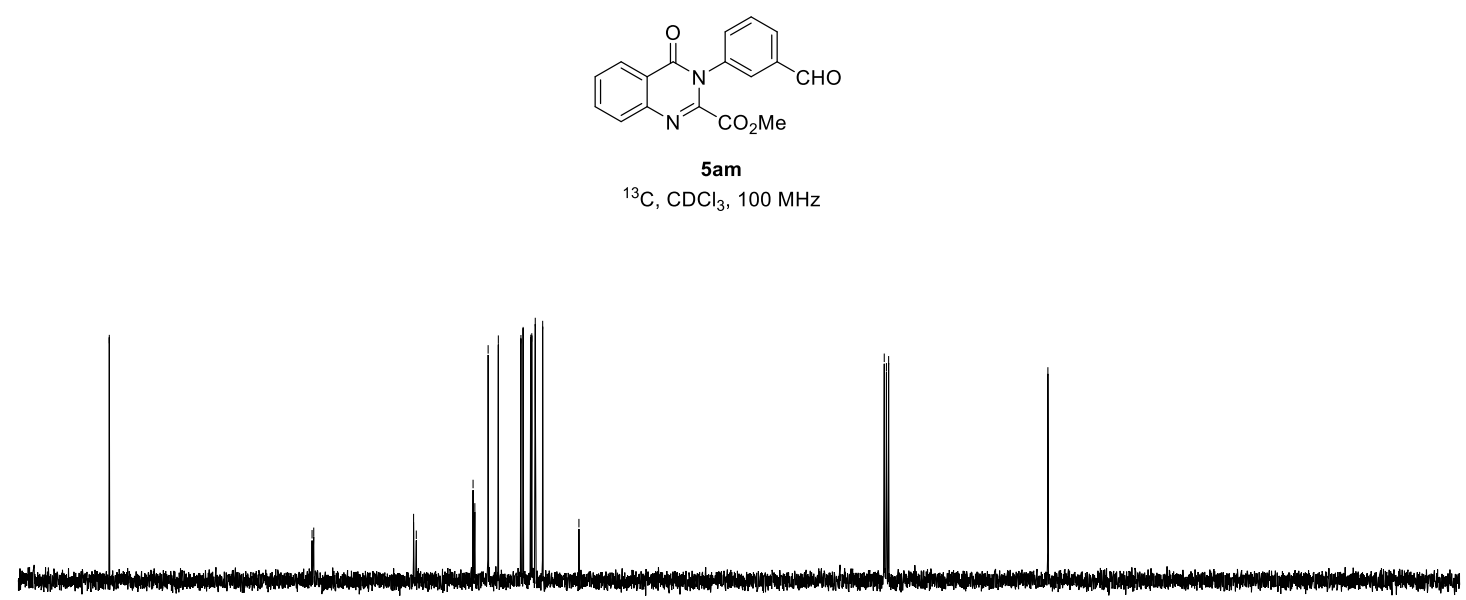

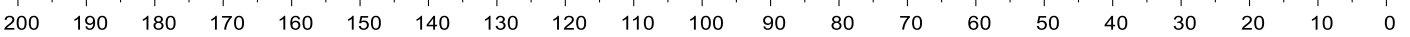




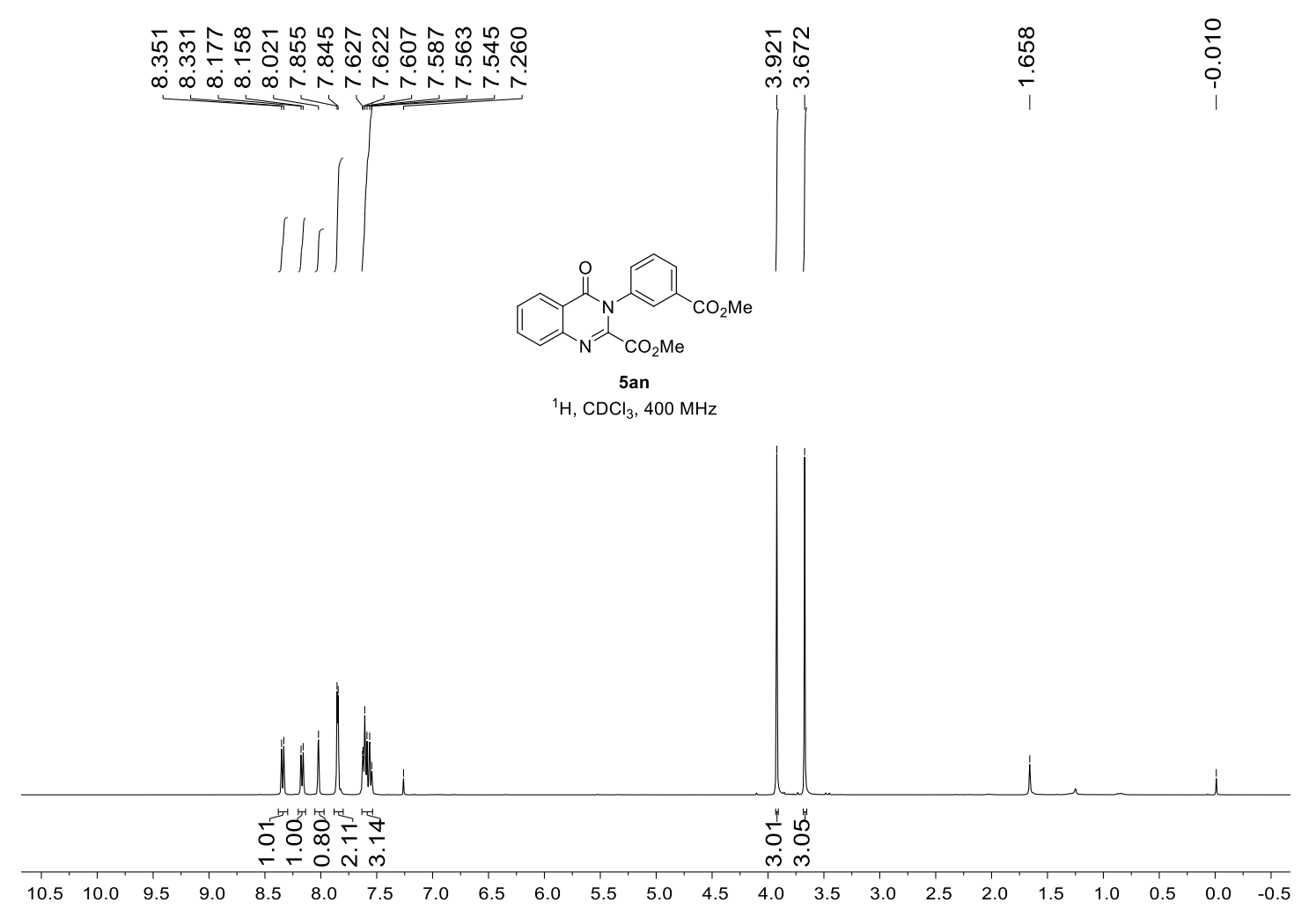

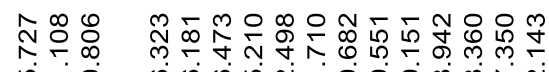

它家

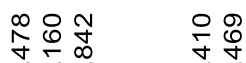

소

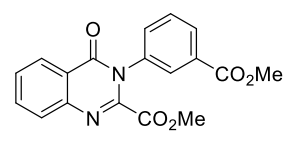

${ }^{13} \mathrm{C}, \mathrm{CDCl}_{3}, 100 \mathrm{MHz}$

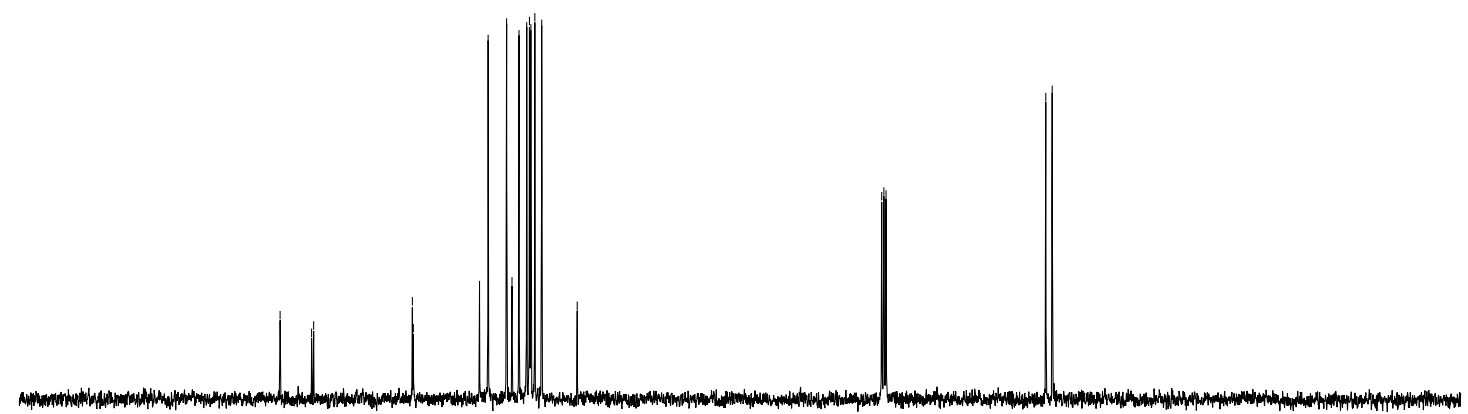

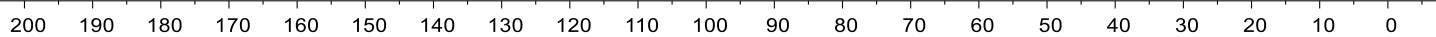




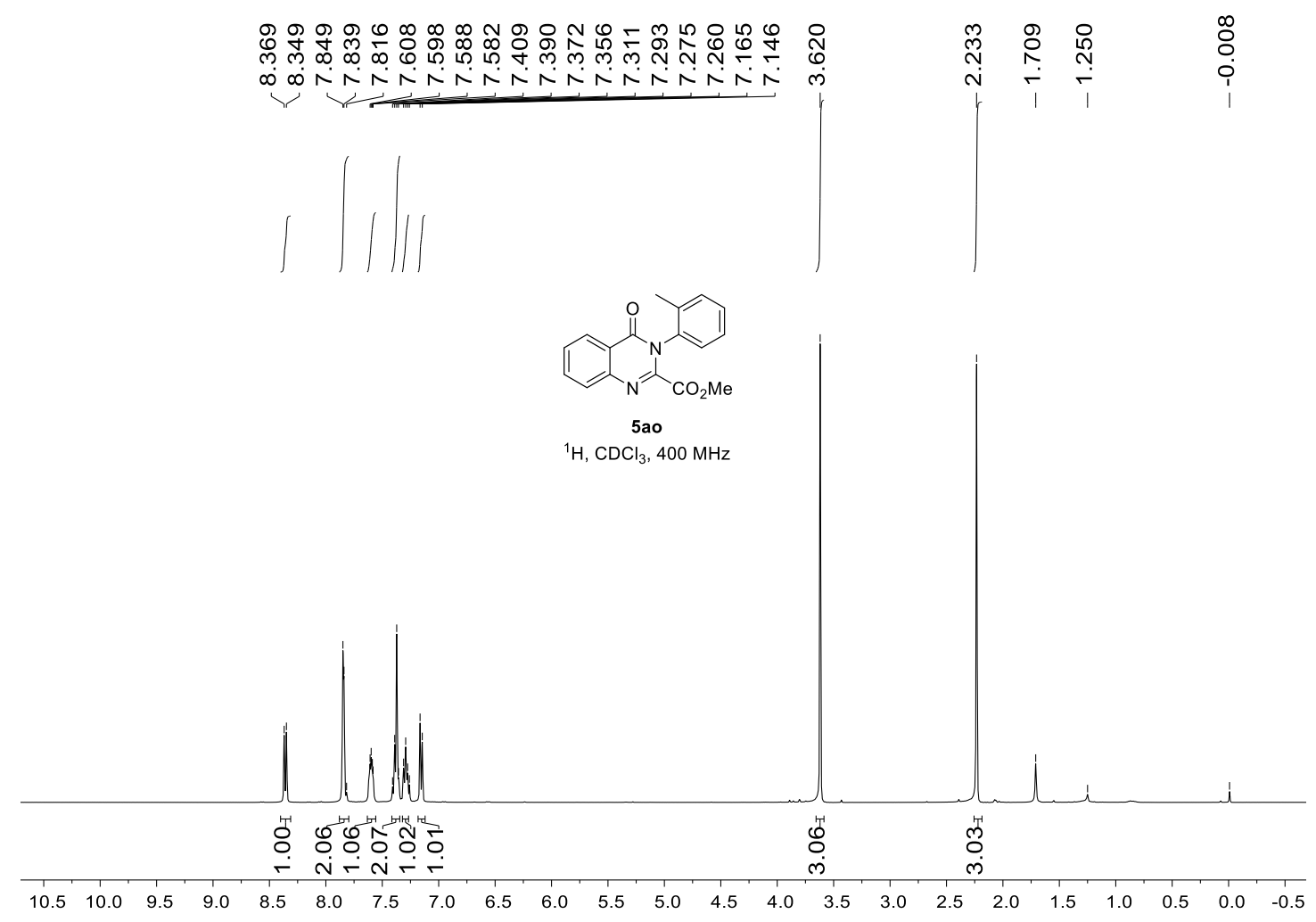

倠等

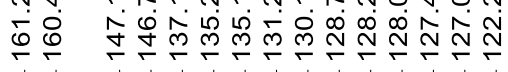

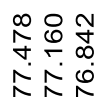

$\stackrel{\infty}{\infty}$

走完

$\infty$
0
$\infty$

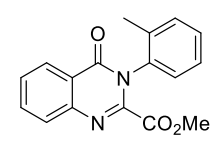

5 ao

${ }^{13} \mathrm{C}, \mathrm{CDCl}_{3}, 100 \mathrm{MHz}$

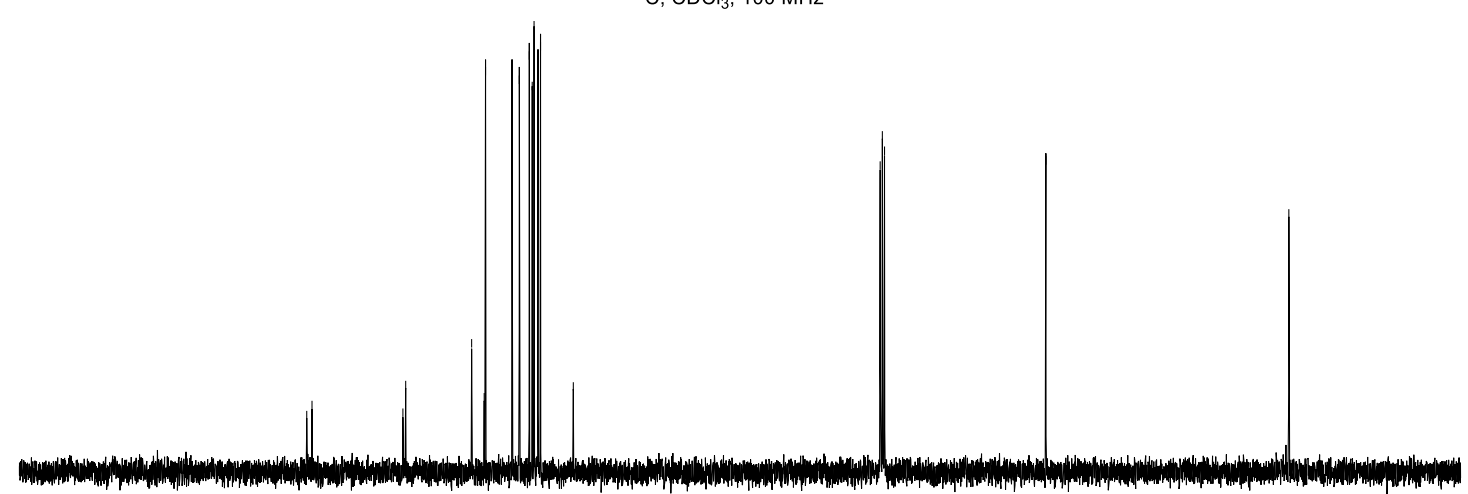

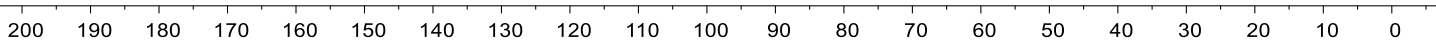




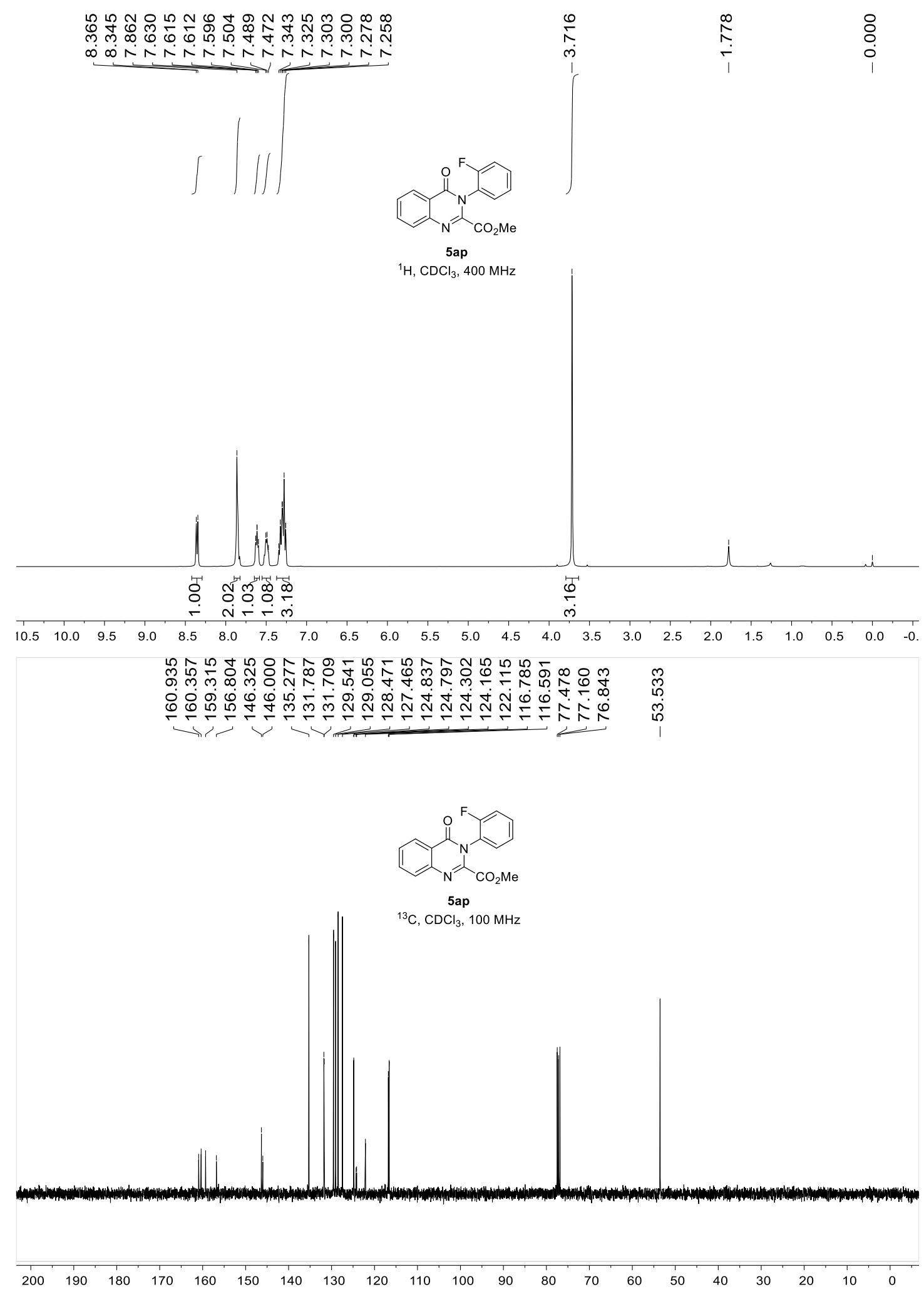




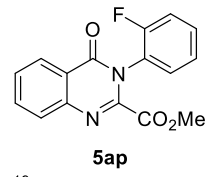

${ }^{19} \mathrm{~F}, \mathrm{CDCl}_{3}, 376 \mathrm{MHz}$

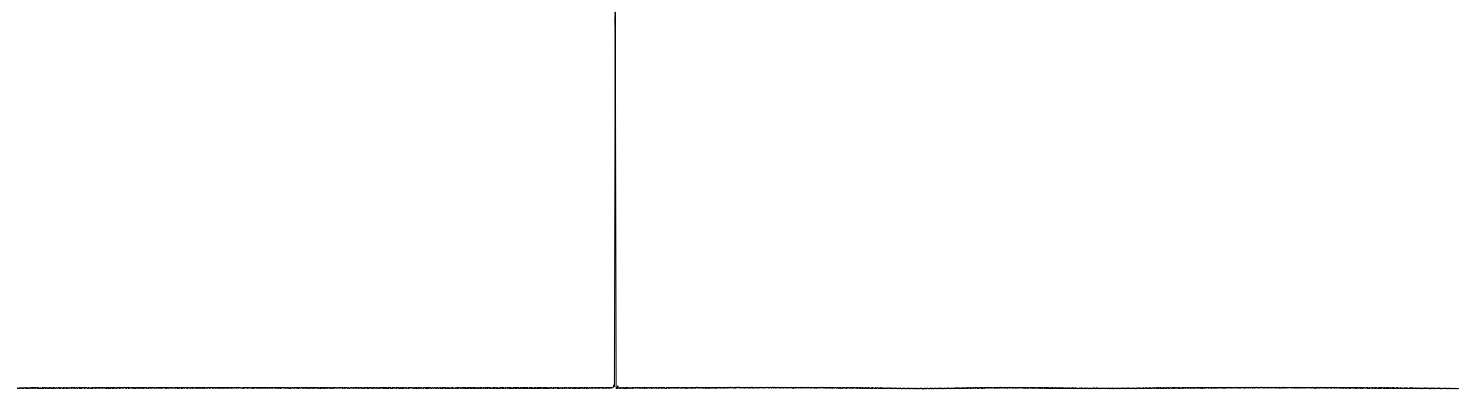

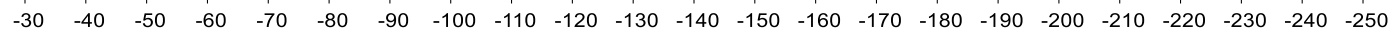




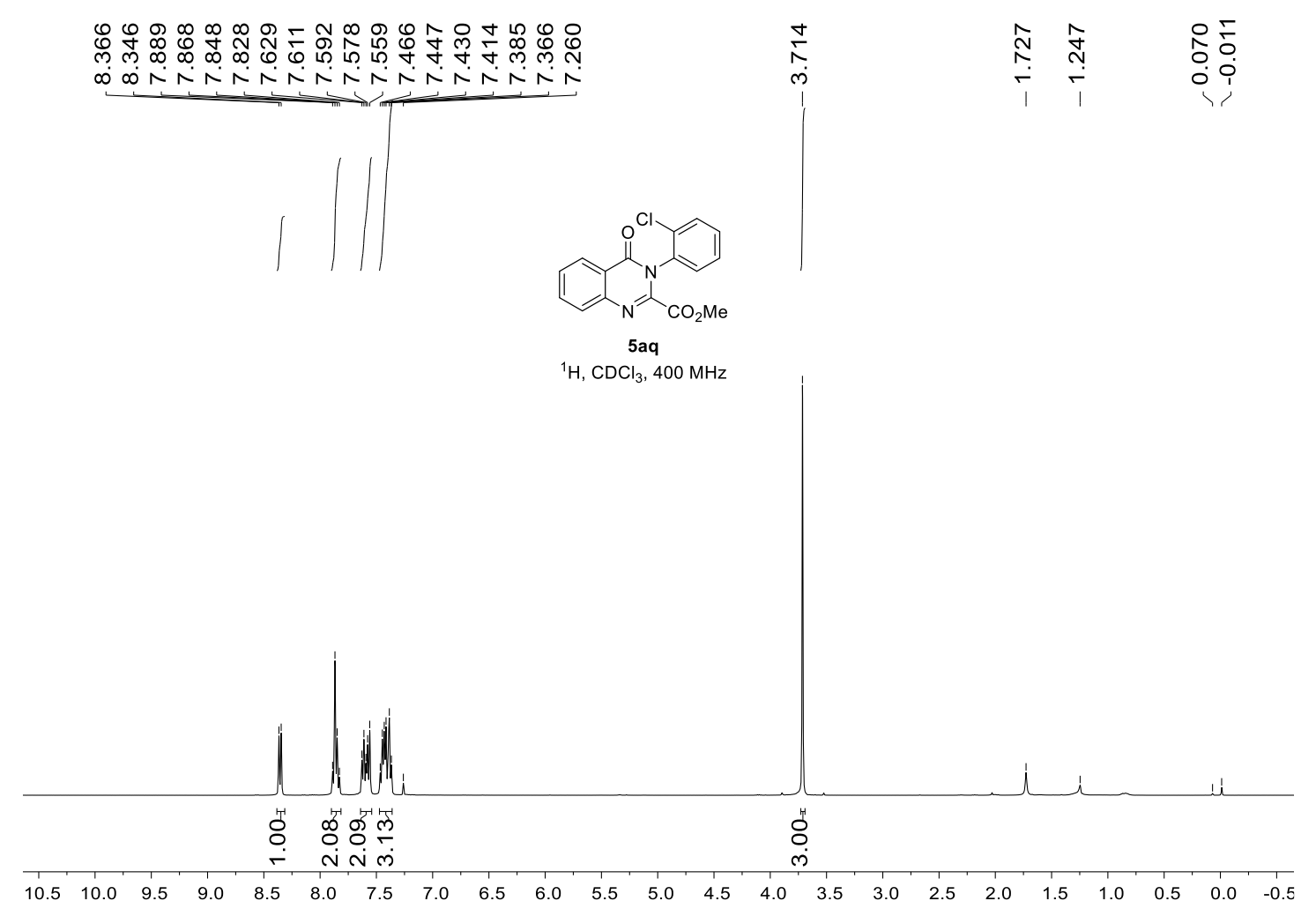

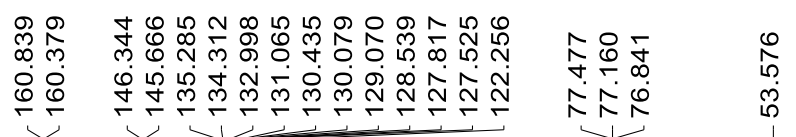

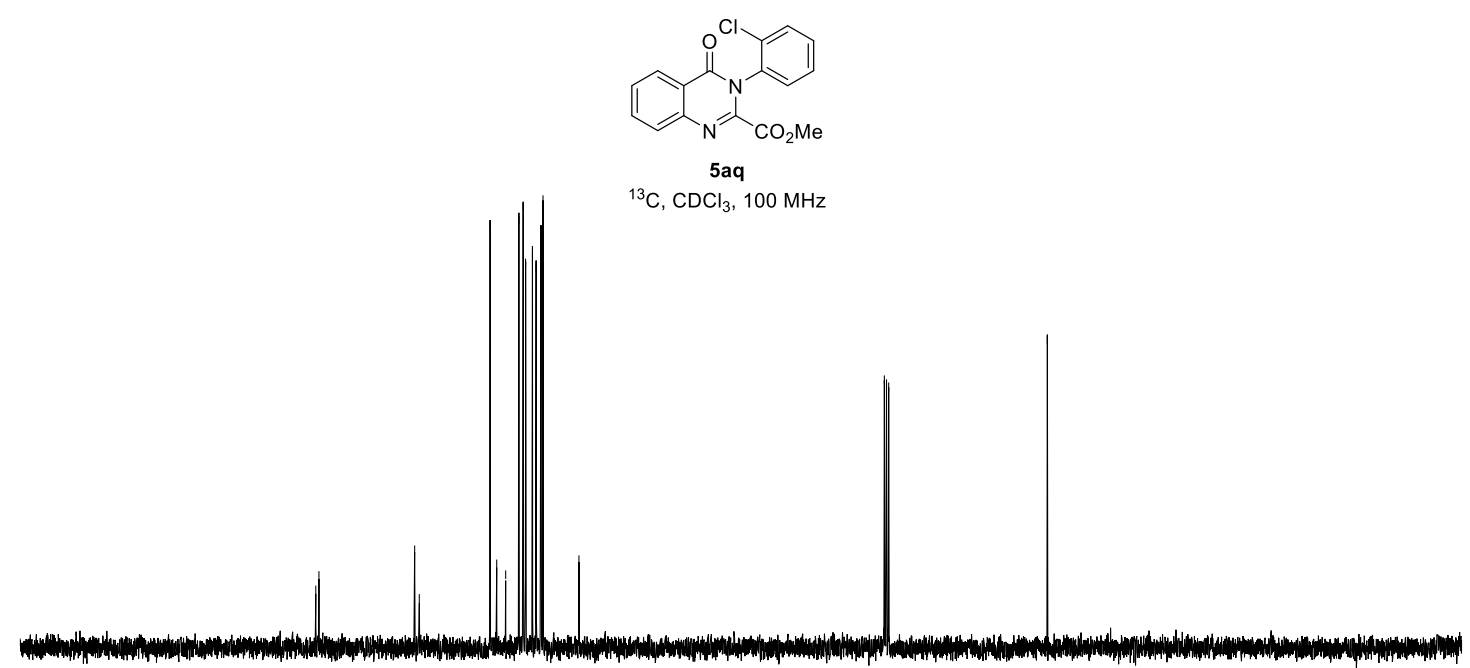

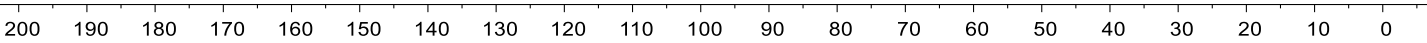




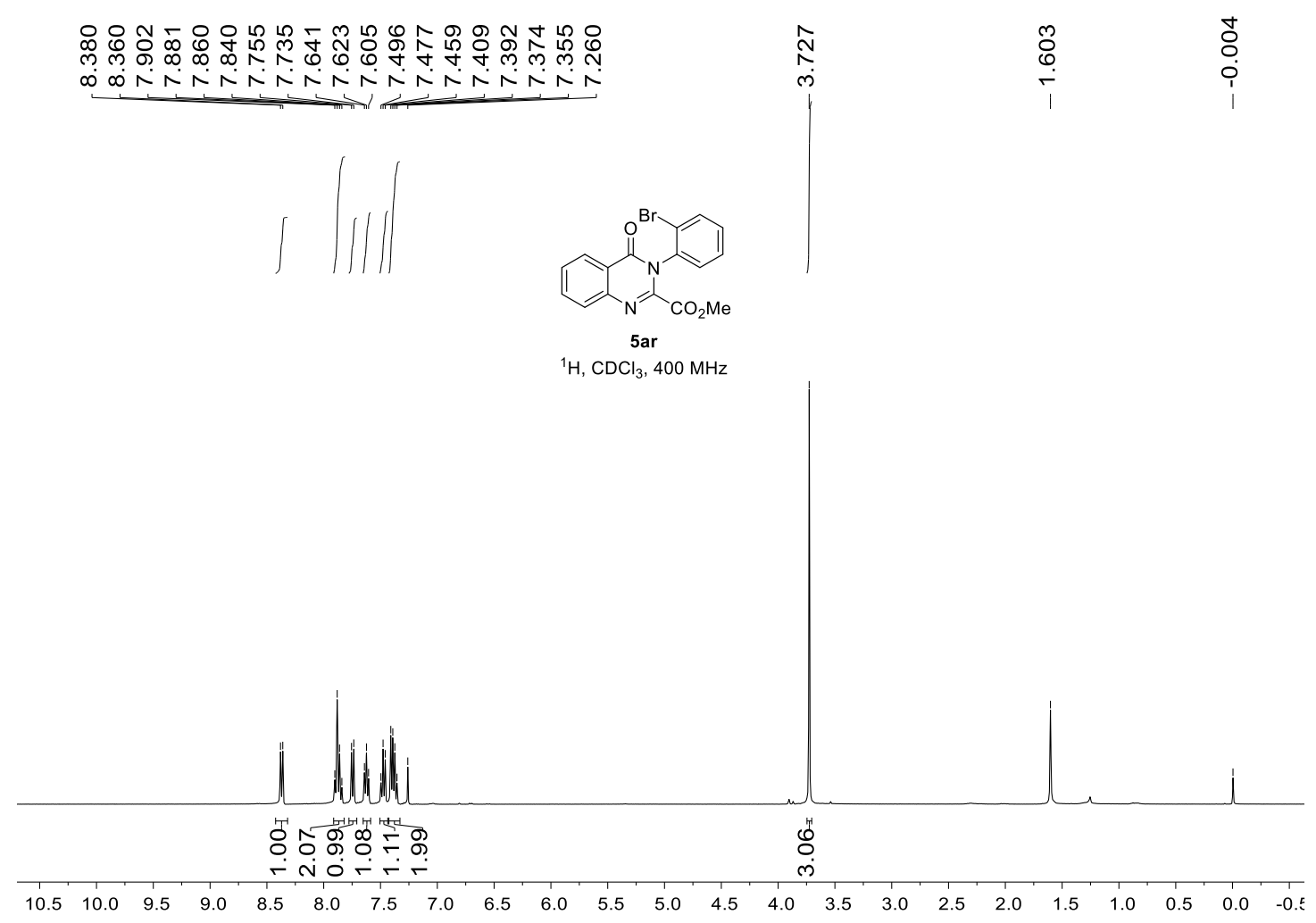

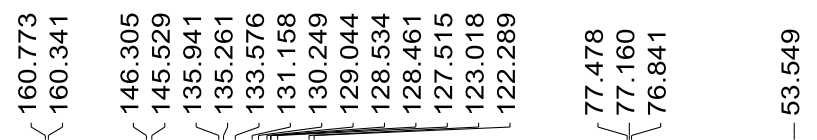

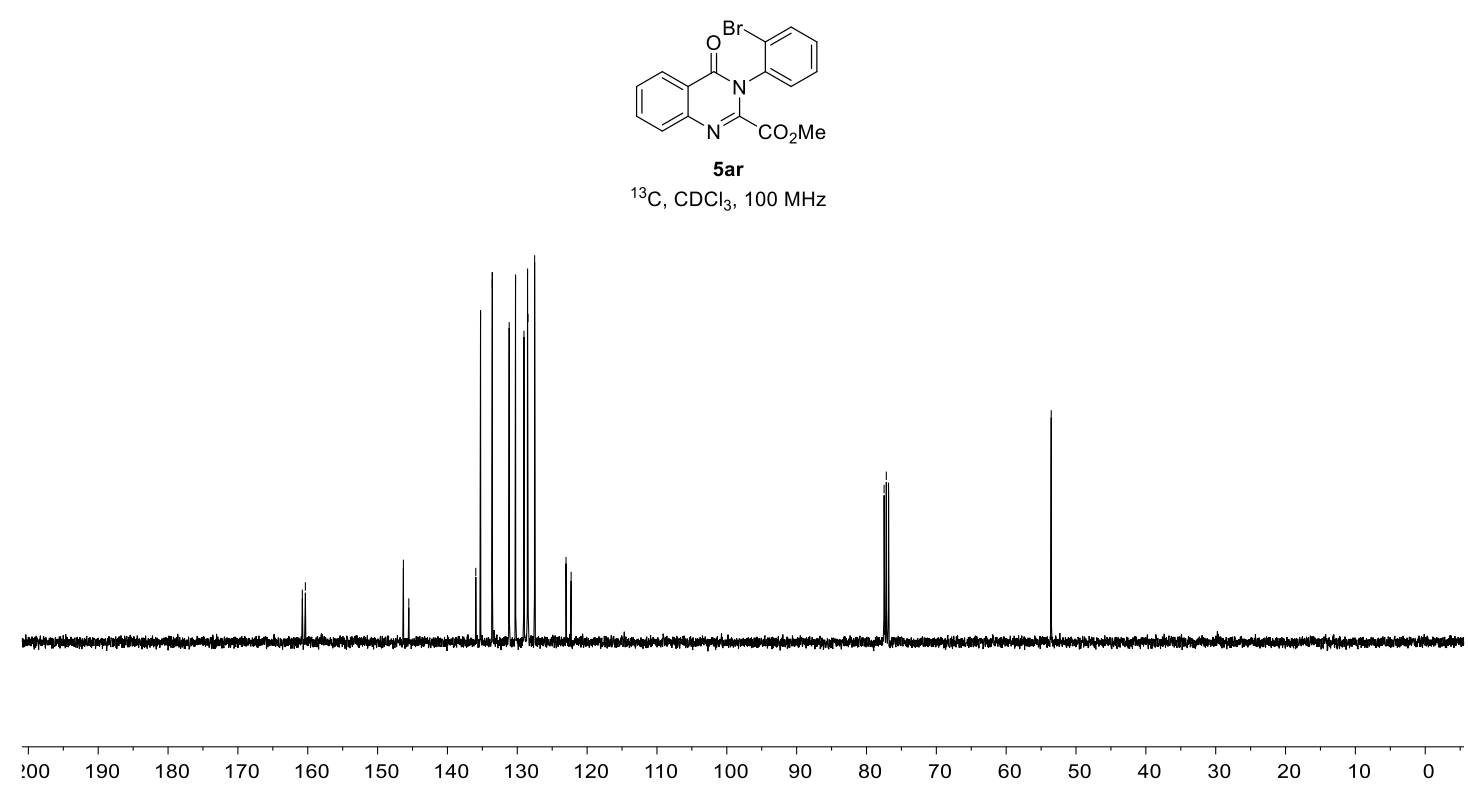




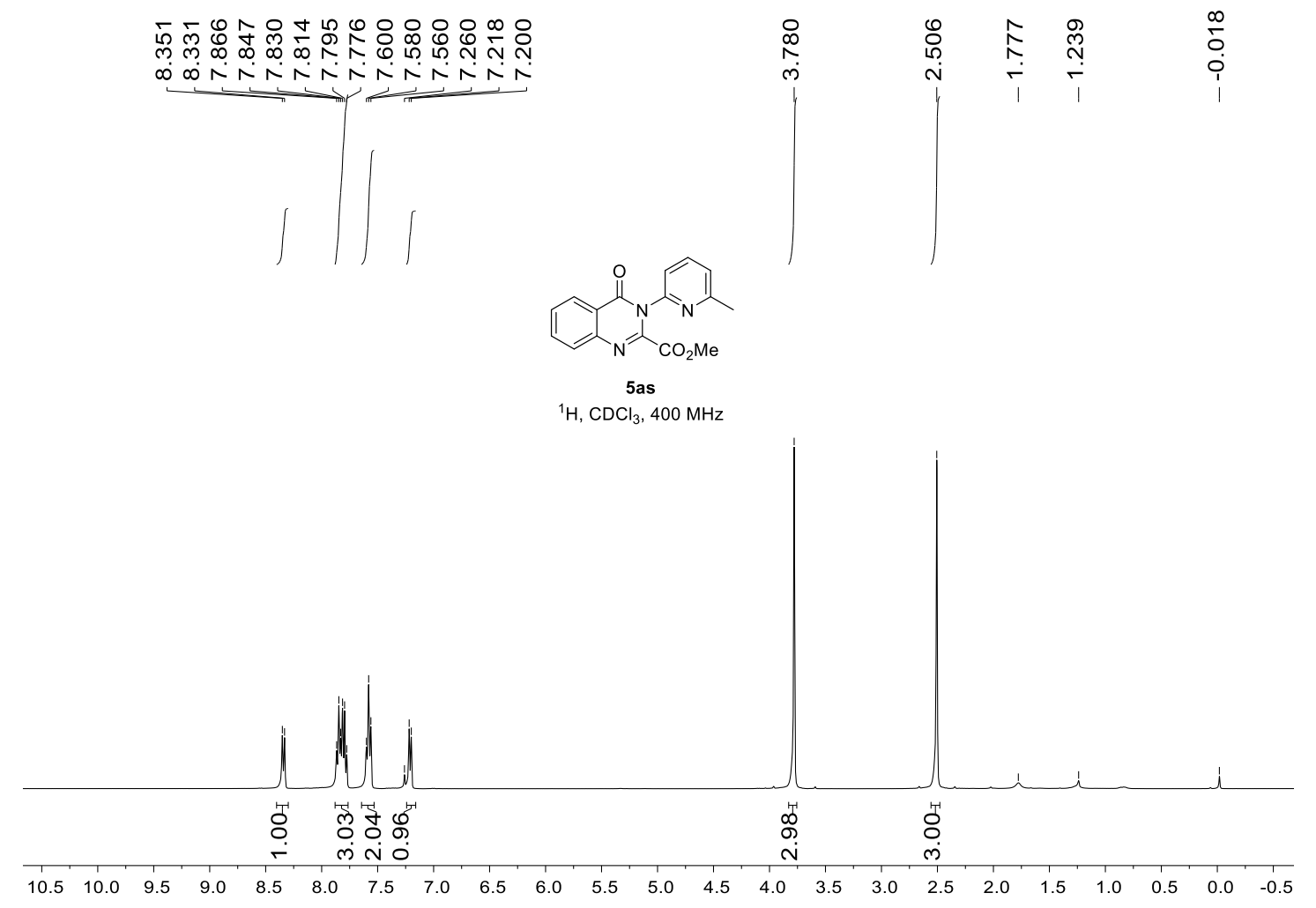

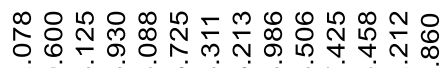

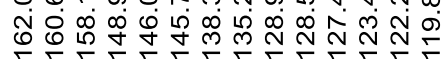

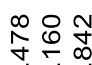

रํํㅇ

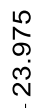

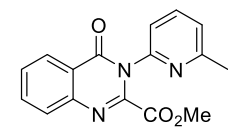

5 as

${ }^{13} \mathrm{C}, \mathrm{CDCl}_{3}, 100 \mathrm{MHz}$

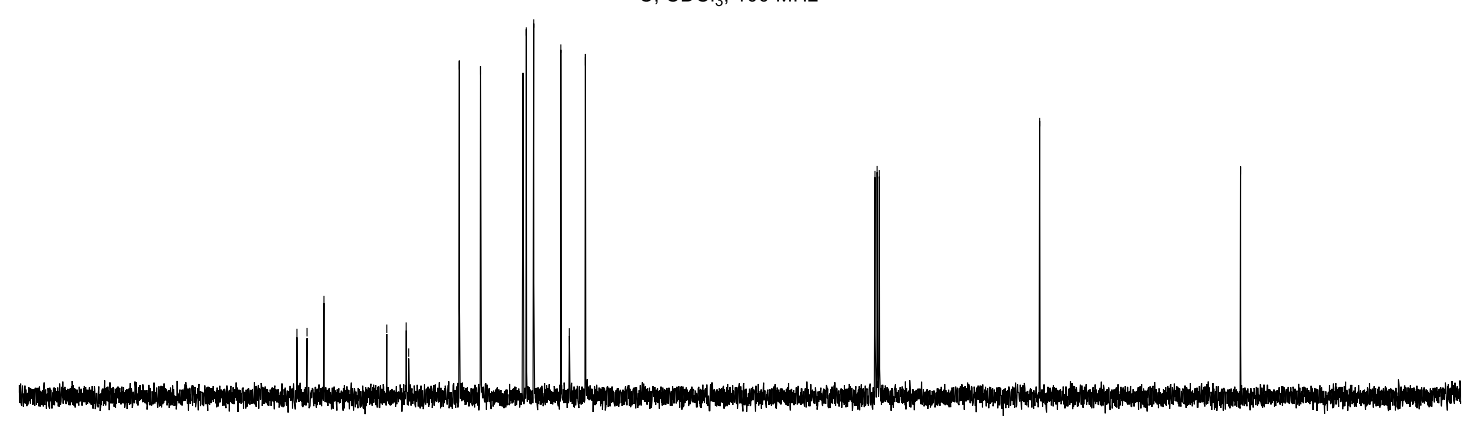

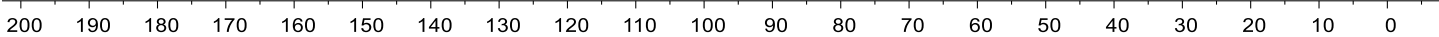




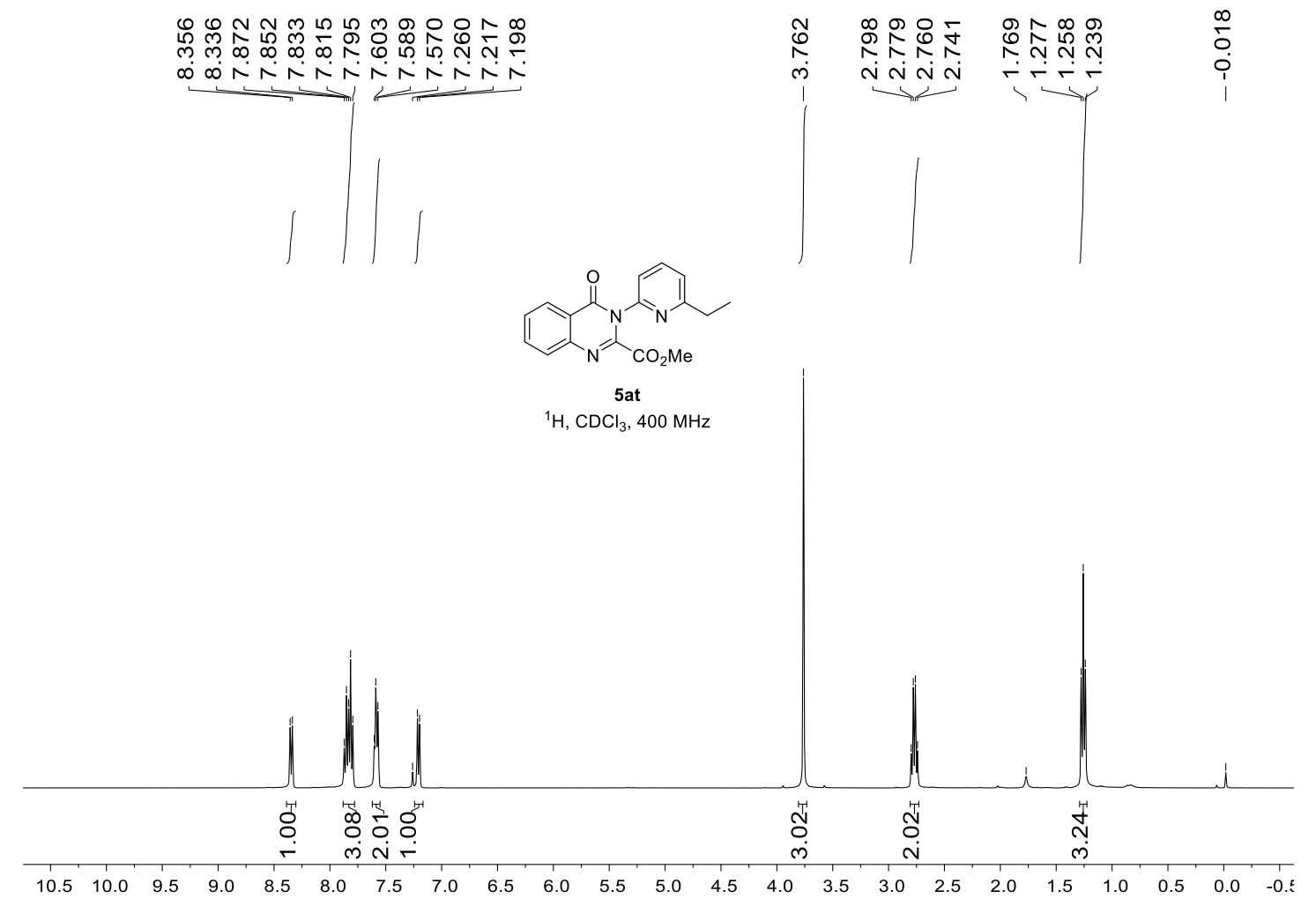

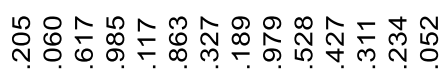

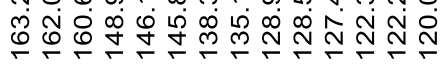

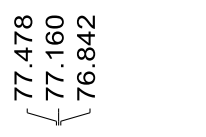

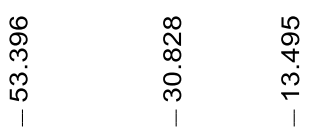

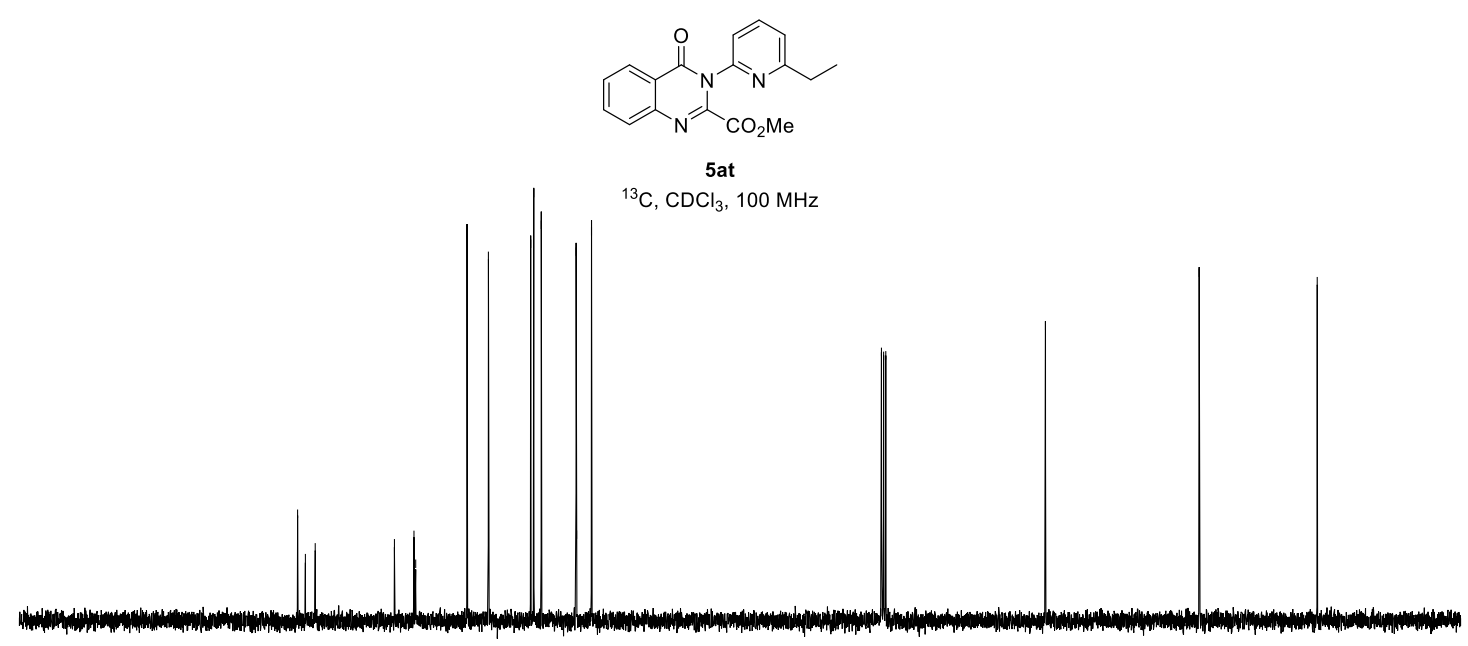

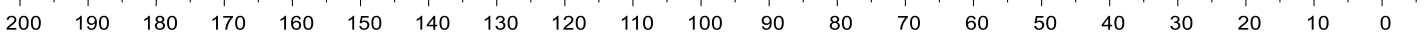




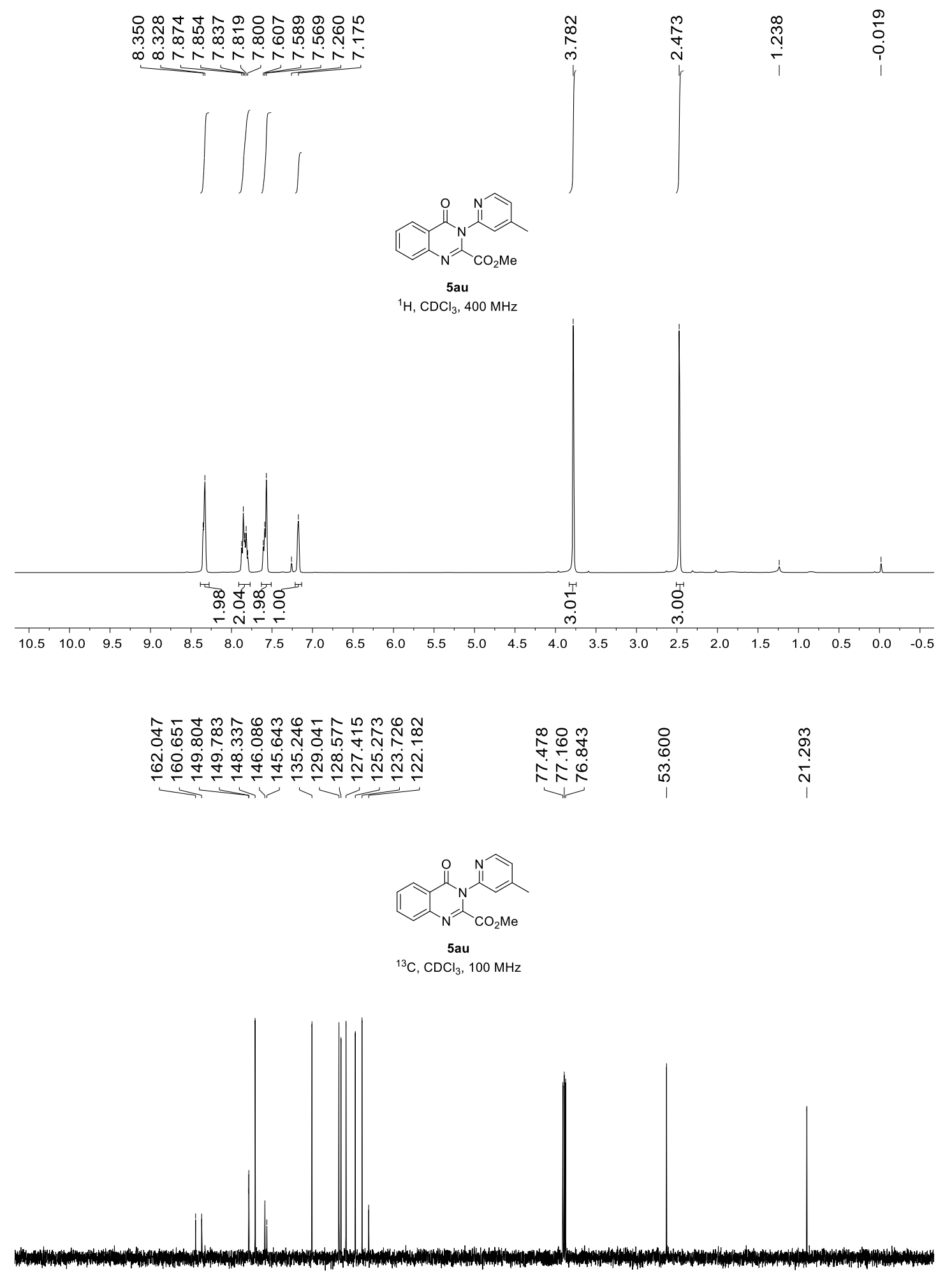

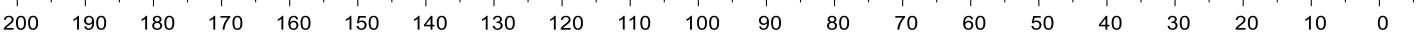




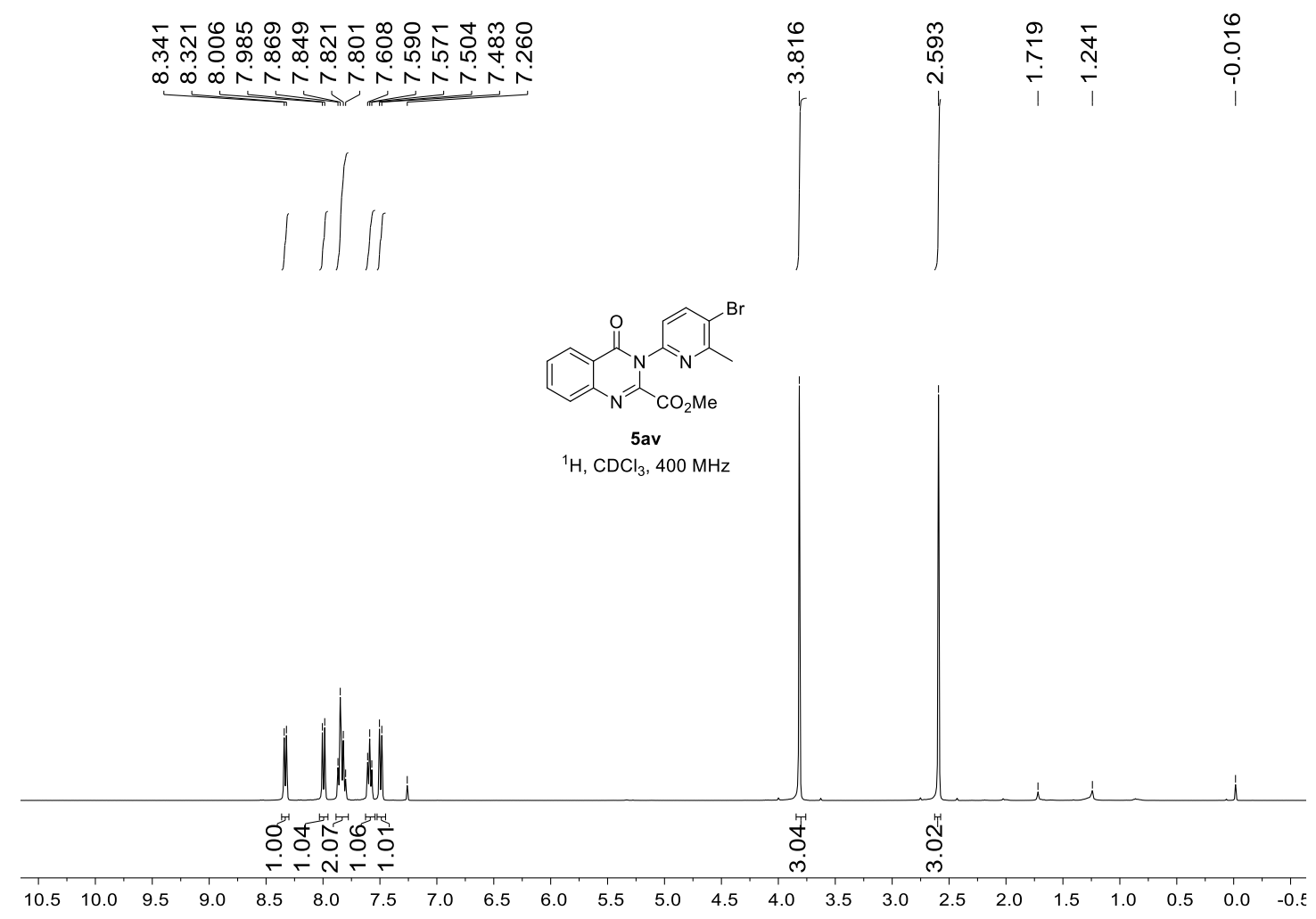

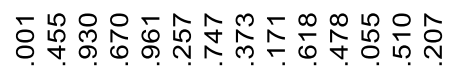

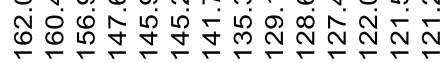

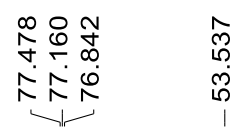

্ָণ

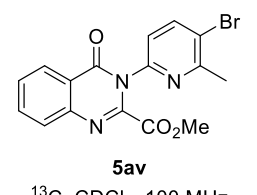

${ }^{13} \mathrm{C}, \mathrm{CDCl}_{3}, 100 \mathrm{MHz}$

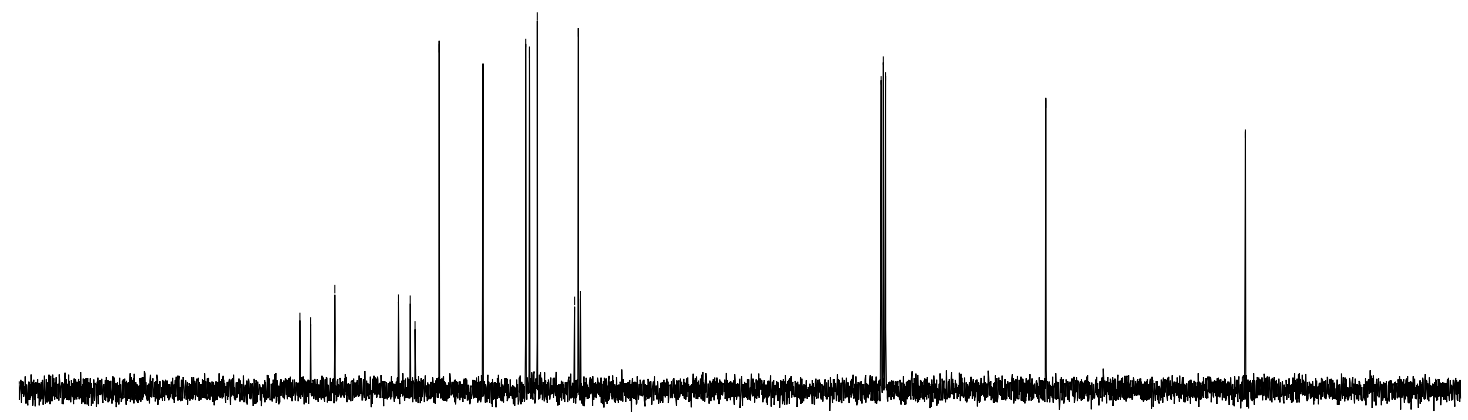

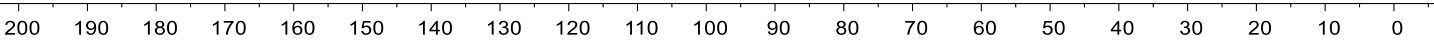



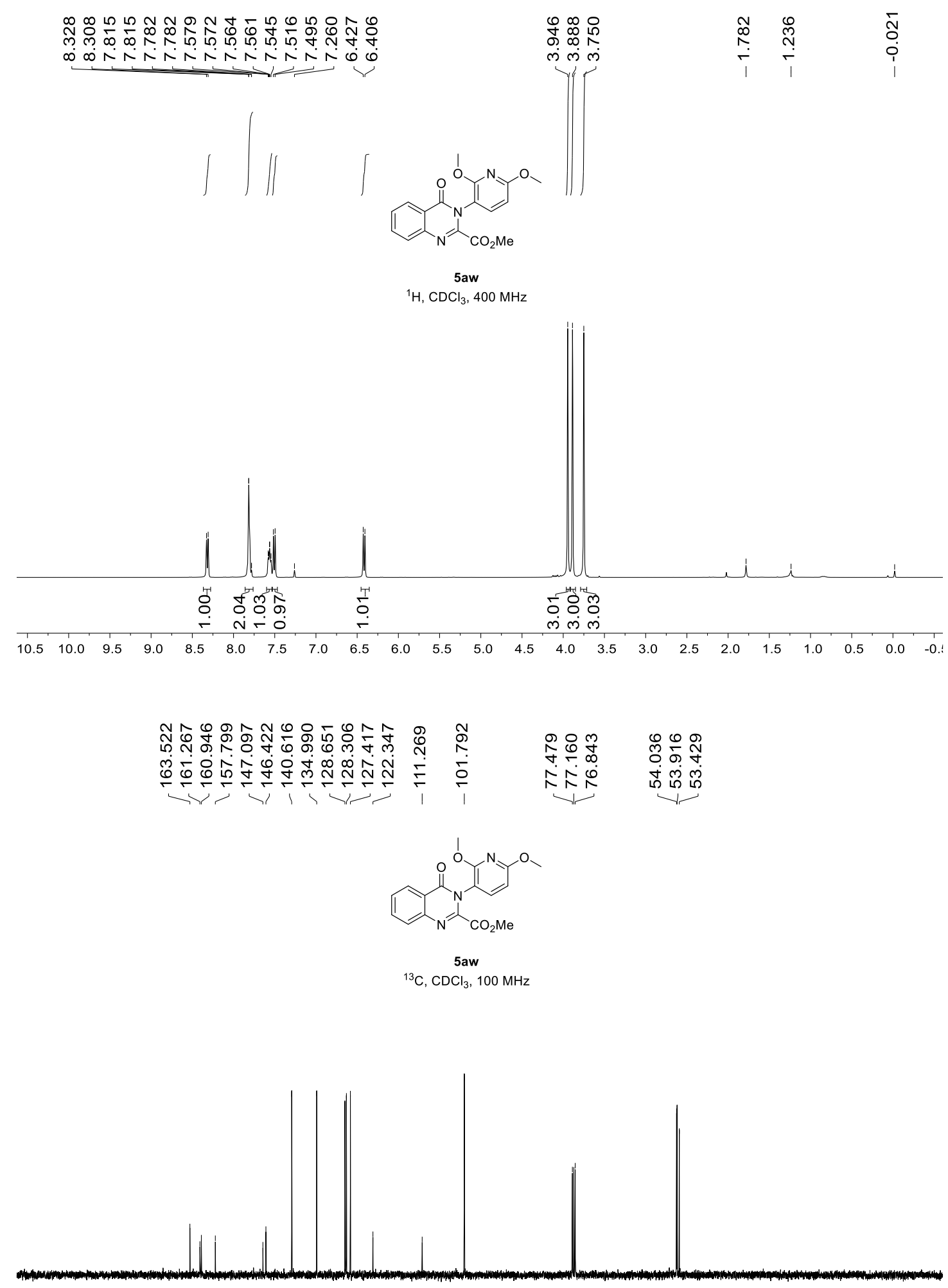

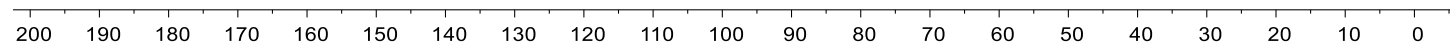


운운융 $\infty \infty_{\infty}^{\infty} \infty N \hat{N}$

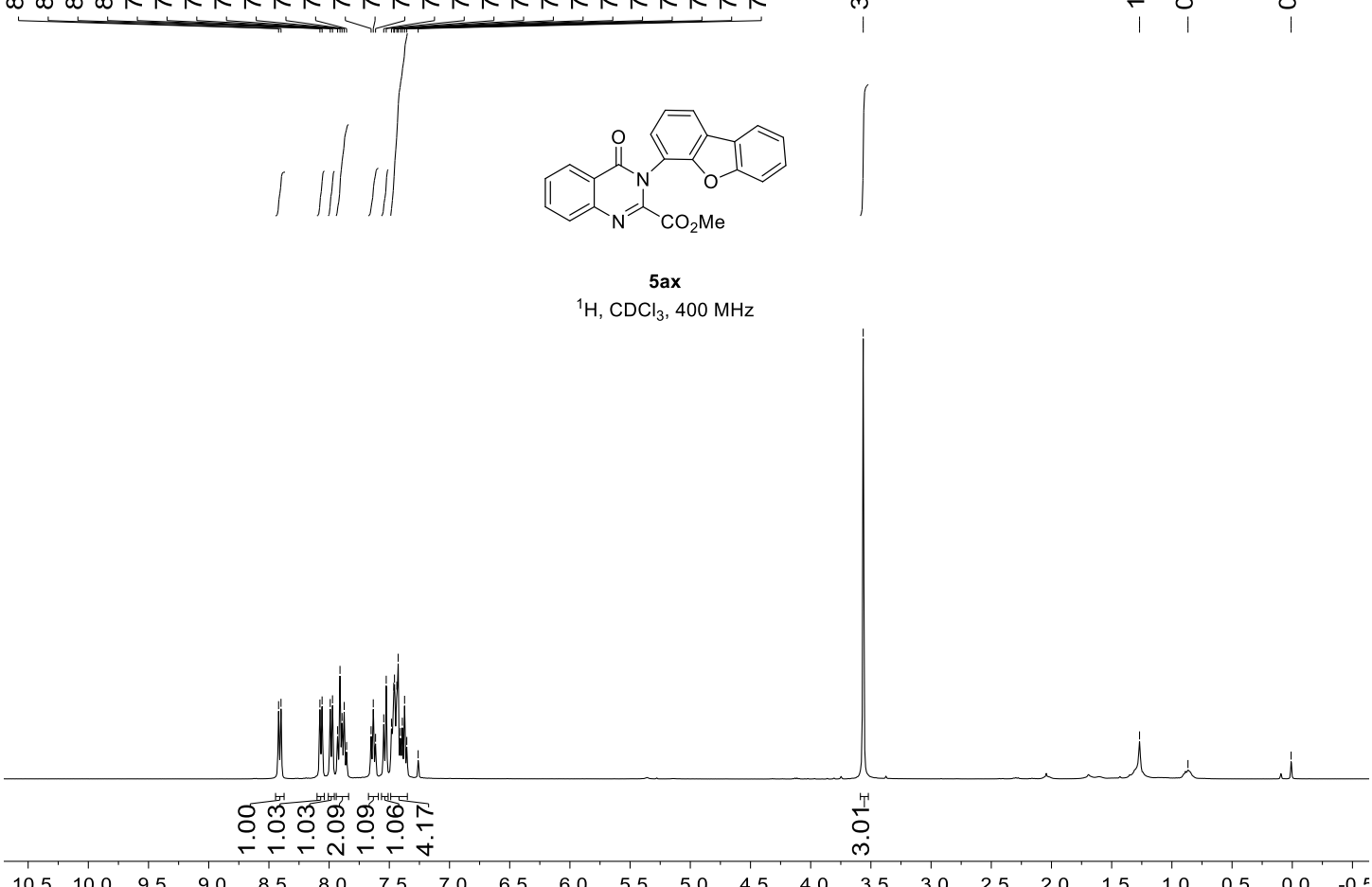

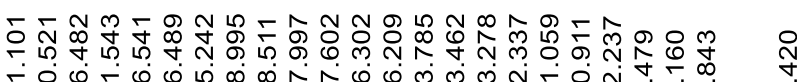

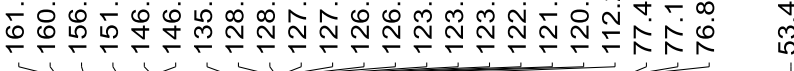

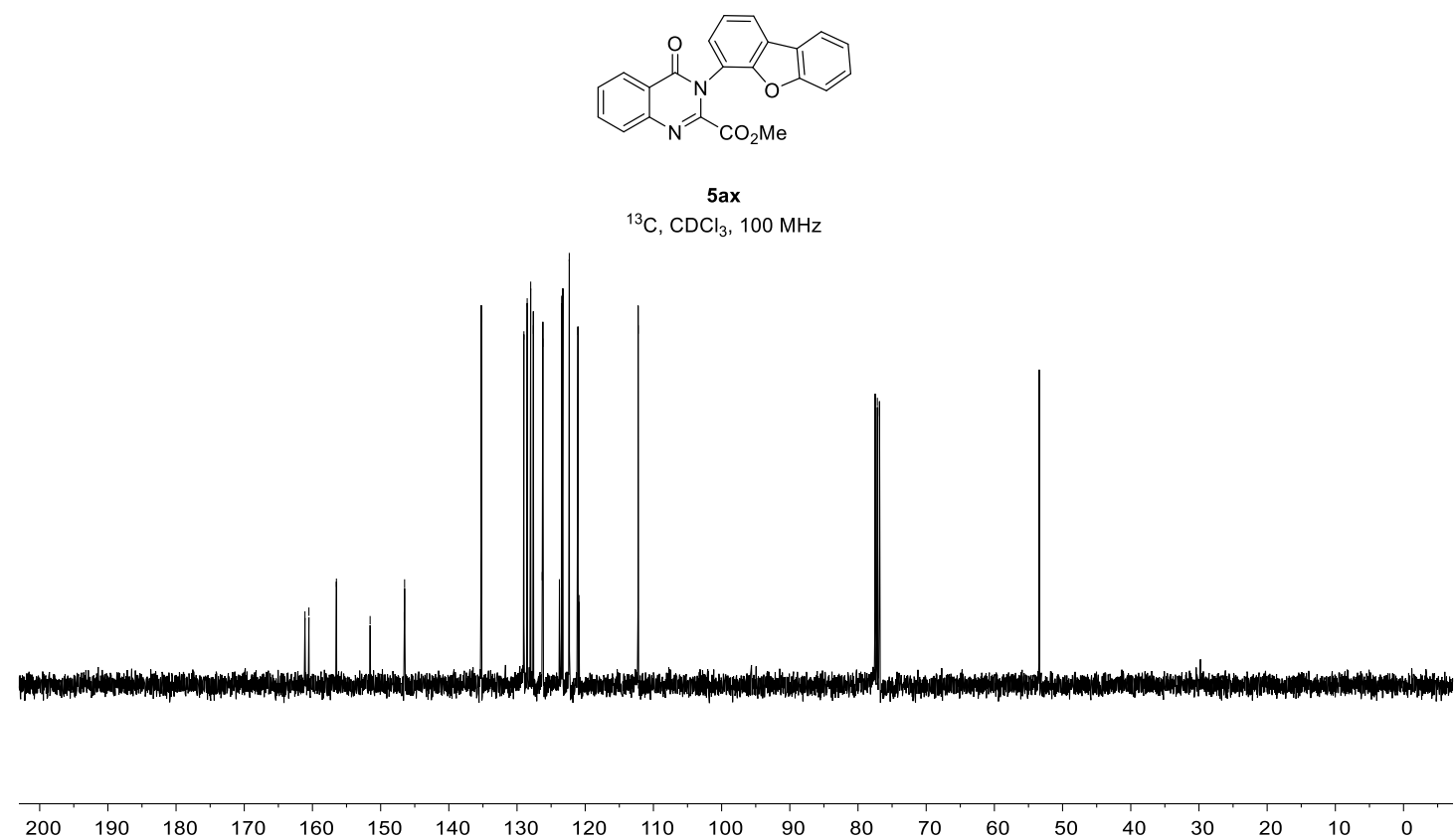




\section{\|\|$\|$}

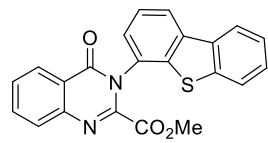

5 ay

${ }^{1} \mathrm{H}, \mathrm{CDCl}_{3}, 400 \mathrm{MHz}$

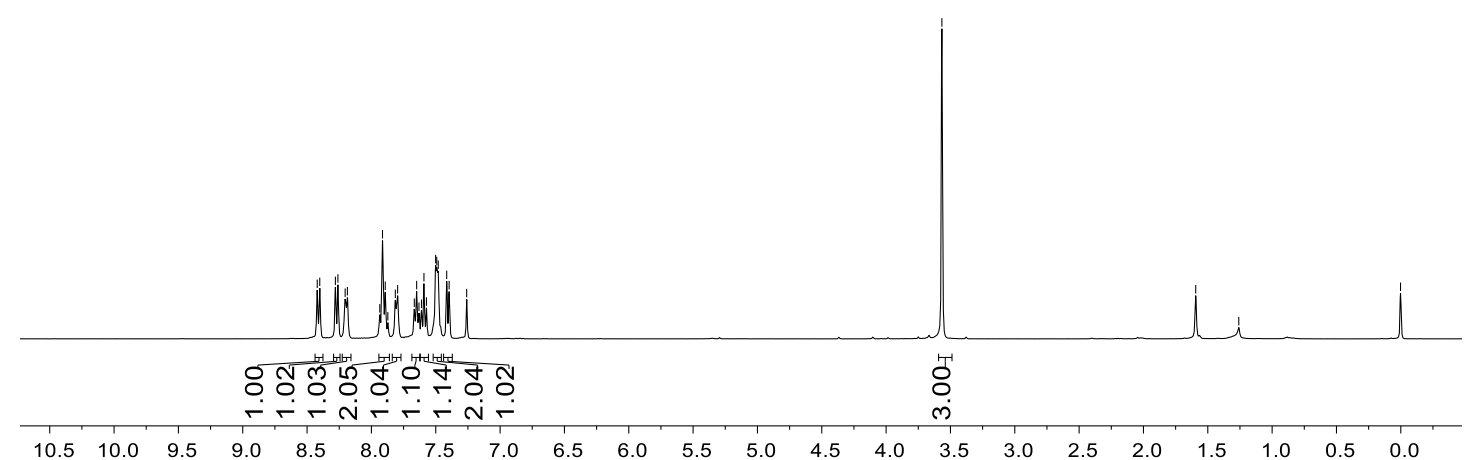

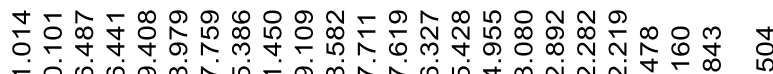

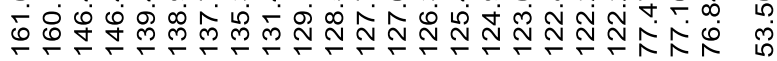

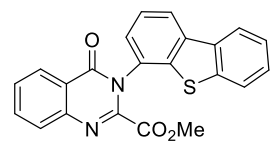

5 ay

${ }^{13} \mathrm{C}, \mathrm{CDCl}_{3}, 100 \mathrm{MHz}$

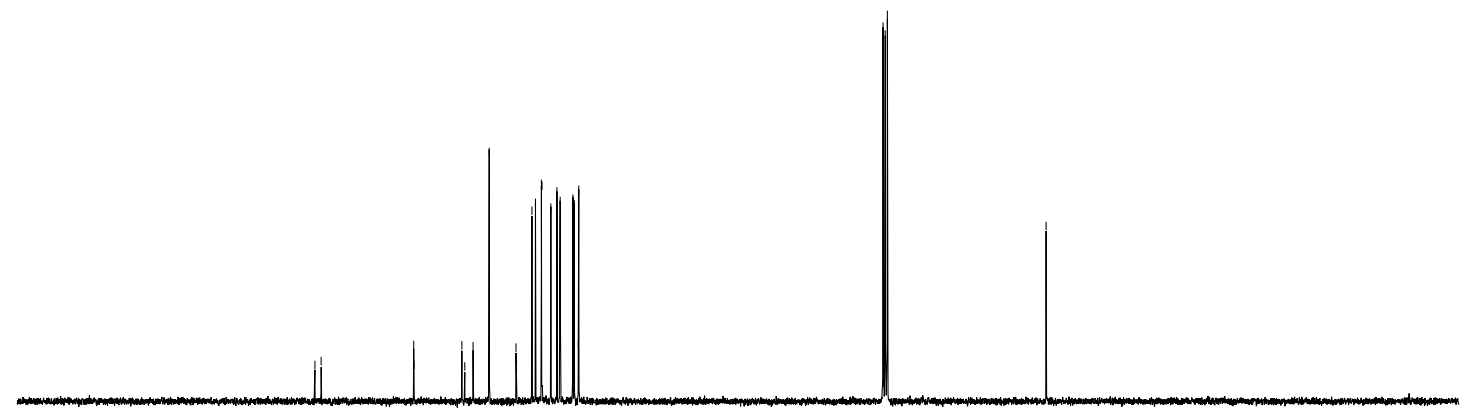

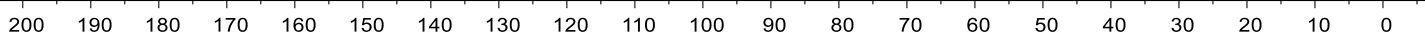




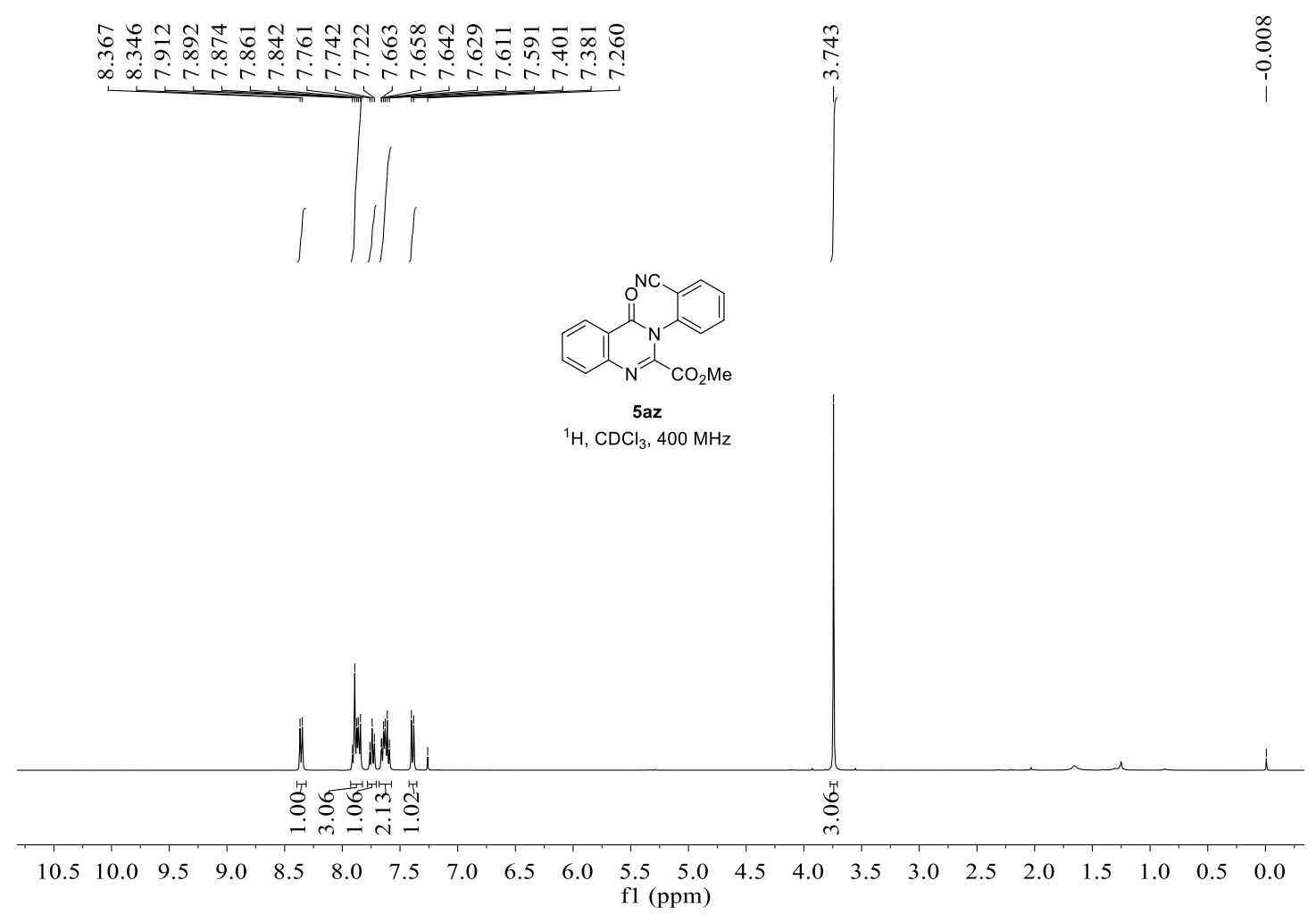

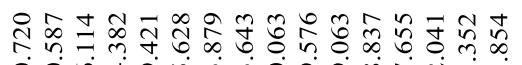

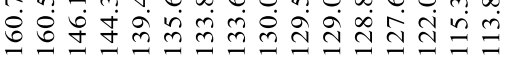

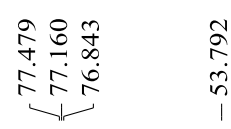

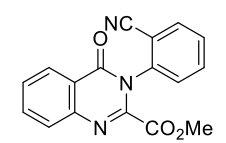

$5 a z$

${ }^{13} \mathrm{C}, \mathrm{CDCl}_{3}, 100 \mathrm{MHz}$

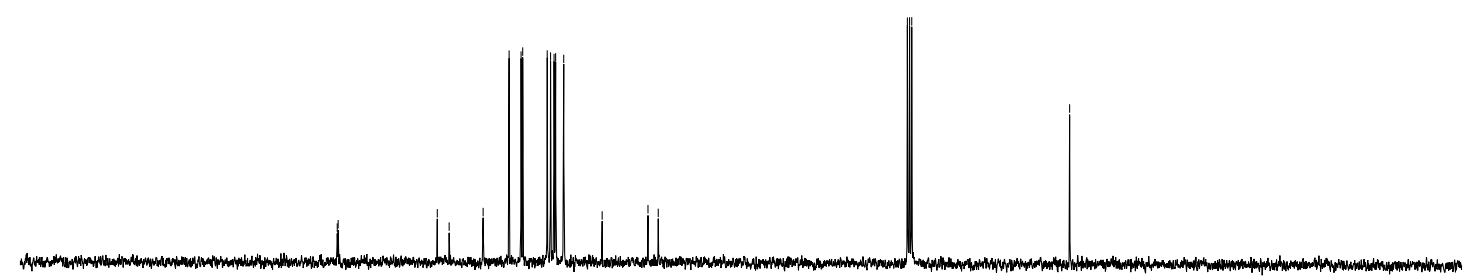

$\begin{array}{lllllllllllllllllllll}200 & 190 & 180 & 170 & 160 & 150 & 140 & 130 & 120 & 110 & 100 & 90 & 80 & 70 & 60 & 50 & 40 & 30 & 20 & 10 & 0\end{array}$ 

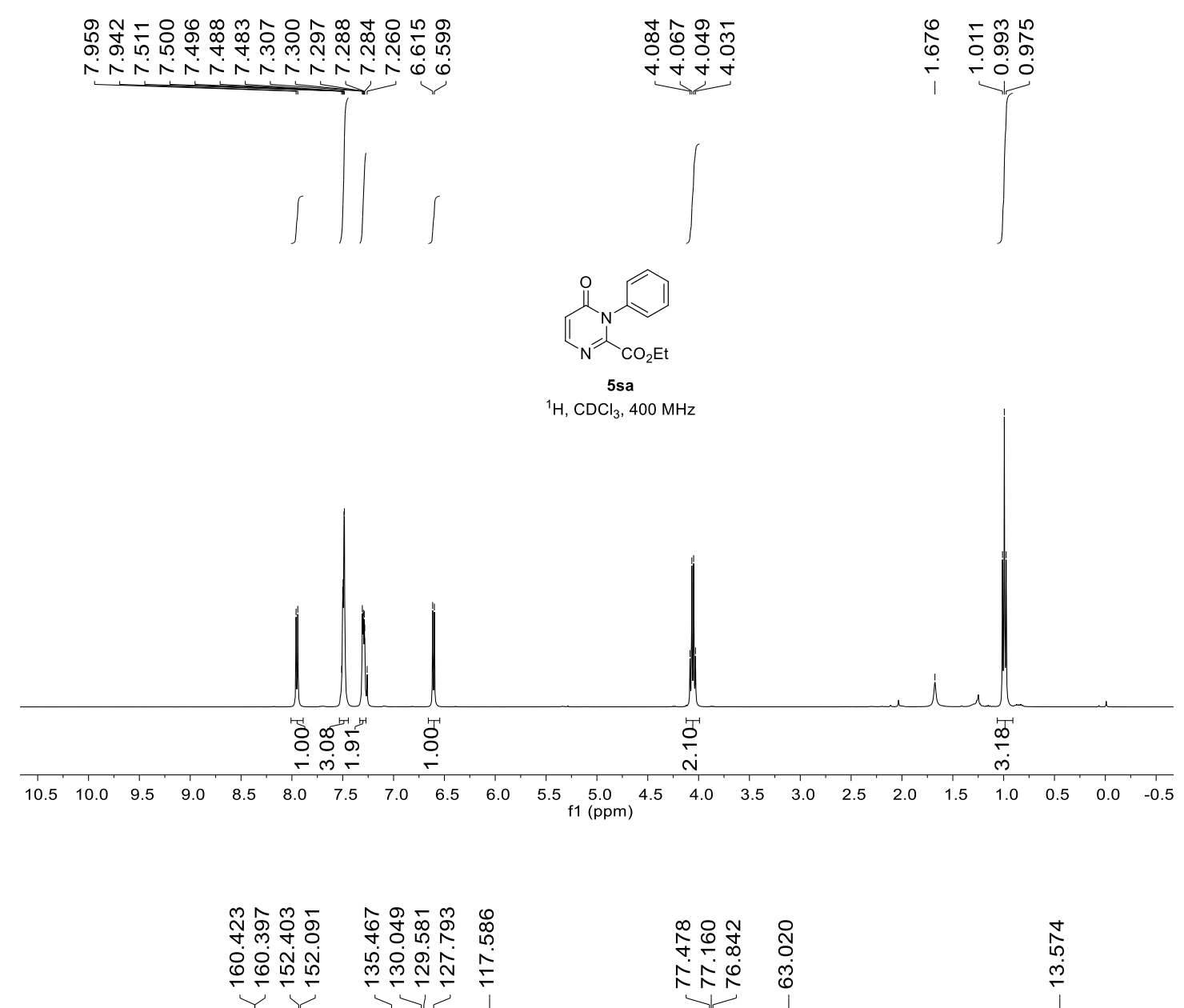

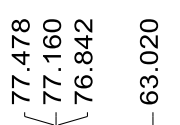

\begin{tabular}{l}
\multirow{1}{*}{} \\
$\stackrel{5}{1}$
\end{tabular}

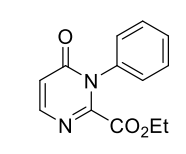

${ }^{13} \mathrm{C}, \mathrm{CDCl}_{3}, 100 \mathrm{MHz}$

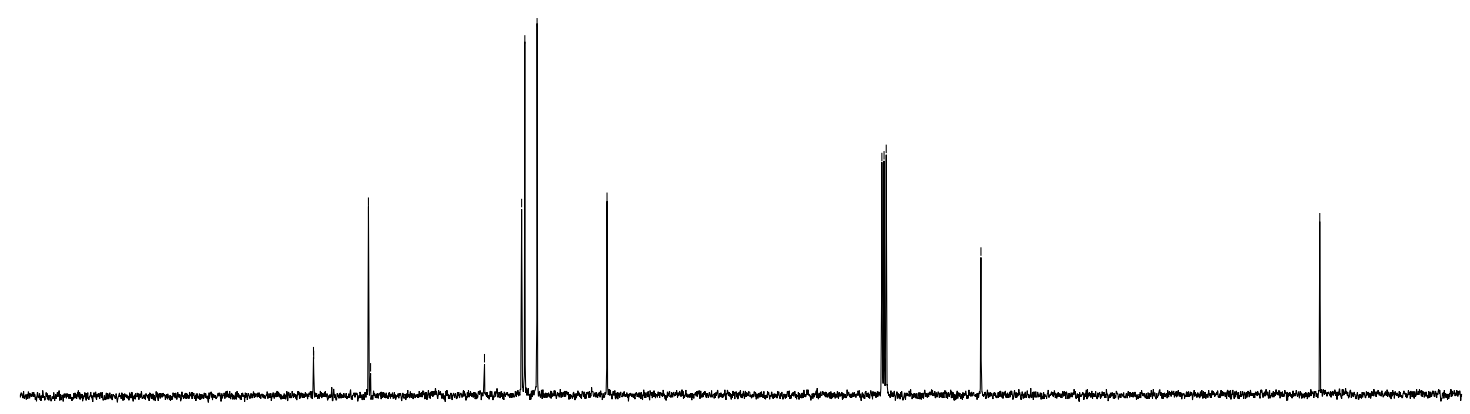

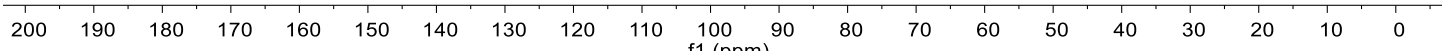



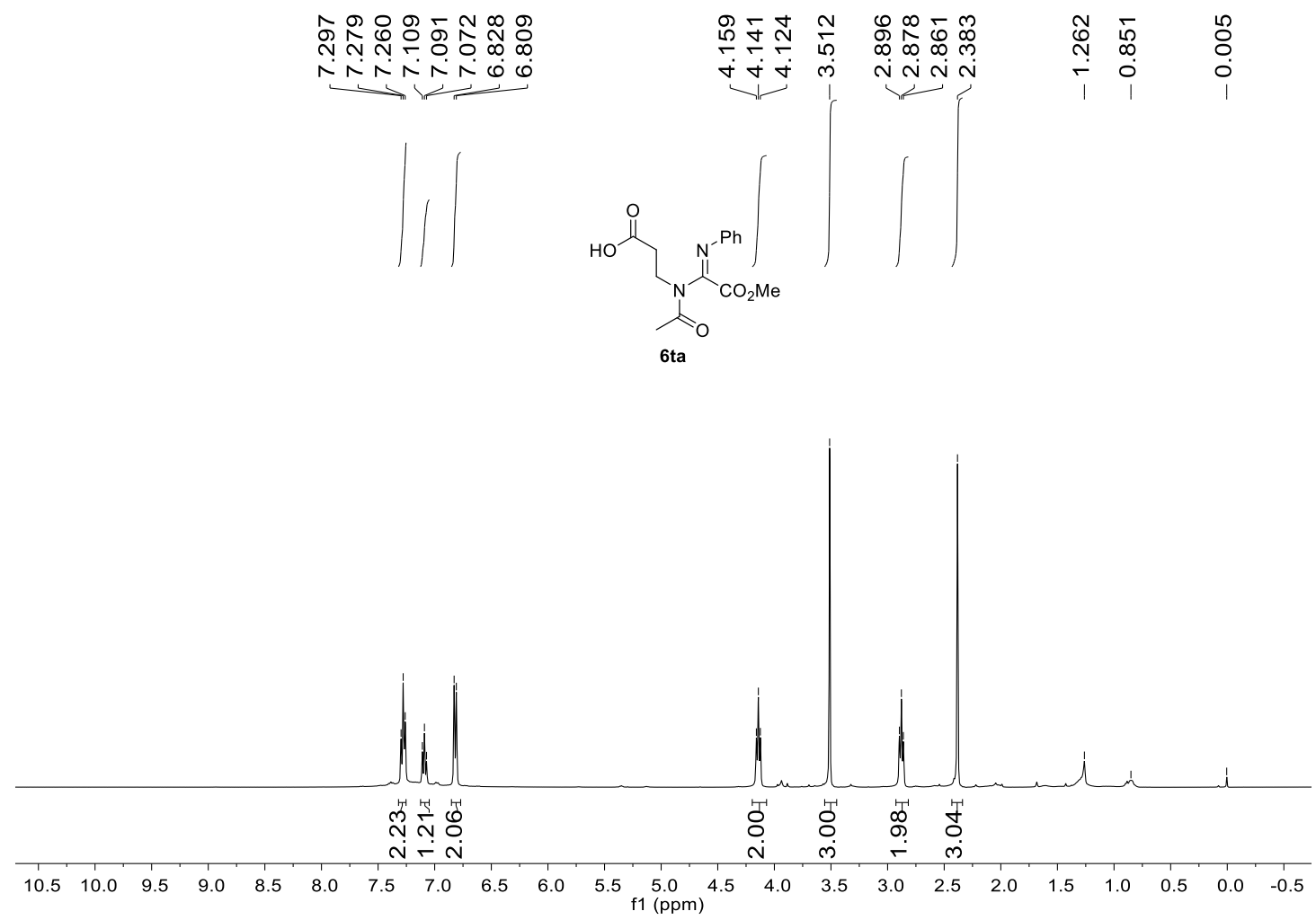

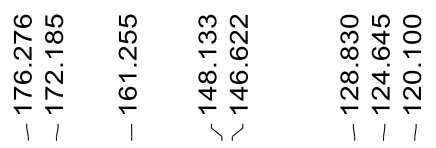

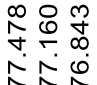

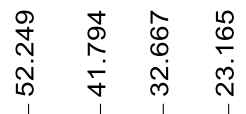

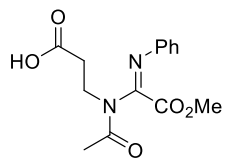

6 ta

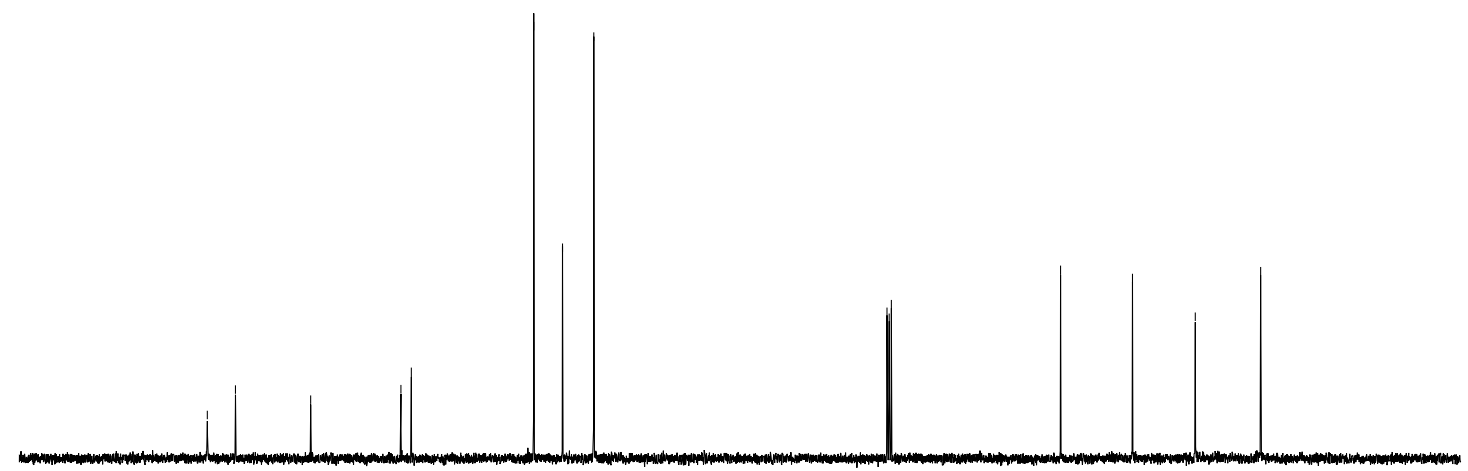

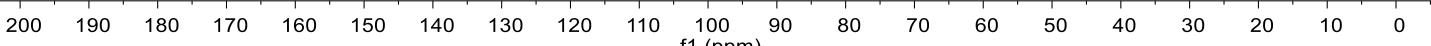




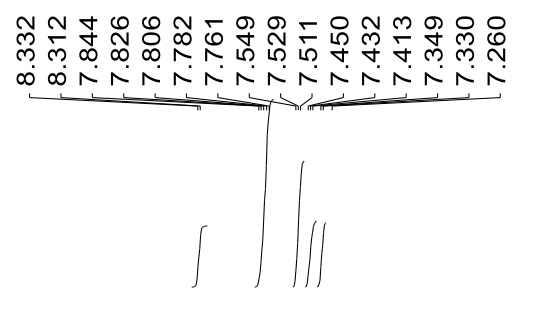

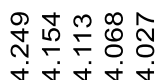

กำ

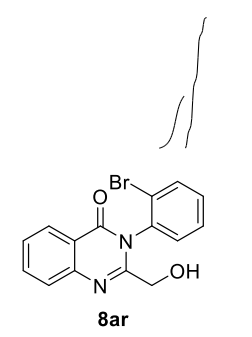

${ }^{1} \mathrm{H}, \mathrm{CDCl}_{3}, 400 \mathrm{MHz}$

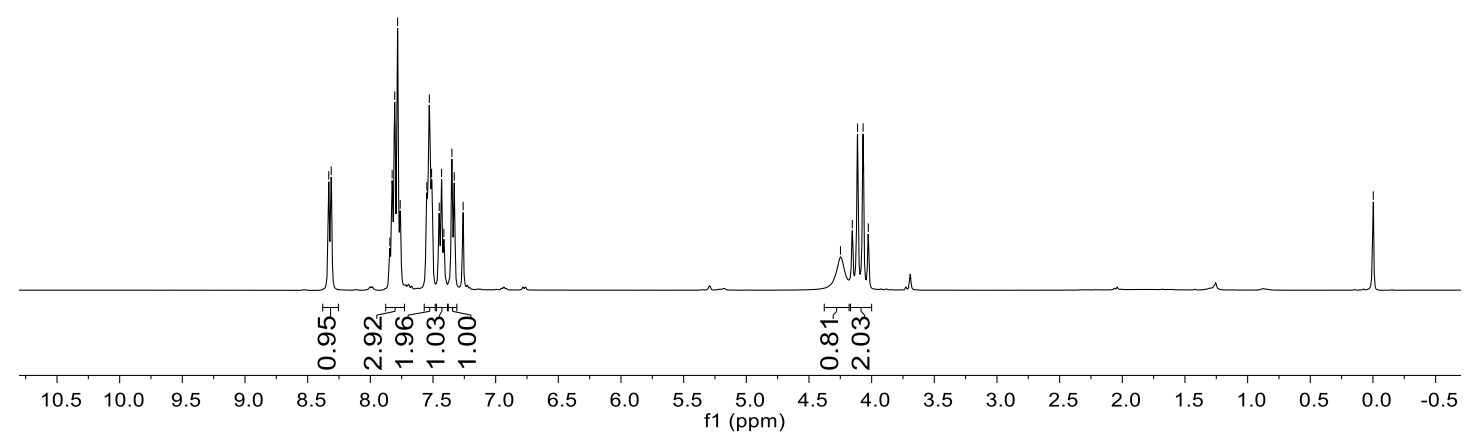

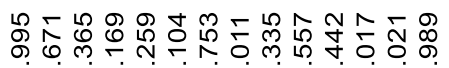

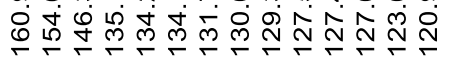

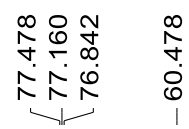
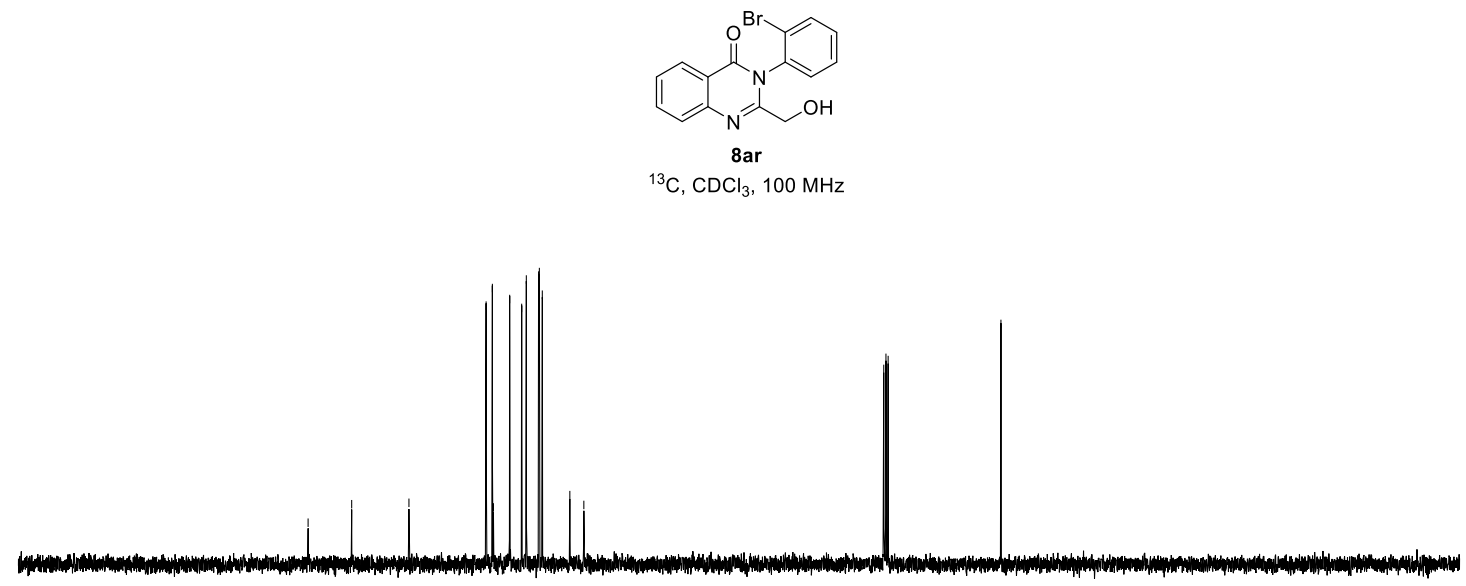

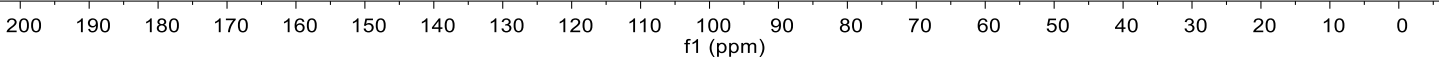



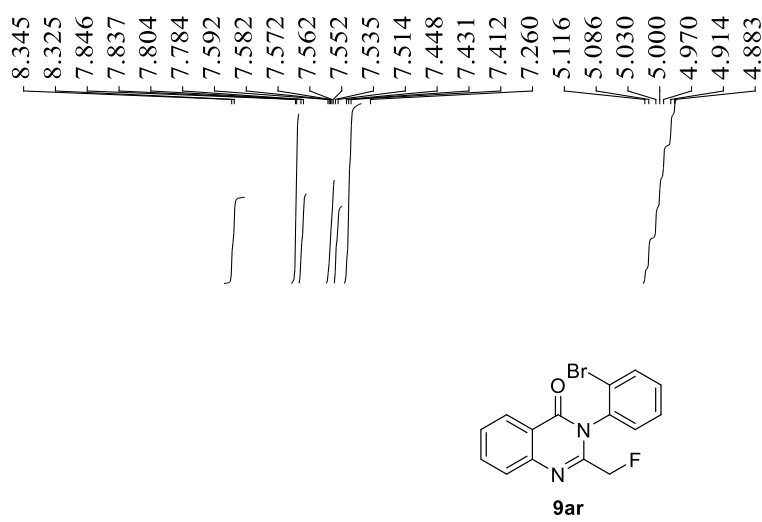

${ }^{1} \mathrm{H}, \mathrm{CDCl}_{3}, 400 \mathrm{MHz}$
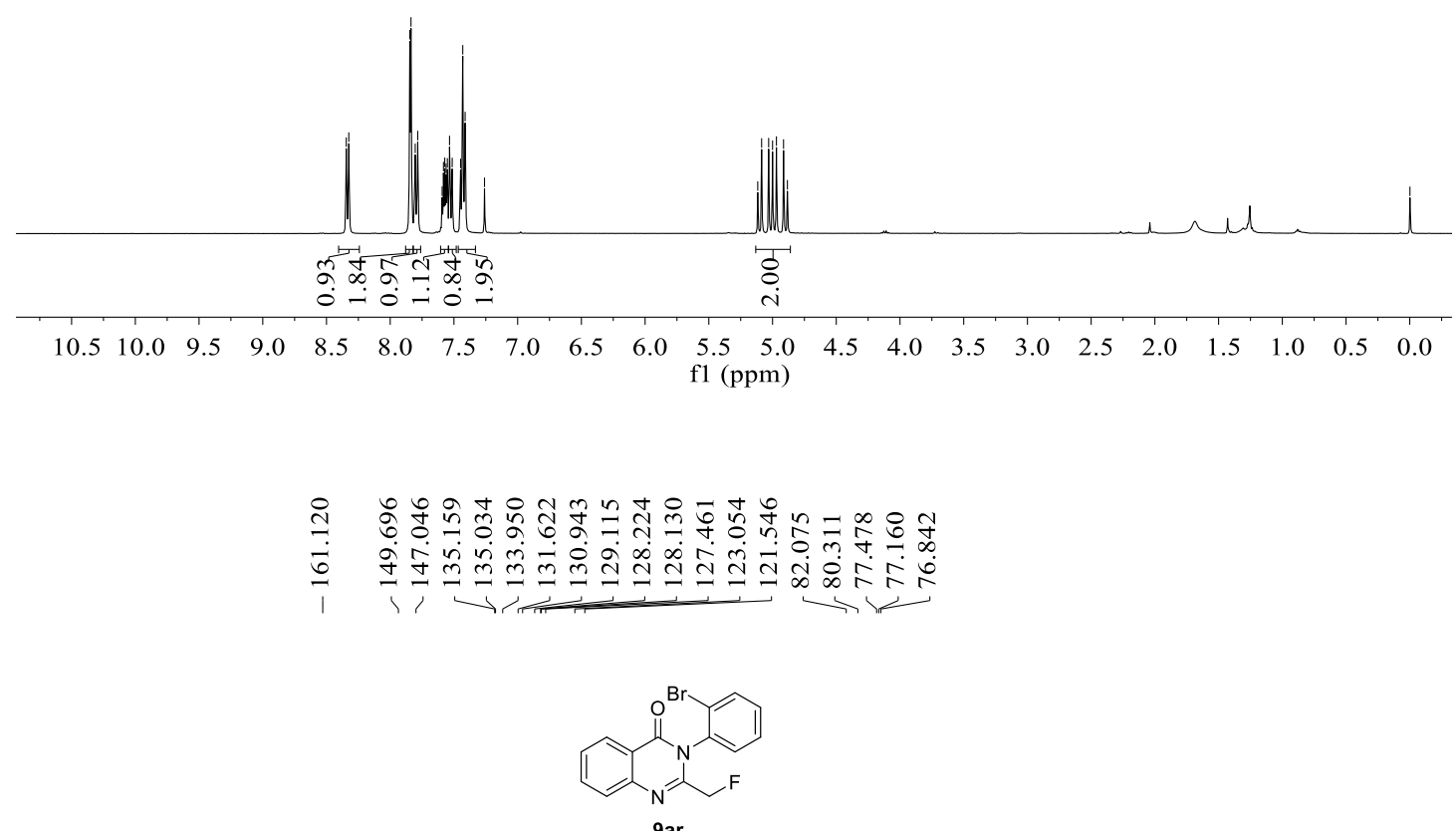

${ }^{13} \mathrm{C}, \mathrm{CDCl}_{3}, 100 \mathrm{MHz}$

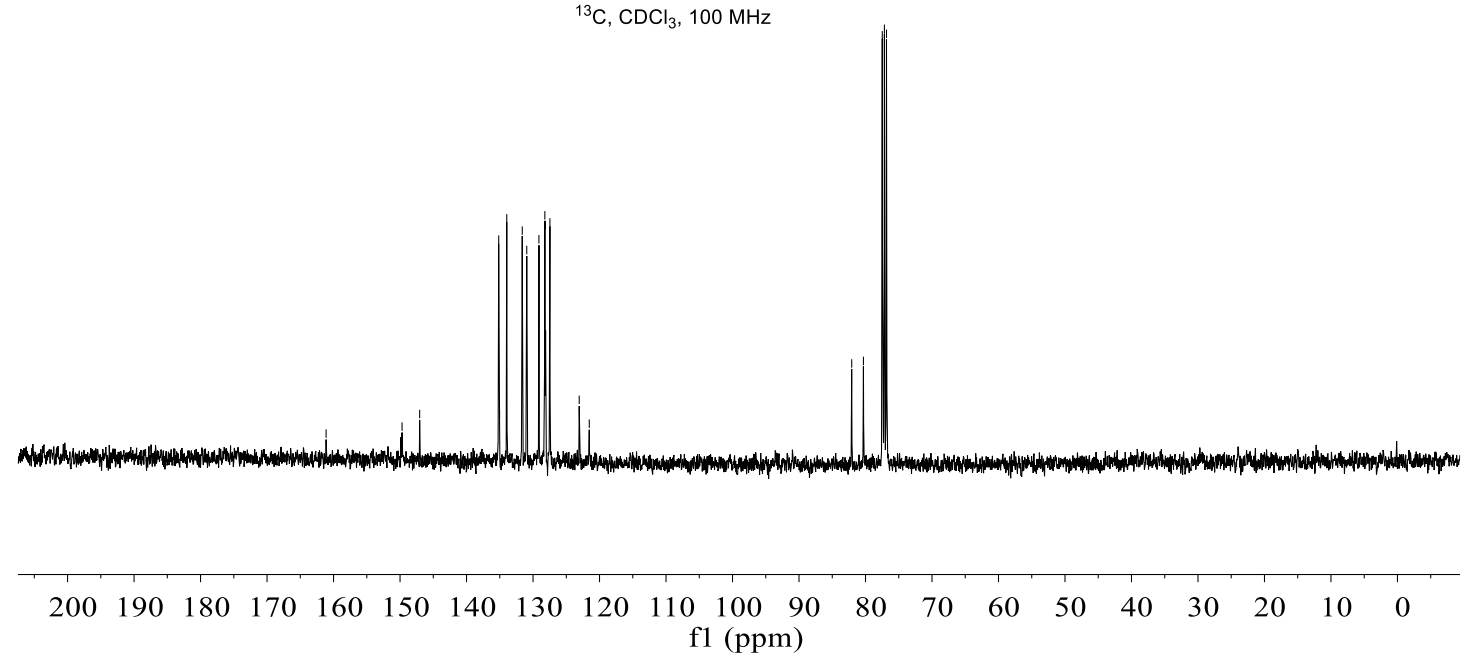




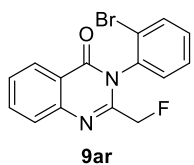

${ }^{13} \mathrm{~F}, \mathrm{CDCl}_{3}, 376 \mathrm{MHz}$

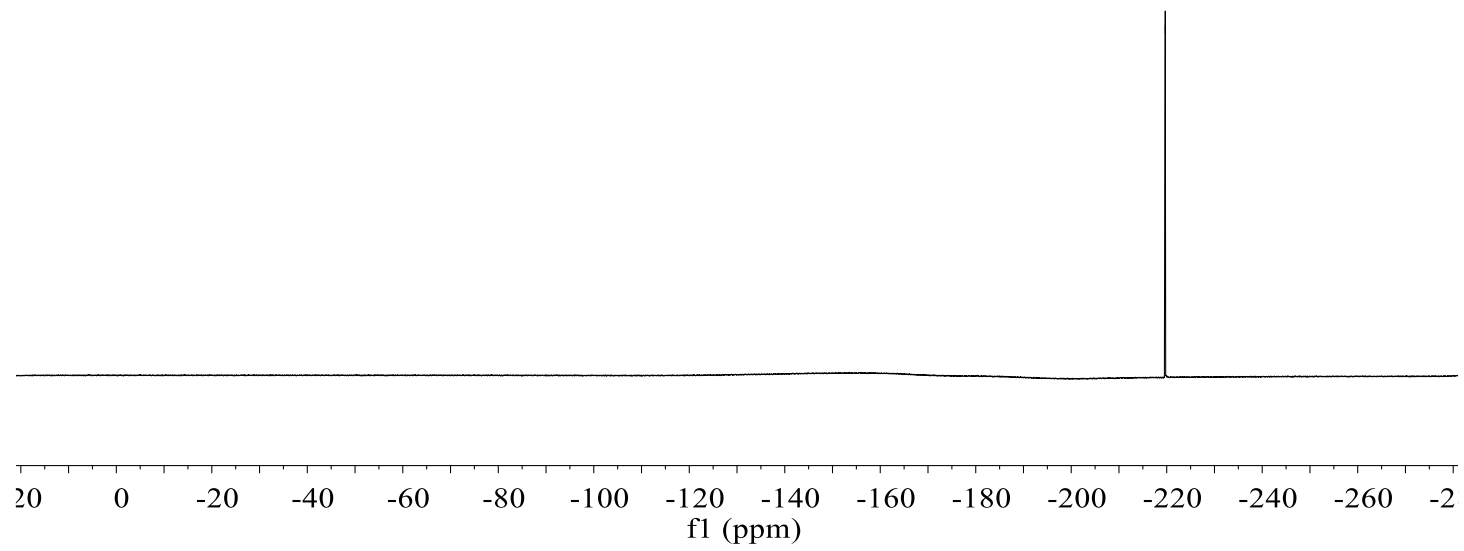




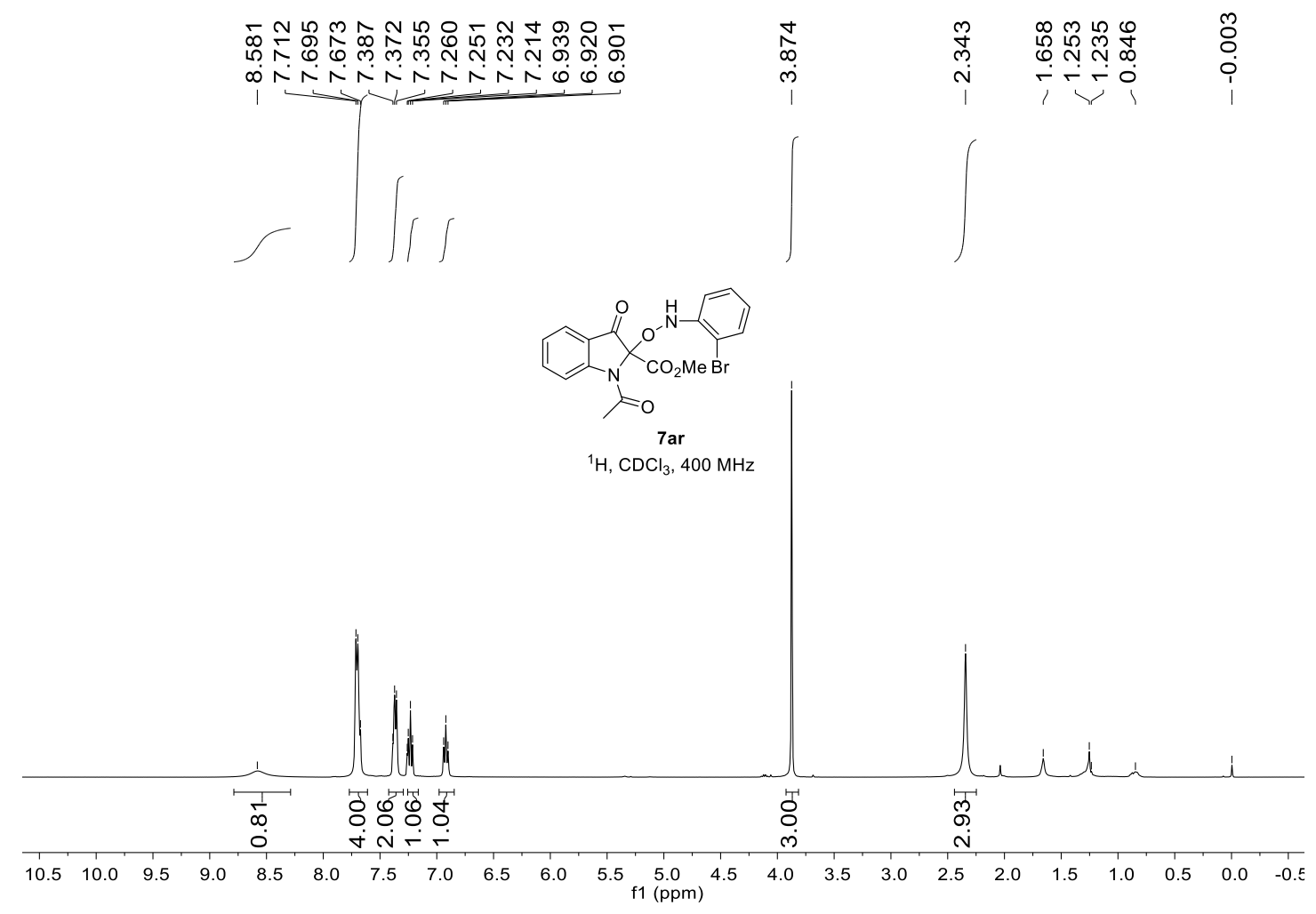

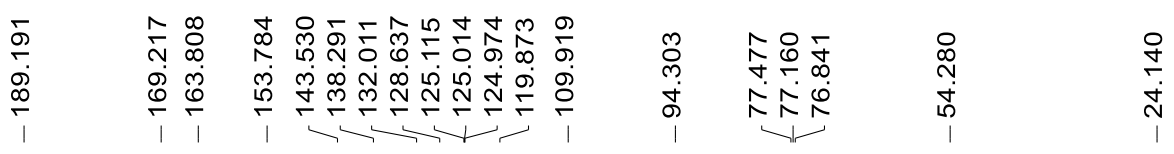

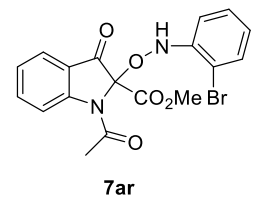

${ }^{13} \mathrm{C}, \mathrm{CDCl}_{3}, 100 \mathrm{MHz}$

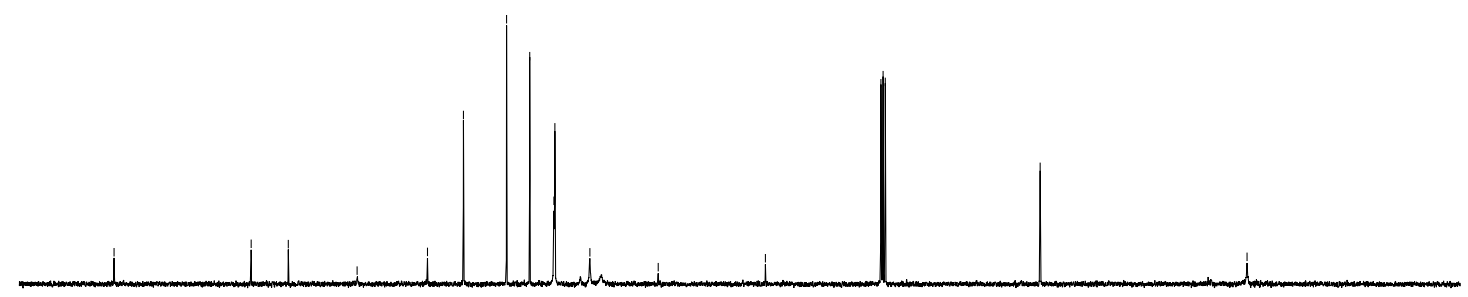

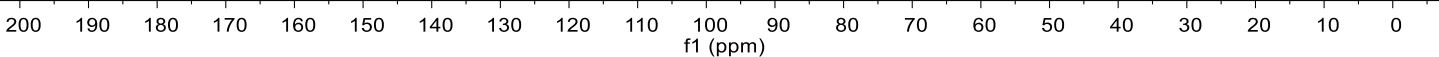




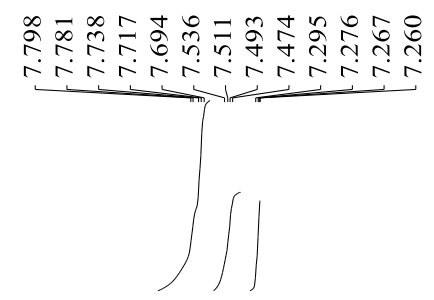

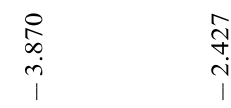

$\overrightarrow{8}$

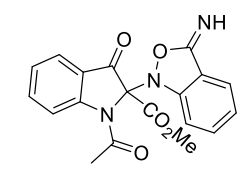

10

${ }^{1} \mathrm{H}, \mathrm{CDCl}_{3}, 400 \mathrm{MHz}$

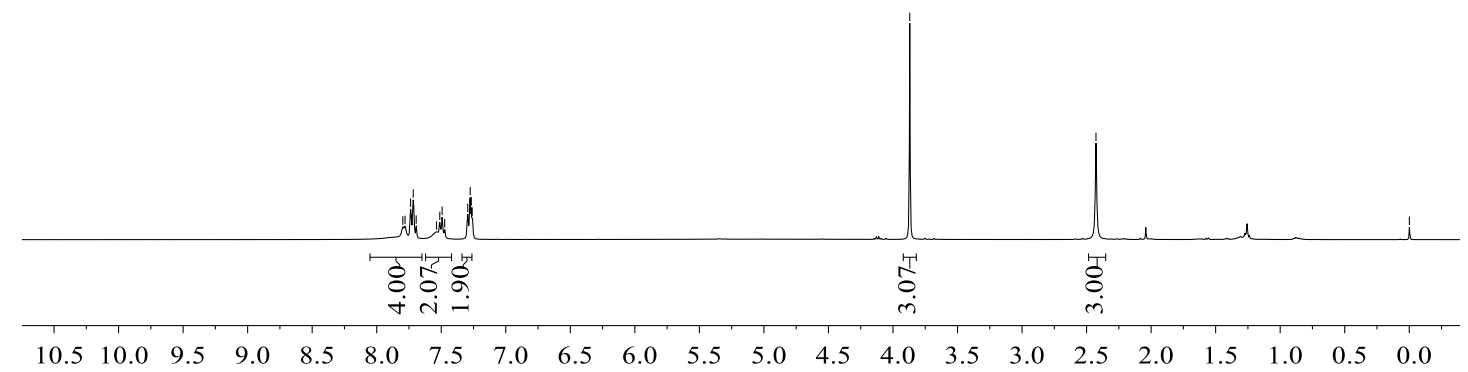

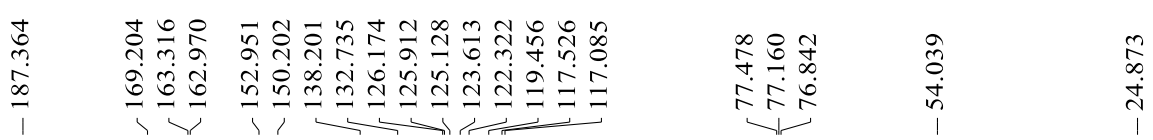

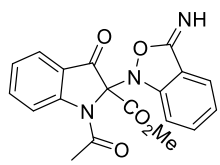

10

${ }^{13} \mathrm{C}, \mathrm{CDCl}_{3}, 100 \mathrm{MHz}$

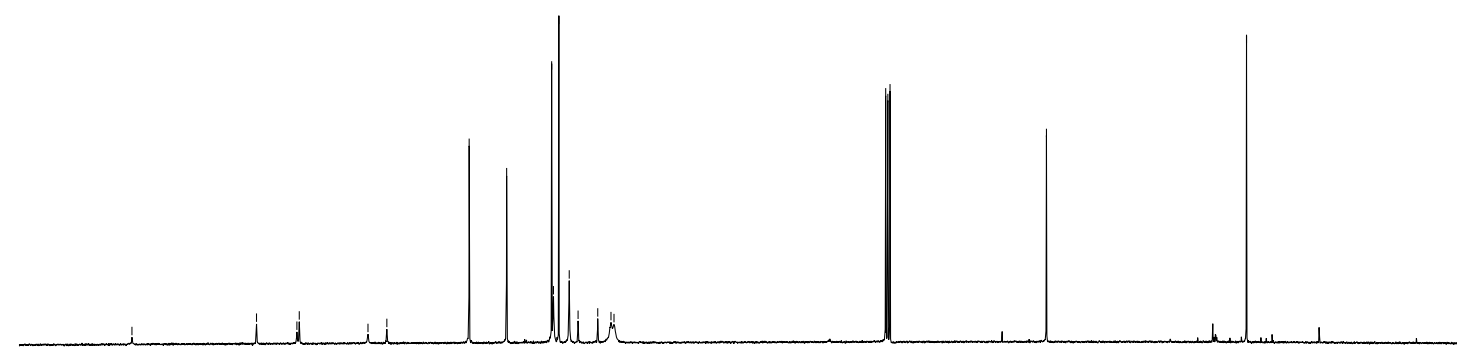

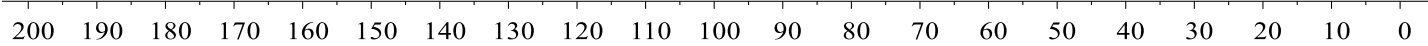




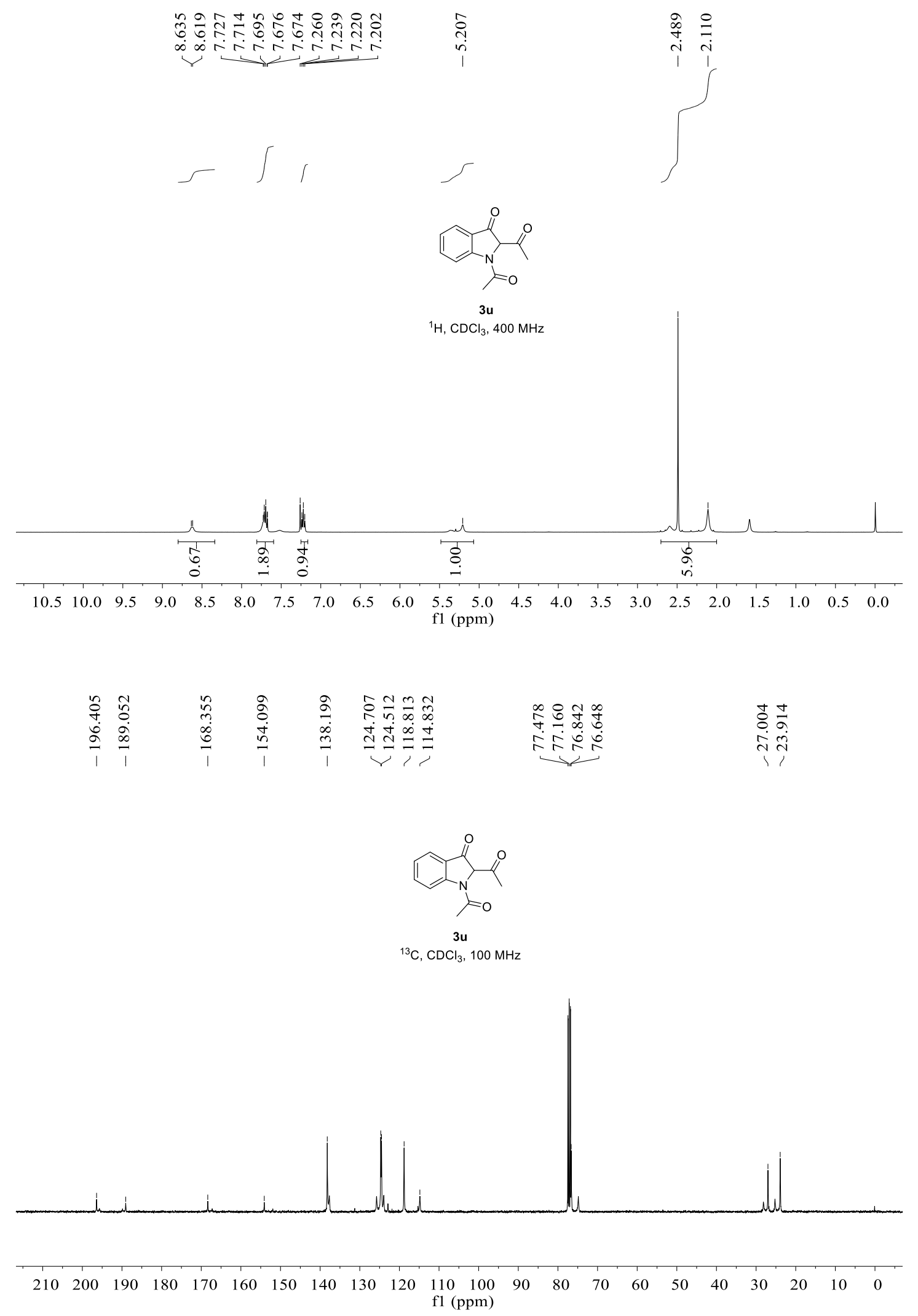




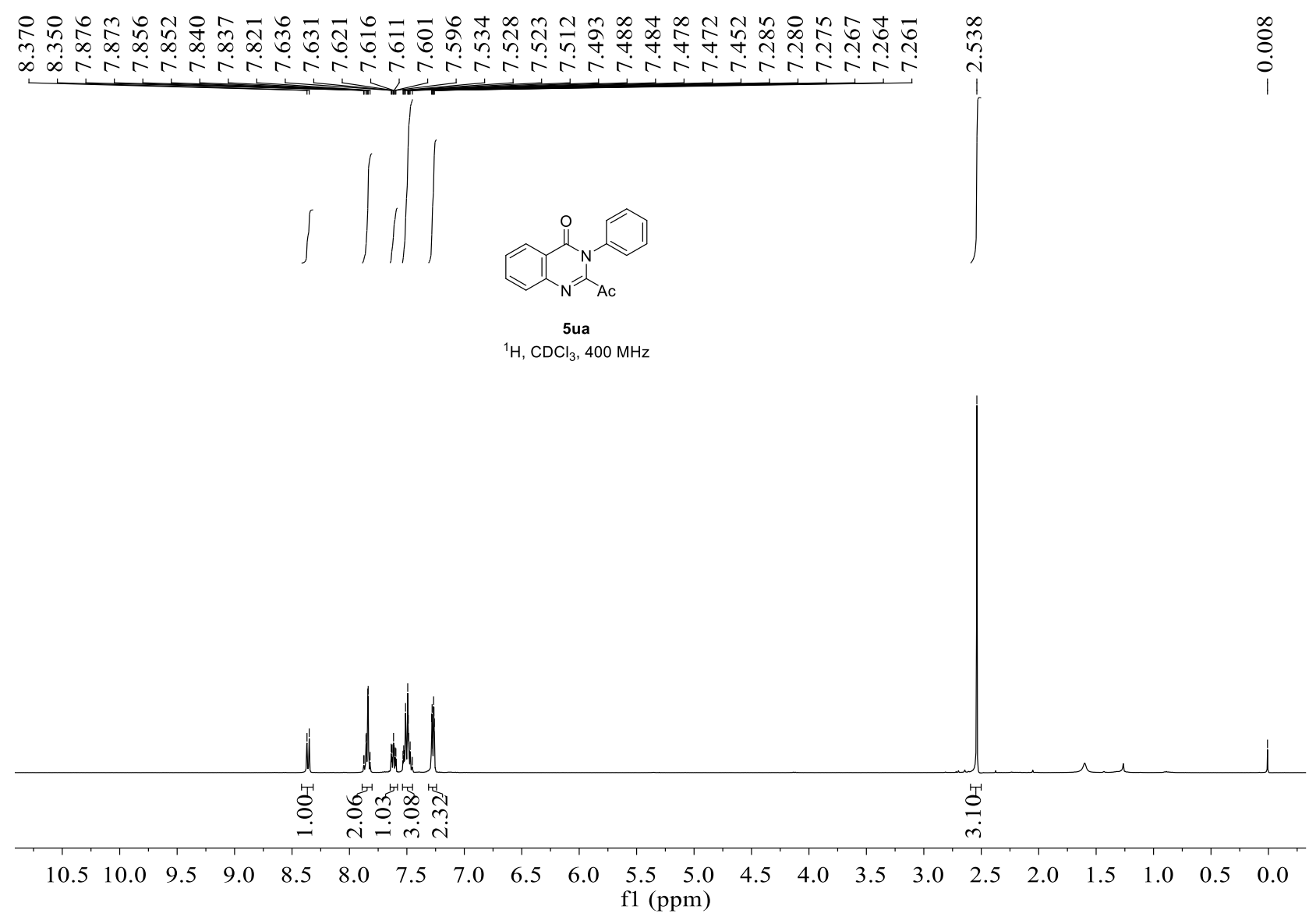

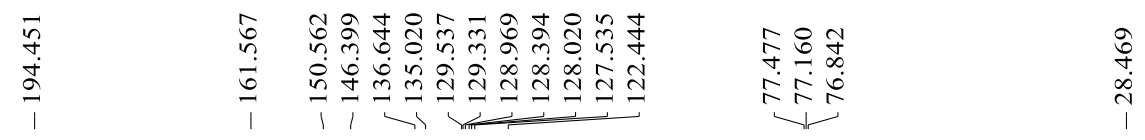

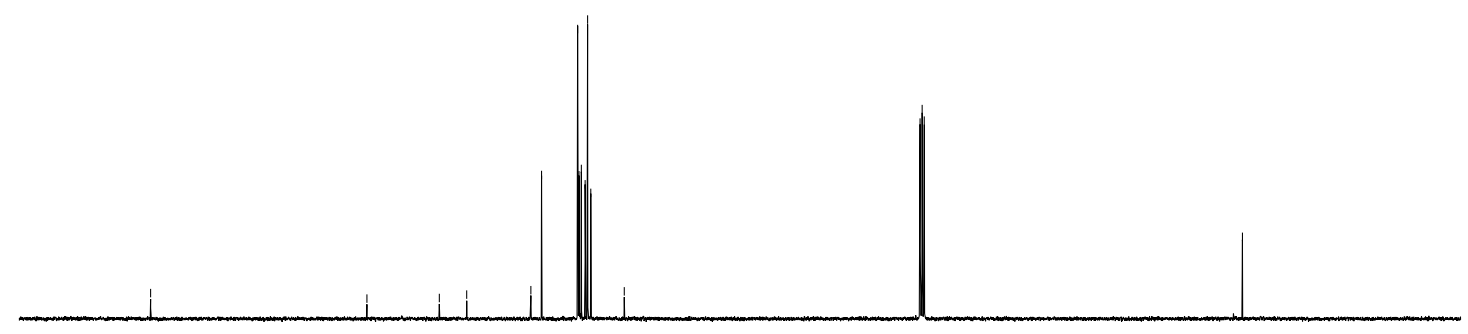

$\begin{array}{llllllllllllllllllllll}210 & 200 & 190 & 180 & 170 & 160 & 150 & 140 & 130 & 120 & 110 & 100 & 90 & 80 & 70 & 60 & 50 & 40 & 30 & 20 & 10 & 0\end{array}$

fl (ppm) 

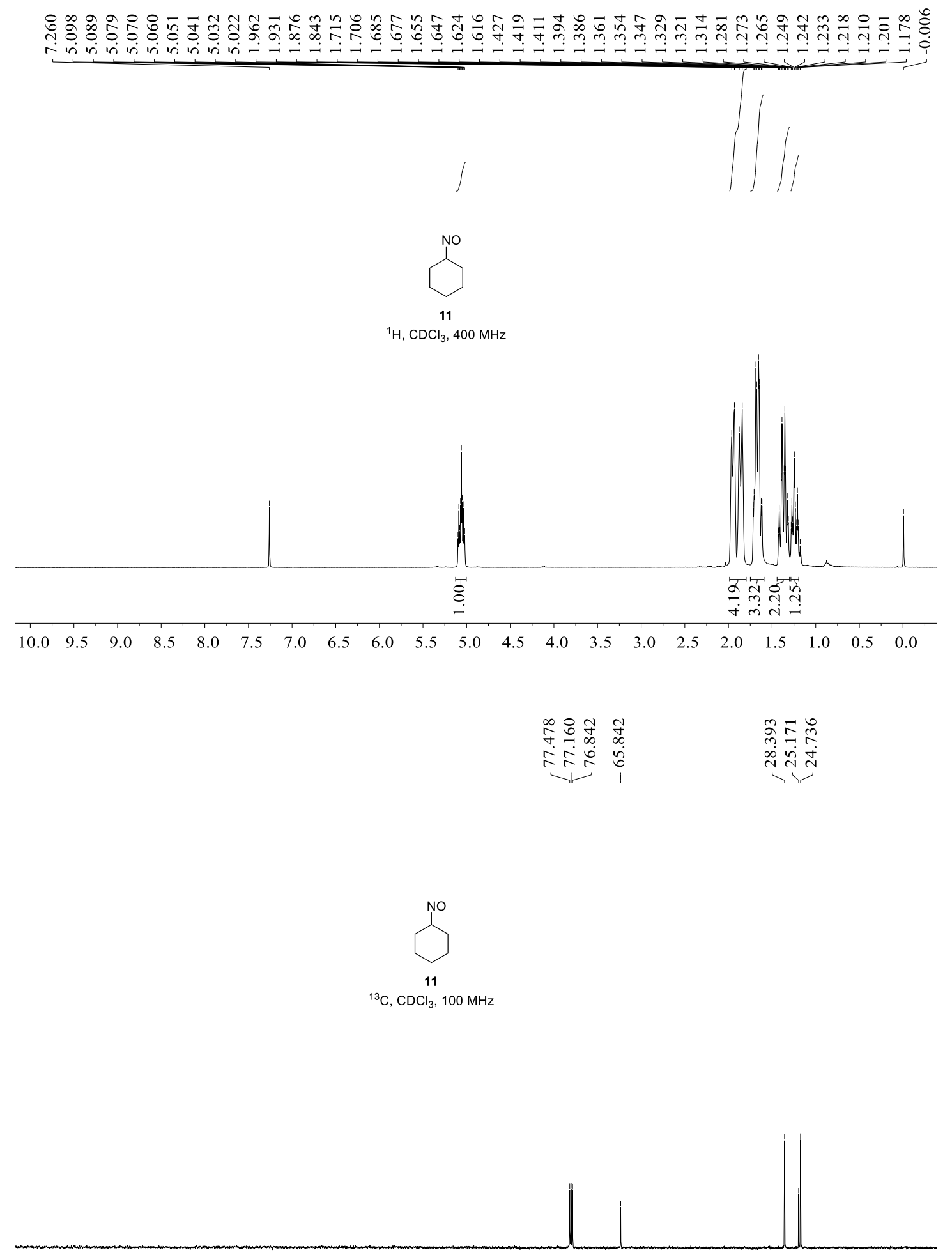

$\begin{array}{lllllllllllllllllllll}200 & 190 & 180 & 170 & 160 & 150 & 140 & 130 & 120 & 110 & 100 & 90 & 80 & 70 & 60 & 50 & 40 & 30 & 20 & 10 & 0\end{array}$ 